\title{
Layer Perfection in Ultrathin InAs Quantum Wells in $\operatorname{GaAs}(001)$
}

by

\author{
James Anil Gupta \\ B.Sc., Queen`s University, 1994 \\ M.Sc.. Queen's Iniversity. 1996
}

A THESIS SUBMITTED IN PARTIAL FULFILLMENT

OF THE REQUIREMENTS FOR THE DEGREE OF

Doctor of PhILOSOPHY

in the Department

of

Physics

(C) James Anil Gupta 1999

SIMON FRASER UNIVERSITY

August 1999

All rights reserved. This work may not be reproduced in whole or in part, by photocopy or other means, without the permission of the author. 
National Library

of Canada

Acquisitions and

Bibliographic Services

395 Wellington Street

Otiawa ON K1A ONA

Canada
Bibliothèque nationale

du Canada

Acquisitions ef

services bibliographiques

395, rue Wellington

Onawa ON KIA ONA

Caneda
The author has granted a nonexclusive licence allowing the National Library of Canada to reproduce, loan, distribute or sell copies of this thesis in microform, paper or electronic formats.

The author retains ownership of the copyright in this thesis. Neither the thesis nor substantial extracts from it may be printed or otherwise reproduced without the author's permission.
L'auteur a accordé une licence non exclusive permettant à la Bibliothèque nationale du Canada de reproduire, prêter, distribuer ou vendre des copies de cette thèse sous la forme de microfiche/film, de reproduction sur papier ou sur format électronique.

L'auteur conserve la propriété du droit d'auteur qui protège cette thèse. $\mathrm{Ni}$ la thèse ni des extraits substantiels de celle-ci ne doivent être imprimés ou autrement reproduits sans son autorisation. 


\section{Abstract}

Single- and sub-monolayer (ML) InAs films buried in CaAs(001) were grown by metalorganic vapor phase epitaxy (MOVPE) and atomic layer epitaxy (ALE). X-Ray standing wave (XSW), high-resolution X-Ray diffraction (HRXRD). and photoluminescence (PL) measurements were used to assess the layer perfection and positions of 1 and $1 / 2$.ML InAs quantum wells within the GaAs matrix. Local structure in the $1 \mathrm{ML}$ films was evaluated using glancing-incidence X-Ray absorption fine structure (XAFS) measurements performed at the In liedge.

Growth temperature effects were studied in a series of samples produced between 400 and $600^{\circ} \mathrm{C}$. The $\mathrm{XSW}$ coherent position of the In atoms decreased with increasing temperature in the 1 ML samples. suggesting an optimal growth temperature near $550^{\circ} \mathrm{C}$. according to the agreement between the data and the macroscopic elastic theory prediction of a $15 \%$ displacement of the In atoms from the GaAs(004) planes. along the growth direction. The XSW coherent fraction indicates that approximately $80 \%$ of the In atoms in this sample reside in the ideal position, a more uniform In distribution than previously reported. Low temperature PL measurements reveal that the sharpest In-excitonic emission is obtained from the $1 \mathrm{ML}$ sample grown at $530^{\circ} \mathrm{C}$. further clarifying the conditions for producing the best films.

With decreasing growth temperature the XSW results may indicate segregation of In atoms into multiple layers. This effect is reduced at higher temperatures due to the favourable kinetic conditions created in the MOVPE environment. The most important factors are the high GaAs cap growth rate and the high As overpressure. Neither of these requirements is met by the ALE growth technique. 
In-As bond lengths determined from the XAFS data also agree with the macroscopic elastic description; the In-As bonds undergo a very small $(\sim 1.8 \%)$ compression relative to their bulk bond lengths. Issues of InAs islanding were not resolved using this technique due to the low contribution of the second shell (In-In) to the XAFS signal and the very small magnitude of the bond-length distortions.

Issues of strain and bond-length distortions were further explored using $\operatorname{In}_{r} \mathrm{Ca}_{1_{-r}} \mathrm{As}$ thin-alloy films grown coherently on $\operatorname{In} \mathrm{P}(001)$. The $\ln -\mathrm{As}$ and $\mathrm{Ga}-\mathrm{As}$ bond lengths in films of varying composition. $x$, were determined using Cia- and As-K edge XAFS measurements. Comparison with a random-cluster calculation demonstrates that the external in-plane epitaxial strain imposed by pseudomorphic growth opposes the natural bond-length distortions due to alloying. 


\section{Acknowledgments}

It has been my great pleasure. during my time at $\mathrm{SFC}$, to work with a group of marvelous people. and very exceptional scientists.

Simon Watkins's lab is an excellent facility for semiconductor research. surely one of the most well-equipped in the country. My development as a young scientist has benefitted greatly from my experience working with Simon and utilizing these apparatus.

Daryl Crozier is among the most preeminent EXAFS researchers in the world and has helped pioneer many modern experimental techniques. I cannot overemphasize how beneficial it has been to work with Daryl and to draw from his extensive experience. Daryl has been most generous with his tine and is really a great guy.

Joe Woicik held my hand through the XSW and helped impress upon me the importance of experimental fastidiousness. Given the a mari usque ad mare separation between Joe and myself, we have had a surprising amount of interactions. Joe really helped me above and beyond any reasonable expectations and we ve become good friends.

I also wish to thank Dale Harrison and Detong Jiang for their help in these experiments, as well as Ken Miyano. Joseph Pellegrino, Ingrid Pickering and Barry Karlin. Georg Soerensen started me off on alot of the equipment in Simon's lab and was especially patient in his tutelage. He was even more patient as I clumsily wrestled his windsurfer.

Among my friends and fellow graduate students. I wish to thank James. Ted. Richard Arès, Phillip Yeo and the lunch gang for being there.

Closer to home my parents-in-law, the Hunters, have been wonderfully supportive 
and encouraging in my efforts. through joyous and difficult times.

My own parents and my brother. Raj, have always believed in me and been pillars of support. I could not have made it without their encouragement.

Sarah has walked along beside me and been the sun in my Vancouver sky. Clichés aside, she has been my soulmate throughout. 


\section{Dedication}

Press on. Nothing in the world can take the place of persistence.

Talent will not: nothing is more common than unsuccessful people with talent.

Genius will not; unrewarded genius is almost a proverb.

Education alone will not: the world is full of educated derelicts.

Persistence and determination alone are omnipotent.

I remember. Pop.

\section{To my Parents.}




\section{Contents}

Approval .................... i

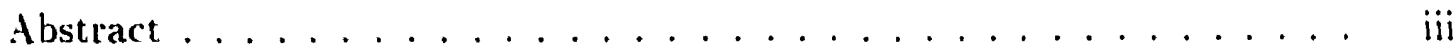

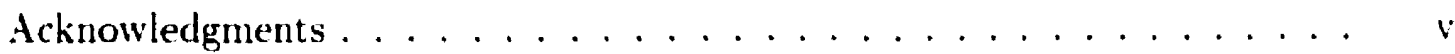

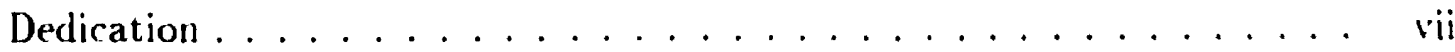

Table of Contents . . . . . . . . . . . . . . . . . viii

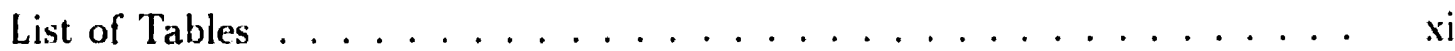

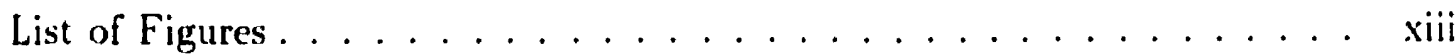

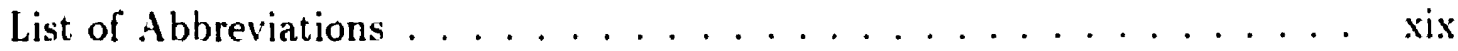

1 Introduction . ...................

$2 \quad$ Metalorganic Vapour Phase Epitaxy $\ldots \ldots \ldots \ldots \ldots \ldots$

$2.1 \quad$ Source Precursors . . . . . . . . . . . . . . . . . . i

$2.2 \quad$ Reactor Flows . . . . . . . . . . . . . . 9

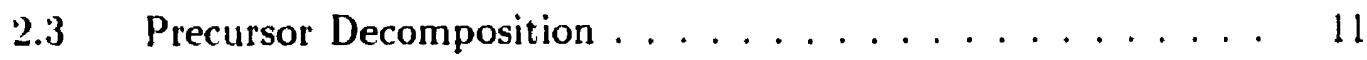

2.4 Incorporation into the Solid . . . . . . . . . . 13

$2.5 \quad$ Atomic Layer Epitaxy $\ldots \ldots \ldots \ldots \ldots$

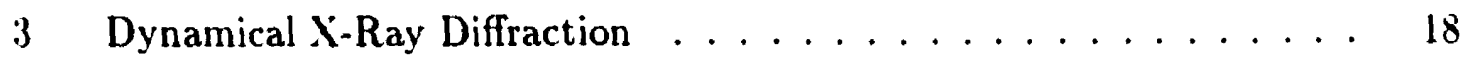

3.1 Wavefields in Crystalline Media ............ 18

$3.2 \quad$ X-Ray Standing Waves . . . . . . . . . . 26

3.2.1 Principles of the XSW Technique . . . . . . 27

$3.2 .2 \quad$ XSW Analysis $\ldots \ldots \ldots \ldots \ldots \ldots$

3.3 Simulation of X-Ray Rocking Curves . . . . . . . . . 35

4 X-Ray Absorption Fine Structure . . . . . . . . . . . . 40

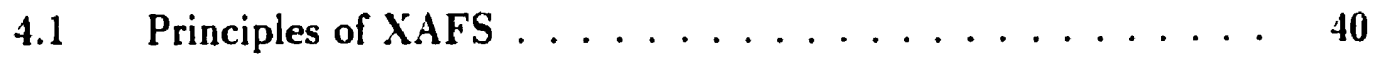


4.2 The EXAFs Expression $\ldots \ldots \ldots \ldots \ldots \ldots$

4.3 The Glancing Incidence Geometry . . . . . . . . . . . 45

4.3 .1 Parratt's Formulation . . . . . . . . 46

4.3.2 Field Amplitudes in the Crystal . . . . . . . . . 49

4.3.3 Total Reflection XSW . . . . . . . . . . . 53

$5 \quad$ Review of Literature $\ldots \ldots \ldots \ldots \ldots \ldots \ldots$

5.1 Strain in Heteroepitaxy . . . . . . . . . . . . 5i

$5.1 .1 \quad$ Macroscopic Elastic Theory . . . . . . . . . 5s

5.1 .2 Bond-Lengths in Semiconductor Alloys . . . . . . 60

5.1.3 Structural Studies of InAs SQWs . . . . . . . . 61

$5.2 \quad$ Segregation of Indium in Heteroepitaxy . . . . . . . . . 69

5.3 Reflectance Difference Spectroscopy . . . . . . . . . . . \$3

3.4 Optical Properties of Utrathin InAs Quantum Wells in GaAs 87

6 Experimental Details . . . . . . . . . . . . . . . . . 95

6.1 The MOVPE System . . . . . . . . . . . . . 9:5

$6.2 \quad$ RDS Measurements . . . . . . . . . . . . . 97

6.3 Atomic Force . Microscopy . . . . . . . . . . . . 100

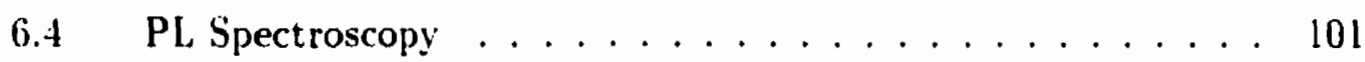

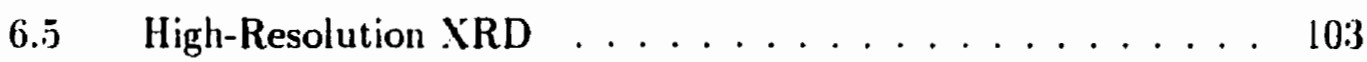

6.6 X-Ray Standing Wave Measurements . . . . . . . . . 10.5

$6.7 \quad$ Grazing Incidence XAFS . . . . . . . . . . . . 113

$i \quad$ Experimental Results . . . . . . . . . . . . . . . . 118

7.1 Analysis of Experimental XSW Data ......... 118

7.1.1 Preliminaries ............... 118

7.1.2 Standing Wave Analysis . . . . . . . . . 1.2.2

$7.2 \quad$ XSW Study of MBE-Grown Films . . . . . . . . . . 124

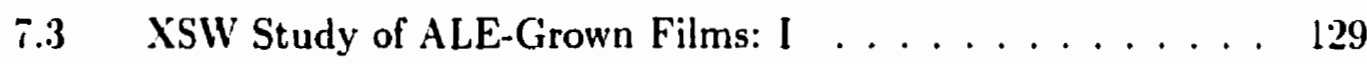

$7.4 \quad$ RDS Study of ALE Heteroepitaxy . . . . . . . . 135

7.5 XSW Study of ALE-Grown Films: II . . . . . . . . . . 143

7.6 InAs QWs in $\operatorname{GaAs}(001)$ Grown by MOVPE . . . . . . 145

i.6.1 MOVPE Growth and In-situ Characterization . . . 145 
7.6.2 XSW Study of MOVPE-Grown Films . . . . . . 155

7.6.3 X-Ray Diffraction . . . . . . . . . . 15i

i.6.4 XSW Analysis $\ldots \ldots \ldots \ldots \ldots$

7. $\quad$ XAFS Study . . . . . . . . . . . . . 169

7.7.1 Reflectivity Measurements .......... 170

7.7.2 XAFS Analysis: Preliminaries . . . . . . . . 170

7.7.3 EXAFS Analysis: Curve Fitting . . . . . . . . 172

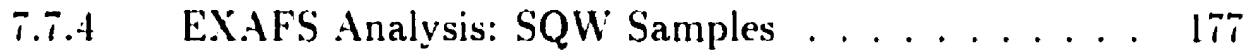

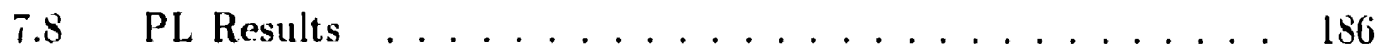

7.9 Bond-length Distortions in InGaAs/InP(001) Heterostructures 190

8 Conclusions . . . . . . . . . . . . . . . . . . . . . 205

Appendices

A Calculation of X-Ray Structure Factors . . . . . . . . . . . 209

A.1 Zincblende Structure . . . . . . . . . . . . . . 209

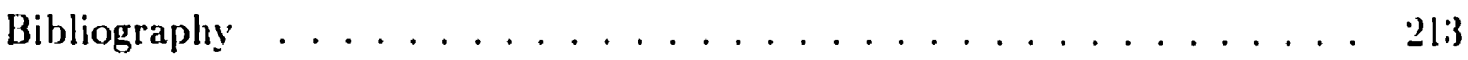




\section{List of Tables}

2.1 Material parameters for metalorganic precursors. . . . . . . . . s

2.2 Decomposition profiles of hydride and tertiarybutyl group $V$ sources. $\quad$ 13

5.1 Elastic constants of bulk compound semiconductors. . . . . . . . 60

5.2 Experimental PL energies and linewidths reported for InAs/Cia.ts het-

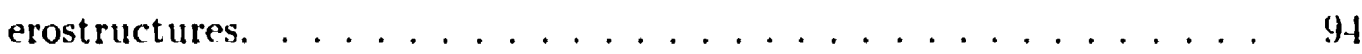

6.1 Summary of ALE and MOVPE growth conditions. . . . . . . . 9T

$6.2 \quad \mathrm{X}$-Ray emission energies. . . . . . . . . . . . . . . 109

$6.3 \quad \mathrm{X}$-Ray emission energies. . . . . . . . . . . . . 113

6.4 Ranges used for acquisition of experimental XAFS spectra. . . . . . 117

7.1 XSW results reported elsewhere for MBE-grown InAs and $\ln _{r} \mathrm{Ga}_{1-r} \mathrm{As}$ films in $\operatorname{CaAs}(001) \ldots \ldots \ldots \ldots$. . . . . . . . . . . . . . . . . . . .

7.2 $\mathrm{XSW}$ results and the In concentration determined from experimentally measured values of $P^{H}$ for MBE-grown InAs and $\operatorname{In}_{x} \mathrm{Ca}_{1-x}$ As films in $\operatorname{GaAs}(001)$.

7.3 TMGa pulse times required for $1 \mathrm{ML} /$ cycle ALE growth at different temperatures. . .................... 137

7.4 MOVPE growth rates used in SL sample preparation for in-situ RDS

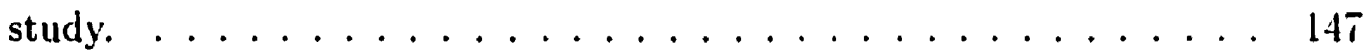

7.5 Temperature dependence of GaAs growth rate. from the GaAs cap thicknesses determined by XRD. . . . . . . . . . . 16i 
7.6 The most important paths in the InAs SQW FEFF calculation for the parallel geometry. . . . . . . . . . . . . 180

7.7 The most important paths in the InAs SQW FEFF calculation for the perpendicular geometry. . . . . . . . . . . . . . . 181

7.8 Fit parameters obtained from the experimental In-As first shell EXAFS.18I

7.9 Alkyl flow rates and input vapour phase composition for MOVPE growth of InGaAs layers on InP. The solid phase composition, thickness and cap thickness were determined by XRD. . . . . . . . . . 191

7.10 Experimental determination of the bond lengths in powder standards from fitting with FEFF6b models. . . . . . . . . . . . . . . 199

7.11 Experimental determination of the bond lengths and coordination numbers in $\operatorname{In}_{s} \mathrm{Ga}_{1-r}$ As alloys on $\operatorname{In} \mathrm{P}(001) \ldots \ldots \ldots \ldots$

A.1 Coefficients for analytical approximation to the atomic scattering factors for relevant elements. . . . . . . . . . . . . . . . 211 


\section{List of Figures}

1.1 A single layer of In atoms embedded in a $\operatorname{GaAs}(001)$ crystal. . . . . 2

2.1 Prepurified hydrogen gas flowing through a stainless steel bubbler containing liquid source metalorganic molecules. . . . . . . . . . s

2.2 Metalorganic precursor molecules. . . . . . . . . . . . 9

2.3 The vertical MOVPE reactor cell employed in this work. . . . . . 11

2.4 Different regimes of epitaxy according to the growth temperature. $T_{g}$. $\quad$ I.

2.5 A schematic illustration of step flow growth. . . . . . . . . 16

3.1 The Ewald sphere and Bragg's law. . . . . . . . . . . . . 19

3.2 The Ewald sphere in reciprocal space, showing the wavevector corrected by the average index of refraction. . . . . . . . . . . 2.3

3.3 Shape of the (004) symmetric Bragg reflection for a GaAs crystal. . . 25

3.4 Schematic of the XSW technique. . . . . . . . . . . . . . . 29

3.5 Reflectivity and phase of the symmetric $\mathrm{GaAs}(004)$ back-reflection and XSW intensities for different values of $H z_{H}$ within the GaAs unit cell. 32

3.6 The recursion method for dynanical diffraction simulations. . . . . . 37

3.7 Simulated X-Ray rocking curves for a $\mathrm{GaAs}(001) / 1 \mathrm{ML}$ InAs/100 A GaAs structure and for a $35 \times(1 \mathrm{ML}$ InAs/100 $A$ GaAs $)$ superlattice on $\operatorname{GaAs}(001) \ldots \ldots \ldots \ldots \ldots \ldots \ldots \ldots \ldots$

4.1 The propagation of the spherical photoelectron wave excited from an atom in the zincblende lattice. . . . . . . . . . . . . 42

$4.2 \mathrm{Ga}$ K-edge XAFS from a GaAs powder sample. . . . . . . . . . 43 
4.3 The propagation of a plane wave in a layered structure. . . . . . . 46

4.4 The reflectivity calculated for layered InAs/GaAs structures and the electric field intensity at the location of the In atoms for different GaAs cap thicknesses. . . . . . . . . . . . . . . .

4.5 The reflectivity, shown on a logarithmic scale, calculated for layered InAs/GaAs structures with different GaAs cap thicknesses. . . . . . 52

4.6 The reflectivity calculated for a GaAs crystal and the penetration depth of the X-Rays into the crystal. . . . . . . . . . . . . 5.1

4.7 The reflectivity and phase for a CiaAs crystal and the electric field intensity at the crystal surface and at twice the critical period. . . . .

5.1 Strain accommodation in heteroepitaxy. . . . . . . . . . 59

5.2 The first-neighbour environment of atoms in the zincblende lattice. . 65

5.3 The sixteen-bond InGiaAs cluster used in the model of Reference [62]. 68

5.4 Schematic representation of the energy of an In atom as a function of depth within a GaAs film. ............

5.5) An RDS spectrum of the GaAs $(001) \mathrm{d}(+4 \times 4)$ reconstruction. . . . . . 84

5.6 Models of the $\mathrm{c}(4 \times 4)$ and $(2 \times 4)$ reconstructions of $\mathrm{GaAs}(001) \ldots \ldots$. . .

5.7 Illustration of radiative transitions involving electrons bound to neutral donors $\left(D^{0}\right)$ and holes bound to neutral acceptors $\left(A^{0}\right) \ldots \ldots . . .89$

5.8 Illustration of band alignment in InAs/GaAs SQW structures. . . . . 91

6.1 A schematic of the vertical MOVPE reactor and the RDS system. . . 96

6.2 Determination of the RDS background signal. . . . . . . . . 99

6.3 A schematic of the atomic force microscope and the tip-surface inter-

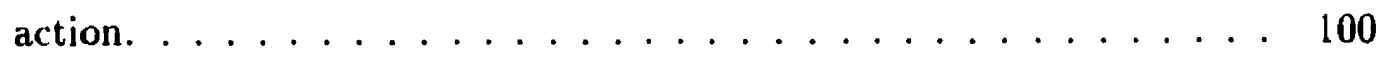

6.4 a) Liquid He cryostat used in PL measurements. b) The geometry for sample illumination and signal acquisition. . . . . . . . . 102

6.5 The BEDE $\mathrm{D}^{3}$ system. . . . . . . . . . . . . . . . 104

6.6 A schematic of the experimental back-reflection XSW arrangement at beamline X24A at the NSLS. . . . . . . . . . . 107

6.7 The experimental chamber used in XSW measurements . . . . . 108 
6.8 The Ge fluorescence detector and instrumentation. . . . . . . . . 110

6.9 The transmission of X-Rays through a $50.8 \mu \mathrm{m}$ Be window. . . . . . 111

6.10 The SSRL GIXAS stage. . . . . . . . . . . . . . . . . 114

6.11 The transmission of $\mathrm{X}$-rays through a $1.5 \mathrm{~mm}$ Al filter $\ldots \ldots \ldots 11.5$

6.12 The experimental GIXAS geometry. . . . . . . . . . . . 116

7.1 Bragg reflectivity and XSW intensity measured as a function of photon energy for a reference GaAs crystal. . . . . . . . . . . . . . . . 119

7.2 Photon energy dependence of the reflectivity and the In-L FLY near the GaAs(004) Bragg backreflection condition for 1 and 1/2 ML InAs samples grown by MBE using the temperature ramp technique. . . 126

7.3 Photon energy dependence of the reflectivity and the In-L FLY near the CaAs(004) Bragg backreflection condition for a series of I ML InAs samples grown by MBE at different growth temperatures. . . . . . . .

7.4 Photon energy dependence of the reflectivity and the In-L FLY near the Ga.As(004) Bragg backreflection condition for 1 and 1/2 .ML In.ts

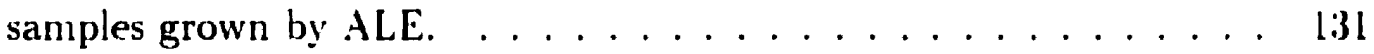

7.5) PL emission from a nominally 1 ML InAs film on $\operatorname{GaAs}(001)$, buried beneath a $2550 \pm 25$ A GaAs cap. . . . . . . . . . . 132

7.6 X-Ray rocking curve data for 1 and 1/2 ML ALE samples obtained near the GaAs(004) symmetric substrate reflection and the results of RADS simulations.

T.i RDS transient signal during three consecutive CiaAs ALE cycles at $400{ }^{\circ} \mathrm{C} \ldots \ldots \ldots \ldots \ldots \ldots \ldots \ldots$

i.8 RDS transient signal during the deposition of 10 GaAs ALE cycles; 1 InAs cycle; and 35 GaAs capping cycles at $460{ }^{\circ} \mathrm{C} . \ldots \ldots \ldots$

i.9 Temperature dependence of the RDS end-of-cycle transients during the deposition of $35 \mathrm{GaAs}$ capping layers by ALE. . . . . . . . . 140

7.10 Temperature dependence of the RDS end-of-cycle fit parameters from the Boltzmann model. . . . . . . . . . . . . . . . . 
7.11 TMGa pulse time dependence of the RDS end-of-cycle transients during the deposition of $35 \mathrm{GaAs}$ capping layers by ALE. . . . . . . . . .

7.12 Photon energy dependence of the reflectivity and the In-L FLY near the GaAs(004) Bragg backreflection condition for a 1 ML InAs ALE sample grown at $400^{\circ} \mathrm{C}$

7.13 AFM topography image for a GaAs reference buffer layer. . . . . . 146

7.14 AFM topography images for SL structures described in the text. . . 148

7.15 Temperature dependence of the RDS signal for GaAs surfaces under TBAs and during GaAs growth. . . . . . . . . . . . .

7.16 $2.6 \mathrm{eV}$ RDS transients during single SL cycles at various growth tem-

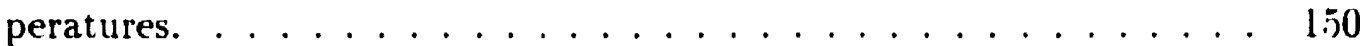

$7.172 .6 \mathrm{eV}$ RDS transients for the first 10 periods of the SL growth at $500^{\circ} \mathrm{C} .151$

7.18 Multitransient energy spectra representing the development of surface anisotropies for the growth of a single $\mathrm{SL}$ period at $4.500^{\circ} \mathrm{C} \ldots \ldots$

7.19 Multitransient energy spectra representing the development of surface anisotropies for the growth of a single SL period at $600^{\circ} \mathrm{C} \ldots \ldots$. . . 153

7.20 XRD results for SL structures near the $\operatorname{GaAs}(004)$ reflection. . . . . . 156

7.21 HRXRD results for $1 \mathrm{ML}$ samples with several growth temperatures, near the GaAs(004) reflection. and the results of dynamical simulations. 1.58

7.22 HRXRD results for 1/2 ML samples with several growth temperatures. near the $\mathrm{GaAs}(004)$ reflection, and the results of dynamical simulations. 159

7.23 Photon energy dependence of the reflectivity and the In-L FLY near the GaAs(004) Bragg backreflection condition for a series of 1 ML InAs MOVPE samples. . . . . . . . . . . . . . . . . . . . . . 161

7.24 Photon energy dependence of the reflectivity and the In-L FLY near the GaAs(004) Bragg backreflection condition for a series of 1/2 ML InAs MOV'PE samples. . . . . . . . . . . . . . . . . . . 162

7.25 The distribution of $\mathrm{In}$ atoms relative to the $\mathrm{GaAs}(004)$ planes according to the two models including segregation effects in the XSW analysis. .

7.26 XSW parameters as a function of segregation coefficient as predicted by model (i) in the text. 
7.27 XSW parameters as a function of segregation coefficient as predicted by model (ii) in the text. . . . . . . . . . . . . 166

7.28 Experimental reflectivity and the In $K_{\alpha}$-fluorescence yield, measured at $28140 \mathrm{eV}$ in the $E_{\|}$-polarization. for the $1 \mathrm{ML}$ InAs SQW grown at $500^{\circ} \mathrm{C} \ldots \ldots$

7.29 In K-edge XAFS from an InAs powder sample and the AUTOBK background.

7.30 Fourier transform magnitude of the $k$-weighted In $k$-edge EXAFS from the InAs powder sample. . . . . . . . . . . . . . . . . . .

T.31 a) Raw EXAFS data from the InAs powder specimen and the FFFS contribution, due to In-As bonds. b) FFFS data and the fit to the data obtained with FEFF predictions.

7.32 In K-edge XANES from the 1 ML InAs films and the InAs reference powder.

7.33 Fourier transform magnitudes for $k$-weighted In $k$-edge EXAFS in $E_{\|}$

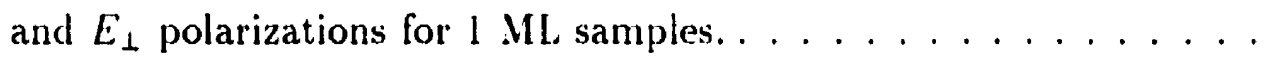

$7.34 k$-weighted In K-edge EXAFS with $E_{\|}$-polarization from 1 ML InAs films at different growth temperatures and from an InAs reference powder in transmission. Superimposed on the data are the fits to the FFFS contributions, corresponding to In-As near neighbour bonds. . . . . .

$7.35 k$-weighted In K-edge EXAFS with $E_{\perp}$-polarization from $1 \mathrm{ML}$ InAs films at different growth temperatures. Superimposed on the data are the fits to the FFFS contributions, corresponding to In-As near neighbour bonds.

7.36 PL spectra for a series of $1 \mathrm{ML}$ InAs samples, with different growth temperatures.

7.37 PL spectra for a series of $1 / 2$ ML InAs samples, with different growth temperatures.

7.38 Summary of the XSW and PL results for the MOVPE samples.

7.39 X-Ray rocking curve data obtained near the $\operatorname{In} P(004)$ symmetric substrate reflection for the strained $\ln _{x} \mathrm{Ga}_{1-x}$ As films. 
7.40 X-Ray rocking curve data obtained near the $\operatorname{In} \mathrm{P}(004)$ reflection at different positions on the $\mathrm{LM} \operatorname{In}_{x} \mathrm{Caa}_{1-x}$ As film. . . . . . . . . . 194

7.41 Indium composition in the input vapour phase compared with the composition determined by XRD and EXAFS measurements. . . . . . 1955

$7.42 k^{2}$-weighted Ga li-edge EXAFS from the GaAs and GaP powders. . 196

$7.43 k^{2}$-weighted As Kiedge EXAFS from the GaAs and InAs powders. . . 197

7.44 The magnitudes of the Fourier transforms of the $k^{2}$-weighted Ga and As K-edge EXAFS from the reference powders. . . . . . . . 198

$7.45 k^{2}$-weighted Ga K-edge EXAFS from pseudomorphic $\operatorname{In}_{r} \mathrm{Ga}_{1-r}$.As alloys

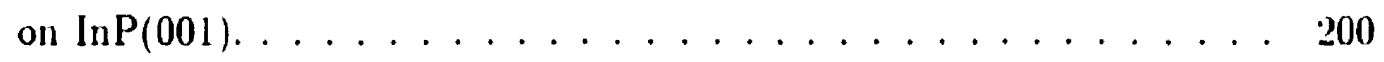

$7.46 k^{2}$-weighted As K-edge EXAFS from pseudomorphic $\operatorname{In}_{x} \mathrm{Ga}_{1-r} . \mathrm{As}$ alloys

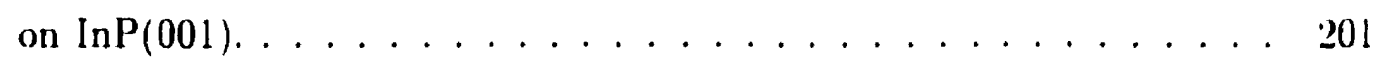

$i .47$ The magnitudes of the Fourier transforms of the $k^{2}$-weighted (ia and As K-edge EXAFS from the ternary alloys. . . . . . . . . . . 203

7.48 Comparison between the experimentally-determined $\mathrm{In}-\mathrm{As}$ and $\mathrm{Ga}-\mathrm{As}$ bond lengths in pseudomorphic $\operatorname{In}_{x} \mathrm{Ga}_{1-x}$ As alloys on $\ln \mathrm{P}(001)$ and the results of the random cluster calculation. . . . . . . . . . . 204

A.1 The crystal structure of zincblende compound semiconductors. . . . 210

A.2 The real and imaginary parts of the Ga. As. and In atomic form factors.212 


\section{List of Abbreviations}

- ADC - Analog-to-digital converter.

- AES - Auger electron spectroscopy.

- AFM - Atomic force microscopy.

- ALE - Atomic layer epitaxy.

- ALMBE - Atomic layer molecular beam epitaxy.

- BC - Boundary condition.

- BNL - Brookhaven National Laboratory.

- CB - Conduction band.

- CBE - Chemical beam epitaxy.

- CTR - Crystal truncation rod.

- $\mathrm{CW}$ - Continuous wave.

- DAFS - Diffraction anomalous fine structure.

- DAP - Donor-acceptor pair.

- DTXRD - Dynamical theory of X-Ray diffraction.

- EELS - Electron energy loss spectroscopy.

- EDR - Enhanced dynamic range.

- EDX - Energy-dispersive X-Ray spectroscopy.

- ETB - Empirical tight-binding.

- EXAFS - Extended X-Ray absorption fine structure.

- FFFS - Fourier-filtered first shell.

- FFT - Fast Fourier transform.

- FLY - Fluorescence yield.

- FME - Flow-rate modulation epitaxy. 
- FT - Fourier transform.

- FWHM - Full-width at half-maximum.

- GID - Grazing incidence diffraction.

- GIXAS - Grazing incidence X-Ray absorption spectroscopy.

- GLXS - Crazing incidence X-Ray scattering.

- HREM - High-resolution electron microscopy.

- HRXRD - High-resolution X-Ray diffraction.

- HVPS - High-voltage power supply.

- LDA - Local density approximation.

- LM - Lattice-matched.

- MC - Monochromator.

- MCA - Multichannel analyzer.

- MEE - Migration-enhanced epitaxy.

- MET - Macroscopic elastic theory.

- MFC - Mass flow controller.

- ML - Monolayer.

- MOCVD - Metalorganic chemical vapour deposition.

- MOVPE - Metalorganic vapour phase epitaxy.

- MQW - Multiple quantum well.

- MS - Multiple scattering.

- NIM - Nuclear instruments module.

- NIST - National Institute of Standards and Technology (United States).

- NSLS - National Synchrotron Light Source.

- NLLSQ - Nonlinear least-squares.

- PL - Photoluminescence.

- PLE - Photoluminescence excitation.

- PSPD - Position-sensitive photodetector.

- QW - Quantum well.

- RADS - Rocking curve analysis by dynamical simulation.

- RBS - Rutherford backscattering.

- RDS - Reflectance difference spectroscopy. 
- RHEED - Reflection high-energy electron diffraction.

- SCA - Single channel analyzer.

- SI - Semi-insulating.

- SIMS - Secondary ion mass spectroscopy.

- SL - Superlattice.

- SS - Single scattering.

- SSRL - Stanford Syuchrotron Radiation Laboratory.

- SQW - Single quantum well.

- TBAs - Tertiarybutylarsine.

- TBP - Tertiarybutylphosphine.

- TEGia - Triethylgallium.

- TEY - Total electron yield.

- TDOA - Thermally detected optical absorption.

- TMCia - Trimethylgallium.

- T.MIn - Trimethylindium.

- UHP - Ultrahigh purity.

- UHV - Uiltrahigh vacuum.

- ITPS - Ultraviolet photoelectron spectroscopy.

- VB - Valence band.

- VCA - Virtual crystal approximation.

- VFF - Valence force field.

- VGF - Vertical gradient freeze.

- XAFS - X-Ray absorption fine structure.

- XANES - X-Ray absorption near edge structure.

- XPS - X-Ray photoelectron spectroscopy.

- XRD - X-Ray diffraction.

- XSW - X-Ray standing wave. 


\section{Chapter 1}

\section{Introduction}

The electronics industry is dominated by products based on elemental semiconductor (silicon) technology. For light-emitting devices. however. compound semiconductors reign. Here bandgap-engineered materials structures of extraordinary complexity are now routinely produced and are gaining share in a market which is set to exceed $\$ 1$ trillion U.S. by the end of the twentieth century. These developments have been achieved through advances in semiconductor growth techniques such as metalorganic vapour phase epitaxy (MOVPE) and molecular beam epitaxy (MBE). For III- $V$ compound semiconductors a myriad of possible structures can be produced by depositing lavers of well-defined thickness and composition upon a substrate crystal which provides a structural "template" for the growth.

A single quantum well ( $\mathrm{SQW}$ ) of InAs in $\mathrm{GaAs}(00 \mathrm{I})$ constitutes one of the most highly-strained III-V heterostructures. This thesis deals with structures produced at the ultimate limit: a single or half monolayer (ML) of In atoms replaces a layer of $\mathrm{Ga}$ atoms in a GaAs crystal (Figure 1.1). The large lattice mismatch ( $7 \%$ ) between the constituent materials makes these systems ideal for the study of fundamental materials issues [1]-[4]. With even fractional ML In depositions, sharp and intense photoluminescence (PL) emission has been observed [5]. Additionally, very recent theoretical [6] and experimental [7] studies have re-emphasized the technological promise of InAs SQWs for highly efficient excitonic lasing.

For these ultrathin InAs layers buried in $\mathrm{GaAs}$ there is considerable interest in 


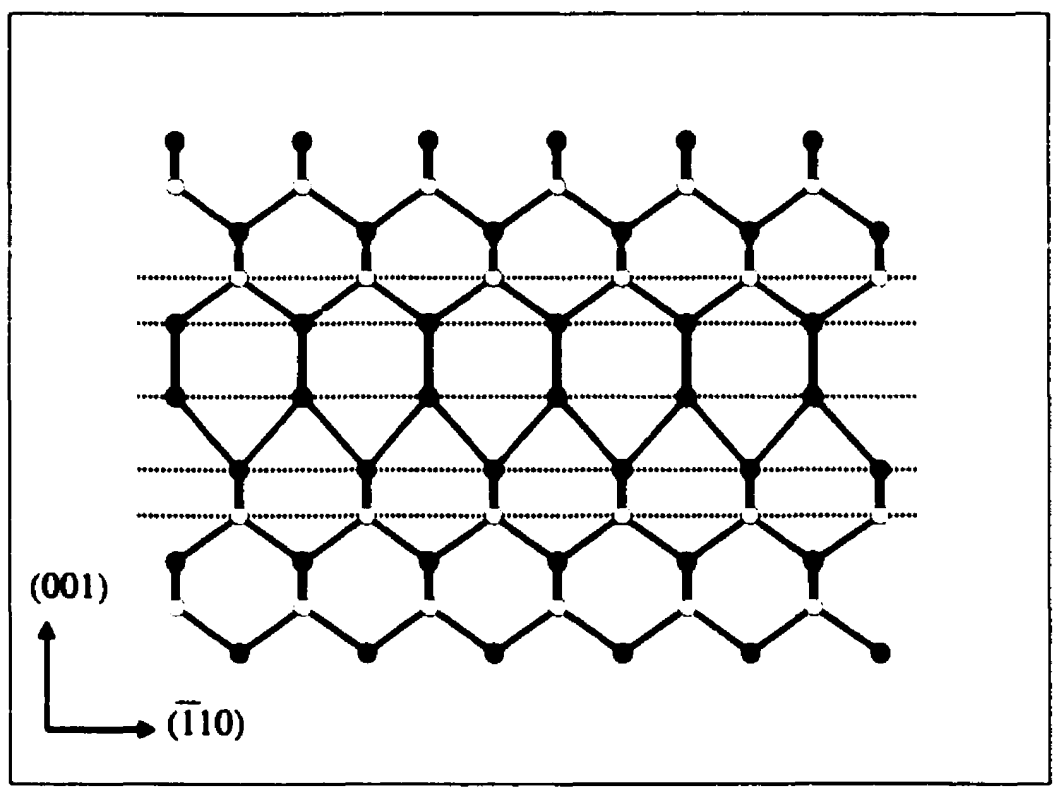

Figure 1.1: A single layer of In atoms embedded in a CaAs(001) crystal. The dotted lines indicate (004)-planes. The In. $\mathrm{Ga}$ and $\mathrm{As}$ atoms are indicated by black, white and shaded circles, respectively.

determining the effect of the In distribution on the optical properties. The challenges involved in this study are manifold. Epitaxy of $\delta$-doping layers of this type requires extremely accurate control of the growth parameters. Researchers have observed In segregation during the topmost GaAs layer deposition using a variety of surface-analytical, optical and structural techniques [8,9]. Suppression or elimination of this $\mathrm{In} / \mathrm{Ga}$ exchange is a principal requirement for the successful fabrication of "perfect" $\delta$-layers. Subsequently, powerful characterization techniques are required to assess the epitaxial structures. Buried interfaces present an experimental challenge since the structural morphology can not be determined using standard surface science techniques.

Recently many reports have examined ultrathin InAs SQWs in GaAs including optical and structural investigations $[5,10]$ and characterization of laser diodes $[\bar{i}, 11$, 
12]. With reduced dimensionality there is a huge increase in the density of states: from delocalized electron properties to increasingly atom-like behaviour. 0-d (quantum dot) confinement is widely believed to be capable of producing large improvements in laser diode performance: the lasing threshold can be greatly reduced by increasing the electron-hole overlap. PL studies have suggested that subML systerns of InAs in GaAs form self-organized 0 -d or 1-d structures during epitaxial growth [13]-[15]. although these optical studies do not provide a detailed description of the In distribution parallel or perpendicular to the growth plane.

In this work InAs SQW structures of 1 and 1/2 ML thickness were produced by MOVPE and atomic layer epitaxy (ALE). In conventional MOVPE, group III and group $\vee$ metalorganic precursors enter the growth chamber simultaneously. Surface reactions at the heated substrate lead to dissociation of the metal atoms from the organic components. resulting in epitaxial growth. ALE is a variation of MOVPE in which the group III and group $V$ precursors enter the chamber separately. with a hydrogen purge between each step. Layer-by-layer growth with ALE was supposed to produce sharp interfaces through self-limiting growth mechanisms [16].

For industrial applications, MOVPE and ALE. typically using pressures of $\sim 50$ Torr. have distinct advantages over $\mathrm{MBE}$ which requires ultrahigh vacuum (LHV). However, MOVPE is less mature than MBE in the in-situ monitoring of surface reactions because electron probe techniques. such as reflection high energy electron diffraction (RHEED), are prohibited at MOVPE pressures. In this work in-situ characterization was achieved using reflectance difference spectroscopy (RDS), which is sensitive to the presence and orientation of atomic surface reconstructions. For both MOVPE and ALE, detailed data describing the surface evolution during SQW growth sequences were obtained. For the MOVPE films, a novel technique exploited the reproducibility of multiple quantum well (MQW) growth cycles to obtain real-time spectroscopic data with extremely high temporal resolution.

High-resolution X-Ray diffraction (HRXRD) allows the determination of film thicknesses using the Pendellösung effect $[10,17]$. This technique accurately determines the total dosage of InAs, but is rather insensitive to details of the In distribution. Highresolution electron microscopy has been used to study the cross-sectional properties 
of MBE structures [18], but cannot resolve interfacial variations at the atomic scale. $\mathrm{X}$-Ray absorption fine structure (XAFS) and X-Ray standing wave (XSW) measurements have been made on InAs MLs grown by MBE and have been successful in determining the perpendicular distance of In atoms relative to the Ga.As (004) planes of the substrate (coherent position). the $\delta$-like nature of the In distribution along the growth direction (coherent fraction), and the In-As bond lengths [2]-[4]. Another study considered the effect of segregation on the measured XSW parameters [1].

In this work. complementary short-range-order (XAFS) and long-range-order techniques (XRD. XSW) including element specificity (XAFS. XSW) were used to provide a detailed structural characterization and to furnish insight into the measured PL properties of these systems. Synchrotron X-Ray measurements were made at the Stanford Synchrotron Radiation Laboratory (SSRL) and the National Synchrotron Light Source (NSLS) at Brookhaven National Laboratory (BNL). The high photon flux provided by synchotron radiation is essential for high-resolution studies of this type. Synchrotron X-rays are also preferred due to their intrinsic collimation, polarization. and their plane-wave-like properties. This work represents the first application of these techniques to MOVPE and ALE samples and the most comprehensive XSW and XAFS study of the effect of growth conditions on the In distribution.

This thesis is divided into eight chapters. Chapter 2 describes general aspects of the MOVPE and ALE techniques. Chapter 3 is devoted to dynamical diffraction in perfect crystals. The chapter begins with the development of the dynamical diffraction theory, and then considers the principles of XSW. Analysis of HRXRD data forms an integral part of the characterization process, and the theoretical background for these simulations is given in the last section of the chapter. Chapter 4 defines the XAFS and gives the mathematical expression used to analyze the experimental data. The electric field amplitudes of the waves travelling through layered structures during glancing-incidence reflection are considered using a recursive method. It is shown that this geometry also leads to an XSW field, which in this case extends far above the sample surface.

Chapter 5 summarizes the literature to provide a firm foundation in the theoretical and experimental precedents for this work. This includes discussions of strain, 
structure and segregation in InAs SQWs in GaAs. RDS literature and optical studies of InAs SQWs are also briefly reviewed. In Chapter 6 a detailed description is given of the experimental apparatus used in the different components of this study, at SFL. SSRL and the NSLS. The experimental data are presented in Chapter $i$ which is divided into sections devoted to analysis and interpretation of studies on several groups of samples. Specifics of the sample preparation appear in each section. A more general study of strained bond lengths in $\ln _{r} \mathrm{Ga}_{1-r}$. As films grown on $\operatorname{In} \mathrm{P}(001)$ is also reported. Finally, important conclusions drawn from this work are summarized in Chapter 8. together with suggestions for future directions to be taken in this area of research. 


\section{Chapter 2}

\section{Metalorganic Vapour Phase Epitaxy}

For epitaxial growth of thin-film semiconductors two dominant techniques have emerged. MOVPE and MBE. The former was originally called metalorganic chemical vapour deposition (MOCVD) by Manasevit who pioneered the technique in the late sixties [19]. International conferences have adopted the MOVPE acronym. which emphasizes the epitaxial nature of the process.

As the name suggests, MOVPE involves the transport of metalorganic precursor molecules to a heated single crystal substrate. At the vapour/solid interface complex chemical reactions occur with two principal results: the decomposition of the precursor molecules and the incorporation of metal atoms into the crystal. The latter process usually involves the arrangement of the metal atoms in registry with the single crystal matrix. For zincblende compound semiconductors, which are exclusively studied in this work, the group III and group $V$ atoms provided by the precursors reside in their appropriate positions in the epitaxial film and continue the lattice structure of the substrate.

The MBE process is similar, but involves the use of elemental sources, with the process occurring in an ultrahigh vacuum ambient. In this respect, MBE is more challenging than MOVPE, which can produce very good results over a wide range of pressures from 1 atmosphere downwards. 
For heteroepitaxy, the process is more complicated. The deposited thin film does not necessarily contain the same atomic species as the substrate, and may thus have a different native lattice constant from that of the substrate. Epitaxial films with larger lattice dimensions than the substrate would then experience a compressive biaxial strain in order to maintain registry with the substrate. Likewise. films with dimensions smaller than the substrate undergo tensile strain. This aspect of the heteroepitaxy must be carefully considered in the selection of appropriate materials for given device applications. Strain in MOVPE-grown films will form a major component of this thesis and will be discussed in some detail in Section 5.1.

In this chapter the MOVPE process will be described. Beginning with a summary of precursor containment and delivery to the reactor. details of the molecular decomposition at the crystal surface will be described. We can then discuss the incorporation of metal atoms into the crystal.

\subsection{Source Precursors}

Metalorganic source chemicals used in this work are contained in stainless steel "bubblers". shown schematically in Figure 2.1. The carrier gas, in this case palladiumpurified hydrogen. flows into the inlet of the bubbler, which is maintained at a const ant pressure. The source chemicals are usually in liquid form although the indium source employed in this work, trimethylindium (TMIn), $\left(\mathrm{CH}_{3}\right)_{3} \mathrm{In}$. is solid at room temperature. In either case the $\mathrm{H}_{2}$ gas delivers precursor molecules from the vapour phase towards the reactor. where the growth ultimately proceeds. In the SFU facility, both group III and group V elements are supplied by metalorganic sources of this type. Traditionally, the group $V$ elements were supplied by hydride sources such as arsine $\left(\mathrm{AsH}_{3}\right)$. Among the advantages of the metalorganic group $V$ sources (e.g. tertiarybutylarsine (TBAs), $\left(\mathrm{C}_{4} \mathrm{H}_{9}\right) \mathrm{AsH}_{2}$; tertiarybutylphosphine (TBP), $\left.\left(\mathrm{C}_{4} \mathrm{H}_{9}\right) \mathrm{PH}_{2}\right)$ are reduced toxicity, improved safety and lower decomposition temperatures. Two gallium sources were used in this work, triethylgallium (TEGa), $\left(\mathrm{C}_{2} \mathrm{H}_{5}\right)_{3} \mathrm{Ga}$ and trimethylgallium (TMGa), $\left(\mathrm{CH}_{3}\right)_{3} \mathrm{Ga}$.

The partial pressure of the precursor vapour in the bubbler depends on the bubbler 


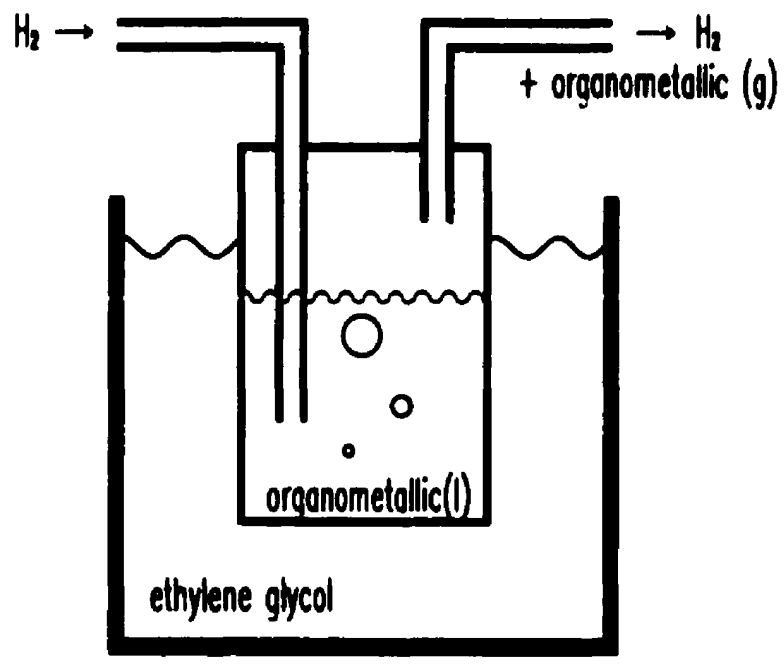

Figure 2.1: Prepurified hydrogen gas flowing through a stainless steel bubbler containing liquid source metalorganic molecules. The saturated vapour is transported from the bubbler outlet downstream towards the reactor vessel. (From Arès [16]).

\begin{tabular}{|c|c|c|c|c|c|c|}
\hline \hline Precursor & $a$ & $b(K)$ & $T_{b}(\mathrm{~K})$ & $P_{b}$ (Torr) & $P_{\text {( Torr })}$ & $f_{P}^{\prime}(\mu \mathrm{mol} / \mathrm{min} / \mathrm{sccm})$ \\
\hline TMGa & 8.501 & 1824 & 263 & 1200 & 37.1 & 1.42 \\
\hline TEGa & 9.172 & 2532 & 291 & 780 & 2.99 & 0.172 \\
\hline TMIn & 10.52 & 3014 & 291 & 800 & 1.47 & 0.08223 \\
\hline TBAs & 7.243 & 1509 & 280 & 800 & 71.9 & 4.41 \\
\hline TBP & 7.586 & 1539 & 280 & 800 & 12.4 & 8.17 \\
\hline \hline
\end{tabular}

Table 2.1: Material parameters for precursors used in this work.

temperature according to

$$
P_{l}(T o r r)=10^{\left[a-b / T_{b}\right]},
$$

where the bubbler temperature, $T_{b}$, is measured in degrees lielvin. Bubbler temperatures are maintained using temperature baths as shown in Figure 2.1. The constants $a$ and $b$ may be found from manufacturers' specifications for a given source. ${ }^{\prime}$ Relevant data for sources used in this work appear in Table 2.1.

The flow rate of hydrogen into the bubbler is adjusted by mass flow controllers (MFCs) which allow a stable quantity of gas to flow per unit time. The molar flow

\footnotetext{
'e.g. Epichem, Inc. product literature
} 
rate, $f_{P}$, is then found from the relation

$$
\int_{P}=\frac{F}{C^{\prime}}\left(\frac{P_{l}}{P_{b}-P_{l}}\right) \text {. }
$$

Here $P_{b}$ is the bubbler pressure and $C=22407.47$ is the volume in cc of 1 mole of an ideal gas at STP. F represents the volume flow rate in standard cubic centimetres per minute $(\mathrm{sccm})$. Defining the quantity $f_{P}^{\prime} \equiv f_{P} / f^{\prime}$. we find calculated values as shown in Table 2.1. Figure 2.2 illustrates the structure of two of the precursor molecules used in this work.

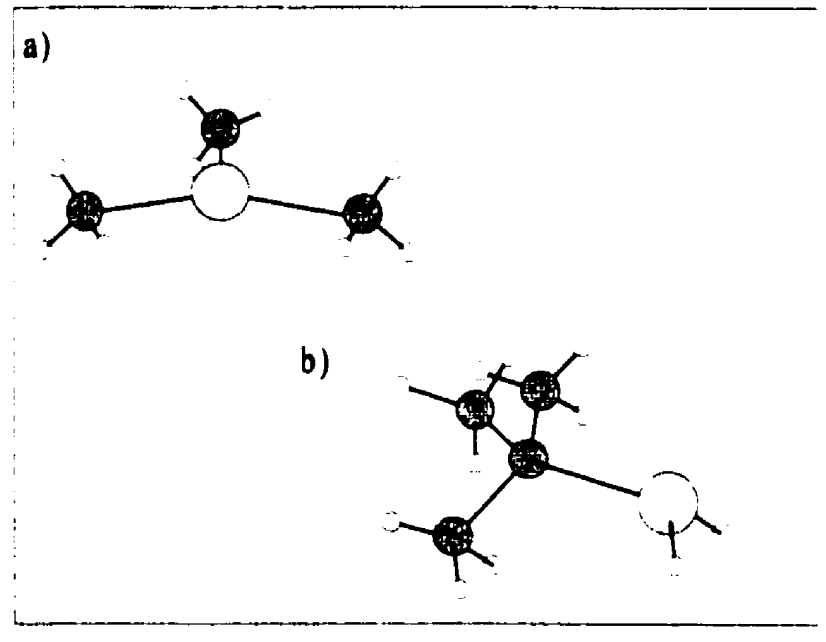

Figure 2.2: Precursor molecules: a) trimethylgallium b) tertiarybutylarsine. The large grey circles, smaller grey circles and empty circles represent metal atoms. $\mathrm{C}$ atoms and $\mathrm{H}$ atoms, respectively.

\subsection{Reactor Flows}

The saturated hydrogen gas transports the precursor molecules to the reactor through stainless steel tubing at ambient temperature. To prevent condensation of the precursor molecules inside these tubes, source chemicals must meet the requirement of a relatively high vapour pressure at temperatures at or below ambient. Moreover, reactions between group III and group V molecules prior to their arrival at the substrate 
are to be avoided. To this end. the Thomas Swan MOVPE system employed in this work features separate lines for group III and group V gas transport to the reactor.

For stable molecular flows required to achieve reproducible results a number of factors must be considered. The bubbler temperatures and pressures must be accurately maintained, according to the discussion of the previous section. As well. the MFCs must supply a well-defined quantity of gas through the bubblers. This is achieved in part by operating the MFC's in a range from about $5 \%$ to $100 \%$ of their full scale supplies. This avoids the lower range where the flow might be most susceptible to small fluctuations. Additionally, the MFC flows are allowed to stabilize over a period of 5 to 20 minutes before initiating the precursor flow to the reactor. The Thomas Swan system employs a run-to-vent switching scheme which diverts the flow of a given species from the reactor, while allowing the flow to stabilize. The flow may then be rapidly directed towards the reactor at the appropriate time in the growth cycle. Details of the system are described in Section 6.1 .

A very short distance from the reactor chamber. the metalorganic vapours are mixed together in a nozzle to promote uniformity. The mixture then travels towards the substrate. Within the reactor, complex hydrodynamic processes occur. Ideally. one wants a steady, laminar gas flow with few eddy currents or recirculating flows. In practice. this is difficult to achieve. Computer simulations have been developed to address this issue [20]. Alternatively, several guidelines exist for successful reactor design [21]. These involve avoiding the presence of sudden changes in reactor diameter. the presence of obstacles and recirculating convection currents due to the heating of the gases at the substrate. These factors can all limit the ability of reactors to produce uniform growth rates and abrupt changes in solid composition.

For the growth of InAs films in GaAs in this work, a vertical reactor geometry was employed. Although these reactors can produce outstanding results. it has been noted [21] that recirculating regions may be produced. These can enhance premature reactions and make rapid change of gas-phase composition impossible. A solution which is now commonly employed (but was not adopted in this work) involves spinning the susceptor rapidly to sweep the gas across the substrate. In this work a low pressure of 50 Torr was used to minimize the eddy currents in the cell. A schematic of the 
vertical reactor cell appears in Figure 2.3.

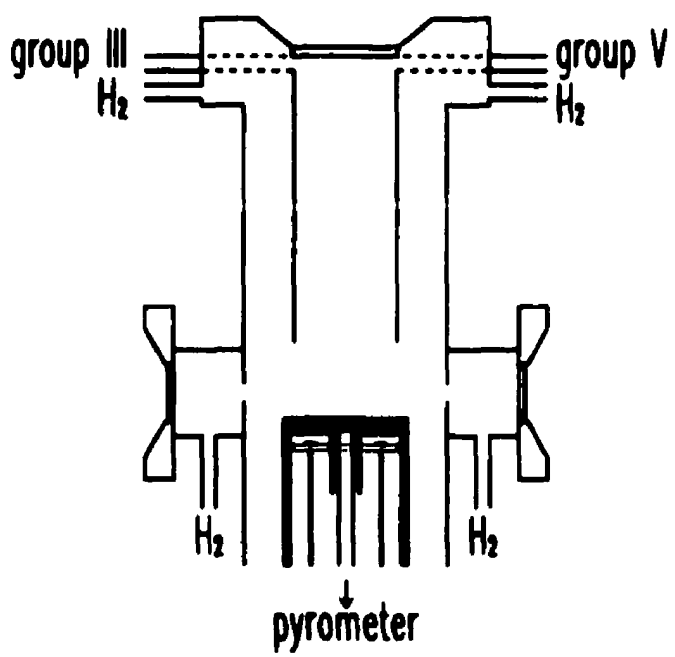

Figure 2.3: The vertical reactor cell employed in this work. Ciases travel downwards from the mixing nozzle towards the substrate which sits upon the heated susceptor. The susceptor temperature is measured by an infrared optical pyrometer which views the bottom of the susceptor. This temperature is regularly calibrated using a thermocouple placed directly on the top susceptor surface.(From Arés [16]).

Directly above the substrate a region exists where the gas flow is directed parallel to the sample surface. The transport of precursor molecules to the growing surface is achieved exclusively by the diffusion of the molecules through this "boundary layer" region. In the boundary layer there is no significant movement of the carrier gas perpendicular to the sample surface. This aspect of MOVPE is remarkably different from the MBE process which involves simple, ballistic transport of source molecules towards the substrate.

\subsection{Precursor Decomposition}

Within the boundary layer, complex chemical reactions result in the dissociation of the source molecules into new species, and provide the necessary metal atoms to the growing surface. The reactions may be classified as homogeneous or heterogeneous. 
depending on whether molecules of different types interact separately, or with one another in the decomposition.

Pyrolysis of different precursor molecules is discussed at length in Reference [21]. Conflicting mechanisms are sometimes deduced and point to the difficulty in uncovering the details of processes occurring in the "messy" MOVPE environment. We consider the decomposition of the source compounds used in this work.

TMGa (see Figure 2.2) and TMIn decompose by a simple homolytic fission reaction producing methyl radicals. The reaction is thought to proceed in three steps:

$$
\begin{aligned}
\left(\mathrm{CH}_{3}\right)_{3} \mathrm{Ga} & \rightarrow \mathrm{CH}_{3}+\mathrm{Caa}\left(\mathrm{CH}_{3}\right)_{2} \\
& \rightarrow 2 \mathrm{CH}_{3}+\mathrm{Ga}\left(\mathrm{CH}_{3}\right) \\
& \rightarrow 3 \mathrm{CH}_{3}+\mathrm{Ga}
\end{aligned}
$$

Methyl radicals produced in these reactions are removed from the surface by $\mathrm{H}$ atoms. which result from the decomposition of the group $V$ precursors. as will be discussed. For TWIn. the pyrolysis occurs at fairly low temperatures, with $100 \%$ decomposition achieved as low as $\sim 350^{\circ} \mathrm{C}$.

TEGa pyrolyzes homogeneously to produce diethylgallane and ethene molecules through the following so-called 3 -elimination reaction:

$$
\left(\mathrm{C}_{2} \mathrm{H}_{5}\right)_{3} \mathrm{Ga} \rightarrow \mathrm{C}_{2} \mathrm{H}_{4}+\left(\mathrm{C}_{2} \mathrm{H}_{5}\right)_{2} \mathrm{GaH}
$$

Complete decomposition by this mechanism occurs by about $350{ }^{\circ} \mathrm{C}$.

The decomposition mechanisms for the tertiarybutyl compounds are slightly more complex. An advantage of these sources is that decomposition occurs at lower temperatures than for the hydrides, allowing greater flexibility in determining suitable temperatures for a given process. Several possible reaction mechanisms exist:

$$
\begin{array}{lll}
\left(\mathrm{C}_{4} \mathrm{H}_{9}\right) \mathrm{AsH}_{2} & \rightarrow \mathrm{C}_{4} \mathrm{H}_{9}+\mathrm{AsH}_{2} & \text { (i) } \\
\text { or } & \rightarrow \mathrm{C}_{4} \mathrm{H}_{10}+\mathrm{AsH} & \text { (ii) } \\
\text { or } & \rightarrow \mathrm{C}_{4} \mathrm{H}_{8}+\mathrm{AsH}_{3}
\end{array}
$$

There is compelling evidence [21] that tertiarybutylphosphine (TBP) decomposes according to the following mechanisms which seem to occur with no dependence on the group III source. 


\begin{tabular}{|c|c|c|c|c|}
\hline \hline \% Decomposition & $\mathrm{AsH}_{3}$ & $\mathrm{TBAs}$ & $\mathrm{PH}_{3}$ & $\mathrm{TBP}$ \\
\hline 25 & 650 & 280 & 600 & 420 \\
\hline 50 & 680 & 300 & 700 & 450 \\
\hline 75 & 700 & 350 & 800 & 475 \\
\hline 100 & 760 & 475 & 850 & 550 \\
\hline \hline
\end{tabular}

Table 2.2: Decomposition profiles of hydride and tertiarybutyl group $V$ sources. Tell1peratures are given in ${ }^{\circ} \mathrm{C}$. From Air Products and Chemicals. Inc. product literature.

$$
\begin{array}{ll}
\left(\mathrm{C}_{4} \mathrm{H}_{9}\right) \mathrm{PH}_{2} & \rightarrow \mathrm{C}_{4} \mathrm{H}_{9}+\mathrm{PH}_{2} \\
\mathrm{C}_{4} \mathrm{H}_{9}+\left(\mathrm{C}_{4} \mathrm{H}_{9}\right) \mathrm{PH}_{2} & \rightarrow \mathrm{C}_{4} \mathrm{H}_{10}+\mathrm{C}_{4} \mathrm{H}_{9} \mathrm{PH} \\
\mathrm{C}_{4} \mathrm{H}_{9} \mathrm{PH} & \rightarrow \mathrm{C}_{4} \mathrm{H}_{9}+\mathrm{PH} \\
\mathrm{C}_{4} \mathrm{H}_{9} & \rightarrow \mathrm{C}_{4} \mathrm{H}_{8}+\mathrm{H}
\end{array}
$$

The tertiarybutyl sources have temperature decomposition profiles as indicated in Table 2.2. From the table it is clear that the organic sources have advantageous properties when compared with the hydrides.

\subsection{Incorporation into the Solid}

From the boundary layer. the metal atoms must be adsorbed to the solid surface for epitaxy to proceed. There are in fact several phenomena occuring simultaneously: adsorption. desorption, surface diffusion. surface reactions, and attachment at kinks on step edges [21].

The epitaxy itself is driven by thermodynamics at the solid-vapour interface. Briefly, the supersaturation of the vapour drives the system from equilibrium between the solid and vapour phases. The system strives to reduce the difference in chemical potentials of the two phases.

$$
\Delta \mu=\mu_{v}-\mu_{s} .
$$

where $\mu_{v}$ and $\mu_{s}$ are the chemical potentials of the vapour and solid phases, respectively. The premise is elucidated succinctly by Stringfellow: 
"A nonequilibrium situation is intentionally created that drives the system to produce the solid desired." ${ }^{2}$

Several other factors are involved besides thermodynamics. Kinetics define the rates at which processes occur. while hydrodynamics and mass transport control the rate of material transport to the growing solicl-vapour interface. It is often possible in deternine the rate-limiting step in a given growth process by examining the temperature dependence of the growth rate. $R_{y}$. defined as the thickness of material deposited per unit time. An illustration of the various regimes is given in Figure 2.4.

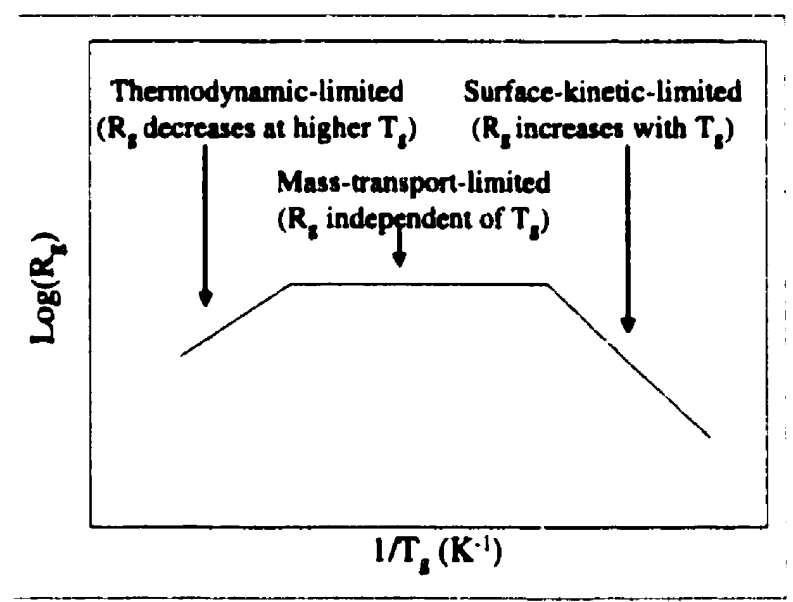

Figure 2.4: Different regimes of epitaxy according to the growth temperature. $T_{y}$.

In conventional MOVPE. the growth is usually carried out in the mass-transportlimited region. Here the growth rate is independent of temperature. but depends linearly on the total flow. In ALE. the growth is necessarily surface-kinetic-limited. This technique will be discussed in more detail in Section 2.5.

In the mass-transport-limited regime, for many materials the growth rate is independent of the group $V$ flow and depends only on the group III flow. In this situation essentially all of the group III atoms arriving at the growing surface are incorporated into the crystal from an excess group $V$ vapour environment. This is not the case for

\footnotetext{
${ }^{2}$ Reference [21], p. 106
} 
some alloy materials (e.g. GaAsSb) which require V/III ratios of very close to unity for successful growth [21].

In practice it is not possible to obtain perfectly flat, single-crystal substrates. Even the best available GaAs crystals contain a residual miscut. This means that the crystal surface is misoriented by some small angle with respect to the crystal planes. In this work (001)-oriented substrates were used exclusively. with typical miscut tolerances of $\pm 0.1^{\circ}$ from the exact orientations. With such small misorientations. the sample surface is usually characterized by large. uniform terraces of several hundred or thousand angstroms in width. For example. terraces of 1 ML height on a GaAs(001) surface with $0.1^{\circ}$ misorientation would have widths of $\sim 1621 \mathrm{~A}$. A fundamental mechanism of crystal growth thus involves the adsorption of metal atoms at the edges of atomic steps in the crystal. The step edges have a large number of available bonds and thus provicle favourable sites for chemisorption. "Step flow" conditions, often encountered in MOVPE. refer to the process in which incoming species migrate along the crystal surface towards step edges where adsorption occurs. This results in the continuous growth of the atomic steps, and the propagation of the steps until either coalescence with another step, or an interruption at the edges of a sample. An illustration of the step flow growth mode appears in Figure 2.5.

The structure of the growing surface is actually rather complicated and can involve so-called reconstructions. It is energetically favourable for dangling bonds at the surface to rearrange themselves in their bonding configurations with their neighbours. often atoms of the same species. For instance, As atoms in a GaAs crystal always have four bonds to neighbouring $\mathrm{Ga}$ atoms within the bulk. ${ }^{3}$ On the surface. however. it is common for the As atoms to bond to one another. In the MOVPE GaAs growth sequences employed in this work, the group $V$ molar flow rate is usually many times greater than the group III rate; the V/III ratio is usually quite high. Under these conditions it is common to find As-rich surfaces during growth, where the crystal surface may contain two or more layers of As atoms in some reconstruction. A more complete discussion of surface reconstructions during growth will appear in Section 5.3.

\footnotetext{
${ }^{3}$ An exception is the occupation of a Ga site by an As atom which creates an antisite defect, known as EL2, in the crystal [21].
} 


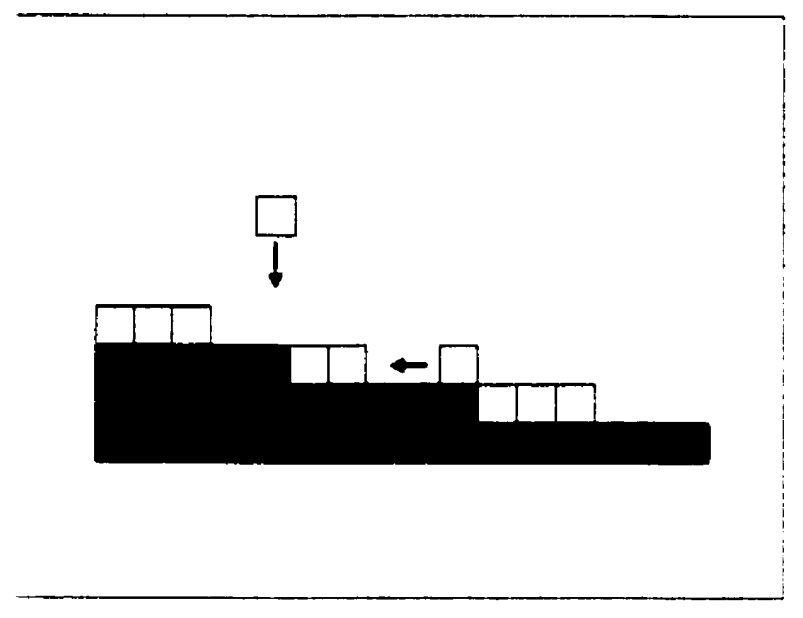

Figure 2.5: A schematic illustration of step flow growth. The deposited adatoms incorporate only at steps.

\subsection{Atomic Layer Epitaxy}

A thorough discussion of ALE growth mechanisms has been given by Ares [16]. In this section a brief discussion of the technique will be presented in connection with the InAs SQW growth sequences described in Sections $7.3-7.5$.

As mentioned, ALE is a variation of the MOVPE technique in which the cation and anion species flow to the crystal surface at separate times. A typical GaAs growth sequence involves a TMGa pulse of a few seconds duration, a $\mathrm{H}_{2}$ purge. a TB.As pulse of a few seconds duration. and a second $\mathrm{H}_{2}$ purge. The goal of this technique is to produce atomically flat interfaces as a result of the high cation mobilities in the absence of an accompanying anion Hux. ALE growth is usually carried out in a "self-limiting" regime. Here the cation species are chemisorbed onto the crystal until the incorporation reaches a level of a single monolayer. No additional material is subsequently adsorbed.

For homoepitaxy, ALE has indeed been able to produce material of exceptional surface quality [22]. There are two main drawbacks to the technique. First, it seems that the chemical mechanisms which permit the self-limiting behaviour rely upon 
methyl radicals bonded to the group III atoms in the top ML of the crystal [16]. While these radicals prevent the adsorption of more than $1 \mathrm{ML}$ of material, they result in the incorporation of excess $\mathrm{C}$ atoms in the crystal. As a result, ALE-grown GaAs using TMGa is usually found to be p-type with carrier concentrations of approximately $10^{18} \mathrm{~cm}^{-3}$. Conventional MOVPE growth of GaAs using TEGa usually results in n-type material, with carrier concentrations of $10^{15} \mathrm{~cm}^{-3}$ or less.

Secondly, the high cation mobilities can contribute to the segregation of one atomic species in heteroepitaxy of quantum wells. for instance. In the absence of a group $V$ flux. the surface is more susceptible to group III atomic rearrangements near the surface. Although one can produce highly uniform and smooth quantum well layers. it is difficult to bury the layers at their nominal positions for use in quantum-well devices. In this work. In atoms deposited on CiaAs surfaces are prone to exchanging positions with incoming $\mathrm{Ga}$ atoms during the GaAs cap layer growth. resulting in In atoms floating atop the growing GaAs laver. This segregation phenomenon presents a challenge for the production of $\delta$-like single ML films of InAs in Cia.ts and will be discussed in detail in Section 5.2.

Having now described the underlying principles of the epitaxial growth methods used in this work, we are in a position to consider the analytical techniques which have been used to characterize the films. For layered structures, a standard laboratory technique is HRXRD which will be described in the next chapter. In this thesis different aspects of the dynamical diffraction are exploited. including the presence of a standing wavefield in perfect crystals during Bragg diffraction. The basis for these techniques will now be presented, followed by a discussion of glancing incidence XAFS. Finally, other in-situ and $e x$-situ techniques will be discussed. 


\section{Chapter 3}

\section{Dynamical X-Ray Diffraction}

For diffraction of X-rays by perfect crystals, such as the epitaxial layers studied in this work, the underlying principles are described by the dynamical theory of $X$-ray diffraction (DTXRD) [23, 24]. In this chapter the development of the theory will be described in a manner which permits the application in the XSW technique, as well as in the HRXRD method. In the latter, a method of solution will be outlined which is attributed to Takagi [25] and Taupin [26] and is the basis for studies of layered structures.

\subsection{Wavefields in Crystalline Media}

For large perfect crystals the diffraction process must be described by considering the electromagnetic wavefield in the crystal as a single entity [2:3]. This distinguishes the dynamical approach from the simpler kinematical description which may be used for powder samples. In the diffraction process the incident and diffracted beams are coherently coupled, allowing energy to be exhanged between them. In the usual picture, diffraction occurs if the Ewald sphere of radius $1 / \lambda$ passes through the origin of the reciprocal lattice and has its centre along the incident beam direction. while passing through a given reciprocal lattice point (Figure 3.1). Here $\lambda$ is the $X$-ray wavelength. This geometry satisfies Bragg's law relating the diffraction angle, $\theta_{B}$. 
and the spacing of the crystal diffraction planes, $d_{\vec{A}}$.

$$
\lambda=2 d_{A} \sin \theta_{B} .
$$

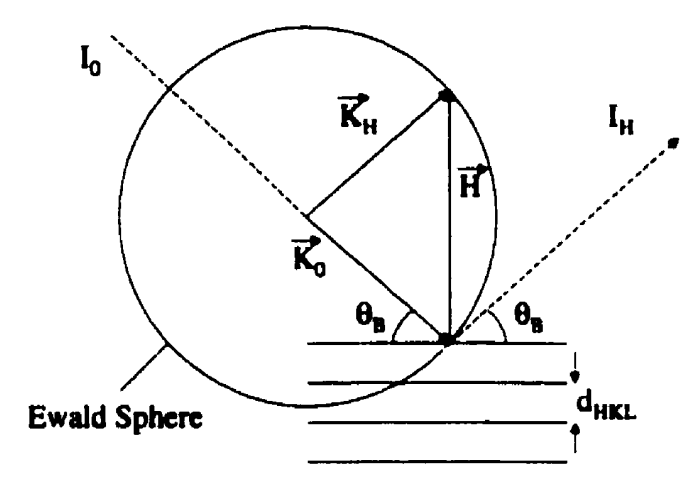

Figure 3.1: The Ewald sphere and Bragg 's law. The incident wave $\left(I_{0}\right)$ with wavevector $\vec{K}_{0}$ is diffracted by the lattice planes with spacing $d_{A}$ and reciprocal lattice vector $\vec{H}$ into the diffracted wave $\left(I_{H}\right)$ with wavevector $\vec{K}_{H}$. The X-Ray wavelength, $\lambda$, is related to the wavevector as $\left\|\vec{K}_{0}\right\|=1 / \lambda$. The figure illustrates the situation in real and reciprocal space.

The basis for the dynamical theory, pointed out by von Laue [2 7 ], is the solution of Maxwell's equations in a medium with a periodic. anisotropic. complex dielectric constant. The electron density at a position $\vec{r}$ in the crystal may be expressed [2:3] as a Fourier sum over the reciprocal lattice as

$$
\rho(\vec{r})=\frac{1}{V} \sum_{H} F_{H} \exp (-2 \pi i \vec{H} \cdot \vec{r})
$$

Here $\mathrm{V}$ denotes the volume of the unit cell and $\vec{H}$ is a reciprocal lattice vector with 
Miller indices $(H K L)$. The structure factor is then given by

$$
F_{H}=\int_{\vec{V}} \rho(\vec{r}) \exp (2 \pi i \vec{H} \cdot \vec{r}) d v=\sum_{n} f_{n} \exp \left(2 \pi i \vec{H} \cdot \vec{r}_{n}\right)
$$

where the sum is over all atoms in the unit cell, and $f_{n}$ is the atomic scattering factor of atom $n$. Details of the calculation of $F_{H}$ for a zincblende semiconductur are given in Appendix $A$. The electric displacement, $\vec{D}$, is defined as

$$
\vec{D}=\Xi \vec{E}=\kappa \Sigma_{0} \vec{E}=\varepsilon_{0} \vec{E}+\vec{P} \text {. }
$$

where $\Xi$ is the permittivity of the material, $\vec{P}$ is the polarization, $\vec{E}$ is the electric field amplitude. and $\Sigma_{0}$ is the permittivity of free space. The dielectric constant of the material is given by

$$
\kappa=\varepsilon / \varepsilon_{0}=1+1 .
$$

where $\downarrow$ is the electric susceptibility. In terms of the electron density. the dielectric constant may be written [2:3] as a function of position

$$
r(\vec{r})=1-r_{e} \frac{\lambda^{2}}{\pi} \rho(\vec{r}) .
$$

where $r_{e}=\epsilon^{2} / 4 \pi \varepsilon_{u} m c^{2}$ is the classical electron radius $\left(2.82 \times 10^{-5} \mathrm{~A}\right)$. It is convenient to define $\Gamma \equiv r_{\mathrm{e}} \lambda^{2} / \pi V$. from which it follows that

$$
\kappa(\vec{r})=1-\Gamma \sum_{H} F_{H} \exp (-2 \pi i \vec{H} \cdot r)
$$

The atomic scattering factor is modified by the anomalous dispersion corrections which include effects of scattering and absorption, i.e. $f_{n}=f_{n}^{0}+f_{n}^{\prime}+i f_{n}^{\prime \prime}$. Hence, $F_{H}=F_{H}^{\prime}+i F_{H}^{\prime}$.

Neglecting the electric displacement current and assuming that the medium has a magnetic permeability equal to that of free space, $\mu=\mu_{0}$, Ampere's law becomes

$$
\nabla \times \overrightarrow{\mathcal{H}}=\frac{\partial \vec{D}}{\partial t}=\varepsilon_{0} \frac{\partial \kappa \vec{E}}{\partial t} \approx \varepsilon_{0} \kappa \frac{\partial \vec{E}}{\partial t},
$$

while Faraday's law is

$$
\nabla \times \vec{E}=-\frac{\partial \vec{B}}{\partial t}=-\mu_{0} \frac{\partial \overrightarrow{\mathcal{H}}}{\partial t}
$$


The solutions for the field vectors, $\vec{E} \cdot \vec{D}$, and the magnetic vector, $\overrightarrow{\mathcal{H}}$, are assumed to have the form of plane waves, i.e.

$$
\vec{E}(\vec{r})=\left[\sum_{H} \vec{E}_{H} \exp (-2 \pi i \vec{H} \cdot \vec{r})\right] \exp \left(-2 \pi i \vec{\kappa}_{0} \cdot \vec{r}\right) \exp (2 \pi i \nu t) .
$$

and sinilarly for $\vec{D}$ and $\overrightarrow{\mathcal{H}}$. where $\nu$ is the frequency of the wave [2:3]. These solutions have the form of Bloch waves in the periodic material. and conservation of momentum has been included through Bragg's law, relating the incident and diffracted wavevectors through the relation

$$
\vec{K}_{H}=\vec{K}_{v}+\vec{H}
$$

Substituting Equation 3.10 into Maxwell's equations, it is found that

$$
\vec{K}_{H} \times \overrightarrow{\mathcal{H}}_{H}=-\nu \vec{D}_{H} .
$$

and

$$
\vec{K}_{H} \times \vec{E}_{H}=\nu \mu_{0} \overrightarrow{\mathcal{H}}_{H} .
$$

These two equations show that $\vec{K}_{H} . \overrightarrow{\mathcal{H}}_{H}$ and $\vec{D}_{H}$ form a mutually orthogonal set.

From Equations 3.4 and 3.7. Equation 3.10 for the displacement vector may be written in terms of $\vec{E}$ as

$$
\sum_{H} \vec{D}_{H} \exp \left(-2 \pi i \vec{K}_{H} \cdot \vec{r}\right)=\Sigma_{0}\left[1-\Gamma \sum_{H^{\prime}} F_{H^{\prime}} \operatorname{\epsilon xp}\left(-2 \pi i \vec{H}^{\prime} \cdot \vec{r}\right)\right] \sum_{H} \vec{E}_{H} \exp \left(-2 \pi i \vec{K}_{H} \cdot \vec{r}\right)
$$

where the index $H^{\prime}$ is used to distinguish it from $H$. Since

$$
\vec{K}_{H^{\prime}}+\vec{H}=\vec{K}_{H^{\prime}+H} \text {. }
$$

Equation 3.14 can be written

$\sum_{H} \vec{D}_{H} \exp \left(-2 \pi i \vec{K}_{H} \cdot \vec{r}\right)=\varepsilon_{0} \sum_{H} \vec{E}_{H} \exp \left(-2 \pi i \vec{K}_{H} \cdot \vec{r}\right)-\varepsilon_{0} \Gamma \sum_{H}\left(\sum_{P} F_{H-P} \vec{E}_{P}\right) \exp \left(-2 \pi i \vec{K}_{H} \cdot \vec{r}\right)$.

after changing the indices of summation [23]. This equation is valid for all positions, $\vec{r}$, and for all Fourier components, so

$$
\vec{D}_{H}=\varepsilon_{0} \vec{E}_{H}-\varepsilon_{0} \Gamma \sum_{P} F_{H-P} \vec{E}_{P}=\varepsilon_{0}\left(1-\Gamma F_{0}\right) \vec{E}_{H}-\varepsilon_{0} \Gamma \sum_{P \neq H} F_{H-P} \vec{E}_{P}
$$


The displacement field is thus dominated by $\varepsilon_{0} \kappa_{0} \vec{E}_{H}$, where $\kappa_{0} \equiv 1-\Gamma F_{0}$, but contains small contributions from other Fourier components of the electric field.

Taking the cross product of $\vec{K}_{H}$ with each side of Equation 3.9 and substituting in Equation 3.8.

$$
\vec{K}_{H} \times\left(\vec{K}_{H} \times \vec{F}_{H}\right)=1 \mu_{0}\left(\vec{K}_{H} \times \overrightarrow{\mathcal{H}}_{H}\right)=-!^{2} \mu_{0} \vec{D}_{H} .
$$

Using the form of the displacement in Equation 3.17, this becomes

$$
\vec{K}_{H} \times\left(\vec{K}_{H} \times \vec{E}_{H}\right)=-\nu^{2} \mu_{0} \tilde{z}_{0}\left(\vec{E}_{H}-\Gamma \sum_{P} F_{H-P} \vec{E}_{P}\right) .
$$

The vacuum value of the wavevector is related to the frequency by $k=\nu / c$, and since $\mu_{0} \varepsilon_{0}=1 / c^{2}$.

$$
\vec{K}_{H} \times\left(\vec{K}_{H} \times \vec{E}_{H}\right)+k^{2} \vec{E}_{H}-k^{2} \Gamma \sum_{P} F_{H-P} \vec{E}_{P}=0
$$

I'sing the vector identity for the triple cross product. this becomes

$$
\left[k^{2}\left(1-\Gamma F_{0}\right)-\left(\vec{K}_{H} \cdot \vec{K}_{H}\right)\right] \vec{E}_{H}-k^{2} \Gamma \sum_{P \neq H} F_{H-P} \vec{E}_{P}+\left(\vec{K}_{H} \cdot \vec{E}_{H}\right) \vec{K}_{H}=0 .
$$

This set of equations completely describes the electromagnetic field inside the crystal [23]. This is a complex vector equation for each amplitude. $\vec{E}_{H}$, and must hold for the real and imaginary parts. and for each component of the vectors separately.

If only one reciprocal lattice point, $\vec{H}$. is near enough to the Ewald sphere to give any appreciable diffraction, we can neglect all field amplitudes except $\vec{E}_{0}$ and $\vec{E}_{H}$. Bragg's law defines the plane of incidence as that containing the wavevectors $\vec{K}_{0}$ and $\vec{K}_{H}$. The components of $\vec{E}_{0}$ and $\vec{E}_{H}$ may then be described by components parallel or perpendicular to this plane. The perpendicular component is called the $\sigma$ polarization. after the german word "senkrecht" for "perpendicular". The component in the diffraction plane is called the $\pi$-polarization.

For the $\sigma$-polarization state Equation 3.21 gives

$$
\left[k^{2}\left(1-\Gamma F_{0}\right)-\left(\overrightarrow{K_{0}} \cdot \overrightarrow{K_{0}}\right)\right] E_{0}^{\sigma}-k^{2} \Gamma F_{H} E_{H}^{\sigma}=0 .
$$

and

$$
-k^{2} \Gamma F_{H} E_{0}^{o}+\left[k^{2}\left(1-\Gamma F_{0}\right)-\left(\vec{K}_{H} \cdot \vec{K}_{H}\right)\right] E_{H}^{o}=0 .
$$


Nontrivial solutions to this pair of equations for the ratio $E_{H}^{\sigma} / E_{0}^{\sigma}$ requires that the determinant be zero. To generalize the relation for either polarization we let $P=1$. for the $\sigma$ polarization, and $P=\cos 2 \theta$, for the $\pi$ polarization. The following determinant must then equal zero for either value of $P$

$$
\left|\begin{array}{lr}
k^{2}\left(1-\Gamma \hat{H}_{0}\right)-\vec{h}_{0} \cdot \vec{h}_{0} & -k^{2} P\left[\vec{H}_{H}\right. \\
-k^{2} P \Gamma F_{H} & k^{2}\left(1-\Gamma F_{0}\right)-\vec{h}_{H} \cdot \vec{h}_{H}
\end{array}\right|
$$

Here $k$ is the vacuum wavevector. $\vec{K}_{0}$ and $\vec{h}_{H}$ are the wavevectors in the material. and $F_{0}, F_{H}, F_{H}$ are the structure factors for the $0, H$, and $\vec{H}$ reflections. The diagonal elements of the matrix represent the difference between the squares of the wavevectors $\vec{K}_{u}$ and $\vec{K}_{H}$ inside the crystal and the square of the vacuum wavevector. corrected for the average value of the dielectric constant. $\left(1-\Gamma F_{0}\right)$. As a result. the index of refraction for the incident and diffracted waves is different from the average index of refraction (Figure 3.2 ).

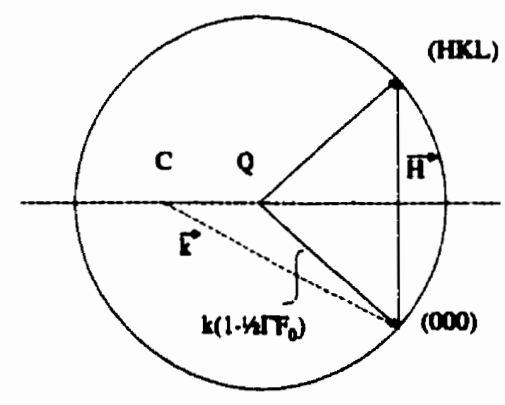

Figure 3.2: The Ewald sphere in reciprocal space. showing the wavevector corrected by the average index of refraction. The Laue point, $C$. represents the centre of the sphere in vacuum, while $Q$ is the centre in the real crystal.

Defining the difference parameters, $\xi_{0}$ and $\xi_{H}$, as

$$
2 k \xi_{0} \equiv \vec{h}_{0} \cdot \vec{K}_{0}-k^{2}\left(1-\Gamma F_{0}\right) \text {, }
$$


and

$$
2 k \xi_{H} \equiv \vec{K}_{H} \cdot \vec{K}_{H}-k^{2}\left(1-\Gamma F_{0}\right)
$$

the fundamental equation for the dispersion surface is found.

$$
\xi_{0} \xi_{H}=\frac{1}{4} k^{2} P^{2} \Gamma^{2} F_{H} F_{H}
$$

This is the fundamental result of the DTXRD [23]. It defines a constant energy surface which is the $\mathbf{X}$-ray analog of the Fermi surface in the electron band theory of solids. As a consequence of this relation. it is found that the DTXRD changes the concept of the Ewald sphere. In reciprocal space there is no longer a single Ewald sphere for a single monochromatic incident ray. The dispersion surface defined by Equation 3.27 defines the loci of all permitted Ewald spheres. Wavevectors from points on this surface to points in the reciprocal lattice represent wave solutions to Maxwell's equations.

In the usual case. Figure 3.1 , the distance from the centre. $C$. of the Ewald sphere to the origin and to a reciprocal lattice point, $(H K L)$ is equal to the vacuum wavevector. $k$. The dynamical framework takes into account the average index of refraction inside the material. $\vec{k}$ (inside) $=k\left(1-\frac{1}{2} \Gamma F_{0}\right)$. with the result that the centre of the Ewald sphere is now at point $Q$ as shown in Figure 3.2 .

The boundary conditions for the field and wave vectors can now be applied in order to find solutions for a particular experimental geometry. The direction cosines of the incident and diffracted beams with respect to the incident crystal surface are given by $\vec{n} \cdot \vec{s}_{0}=\gamma_{0}$ and $\vec{n} \cdot \vec{s}=\gamma_{H}$. where $\vec{n}$ is an inward unit vector and $\vec{s}_{0}$ and $\vec{s}$ are unit vectors in the incident and diffracted beam directions.

The dispersion parameters are found to be [23]

$$
\xi_{0}=\frac{1}{2} k|P \| b|^{1 / 2} \Gamma\left[F_{H} F_{H}\right]^{1 / 2}\left[\eta \pm\left(\eta^{2}+b /|b|\right)^{1 / 2}\right]
$$

and

$$
\xi_{H}=\frac{1}{2} k|P|\left(\Gamma /|b|^{1 / 2}\right)\left[F_{H} F_{H}\right]^{1 / 2}\left[\eta \pm\left(\eta^{2}+b /|b|\right)^{1 / 2}\right]^{-1},
$$

where $b \equiv \gamma_{0} / \gamma_{H}$ and

$$
\eta \equiv \frac{\left[b \Delta \theta \sin 2 \theta_{B}+\frac{1}{2} \Gamma F_{0}(1-b)\right]}{\Gamma|P \| b|^{1 / 2}\left[F_{H} F_{H}\right]^{1 / 2}}
$$




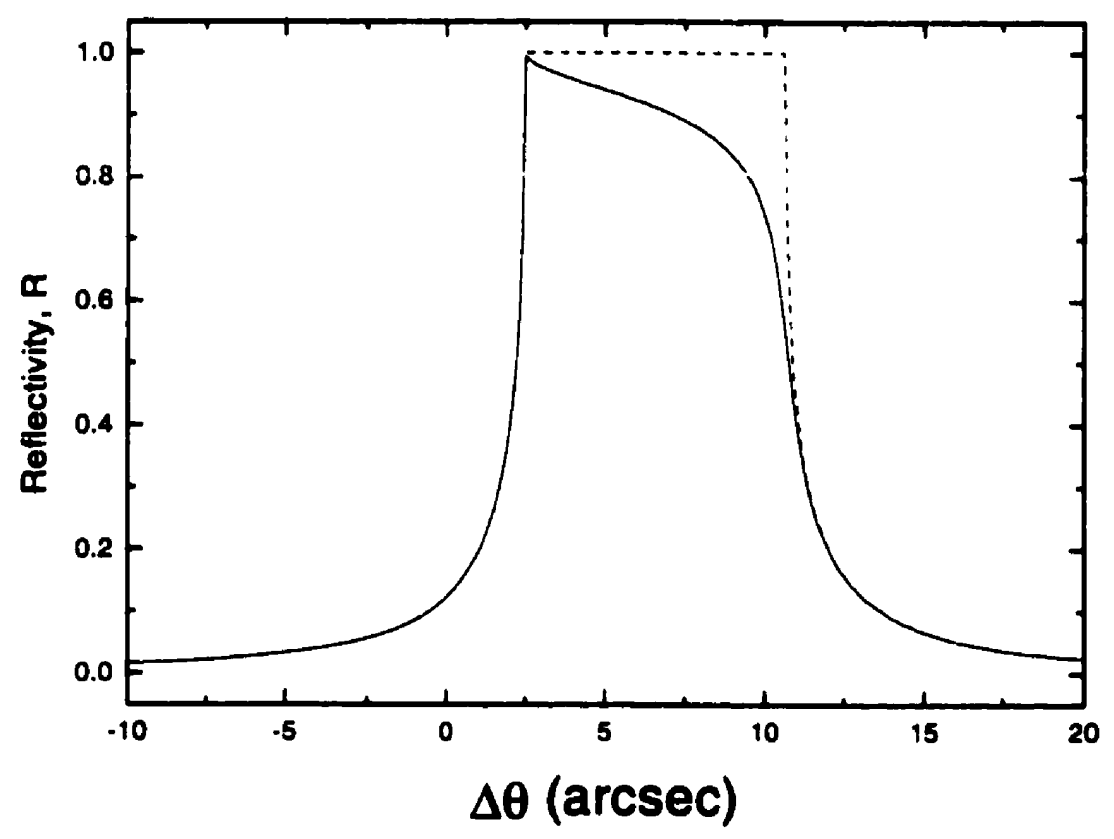

Figure 3.3: Shape of the (004) symmetric Bragg reflection for a GaAs crystal. The upper dotted curve is for zero absorption (Darwin curve) and the lower curve includes the absorption (Darwin-Prins curve).

To determine the ratio of the diffracted and incident electric field amplitudes for polarization $P$. from Equations 3.22 and 3.23. from the analogous relations for the $\pi$-polarization. and from the definitions of the difference parameters.

$$
\frac{E_{H}}{E_{0}}=\frac{-2 \xi_{0}}{k P \Gamma F_{A}}=\frac{-k P \Gamma F_{H}}{2 \xi_{H}} .
$$

In this thesis we consider the symmetric Bragg case, in which $b=-1$, where the diffracted beam exits from the same face of the crystal that the incident beam enters and at the same angle with respect to the surface. The form of the reflectivity curve in this geometry was derived by Darwin [28]. In this case the incident amplitudes inside and outside the crystal are the same, and the outside diffracted amplitude is equivalent to the inside diffracted field at the surface. Thus the diffracted beam is a 
reflection. Multiplying the two expressions in Equation 3.31, we find

$$
\left(\frac{E_{H}}{E_{0}}\right)^{2}=\frac{\xi_{0} F_{H}}{\xi_{H} F_{R}}=|b|\left(\eta \pm\left(\eta^{2}-1\right)^{1 / 2}\right)^{2} \frac{F_{H}}{F_{R}} .
$$

The magnitude of this expression is the reflectivity which can be measured experimentally,

$$
R=\frac{\left|F_{H}\right|}{\left|F_{\dot{H}}\right|}\left|b \| \eta \pm .\left(\eta^{2}-1\right)^{1 / 2}\right|^{2} .
$$

The sign to be chosen is opposite in sign to $\operatorname{Re}(\eta)$, and is chosen to keep $R<1$. When the crystal absorption is taken into account [29], a plot of the reflectivity versus the deviation from the Bragg angle. $\Delta \theta=\theta-\theta_{B}$. is known as the Darwin-Prins curve, or crystal rocking curve.

The reflectivity curve for a CiaAs crystal with $(H K L)=(004)$ is shown in Figure 3.3 for C $u-K_{\alpha_{1}} X$-rays $(\lambda=1.54061 \mathrm{~A})$. The effect of the crystal absorption is seen in the figure, from which it is apparent that the absorption is more pronounced at the high angle side. This is a manifestation of the standing waves due to the interference field which is produced in the crystal. Having now reviewed the fundamental results of the DTXRD we consider the standing wave phenomenon in the next section.

\subsection{X-Ray Standing Waves}

The interference of the incident and diffracted waves in a perfect crystal produces a standing wave pattern as a consequence of the dynamical nature of the interaction and was discussed in the thorough review by Batterman and Cole [23]. Following the experimental observation [30] of fluorescence scattering by foreign atoms (As) in a silicon crystal associated with the standing wave field, the technique has found widespread application in many areas of surface science. The use of synchrotron radiation has made measuring times reasonable, reduced the effects of the dispersiveness of crystal arrangements, and allowed a wide range of $X$-Ray energies to be employed. This section reviews the principles of the XSW technique, particularly those aspects which enable the measurement of $\delta$-layers embedded in epitaxial semiconductors. 


\subsubsection{Principles of the XSW Technique}

An outstanding XSW review by Zegenhagen [31] covers the important theoretical and practical aspects of the technique. especially for the evaluation of adsorbate species on crystal surfaces. A feature of XSW for the study of adsorbates or buried impurities in crystals is that the technique is element-sperific, thus atomic distributions may he detected individually, and positional information may be obtained with an accuracy of $0.01 \AA$ under favourable conditions. However, it must be verified that the measured signal is actually originating from the atoms under consideration.

The formalism for XSW structural analysis was developed by Hertel $t$ al.[32]. They recognized that analyzing a distribution of atoms with XSW and diffraction vector $\vec{H}$ is equivalent to determining the amplitude. $F^{H}$. and phase. $P^{H}$. of the $\vec{H}$ th Fourier component of the atomic distribution function.

$X$-ray standing waves are generated by the superposition of two coherently related travelling waves. We assume that the waves are planar and linearly polarized and that the electric field vectors of both waves are parallel. We will restrict our attention to the $\sigma$-polarization which is most commonly used. Representing the incident and reftected waves by their complex amplitudes. $\mathcal{E}_{0}$ and $\mathcal{E}_{H}$. respectively, we have [31]

$$
\mathcal{E}_{0}=E_{0} \exp \left[2 \pi i\left(\nu_{0} t-\overrightarrow{h_{0}} \cdot \vec{r}\right)\right] .
$$

and

$$
\mathcal{E}_{H}=E_{H} \exp \left[2 \pi i\left(\nu_{H} t-\vec{\kappa}_{H} \cdot \vec{r}\right)\right] .
$$

where $\nu_{0}=\nu_{H}=\nu$ is the frequency of the radiation. and $\vec{r}$ is a coordinate vector relative to an arbitrary origin. Bragg's law relates the incident, $\vec{K}_{0}$, and diffracted. $\vec{K}_{H}$, wavevectors as $\vec{K}_{H}=\vec{K}_{0}+\vec{H}$, in terms of the diffraction vector. $\vec{H}$, where $\left|\vec{K}_{H}\right|=\left|\vec{K}_{0}\right|$. Assuming $\mathcal{E}_{0}$ and $\mathcal{E}_{H}$ are coherent, the (complex) amplitudes $E_{0}$ and $E_{H}$ are related via the phase factor $\alpha$ as

$$
E_{H}=\sqrt{R} E_{0} \exp (i \alpha) .
$$

Here $R$ and $\alpha$ denote the intensity and the phase of the reflected wave relative to the incident wave, respectively, where the reflectivity is determined from the DTXRD (Section 3.1). 
Superposition of the two waves yields an expression [31] for the intensity of the interference field in terms of the total field,

$$
\mathcal{E}=\mathcal{E}_{H}+\mathcal{E}_{0}
$$

as

$$
\mathcal{E} \mathcal{E}^{*} /\left\|E_{0}\right\|^{2}=I=1+R+2 \sqrt{R} \cos (\alpha-2 \pi \vec{H} \cdot \vec{r}) .
$$

It is convenient to discuss the reflectivity in terms of the quantitity " (c.f. Equation 3.30) for generality. Note that low values of $\eta$ correspond to high values of $\Delta \theta$ and vice-versa. As the incident $X$-ray energy moves from the high $\eta$ side of the Bragg peak to the low $\eta$ side, the phase of the wavefield changes continuously by $\pi$. i.e..

$$
\begin{gathered}
\alpha=\phi . \quad \text { for } \operatorname{Re}\left(E_{H} / E_{0}\right)>0 . \\
\alpha=0+\pi . \quad \text { for } \operatorname{Re}\left(E_{H} / E_{0}\right)<0 .
\end{gathered}
$$

where

$$
\phi=\tan ^{-1}\left[\frac{\operatorname{Im}\left(E_{H} / E_{0}\right)}{\operatorname{Re}\left(E_{H} / E_{0}\right)}\right] .
$$

These expressions describe the spatial modulation of the wavefield [31]. A standing wave is produced in the crystal with maxima and minima lying on planes in the direction normal to $\vec{H}$. At the high $\eta$ side of the Bragg reflection the nodes of the XSW lie on the substrate planes. As the phase changes in the region of the Bragg peak the wavefield moves continuously until the antinodes lie on the substrate planes at the low $\eta$ side.

Notice that Equation 3.38 does not depend on time. The intensity of the wavefield is spatially modulated since $\vec{H} \cdot \vec{r}$ is a dimensionless, real number. In the direction of $\vec{H}$ the intensity modulation is maximum and zero in the direction normal to $\vec{H}$. Minima (nodes) and maxima (antinodes) of the wavefield lie on planes. Thus the superposition of the two coherent plane waves $\mathcal{E}_{0}$ and $\mathcal{E}_{H}$ creates a planar interference field in the crystal.

The detection of the XSW intensity at the location of a particular atomic species requires the measurement of some property which is proportional to that intensity. 
In the X-Ray energy range the important inelastic X-Ray scattering process is photoabsorption. Here a photon with energy $E_{\gamma}$ is completely absorbed and

$$
E_{\gamma}=E_{f}-E_{i}
$$

where $E_{f}$ and $E_{i}$ are the binding energies of the final (excited) and initial states of the electron. respectively. The electron is excited into empty states above the Fermi level. in the same manner as occurs in the XAFS technique (Chapter 4). It is assumed that the scattering probability and thus the scattered intensity, $I_{s c}$, is a linear function of the X-Ray intensity at the position of the scatterer [31].

From Figure 3.4 it is apparent that for a given plane spacing, $d_{s}$, moving the standing wave in a controlled way allows a measurement of the distance between $A$ and $\mathrm{B}$ along $z$, by determining how much the wavefield must shift in order to observe maximum scattering from $A$ or B. Figure 3.4 also shows that this distance can only be determined modulo $d_{s}$.

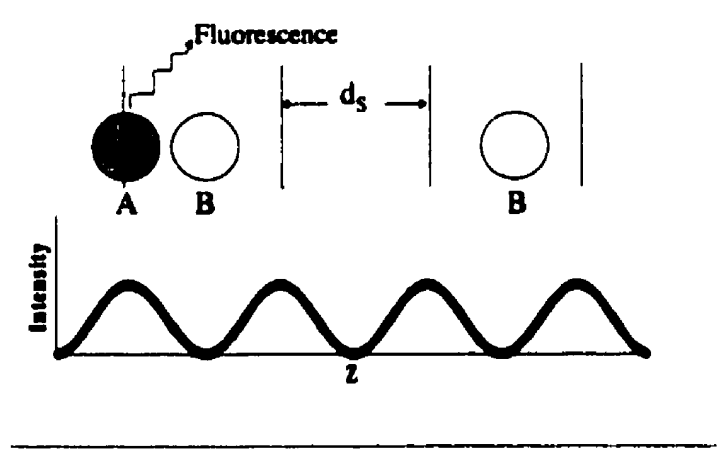

Figure 3.4: Schematic of the XSW technique. Maximum fluorescence from a particular atomic species is observed when the antinodal planes pass through the atoms. The fluorescence emission is characteristic of the absorbing atoms.

The wavefield given by Equation 3.38 has a spacing, $d_{s}$, determined by the relationship between the propagation vectors $\vec{K}_{0}$ and $\vec{K}_{H}$. Denoting the $z$-coordinate 
parallel to $\vec{H}$ as $z_{H}$, where $H=|\vec{H}|$, we have [31]

$$
\vec{H} \cdot \vec{r}=H z H .
$$

The period of the cosine function in Equation 3.38 is $H d_{s}=1$. thus the spacing, $d_{s}$, between the wavefield maxima is

$$
d_{s}=H^{-1}=\left|\vec{K}_{H}-\vec{h}_{0}\right|^{-1} .
$$

From Bragg`s law.

$$
d_{s}=H^{-1}=\frac{1}{2} \lambda(\sin \theta)^{-1} .
$$

and so the smallest spacing, $d_{s, m i n}$, of the wavefield is

$$
d_{s, \min }=\frac{\lambda}{2} . \text { for } \theta \approx 90^{\circ} .
$$

Similarly, the wavefield spacing increases as $\theta$ approaches zero.

$$
d_{s, \max } \rightarrow \infty . \text { for } \theta \rightarrow 0
$$

By adjusting $\vec{H}$ or $\theta$ it is thus possible to adjust the wavefield spacing to the scale of a given structural problem.

Of the experimental geometries producing standing waves in crystals the Bragg case is the most useful and the only one in the present discussion. The total reflection geometry near $\theta=0$ can also be formally considered as a 0 th order Bragg reflection and is discussed in Section 4.3.3. Scanning through the Bragg condition in a crystal is accomplished by varying the parameter $\eta$. A traditional setup involves a rocking curve measurement in which the angle of incidence, $\theta$, of the $X$-Ray beam is varied throughout the angular range of the Bragg reflection. This geometry will be discussed in detail in connection with the experimental HRXRD studies described in Section 6.5.

It has been noted [33], however, that for the Bragg case close to $\theta=90^{\circ}$ the variable parameter of Equation 3.30 may be rewritten

$$
\eta=\frac{\left(-2 \Delta E / E+\Gamma F_{0}\right)}{\Gamma\left[F_{H} F_{\dot{H}}\right]^{1 / 2}} .
$$


Passing through the range of the Bragg reflection, the phase, $\alpha$, changes by $\pi$ and the XSW field moves half a diffraction plane spacing. This can be achieved by tuning $\eta$ either using the angle, $\theta$, or the energy, $E$. In the case of noticeable absorption the phase change of exactly $\pi$ can only be achieved by extending the angular range far beyond the width of the Bragg reflection region.

The orientation of the wavefield pattern is determined [31] by the diffraction vector. $\vec{H}$. When $\vec{H}$ is oriented normal to the crystal surface the wavefield planes are oriented parallel to the surface. Other orientations can also be employed. provided the diffracted wave appears at the same crystal face as the incident wave. Several measurements using different reflections can be useful in realizing a complete structural analysis. Figure 3.5 shows the reflectivity. $R$, the phase. $a$. and the wavefield intensity. $I$. for several positions $\vec{r}$ relative to the $(004)$ planes of a $(001)$-oriented GaAs crystal in the backreflection geometry.

The total reflection range of a symmetric Bragg reflection of $\sigma$-polarized $X$-Rays has a width

$$
W_{\theta}=\frac{2 \Gamma\left(F_{H} F_{H}\right)^{1 / 2}}{\sin \left(2 \theta_{B}\right)} .
$$

This result is derived from Equation 3.30. and corresponds to a range of $\eta^{\prime}=-1$ to $\eta^{\prime}=+1$, where $\eta^{\prime}=\eta-F_{0} /\left(F_{H} F_{A}\right)^{1 / 2}$. For example. the $\operatorname{CaAs}(004)$ rocking curve shown in Figure 3.3 for the case of no absorption has $W_{\theta}=8.2$ arcsec. As the incidence angle is increased $\sin \left(2 \theta_{B}\right)$ becomes very small and the angular range increases steeply [33]. In the back-reflection mode $\left(\theta_{B} \approx 90^{\circ}\right)$. Equation 3.30 no longer applies, nor does Equation 3.48. If the rocking curve is scanned in energy, however, as suggested by Equation $3.4 \bar{i}$, then the rocking curve width is increased strongly. In energy, the back-reflection rocking curve has a total reflection width given by $W_{E}=E \Gamma\left(F_{H} F_{\bar{H}}\right)^{1 / 2}$. Note that the back-reflection geometry restricts the choice of wavelengths to those for which $\lambda=2 d_{s}$.

The experimental details describing the use of back-reflection XSW will be presented in Chapter 6.6. In the following section the methods for interpreting XSW data are described. 


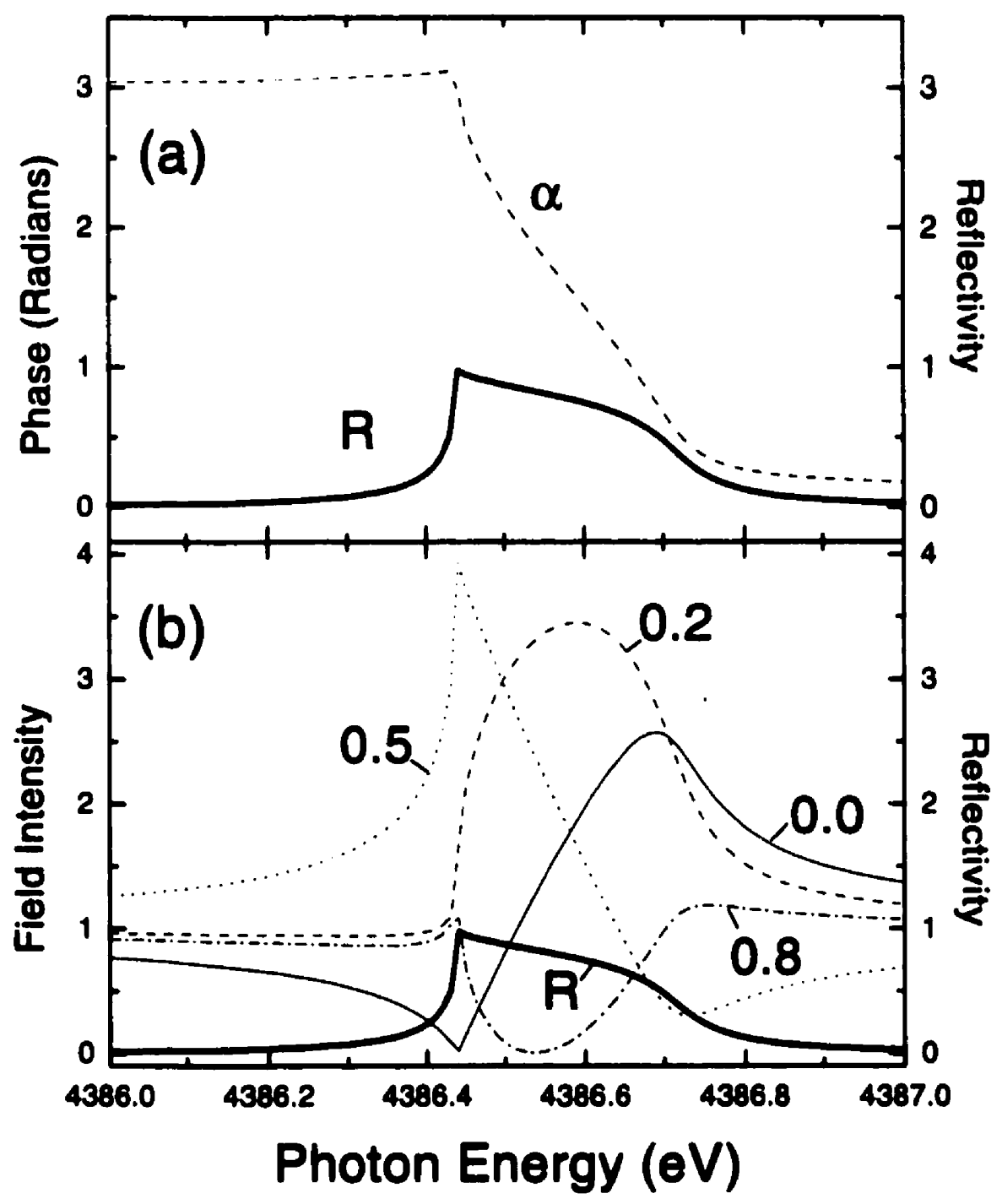

Figure 3.5: (a) Reflectivity, $R$, and phase, $a$, of the symmetric GaAs(004) backreflection. (b) Reflectivity and XSW intensities for different values of $H z_{H}$ within the GaAs unit cell, as indicated. 


\subsubsection{XSW Analysis}

As discussed in Section 3.2.1 the intensity of the XSW wavefield at the location of a given atom is given by the expression

$$
I=1+R+2 \sqrt{R} \cos (\alpha-2 \pi \vec{H} \cdot \vec{r}) .
$$

In practice, the measurement is made by monitoring an emission channel which is proportional to this intensity. Fluorescence emission has been used exclusively in this work, although the measurement of photoelectrons or Auger electrons are also common. In these cases, XSW is complemented by X-Ray photoelectron spectroscopy (XPS) and Auger electron spectroscopy (AES), respectively.

The measurement of the atomic fluorescence as the XSW emission channel requires the use of the dipole approximation [31]. In practice, the quadropole contribution to the absorption cross-section is small and the dipole approximation is adequate. The use of the fluorescence channel combines the XSW technique with the features of energy-dispersive $X$-Ray spectroscopy (EDX).

An XSW measurement samples the fluorescence from all of the atoms of the species of interest within the crystal [31]. For $N$ atoms. with positions denoted by $\vec{r}_{i}$, the scattered yield is

$$
Y^{-H}=1+R+2 \sqrt{R} N^{-1} \sum_{i=1}^{N} \cos \left(\alpha-2 \pi \vec{H} \cdot \vec{r}_{i}\right) .
$$

This can be expressed in terms of a distribution function $G(\vec{r})$, where

$$
Y^{\cdot H}=1+R+2 \sqrt{R} \int_{V^{\prime}} C(\vec{r}) \cos (\alpha-2 \pi \vec{H} \cdot \vec{r}) d \vec{r} .
$$

and $\int_{V} G(\vec{r}) d \vec{r}=1$. Here the integral is taken over the volume. $l$. containing the $X$-Ray interference field.

The component of $\vec{r}_{i}$ parallel to $\vec{H}$ is $z_{i}^{H}$, where

$$
\vec{H} \cdot \vec{r}_{i}=|\vec{H}| z_{i}^{H}=P_{i}^{H} .
$$

The position of the atom of interest with respect to the diffraction planes of the substrate is given by $P_{i}^{H}$, where $0 \leq P_{i}^{H}<1$. Thus $P_{i}^{H}$ measures the position of a 
given atomic species relative to a diffraction plane of the set $\vec{H}$, normalized by the plane spacing, $d_{s}$. Note that $P_{i}^{H}$ is actually determined modulo- $d_{s}$ so that values of e.g. 0.15 and 1.15 are equivalent. With this notation. Equation 3.51 may be rewritten

$$
Y^{H}=1+R+2 \sqrt{R} F^{H} \cos \left(\alpha-2 \pi P^{H}\right) .
$$

The parameters $P^{H}$ and $F^{H}$ are called the coherent position and coherent fraction. respectively [31]. If the atomic species under consideration occupy $n$ positions $\left(P_{i}^{H}\right)$ relative to the diffraction planes, then $P^{H}$ and $F^{H}$ may be written [32]

$$
F^{H}=A_{s}^{H} \equiv\left(G_{H}^{c}{ }^{2}+G_{H}^{s}\right)^{1 / 2}
$$

and

$$
P^{H}=\frac{1}{2 \pi} \tan ^{-1}\left(\frac{G_{H}^{s}}{G_{H}^{c}}\right)+\left\{\begin{array}{cc}
0.5 & \text { if } G_{H}^{\prime c}<0 \\
0 & \text { otherwise }
\end{array} .\right.
$$

where the cosine term. $C_{H}^{c}$, is

$$
C_{H}^{c}=\sum_{i=1}^{n} c_{i} \cos \left(2 \pi P_{i}^{H}\right)
$$

and the sine term. $C_{H}^{3}$, is

$$
C_{H}^{s}=\sum_{i=1}^{n} c_{i} \sin \left(2 \pi P_{i}^{H}\right)
$$

The coefficients in these expressions satisfy the normalization $\sum_{i=1}^{n} c_{i}=1$. where $c_{i}$ denotes the relative population of the position $P_{i}^{H}$.

Thermal vibrations may be taken into account by convolution of $G(\vec{r})$ with a normalized Gaussian distribution:

$$
G_{G}(\vec{r})=\frac{1}{\sqrt{2 \pi} \sigma_{H}} \exp \left[-\frac{z_{R}^{2}}{2 \sigma_{H}^{2}}\right]
$$

where

$$
\sigma_{H}^{2}=<u_{H}^{2}>H^{2} .
$$

Here $z_{R} \equiv \Delta d / d_{H}$ where $\Delta d$ is the displacement of the atom from the diffraction planes. The mean-square vibrational amplitude of the atoms in the direction of $\vec{H}$ is $<u_{H}^{2}>$. 
Now, the coherent fraction including the thermal disorder is [31]

$$
F^{H}=A_{s}^{H} D^{H}
$$

where the Debye-Waller factor is $D^{H}=\epsilon^{-M}$ and $M=2 \pi^{2}<u_{H}^{2}>H^{2}$. Note that the coherent position is unaffected by the thermal disorder. Very careful diffrartion studies [34] have established that the room temperature Debye-Waller factor for the GaAs (004)-reflection has a value of $\sim 0.9$.

In this section the method of XSW analysis has been presented in a manner which permits the interpretation of the experimental data for layers of In.As embedded in GaAs, as will be discussed. In the following section the D'TXRD framework described in an earlier section is extended to allow the interpretation of diffraction data from layered semiconductor heterostructures.

\subsection{Simulation of X-Ray Rocking Curves}

As mentioned in the introduction to this chapter, the analysis of high-resolution X-Ray diffraction data relies upon the solution to the so-called Takagi-Taupin equations. A treatment of this subject is now presented which leads to the elegant form of solution derived by Bartels et al. [17].

The X-Ray diffraction theory for crystals with a strain gradient perpendicular to the surface was developed by Takagi [25] and independently by Taupin [26] shortly thereafter. According to Taupin, the system of equations may be written as

$$
i \frac{\lambda \gamma_{H}}{\pi} \frac{d D_{H}}{d z}=\Psi_{0} D_{H}+\Psi_{H} D_{0}-\alpha_{H} D_{H} \text {. }
$$

and

$$
i \frac{\lambda \gamma_{0}}{\pi} \frac{d D_{0}}{d z}=\Psi_{0} D_{0}+\Psi_{H} D_{H}
$$

Here $D_{0}$ and $D_{H}$ are the amplitudes of the incident and diffracted waves at the depth $z$ in the crystal. The direction cosines are $\gamma_{0}$ and $\gamma_{H}$, and $\lambda$ is the $X$-Ray wavelength in vacuum. The quantities $\Psi_{0}$ and $\Psi_{H}$ are related to the structure factors of the material at the depth $z$ as $\Psi_{0}=-\left(\lambda^{2} r_{e} / \pi V\right) F_{0}$ and $\Psi_{H}=-\left(\lambda^{2} r_{e} / \pi V\right) F_{H}$, where $r_{e}$ 
is the classical electron radius as defined in section 3.1. Taupin defined

$$
\alpha_{H}=\lambda^{2}\left(\frac{1}{d_{H}^{2}}-\frac{2 \sin \theta_{B}}{\lambda d_{H}}\right)
$$

where $d_{H}$ is the diffraction plane spacing at the depth $z$. Denoting the amplitude ratiu by $X^{\prime}=D_{H} / D_{0}$, the system of equations nay be cumbined tu give [35]

$$
\frac{d . X}{d z}=\frac{i \pi}{\lambda \sin \theta_{B}}\left[\Psi_{H} \cdot X^{2}+2 \Psi_{0}-\alpha_{H}(z) X+\Psi_{H}\right] .
$$

Here the quantity $\alpha(z)$ takes into account the deviation from the exact Bragg condition.

$$
\alpha_{H}(z)=\alpha_{H}(0)+J \alpha_{H}(z) .
$$

where $\alpha_{H}(0)$ is the deviation of the incident angle from the substrate Bragg angle. and $د \alpha_{H}(z)$ is the additional deviation due to the presence of impurities in the crystal (or epitaxial heterolayers). With this formulation the equations were solved numerically. using the Runge-Kutta method [35]. In this way Burgeat and Taupin were able to determine the diffusion coefficient of boron in silicon from experimental XRD profiles.

In the SFU MOVPE laboratory a commercial software package is used for the simulation of rocking curves from III- $\mathrm{V}$ heterostructures. ${ }^{1}$ The program employs a solution to the Takagi-Taupin equations derived by Halliwell et al. [36] and Hill et al. [3i]. A shortcoming of the algorithm describerl in these articles is that the relationship with the Darwin-Prins formula (Equation 3.33) for an infinitely thick. absorbing, perfect crystal is not transparent. Moreover. details of the method are omitted from these articles, perhaps for proprietary reasons.

Bartels et al. [17] developed a recursive method of solution based on Taupin's single differential equation for the Bragg case,

$$
-i \frac{d X}{d T}=X^{2}-2 \eta X+1
$$

where

$$
X=\left(\frac{F_{H}}{F_{H}}\right)^{1 / 2}\left|\frac{\gamma_{H}}{\gamma_{0}}\right|^{1 / 2} \frac{D_{H}}{D_{0}} .
$$

\footnotetext{
'RADS: Rocking Curve Analysis by Dynamical Simulation, BEDE SCIENTIFIC, UK
} 
and

$$
T=\frac{\pi P \Gamma\left(F_{H} F_{A}\right)^{1 / 2} t}{\lambda\left|\gamma_{0} \gamma_{H}\right|^{1 / 2}}
$$

The quantity $\eta$ and other values are defined as in Section 3.1. The layer thickness. $t$. and the structure factor. $F_{H}$, are used to evaluate the quantity. $T$. The differential erylution (3,66) can then he solver for layers of constant $\eta$ and arbitrary thickness [17]. Recursion relations are used to describe the amplitude ratio at the bottom of a given layer, $x_{0}$. to the amplitude ratio at the top, $x_{t}$.

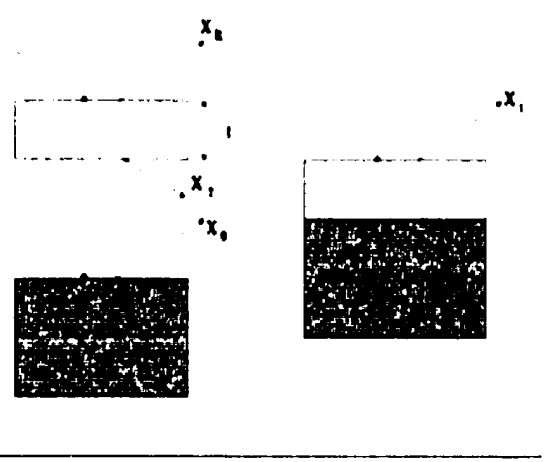

Figure 3.6: The recursion method involves adding a laver of thickness $t$ to the substrate so that the reflected amplitude at the surface changes from $x_{0}$ to $x_{t}$.

Now.

$$
X_{t}=\eta+\left(\eta^{2}-1\right)^{1 / 2}\left[\frac{\left(S_{1}+S_{2}\right)}{\left(S_{1}-S_{2}\right)}\right]
$$

where

$$
S_{1}=\left[X_{0}-\eta+\left(\eta^{2}-1\right)^{1 / 2}\right] \exp \left[-i T\left(\eta^{2}-1\right)^{1 / 2}\right] .
$$

and

$$
S_{2}=\left[X_{0}-\eta-\left(\eta^{2}-1\right)^{1 / 2}\right] \exp \left[i T\left(\eta^{2}-1\right)^{1 / 2}\right] .
$$

These relations describe the general solution for the dynamical reflection of an epitaxial layer of arbitrary thickness [17], and allow the calculation of rocking curves of complicated layer structures. For an infinitely thick crystal the result reduces to the Darwin-Prins formula (Equation 3.33). In the calculation the reflected amplitude 
deep within the substrate is taken to be zero. Figure 3.3 illustrates the situation for a single layer and a substrate. In the figure $X_{R}$ and $X_{T}$ are the reflected and transmitted amplitude ratios for the epitaxial layer.

To demonstrate the use of this method. Figure 3.i shows the results obtained using the recursion relations for structures of interest to this thesis. In the bottom part. a single ML of InAs deposited on $\mathrm{GaAs}(001)$ and buried by $100 \mathrm{~A}$ of GaAs is considered using the GaAs(004) symmetric reflection. with $\sigma$-polarized $\mathrm{C}^{\prime} \mathrm{u}-\mathrm{K}_{\mathrm{u}_{1}} \mathrm{X}$-Rays. The top part shows a superlattice structure, in which the (1 ML InAs/ $100 \mathrm{~A}$ GaAs) growth sequence is repeated 35 times. It is apparent that the present method of simulation agrees very well with the results obtained using the commercial software. The small discrepancies may arise because the commercial package neglects the volume change (and hence the change in susceptibility) which occurs in strained epitaxy, and because of small differences between the material parameters used in the two programs.

This chapter has described the principles of the diffraction-based techniques employed in this work. In the following chapter the XAFS technique will be discussed. These long-range order and short-range order techniques complement one another in the measurements of semiconductor heterostructures studied in this thesis. 


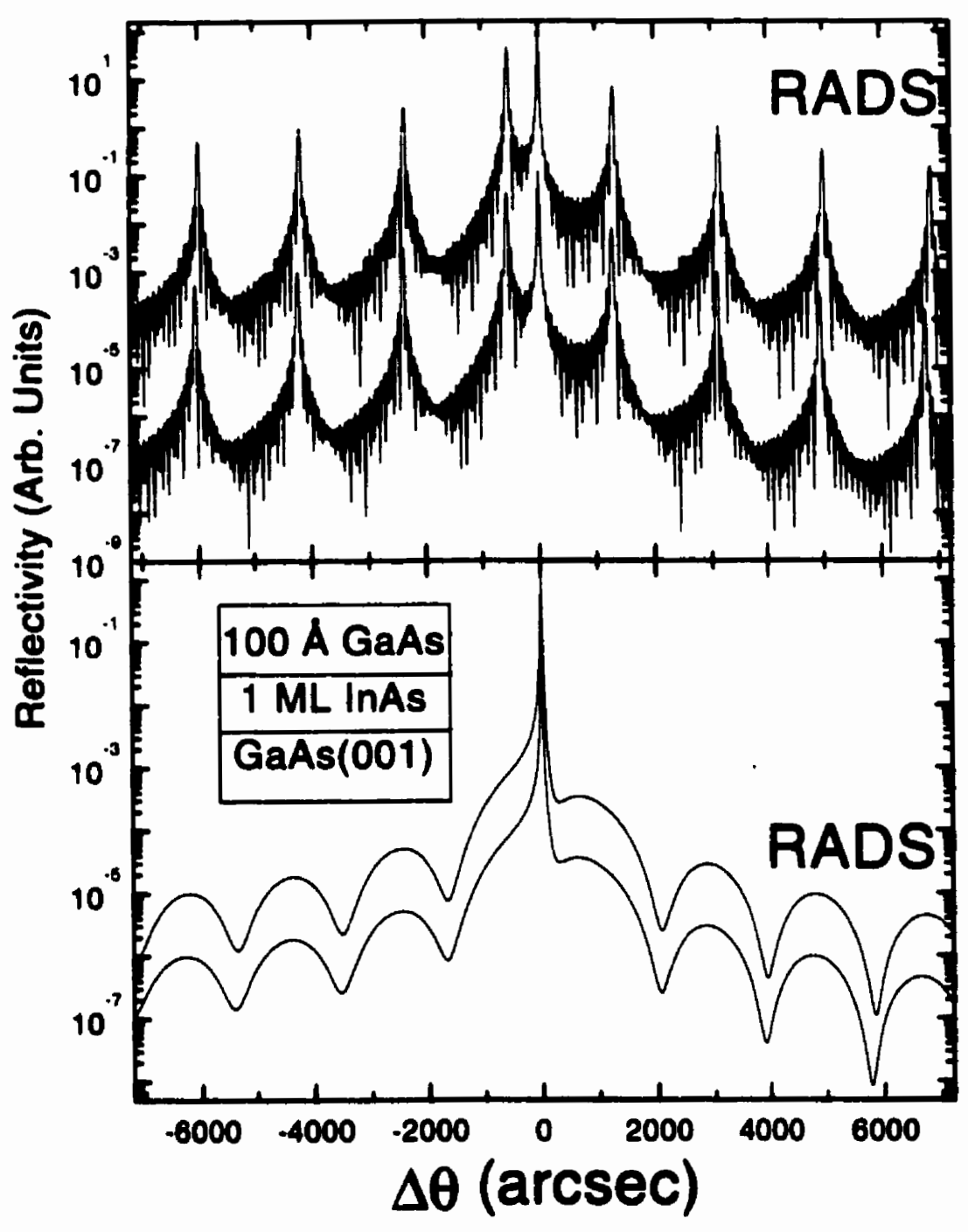

Figure 3.i: Simulated X-Ray rocking curves for a GaAs(001)/1 ML InAs/100 A GaAs structure (bottom) and for a $35 \times(1 \mathrm{ML} \operatorname{InAs} / 100 \AA \mathrm{GaAs})$ superlattice on $\mathrm{GaAs}(001)$ (top). The simulations were carried out in the neighbourhood of the (004) substrate reflection in the $\sigma$-polarization. The top curve in each part illustrates the results obtained using the RADS package. The curves were offset for clarity. 


\section{Chapter 4}

\section{X-Ray Absorption Fine Structure}

In this chapter the principles of the XAFS technique will be described. First a general, conceptual description of the phenomenon will be presented. The mathematical expression for the process in the extended regime will be given in a form which includes the polarization dependence, of interest in the measurement of oriented (semiconductor) samples. Finally the glancing incidence measurement geometry and the calculation of reflectivity curves for lavered samples will be discussed.

\subsection{Principles of XAFS}

XAFS refers to the structure which appears in the absorption coefficient of atoms in condensed matter during the absorption of $\mathrm{X}$-Ray photons from a synchrotron source. for example. This consists of two regimes. In the neighbourhood of the absorption edge, the X-Ray absorption near edge structure (XANES) is observed. For increasing photon energies, the extended X-Ray absorption fine structure (EXAFS) appears. It is the latter which is primarily of interest to this thesis, although the "extended" qualifier is often omitted. Excellent reviews are available in the literature [38], including a review of very recent developments by Crozier [39].

$\mathrm{X}$-Ray photons are absorbed by atoms when the X-Rays are sufficiently energetic to free a bound electron in the atom [40]. When the electrons are in the most tightly bound $(n=1)$ shell, the edge is called the K-edge. For the next most tightly bound 
electrons the edge is known as the L-edge, and so on. For photon energies up to about $40 \mathrm{keV}$ the incident photon is completely absorbed, transferring its energy to excite a photoelectron and leaving behind a core hole. In the measurements described in this thesis. the hole is subsequently filled by an electron in a higher shell with the emission of a fluorescence photon. By monitoring the fluorescence emission of the absorbing species it is possible to measure quantitatively the absorption coefficient of these atoms.

If all of the absorbed photon's energy is used to excite the core electron then the kinetic energy of the photoelectron is given by the difference between the incident photon energy and the binding energy of the electron in the atom [40]. From the deBroglie relation the momentum of the outgoing photoelectron is $p=h / \lambda$, in terms of the wavelength, $\lambda$ and Planck's constant. $h$. The momentum is given by

$$
\frac{p^{2}}{2 m}=h \nu-E_{0}
$$

where $\nu$ is the frequency of the X-Ray photon and $E_{0}$ is the binding energy of the photoelectron.

XAFS does not occur for isolated atoms. In such a situation the absorption is a smooth function of energy above the absorption edge. For an absorbing atom surrounded by some arrangement of neighbouring atoms, the outgoing photoelectron wave is scattered by those neighbours. Figure 4.1 shows such an arrangement for the first coordination shell of an atom in the zincblende lattice. The final state consists of the superposition of the outgoing and scattered waves.

Quantum mechanically the absorption process is described by Fermi's Golden Rule [41]. The transition rate depends on the dipole operator and may be written

$$
W_{i \rightarrow f}=\left(\frac{2 \pi}{\hbar}\right)\left(\frac{e \mathcal{E}_{0}}{2}\right)^{2}|<f| z|i>|^{2} \rho\left(E_{f}\right) .
$$

Here $\rho\left(E_{f}\right)$ is the density of final states. the initial $K$-shell core state is $\mid i>$ and the final state of the electron excited to the escaping photoelectron is $|f\rangle$. Their respective energies are $E_{i}$ and $E_{f}=E_{i}+\hbar \omega$, where $\hbar \omega$ is the photon energy [40]. The electric field is denoted by $\mathcal{E}_{0}$ and is oriented along the $z$-direction. 


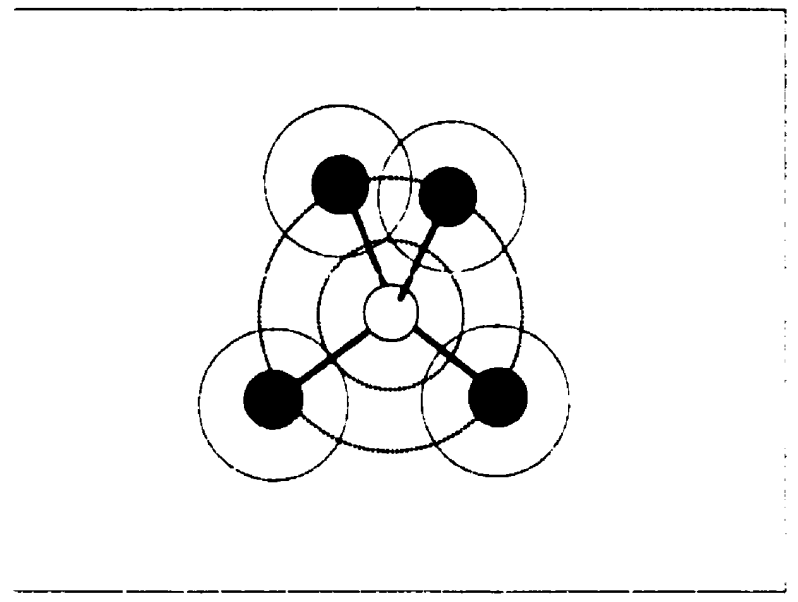

Figure 4.1: The propagation of the spherical photoelectron wave (heavy dashed lines) excited from an atom in the zincblende lattice. Backscattering from the atoms in the first coordination shell (light lines). and higher shells to a lesser extent. leads to the oscillatory behaviour in the absorption coefficient.

The backscattered waves affect the outgoing wave at the centre of the absorbing atom by contributing a characteristic phase. The total amplitude of the electron wave function will be enhanced or reduced. causing a corresponding change in the probability of X-Ray absorption [40]. The oscillations, or fine structure above the absorption edge thus exhibits peaks when the backscattered wave is in phase with the outgoing wave. and valleys when the two are out of phase. These characteristics are apparent in Figure 4.2 which shows the K-edge XAFS for a GaAs powder sample.

The variation of the phase with the photoelectron energy depends on the distance between the absorber and the backscatterer. The variation in the amplitude of the backscattering as a function of energy depends on the type of backscatterer. Thus. in Figure 4.2 the XAFS contains element-specific information on the local environment of the $\mathrm{Ga}$ absorbing atoms. In the following section a mathematical expression is provided to permit the analysis of experimental data. 


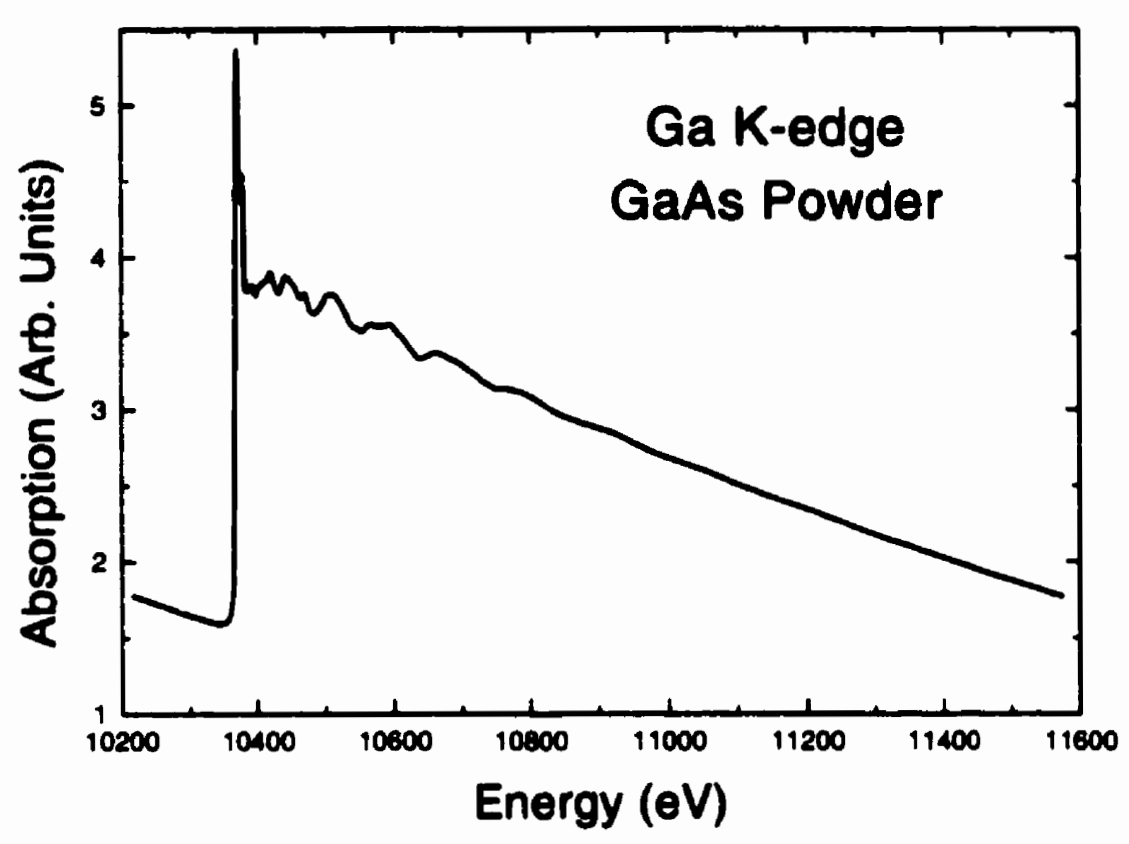

Figure 4.2: Ga K-edge XAFS from a Ga.ts powder sample. The data were obtained in transmission at beamline X23A2 at the NSLS.

\subsection{The EXAFS Expression}

When the energy of incident photons is increased through the absorption edge, the XAFS, $\gamma(E)$ appears as an additional term in the absorption,

$$
\mu(E)=\mu_{0}(E)(1+\gamma(E))
$$

where $\mu_{0}(E)$ is the absorption of an isolated atom. Rearranging, the fine structure is given by

$$
\Upsilon(E)=\frac{\mu(E)-\mu_{0}(E)}{\mu_{0}(E)}
$$


The XAFS, $x(k)$, for K-edge absorption may be expressed as a function of photoelectron wavevector, $k=\sqrt{2 m\left(E-E_{0}\right)} / \hbar$, as

$$
\backslash(k)=\sum_{i} B_{i}(k) \sin \left[2 k R_{i}+2 \delta(k)+\phi_{i}(k)\right] .
$$

where the sum is taken over all atoms. $i$, located at distances $R_{i}$ from the absorbing atom. Phase shifts, $\delta(k)$ and $\phi_{i}(k)$, arise from the interaction with the absorber and the backscatterer, respectively.

The amplitude function. $B_{i}(k)$. is

$$
B_{i}(k)=\frac{N_{i}^{*}}{k R_{i}^{2}} S_{0}^{\prime 2} F_{i}(k) e^{-2 R_{i} / \lambda_{v}(k)} \epsilon^{-2 k^{2} \sigma_{i}^{2}} .
$$

where

$$
N_{i}^{*}=3 N_{i} \cos ^{2}\left(\theta_{i}\right)
$$

Here $V_{i}$ is the number of atoms of type $i$ located at an average distance $R_{i}$ from the absorber. The factor $\cos ^{2}\left(\theta_{i}\right)$ is related to the polarization dependence of the li-edge XAFS, where $\theta_{i}$ is the angle between the electric vector and the bond between the absorber and the backscatterer (recall that the absorption depends on the dipole operator. $\overrightarrow{\mathcal{E}} \cdot \vec{r}) . S_{0}^{2}$ is the passive electron factor describing the overlap between the wavefunctions of the electrons in the absorbing atom before and after the ejection of the core photoelectron $\left(S_{0}^{2} \leq 1\right)$. The backscattering amplitude of atom $i$ is given by $F_{i}(k), e^{-2 k^{2} \sigma_{1}^{2}}$ is the XAFS Debye-Waller factor where $\sigma_{i}^{2}$ is the mean-square relative displacement about the average distance $R_{i}$, including thermal and static disorder. and $\lambda_{i}(k)$ is the mean free path of the photoelectron [40].

Historically, XAFS data were analyzed by comparing the spectrum for the sample of interest to a reference compound (or several compounds) containing the same absorbing and backscattering species, approximately the same distance apart as in the unknown sample. In this method of analysis the XAFS appears as

$$
\backslash(k)=\frac{N_{i}}{k R_{i}^{2}}\left|f_{i}(k)\right| \sin \left[2 k R_{i}+\phi_{i}(k)\right] \text {. }
$$

where the empirical phase, $\phi_{i}(k)$. and amplitude, $\left|f_{i}(k)\right|$ are determined from the standard sample. The data are then curve-fitted to determine the nearest neighbour distances, $N_{i}$, and the bond lengths, $R_{i}$. 
The radial distribution function may be obtained by taking the Fourier transform of $y(k)[42]$. The realization of this point constituted a major step in the development of modern XAFS. The functional form of the Fourier transform is

$$
F T\left[k^{p} \backslash(k)\right]=\frac{1}{\sqrt{2 \pi}} \int_{k_{\min }}^{k_{\max }} d k w^{\prime}\left(k^{\prime}\right) k^{p} \backslash(k) e^{\left(2 i k R_{s}\right)} .
$$

where $k_{\min }$ and $k_{\text {max }}$ are the limits of the data and $p$ is the power of the $k$-weighting used to emphasize a particular range of the spectrum. Due to the finite range of the data in $k$-space. a transform window. $w(k)$. is used to minimize transform artifacts. In this thesis a $10 \%$ Gaussian window was used. having the form

$$
w(k)=\epsilon .5 p\left[\left(\frac{2\left(k-k_{m i d}\right)}{\Delta k}\right)^{2} \ln (0.1)\right] .
$$

where the data are taken over the interval $\Delta k=k_{\operatorname{mar}}-k_{\text {min }}$. and $k_{\text {mid }}$ is the midpoint of the range.

Recent developments have enabled the calculation of theoretical XAFS spectra for any arrangement of atoms, even those for which no reference compound exists [-1:3]. An example of such a calculation package is FEFF6b which is used in this thesis [44]. Details of this method of data analysis appear in ('hapter $\pi . T$.

Having now reviewed the principles of XAFS. in the next section we address the details of the glancing incidence geometry used for polarization-dependent studies.

\subsection{The Glancing Incidence Geometry}

In the glancing (or grazing) incidence geometry, polarized photons from a synchrotron source are incident on a specimen at very shallow angles. The photon energy is then varied in the usual way to measure the XAFS. Surface or near-surface measurements are now routinely performed in this geometry with incident angles, $\theta$, in the neighbourhood of the critical angle for total external reflection, $\theta_{C}$. The theoretical basis for evaluating the electric field amplitudes within samples aligned in this geometry has been summarized by Jiang [45]. In this section an overview of the physics will be given in order to calculate the field intensities in layered semiconductor crystals. 


\subsubsection{Parratt's Formulation}

A method for calculating the glancing incidence reflectivity in stratified, layered systems was developed by Parratt [46] some time ago. More recent theories have been published by Kohn [4i] and others. In the present discussion the former approach will be used. Although Kohn's transfer matrix method is more elegant. the physical basis is the same.

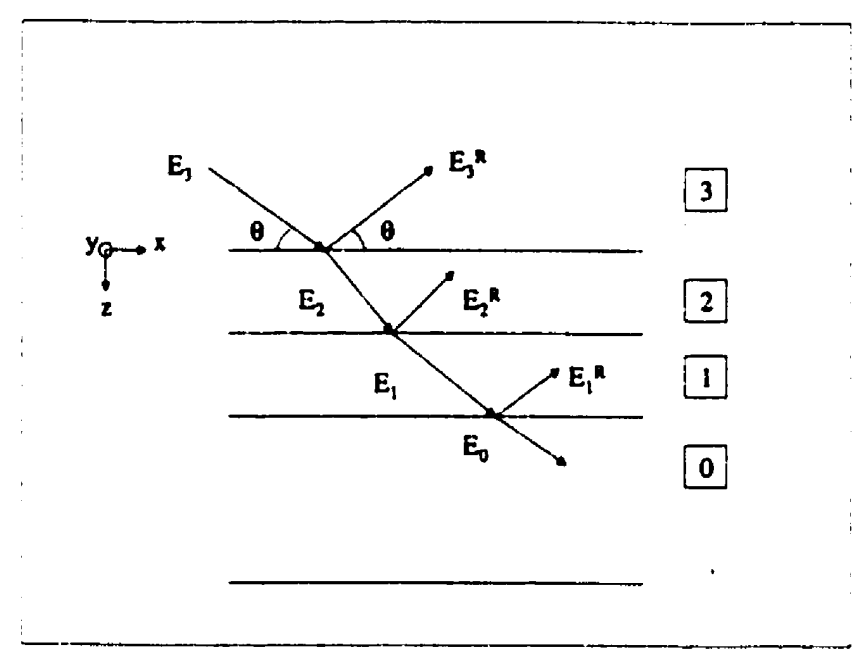

Figure 4.3: The propagation of a plane wave in a layered structure. In this example layer 3 corresponds to the vacuum above the system, while the substrate is layer 0. Note that the electric field amplitudes refer to the values at the centre of each layer. while the $z$ coordinate is also measured from the centre of the layer.

Consider a layered structure as shown in Figure 4.3. The substrate is taken as layer $m=0$, with increasing layer indices along the growth (-ve $z$ ) direction. For the system depicted here the vacuum space above the sample is denoted by $m=3$. with corresponding amplitudes $E_{3}$ and $E_{3}^{R}$ for the incident and reflected electric field components, respectively. In the following discussion the $\sigma$-polarization will be assumed, although the same results are obtained for the $\pi$-polarization ( $P=1$ for $\sigma$, and $P=\cos 2 \theta_{B} \sim 1$ for $\pi$ polarization with $\theta_{B} \sim 0$ ). The electric vector thus points out out of the page in the $y$-direction. 
In vacuum the wavevector, $\vec{k}$ is related to the frequency, $\omega$, by $k=|k|=\omega / c$. In a medium with complex index of refraction, $n_{i}$, the wavevector is $k_{i}=n_{i} \omega / c$. For the glancing incidence geometry the index of refraction is related to the $(000)$ structure factor, $F_{0}$. Including the dispersive. $\delta$, and absorptive, 3 , parts of the index of refraction, we have

$$
n=1-\delta-i 3
$$

where

$$
\delta=\frac{1}{2} \Gamma F_{0}^{\prime}=\frac{1}{2} \backslash_{0}^{\prime}=\frac{N_{A}}{2 \pi} \frac{\epsilon^{2}}{m c^{2}} \frac{\rho}{.1} \lambda^{2}\left(f_{0}+f^{\prime}\right) .
$$

and

$$
3=\frac{1}{2} \Gamma F_{0}^{\prime \prime}=\frac{1}{2} \lambda_{0}^{\prime \prime}=\frac{N_{A}}{2 \pi} \frac{\epsilon^{2}}{m c^{2}} \frac{\rho}{.1} \lambda^{2}\left(f^{\prime \prime}\right) .
$$

where $F_{0}^{\prime}$ and $F_{0}^{\prime \prime}$ are the real and imaginary parts of the $(000)$ structure factor with $\Gamma$ as defined in Section 3.1. In the above. $N_{A}$ is Avogadro's number, $\rho$ is the mass density of the material with atomic weight $M$ and $\lambda$ is the X-Ray wavelength. In this discussion the layers are considered homogeneous. so that the only change in material occurs along the $z$-direction. perpendicular to the surface. The critical angle is determined from the relation $\theta_{C}=\sqrt{2 \delta}$.

For a monochromatic, plane $X$-Ray wave incident on the system with wavevector $\vec{k}$ in the $x, z$-plane the wave is reflected and refracted at the boundaries. Thus. both refracted and reflected plane waves propagate in each layer. It is assumed that these are the only two such waves in the material. For the substrate (assumed infinitely thick) no reflected beam propagates. Within each layer the $x$-components of the two waves are the same, while the $z$-components have opposite signs. The tangential components, $k_{x_{1}}$, of the wavevectors are equal on either side of a boundary, while the $z$-components are different in the different media due to the different electric susceptibilties.

The electric field vector in layer $m$ is written [45]

$$
\vec{E}_{m}(x, z, t)=E(x, t)\left\{\vec{E}_{m} \exp \left(-i k_{z m} z\right)+\vec{E}_{m}^{R} \exp \left(i k_{z m} z\right)\right] .
$$

where $E(x, t)=\exp \left[i\left(\omega t-k_{r} x\right)\right]$. Here $\vec{E}_{m}$ and $\vec{E}_{m}^{R}$ are the amplitudes of the refracted and reflected waves in the middle of the layer and the coordinate $z$ is measured from the middle of the layer. 
For a small angle of incidence. $\theta$. at the top interface the wavevector may be approximated by $k_{x_{3}} \approx k_{3}$, and so

$$
k_{z_{2}} \approx k_{3}\left(\theta^{2}-2 \delta_{2}-2 i 3_{2}\right)^{1 / 2} .
$$

Using the notation

$$
f_{m}=\left(\theta^{2}-2 \delta_{m}-2 i 3_{m}\right)^{1 / 2},
$$

the general relationship between the wave number of the $m$ th layer and that of vacuum is

$$
k_{s_{m}}=k_{3} f_{m} .
$$

In the $m$ th layer, the refracted beam is now

$$
E_{m}\left(z_{m}\right)=E_{m}(0) \exp \left[i\left(\omega t-k_{x_{m}} x_{m}\right)\right] \exp \left(-i k_{3} f_{m} z_{m}\right) .
$$

The conditions of continuity for the electric and magnetic fields at the boundary between layers $m$ and $m-1$ can be written

$$
a_{m} E_{m}+a_{m}^{-1} E_{m}^{R}=a_{m-1}^{-1} E_{m-1}+a_{m-1} E_{m-1}^{R} .
$$

and

$$
\left(a_{m} E_{m}-a_{m}^{-1} E_{m}^{R}\right) f_{m}=\left(a_{m-1}^{-1} E_{m-1}-a_{m-1} E_{m-1}^{R}\right) f_{m-1} .
$$

Here $a_{m} \equiv \exp \left(-i k_{s_{m}} d_{m} / 2\right)$. where $d_{m}$ is the thickness of the $m$ th layer [45].

At the $m, m-1$ interface the reflection, $R_{m}$, and transmission coefficients, $T_{m}$, are defined as

$$
R_{m}=\frac{a_{m}^{-1} E_{m}^{R}}{a_{m} E_{m}}
$$

and

$$
T_{m}=\frac{a_{m-1}^{-1} E_{m-1}}{a_{m} E_{m}} .
$$

Note that the amplitudes $a_{m} E_{n}, a_{m}^{-1} E_{m}^{R}$ and $a_{m-1}^{-1} E_{m-1}$ are the values of the incident. reflected and refracted beams, respectively, measured at the interface.

This leads to the recursion relations

$$
R_{m}=\frac{a_{m-1}^{4} R_{m-1}+G_{m}}{a_{m-1}^{4} R_{m-1} G_{m}+1}
$$


and

$$
T_{m}=\frac{1+R_{m}}{1+a_{m-1}^{4} R_{m-1}}
$$

where

$$
G_{m}=\frac{f_{m}-f_{m-1}}{f_{m}+f_{m-1}} .
$$

'l'hus if $K$ at one interface is known, then $K$ and $T$ ' at every interface can be calculated. At the bottom of the substrate there is neither a reflected beam nor a transmitted beam. i.e. $R_{0}=0$ and $T_{0}=0$. In this way the recursion relations can be applied to successive layers from bottom to top to determine $R$ and $T$ at every interface. The reflectivity at the top surface is then given by (using the notation of Figure 4.3)

$$
\left|R_{3}\right|^{2}=\left|\frac{E_{3}^{R}}{E_{3}}\right|^{2} \text {. }
$$

where $\left|a_{3}\right|=1$ and $f_{3}=0$, where the index $n_{3}=1$.

It is useful to derive a method for calculating the amplitude of the electric field at a given position within the layered structure. From the definitions of the reflection and transmission coefficients at the $m . m-1$ boundary, we have

$$
E_{m-1}=T_{m} a_{m} a_{m-1} E_{m}
$$

and

$$
E_{m}^{R}=R_{m} a_{m}^{2} E_{m}
$$

Referring to Figure 4.3 we may now evaluate the field amplitudes relative to the amplitude, $E_{\text {incoming }}$ of the incident wave.

\subsubsection{Field Amplitudes in the Crystal}

As mentioned earlier the transfer matrix method of hohn [4i] is a straightforward and tidy method of dealing with systems composed of many layers. For SQW structures it is possible to calculate the wave amplitudes (e.g. at the location of the In atoms) based on the principles outlined in the preceeding section. For more complicated structures, however, the matrix method is preferred. 
In the topmost medium, the $n$th layer, the "refracted" field amplitude is

$$
E_{n}=E_{\text {incoming }} .
$$

From equation 4.28. the amplitude of the reflected wave is found from the boundary condition $(\mathrm{BC})$ at the $n . n-1$ interface

$$
E_{n}^{R}=R_{n} a_{n}^{2} E_{n}
$$

In medium $n-1$, the top interface $\mathrm{BC}$ gives

$$
E_{n-1}=T_{n} a_{n} a_{n-1} E_{n} \text {. }
$$

while the bottom interface $\mathrm{BC}$ gives

$$
E_{n-1}^{R}=R_{n-1} a_{n-1}^{2} E_{n-1}=\left(R_{n-1} a_{n-1}^{2}\right)\left(T_{n} a_{n} a_{n-1} E_{n}\right) .
$$

For the $n-2$ layer we similarly find

$$
E_{n-2}=T_{n-1} a_{n-1} a_{n-2} E_{n-1}
$$

and

$$
E_{n-2}^{R}=R_{n-2} a_{n-2}^{2} E_{n-2}=\left(R_{n-2} a_{n-2}^{2}\right)\left(T_{n-1} a_{n-1} a_{n-2}\right) E_{n-1} .
$$

Now, using equation 4.31

$$
E_{n-2}^{R}=\left(R_{n-2} a_{n-2}^{2}\right)\left(T_{n-1} a_{n-1} a_{n-2}\right)\left(T_{n} a_{n} a_{n-1}\right) E_{n} .
$$

In the $\mathrm{GaAs}(001) / \mathrm{InAs} / \mathrm{GaAs}$ structures studied in this work, the $(n-2)$ th layer is the quantum well $(m=1)$. The ratio of the total field amplitude in this layer. relative to the incoming field amplitude is then

$$
\frac{E_{1}^{\text {tot }}}{E_{\text {incoming }}}=\left(1+R_{1} a_{1}^{2}\right)\left(a_{1}\right)\left(T_{2} a_{2}^{2}\right)\left(T_{3} a_{3}\right),
$$

which is easily calculated from the reflection and transmission coefficients.

Figure 4.4 shows the results of calculations carried out for layered structures of this type with InAs layers $1 \mathrm{ML}$ thick and GaAs caps of varying thicknesses. The 
calculations were performed for an incident photon energy of $200 \mathrm{eV}$ above the In $\mathrm{K}$ edge energy $\left(E_{0}=27940 \mathrm{eV}\right)$. The structure factors were calculated using the method of Appendix A. It is interesting to note the tremendous increase in the field amplitude at the surface observed at the substrate critical angle. This results from the appearance of an X-Ray standing wave in the crystal as will be discussed. On the linear scale it is not possible to distinguish between the reflectivities for samples with different cap thicknesses. On the logarithmic scale of Figure 4.5 the differences become more apparent.

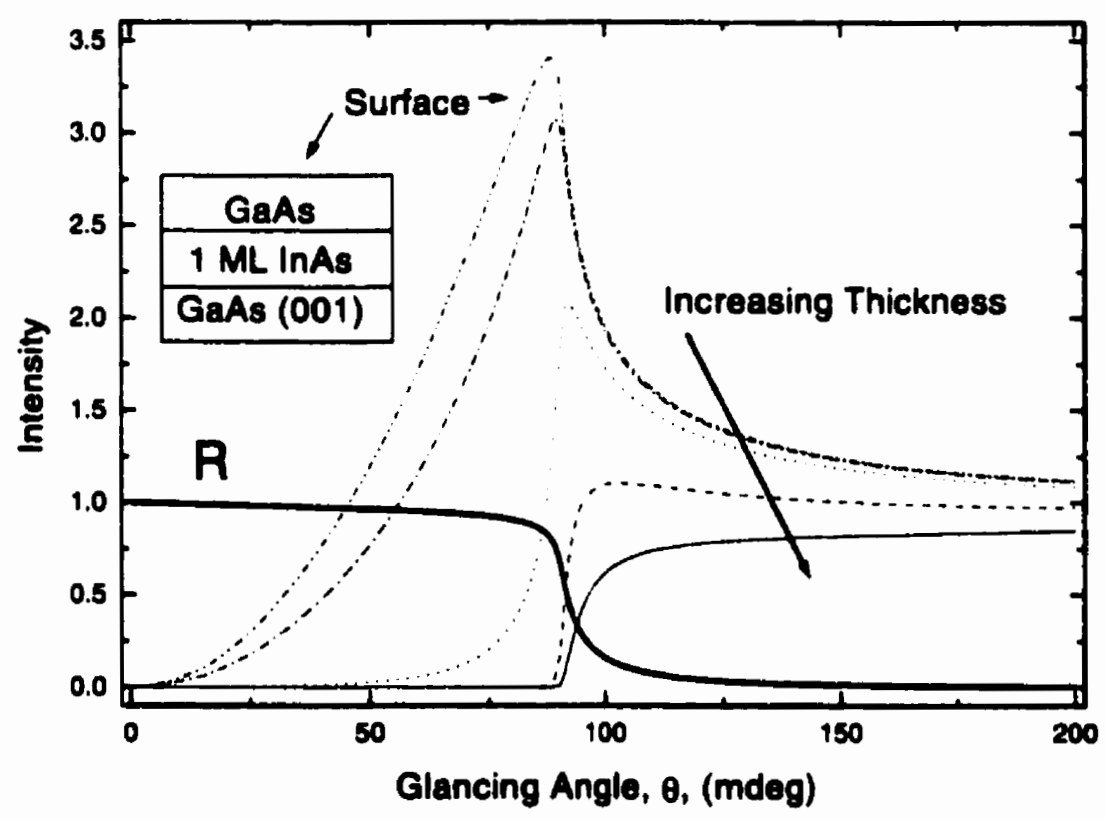

Figure 4.4: The reflectivity, $R$, calculated for layered InAs/GaAs structures as described in the text. The electric field intensity at the location of the In atoms is shown for $\mathrm{GaAs}$ cap thicknesses of $10 \AA, 100 \AA, 500 \AA$ and $1000 \AA$. The largest intensity depicted in the figure corresponds to the value obtained at the topmost surface of the structure.

It is also important to consider the penetration depth of the X-Rays into the 


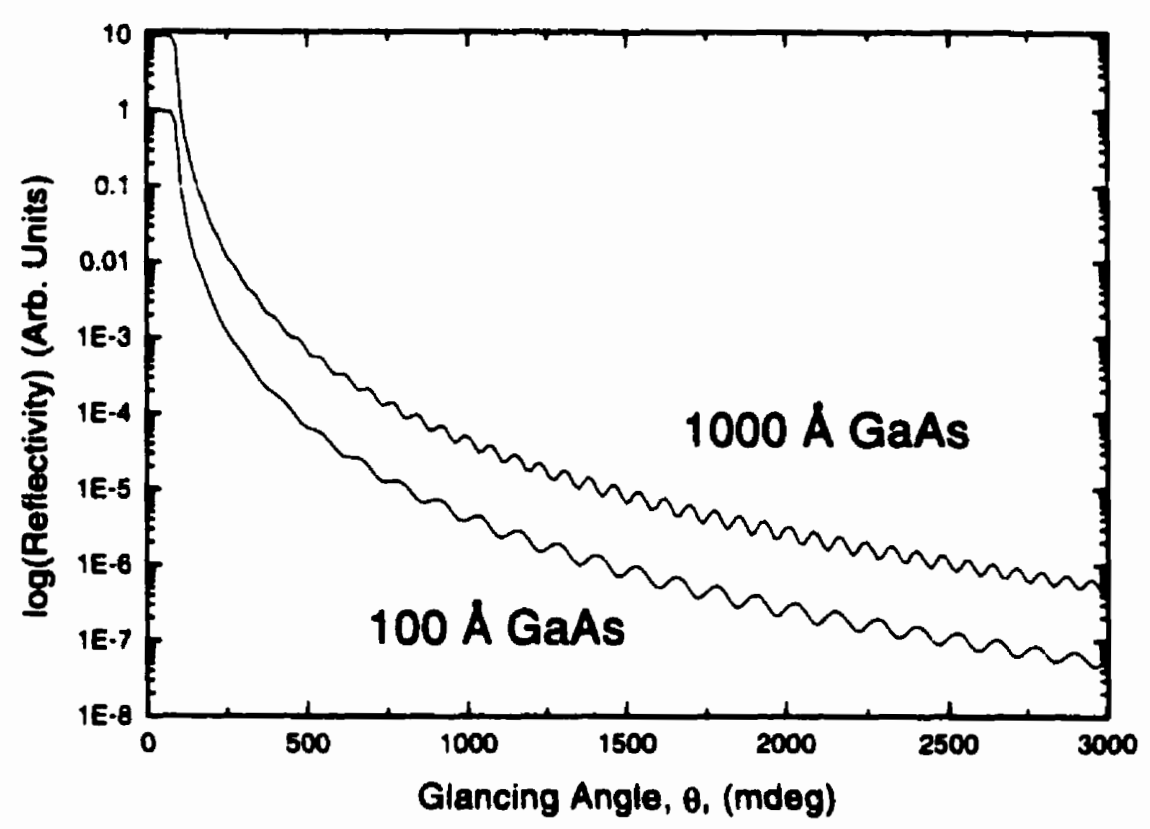

Figure 4.5: The reflectivity, $R$. calculated for layered InAs/GaAs structures with GaAs cap thicknesses as indicated. The curves were offset by one decade for clarity. Note the very wide angular range of the figure.

crystal as a function of incidence angle. If we define [48] the damping constant. $z_{0}$ as

$$
z_{0}=\frac{\sqrt{2}}{k}\left[\sqrt{\left(\theta^{2}-2 \delta\right)^{2}+43^{2}}-\left(\theta^{2}-2 \delta\right)\right]^{-1 / 2} .
$$

then it is seen from Figure 4.6 that below the critical angle the energy does not penetrate very far into the crystal. This allows for $\mathrm{X}$-Ray absorption studies of atoms located very near the surface in the so-called "total reflection" geometry. For InAs films buried beneath a GaAs capping layer, however, a slightly deeper incidence angle was required, to ensure that a significant amount of energy propagates through to the location of the In atoms. This will be discussed in more detail in Section 6. 7 .

It is worth pointing out that for a fixed angle of incidence the reflectivity does not necessarily remain constant during an XAFS scan. As the incident photon energy 
is increased through an absorption edge the changing absorption may significantly alter the reflectivity which can then exhibit EXAFS. In the present case, however, the reflectivity is due largely to the GaAs substrate, and the changing photoabsorption from the dilute In atoms has a negligible effect on the reflectivity. A discussion of "reflectivity EXAFS" has been given by Jiang [45].

\subsubsection{Total Reflection XSW}

In the glancing incidence geometry it is possible to create an XSW field with varying periodicity above a surface [49]. Although this method was not employed in the present study. it is nevertheless worth discussing. As in the Bragg geometry the interference of the two coherent traveling plane waves creates a standing wave with planes of maximum intensity parallel to the surface and with a period

$$
D=\frac{\lambda}{2 \sin \theta} \text {. }
$$

Figure 4.7 shows the reflectivity and phase of a Ciads crystal as functions of the incidence angle. The phase. $\alpha$. decreases from $\pi$ to 0 as $\theta$ is increased from 0 to $\theta_{C}$ '. From Equation 4.38. the first antinode occurs at infinity for $\theta=0$. As $\theta$ is increased through the range of total reflection, the first antinode moves inwards until it coincides with the crystal surface at $\theta=\theta_{C}$. As the period decreases throughout this angular range, the remaining antinodes move inwards like a compressing bellows [49]. The compression of the standing wave and the inwards shift, due to the phase change are expressed in terms of the $E$-fieid intensity above the surface as

$$
I(\theta . z)=\left|E_{0}\right|^{2}[1+R+2 \sqrt{R} \cos (\alpha-2 \pi Q z)] .
$$

Here $Q=2 \sin \theta / \lambda=1 / D$ is the magnitude of the wavevector transfer. Figure $4 . i$ also show the $\theta$-dependence of the intensity, $I$, for $z=0$ and $z=2 D_{C}$, where the critical period. $D_{C}=\lambda / 2 \theta_{C}$, is the standing wave period at the critical angle. Standing wave experiments performed in this manner [49] have been used to locate metal atoms up to $200 \AA$ above mirror surfaces with a precision of $2 \AA$. 


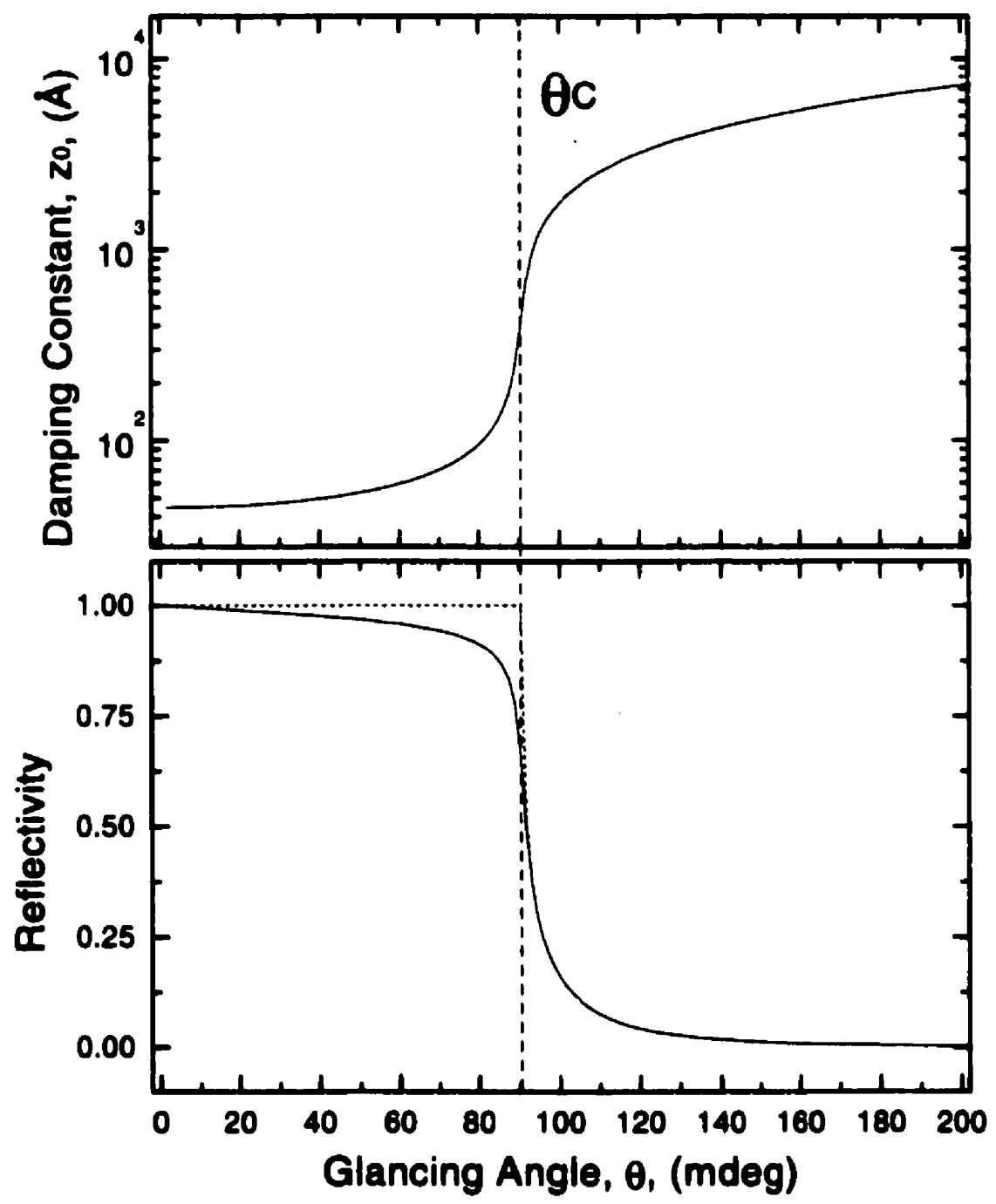

Figure 4.6: The reflectivity, $R$. calculated at $28140 \mathrm{eV}$ for a GaAs crystal and the penetration depth of the X-Rays into the crystal. The dotted line indicates the reflectivity which would be obtained for a non-absorbing crystal where $R=1$. 


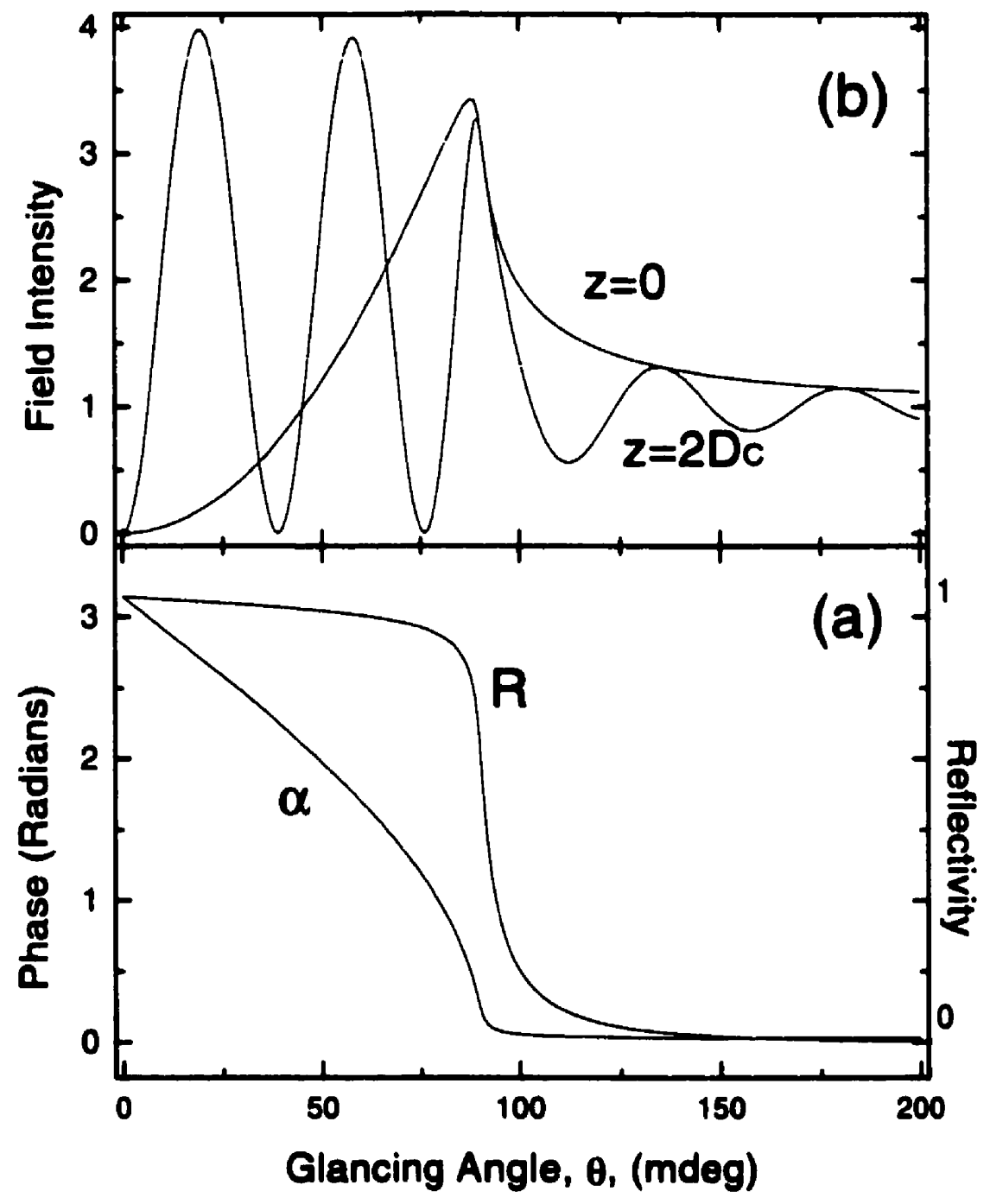

Figure 4.7: (a) The reflectivity, $R$, and phase, $\alpha$, for a GaAs crystal. (b) The electric field intensity at the crystal surface $(z=0)$ and at twice the critical period $\left(z=2 D_{C}\right)$. 
The next chapter will summarize previously reported work dealing with In.As layers in GaAs. This will include fundamental issues of strain. epitaxy and optical characterization. 


\section{Chapter 5}

\section{Review of Literature}

In this chapter published reports dealing with the growth and characterization of In.As layers in GaAs will be discussed. As mentioned. the different lattice parameters of InAs and CiaAs produce a biaxial strain in the epitaxial structure. In the first section this topic will be discussed, first though a summary of the macroscopic elastic description of the strain accommodation. followed by a review of experiments aimed at direct measurement of the physical properties of such strained lavers. In practice the fabrication of very abrupt InAs layers is hindered by In segregation during the CiaAs cap layer growth. Previously this process was studied almost exclusively in samples grown by MBE, due largely to the more extensive range of techniques available for in-situ studies in UHV. The present work is thus somewhat unique in addressing this phenomenon in MOVPE samples. In the MOVPE environment the predominant insilu characterization technique is RDS which will be discussed in Section 5.3. Finally. optical studies of ultrathin InAs layers in GaAs will be discussed. This will include both theoretical and experimental works aimed at describing the emission properties of these structures.

\subsection{Strain in Heteroepitaxy}

In the design of semiconductor devices, it is often desirable to use lattice-mismatched layered heterostructures. The SQW structures studied in this work are a particularly 
striking example of this, given the bulk lattice constants of GaAs $\left(a_{G a A s}=5.6532 \mathrm{~A}\right)$ and InAs $\left(a_{\ln A s}=6.0584 \AA\right)$ which differ by $\sim 7 \%$. Long ago, the theoretical bases for such heteroepitaxial growth were studied. There are three principal growth modes: layer-by-layer (Frank-van der Merwe); layer-by-layer plus island nucleation (StranskiKrastanov); and 3-dimensional nucleation (Volmer-Weber) [50]. InAs grows on GaAs (001) in the layer-by-layer mode for approximately 1.5 MLs, after which a transition to the Stranski-Krastanov mode occurs to relieve the strain. InAs "quantum dots" grown by this method are candidates for 0 -dimensional quantum confinement and this is an extremely active field of research at this time. The present discussion considers those aspects of strain which are relevant to the heterostructures of interest to this thesis.

\subsubsection{Macroscopic Elastic Theory}

The theory of materials elasticity is well established [51]. For an epitaxial layer grown coherently on a substrate, i.e. without misfit dislocations, the state of elastic strain may be described by the macroscopic elastic theory (MET) developed by Hornstra and Bartels [52]. They considered strain in III- $V$ compounds for growth planes perpendicular to a symmetric axis (e.g. (001)) as well as for arbitrary growth planes. such as (113).

For an epitaxial layer much thinner than the substrate it may be assumed that there is negligible strain in the substrate. Consider an epilayer in its bulk state. and suppose it is strained in all directions by an amount

$$
\epsilon_{\|}=\left(\frac{\Delta a}{a_{f}}\right) \text {, }
$$

where $\Delta a=a_{s}-a_{f}$ and $f$ denotes the film layer, $s$ denotes the substrate. If the layer is coherently fitted to the substrate $\left(a_{\|}=a_{s}\right)$ and the stresses applied to the surface are removed, then the strain parallel to the surface is still $\epsilon_{\|}=\left(a_{\|}-a_{f}\right) / a_{f}$, but the perpendicular strain has a different value, $\epsilon_{\perp}=\left(a_{\perp}-a_{f}\right) / a_{f}$. An additional shear strain will be present if the surface normal is not aligned along a symmetry axis. The components of strain in the two directions are related through the elastic constants 
of the epilayer, $C_{11}$ and $C_{12}$, as

$$
\epsilon_{\perp}=-2\left(\frac{C_{12}}{C_{11}}\right) \epsilon_{\| 1}
$$

Notice that $\epsilon_{\perp}$ has the opposite sign to $\epsilon_{\|}$. The epitaxial layer now has a perpendicular lattice constant different from the substrate. while the in-plane lattice constants are equivalent. An illustration of the strain accommodation is given in Figure 5.1.

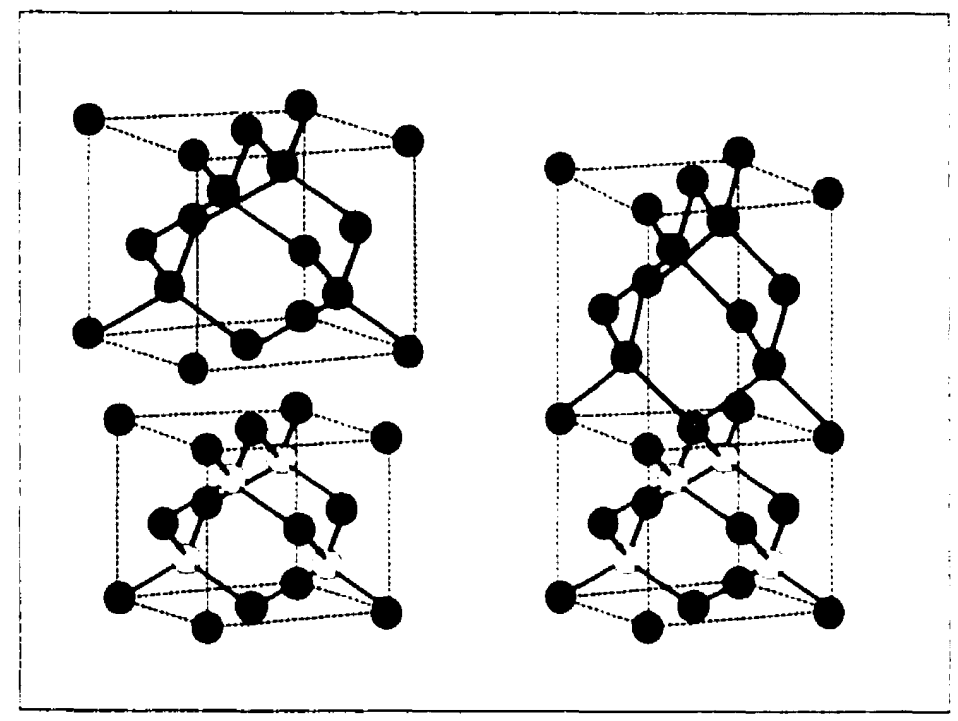

Figure 5.1: On the left-hand side the bottom and top images denote a substrate (e.g. GaAs) and an epilayer (e.g. InAs) with a larger lattice constant. The right-hand side illustrates the strain accommodation through a tetragonal distortion of the InAs unit cell. In, $\mathrm{Ga}$ and $\mathrm{As}$ atoms are indicated by black, white and shaded circles. respectively.

Assuming the validity of the MET we can evaluate the effects of strain on a single ML InAs film buried in $\operatorname{GaAs}(001)$ [2]. The elastic constants of InAs and GaAs are given in Table 5.1. From Equations 5.1 and 5.2 the perpendicular lattice constant of the In As layer is $a_{\perp}=6.4988 \AA$. The corresponding In- $A$ s plane spacing in the (001)-direction is $1.624 i \AA$ which is stretched by $\sim 15 \%$ compared to the value for GaAs. In the bulk InAs lattice with dimension $a_{\text {InAs }}$, the nearest-neighbour bond 


\begin{tabular}{|c|c|c|}
\hline \hline Material & $C_{11}\left(10^{10} \mathrm{Nm}^{-2}\right)$ & $C_{12}\left(10^{10} \mathrm{Nm}^{-2}\right)$ \\
\hline GaAs & 11.9 & 5.38 \\
\hline InAs & 8.329 & 4.526 \\
\hline \hline
\end{tabular}

Table 5.1: Elastic constants of bulk compound semiconductors. From Reference [53].

length is $K_{I n-A s}=\sqrt{3} a_{I n A s} / 4=2.62: 3 \mathrm{~A}$. For the single layer inserted into a CiaAs matrix a simple geometric calculation predicts the strained bond length to be slightly compressed, having the value $R_{I n-A s}^{\prime}=2.576 \mathrm{~A}$.

For ternary (e.g. $\operatorname{In}_{r} \mathrm{Ga}_{1-r} A s$ ) or quaternary materials (e.g. $\ln _{x} \mathrm{Ca}_{1_{-r}} A \mathrm{~s}_{1-y} \mathrm{P}_{y}$ ) the bulk lattice constants are given by Vegard's law. For an $\ln _{r}\left(\mathrm{a}_{1-r} \mathrm{As}\right.$ film.

$$
a_{f}=x a_{l n d s}+(1-x) a_{C i a A s} .
$$

in terms of the bulk lattice constants of the constituents. Similarly. the elastic constants of the film are given by the concentration-weighted elastic constants of InAs and GaAs.

\subsubsection{Bond-Lengths in Semiconductor Alloys}

In the interpretation of powder diffraction data from ternary alloys the so-called "virtual crystal approximation" (VCA) may be used. For an $\mathrm{In}_{r} \mathrm{Ga}_{1-r}$ As film, this supposes that the In and Ga atoms occupy the same (virtual crystal) positions and that Vegard's law applies. A pioneering study by Mikkelsen and Boyce [54] used EXAFS to study the $\mathrm{In}-\mathrm{As}$ and $\mathrm{Ga}-\mathrm{As}$ bond lengths in a series of powder samples with varying compositions, $x$. The data showed unequivocally that although the $\mathrm{VCA}$ is adequate for diffraction studies. it does not describe the microscopic bond lengths in the alloyed material.

Using K-edge EXAFS for all three elements in the samples, Mikkelsen and Boyce found that the In-As and Ga-As nearest-neighbour bond lengths in an alloy are closer to their respective distances in the pure compounds than to the predictions of the VCA. Their results may be summarized as follows. (i) only small changes in the first neighbour distances were observed; (ii) the widths of nearest-neighbour peaks 
in the Fourier Transform of $\chi(k)$ are not broadened by the random solid disorder: and (iii) cation-cation distances peak at a single distance in good agreement with the $\mathrm{VCA}$, while the anion-anion distribution is bimodal, indicating that the anions do not occupy VCA positions. Moreover, the second nearest-neighbours were altered in the alloy as a result of disorder on the cation sublattice. Thus $\mathrm{In}_{x} \mathrm{Ga}_{1-r} \mathrm{As}$ alloys are not virtual crystals at the atomic level, while the cation sublattice does approach a virtual crystal beyond the second-neighbour distance [54].

\subsubsection{Structural Studies of InAs SQWs}

Many HRXRD studies of thin, buried layers have been reported in the literature. Different approaches have been used to model the data. including the semikinenatical approach of Tapfer and Ploog [55, 56]. and full dynamical treatments [57]. The main advantage of the semikinematical approach compared with a full dynamical treatment is a decrease in the computation time, at the expense of some precision. Modern personal computers are now sufficiently fast that these approximations are unnecessary. Furthermore. Shufan and Zhenhong have shown that the semikinematical approach is inadequate for structures of any complexity when compared with the full dynamical theory [58].

Using Tapfer's technique, Brandt et al. demonstrated the sensitivity of HRXRD to 1 ML InAs films in GaAs with very high resolution [10]. The "flashoff" technique used for preparing the samples will be discussed in some detail in Section 5.2. XRD measurements were obtained using the (004) and (224) Ga.As substrate reflections. Recall that a full dynamical diffraction simulation for this type of structure was shown in Figure 3.7.

In these measurements the incident X-Ray wave is scattered by the GaAs substrate and the cap simultaneously at the Bragg angle. The sandwiched InAs layer causes a phase shift between these two waves which produces the observed Pendellösung fringes. The period of these fringes is directly related to the cap layer thickness. This interference effect is influenced by the lattice strain and thickness of the InAs layer, although the layer thickness and composition are highly correlated parameters 
making it difficult to distinguish between a single, well-defined InAs ML and two MLs of $\operatorname{In}_{0.50} \mathrm{Ga}_{0.50}$ As, for example. Moreover, the sensitivity of the technique to the InAs layer thickness is reduced as the GaAs cap thickness is decreased. Reference [10] reported an InAs layer thickness of $3.9 \pm 0.15 \mathrm{~A}$, corresponding to $1.2 \pm 0.05 \mathrm{ML}$. This calculation used the MET as described in Section 5.1.1, assuming that the lattice mismatch was accommodated entirely by an elastic tetragonal distortion of the InAs unit cell.

Some confusion arose in the literature due to a subsequent paper by Brandt $t$ al. in which high-resolution electron microscopy (HREM) data was supposed to reveal a breakdown of the MET in the limit of ML films [18]. It was reported that although the MET agreed with the results for films 3 ML thick, the HREM images were consistent with a strain of $13 \%$ for a single ML. This is nearly twice the value predicted by the MET and suggests In-As bond length conservation for a single InAs ML.

In-As bond-length conservation has been suggested by some theoretical work. An ab initio total energy calculation predicted that the $\mathrm{ln}-\mathrm{A}$ s bond length for a buried ML in GaAs was only slightly shorter than in the bulk material [59]. Later a valenceforce field (VFF) model predicted In-As bond-length conservation similar to Brandt's experimental result $[60]$

More recently, local density approximation (LDA) [61] and random cluster [62] calculations have predicted strained bond-lengths which agree with the MET. as well as with other experimental results.

Giannini et al. reported the first XSW study of a single InAs layer buried in GaAs(001) [1]. Using the "flashoff" technique (Section 5.2) they deposited 1.08 ML of InAs. capped by nominally $275 \AA$ of GaAs, and determined the layer thicknesses with HRXRD using the GaAs (224)-reflection. Their synchrotron XSW measurements at fixed photon energy, using the reflectivity and the $I n-L_{\alpha}$ fluorescence, yielded the results $P^{H}=1.17 \pm 0.02, F^{H}=0.58 \pm 0.0 \tau$. While the Debye-Waller factor is expected to reduce the measured $F^{H}$ somewhat, the measured value suggested a high degree of static structural disorder. An alternative interpretation was adopted based on the method described in Section 3.2.2. Given the tendency for In segregation during the cap layer growth (Section 5.2) the measured values of $P^{H}$ and $F^{H}$ were explained by 
the incorporation of In into "terraces". Using the MET, a single. highly-strained InAs layer in GaAs would have $P^{H}=1.15$. Giannini et al. supposed that the effect of In segregation was to distribute the In atoms into three terraces, with $75 \%$ of the atoms located at $P_{1}^{H}=1.15,20 \%$ of the atoms at $P_{2}^{H}=3.45$ and the remaining $5 \%$ of the atoms at $P_{3}^{H}=5.75$, relative to the last As-plane of the substrate. Equations 3.543.57 verified that the experimental results were consistent with this interpretation. The authors were careful to emphasize that the "terrace nucleation" was different from a graded $\operatorname{In}_{x} \mathrm{Gia}_{1-x}$ As alloy [1].

HRXRD studies of single and even subML InAs films buried in GaAs were also reported by Alonso et al. [13]. They used symmetrical and asymmetrical reflections to study In As layers of thickness 0.4-3.2 A, capped by $1800 \mathrm{~A}$ of GaAs deposited on Ga.As (001)- and (311)-oriented substrates, deposited by MBE.

Grazing incidence diffraction (GID) using synchrotron X-Rays was emploved by Rose et al. to study InAs layers of thickness 0.7-4 ML. buried in GaAs [63]. The films were deposited by MOVPE at $650^{\circ} \mathrm{C}$ using TMIn. TMGa and $\mathrm{AsH}_{3}$. In the GID measurements the diffracted beam was recorded at an angle nearly perpendicular to the plane of incidence. while the incident beam reflected from each sample at a glancing angle. For exit angles greater than the critical angle, sharp interference fringes were observed in the diffracted intensity. While these interference fringes should. in principle, be related to the layer thicknesses, the Cia.As caps were found to have thicknesses only $\sim 84 \%$ of their nominal values, while the InAs layers were found to have less than half of their nominal thicknesses. These results could have been verified by comparison with more conventional HRXRD measurements.

Bocchi and Ferrari used HRXRD with several substrate reflections to study the distribution of In atoms in InAs SQWs in GaAs(001) grown by MBE and ALMBE [64]. While the symmetrical (004)-reflection is rather insensitive to the distribution of In atoms, it was shown that the asymmetrical (224)-reflection in the grazing exit geometry could be used to evaluate the distribution of In atoms along the growth direction. It was reported that the measured XRD profiles were consistent with In segregation to a depth of $\sim 6 \AA$, using Tapfer's approach [55]. In the article the authors show a 
schematic of the segregated In concentration as a function of depth within the crystal. Strangely, this illustration seems to suggest a series of In-containing layers with fractional ML thicknesses. While the physical interpretation of this is unclear, it is also unclear from our dynamical simulations that the experiment could distinguish between single InAs layers and the segregated profiles, as claimed in the article.

To clarify the microscopic structure of InAs layers buried in GaAs. Woicik $t$ t al. combined XSW and XAFS to determine the displacement of the In atoms from the GaAs (00-4)-planes, as well as the In-As strained bond lengths [2]. The InAs SQW was grown on $\operatorname{GaAs}(001)$ by MBE and capped by $25 \mathrm{~A}$ of GaAs to inhibit oxidation and to provide a bulk termination of the structure. The XSW measurements were performed at beamline X24A at the NSLS using the GaAs $(00-4)$ back-reflection geometry. and making use of the In- $L_{\alpha}$ fluorescence signal. They found $P^{H}=0.16 \pm 0.02$ and $F^{H}=$ $0.73 \pm 0.10{ }^{1}$ The corresponding In-As planar distance is equal to $1.64 \pm 0.03 \mathrm{~A}$ which agrees very well with the MET prediction of $1.62 \mathrm{~A}$ and the LDA prediction [61] of $1.63 \mathrm{~A}$.

XAFS measurements performed at the In $\mathrm{L}_{3}$-edge were used to further clarify the geometric details [2]. Even with the limited range of $k$-space available $\left(4 \rightarrow i: 2 A^{-1}\right)$ the raw data and least-squares fitting revealed a slight compression in the $\ln -A$ s bond length. with a measured value of $R_{l n-A s}^{\prime}=2.5 \tau \pm 0.02 \mathrm{~A}$ (compared with the bulk value of $R_{I n-A s}=2.623 \mathrm{~A}$ ). These measurements showed conclusively that the MET is applicable down to the ML scale, refuting the anomaly claimed by Brandt $t$ t al. [18].

An important conclusion from this work is that the principal method for strain accommodation is bond bending, rather than bond stretching [2]. This was established by comparing the energy costs of the two mechanisms. For a central atom in the zincblende lattice surrounded by four nearest-neighbours, the leading terms in the expansion of the total energy with a strain-induced distortion are

$$
E\left(\frac{\Delta R}{R}, \theta\right)=-E_{0}+4\left[\frac{1}{2} k_{R}\left(\frac{\Delta R}{R}\right)^{2}\right]+3\left[\frac{1}{2} k_{\theta}(\Delta \theta)^{2}\right] .
$$

Here $k_{R}$ and $k_{\theta}$ are the force constants for bond stretching and bending, respectively,

\footnotetext{
${ }^{1}$ Since the coherent position is only determined modula- $d_{H}$, the measured value of 0.16 is equivalent to 1.16 , to be consistent with the notation used elsewhere in this thesis.
} 
while $\Delta R$ and $\Delta \theta$ are the bond stretch and bond bend (Figure 5.2). The secondneighbour distance, $d$, in the two in-plane directions is given by $d=2 R \sin (\theta / 2)$ in terms of the nearest-neighbour bond length, $R$, and the bond angle, $\theta$. This gives

$$
\frac{\Delta d}{R}=2\left(\frac{\Delta R}{R}\right) \sin \left(\frac{\theta}{2}\right)+(\Delta \theta) \cos \left(\frac{\theta}{2}\right) \text {. }
$$

Minimizing $E$ subject to this constraint yields the bond-stretching to bond-bending ratio

$$
\frac{(\Delta R / R)}{(\Delta \theta)}=\left(\frac{3 \sqrt{2}}{2}\right)\left(\frac{k_{\theta}}{k_{R}}\right) .
$$

where the force constants are found in Reference [65]. For InAs this ratio is 0.076 . indicating that bond-bending is the dominant mechanism, in agreement with the experimental results [:].

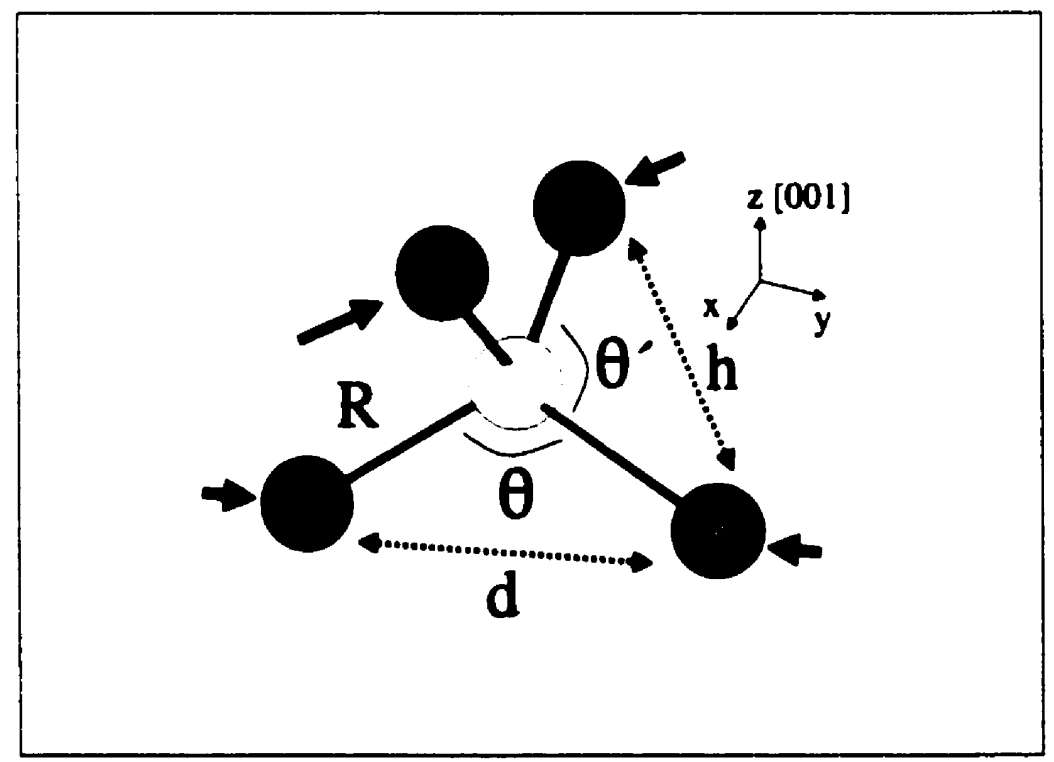

Figure 5.2: The first-neighbour environment of atoms in the zincblende lattice. In this picture the arrows indicate in-plane compressive forces which produce the observed distortions. From Reference [?]. 
Woicik et al. later compared InAs and $\operatorname{In}_{x} \mathrm{Ga}_{1-x}$ As layers buried in GaAs(001), even for InAs films beyond the critical thickness [3]. The XSW coherent positions of a $1 \mathrm{ML}$ InAs layer and a $1 \mathrm{ML} \operatorname{In}_{0.48} \mathrm{Ga}_{0.52}$ As were found to be $P^{H}=1.163 \pm 0.02$ and $P^{H}=1.053 \pm 0.02$, respectively, with coherent fractions of $F^{H}=0.72 \pm 0.1$ and $F^{H}=0.53 \pm 0.1$. The lower alloy layer position was attributed to the presence of alternating $\mathrm{In}$ and $\mathrm{Ga}$ atoms within the plane. The shorter As-Ga-As interplanar distance imposes a compressive force on the longer As-In-As interplanar distance.

For In.As layers of thickness 1.2 .4 and $8 \mathrm{ML}$, the trends in the data suggested that the measured coherent positions agreed with the strained layer predictions up to about 1.5 MLs. beyond which the layers relaxed and became disordered. With increasing InAs film thickness $F^{H}$ approached zero because the lavers differ in lattice constant from the GaAs crystal, and are each located at a different distance from the GaAs (004)-planes. This was further illustrated by the In- $\mathrm{L}_{3}$ XAFS. The 1 ML film exhibited slightly shorter first-neighbour In-As bonds than bulk InAs due to the compressive strain. For the $8 \mathrm{ML}$ film, however, the bond length reverted to its bulk value. It was found that the structural disorder due to the relaxation appeared as a reduction in ?nd and higher shell amplitudes in the XAFS [3].

The In.ts I ML sample measured with back-reflection XSW in Reference [2] was also measured using XSW at a fixed photon energy of $6 \mathrm{keV}$ by Lee et al. [4]. Their value of $P^{H}=1.154 \pm 0.01$ agrees very well with the MET prediction, and with Woicik's measurement. However, the coherent fraction was slightly lower. having the value $F^{H}=0.43 \pm 0.03$.

Lee et al. also measured the displacement between the GaAs substrate and cap using the evanescent wave effect [4]. By positioning the solid-state fluorescence detector at a shallow angle with respect to the crystal surface. and using a pair of slits to select fluorescence emission at a well-defined angle. it was possible to distinguish between signals originating from the top or bottom GaAs layers. When the XSW wavefield passes through the crystal it excites $\mathrm{Ga}$ and As fluorescence. A critical angle. $\theta_{C}$, exists for fluorescent $X$-Rays travelling out of the crystal just as for incident $\mathrm{X}$-Rays entering the crystal (although the fluorescent X-Rays have lower energy. As- $\mathrm{L}_{\alpha_{1}}=1282 \mathrm{eV}$ and $\theta_{C}=1.5^{\circ}$ ). The situation is similar to applying the discussion 
of Section 4.3.1 in reverse. So the escape depth of the $A s L_{a}$ fluorescent photons can be adjusted and used to determine the location of $A s$ atoms in the substrate and cap, relative to the substrate (004)-planes. For the substrate, the results were $P^{H}=1.00 \pm 0.01$ and $F^{H}=0.81 \pm 0.01$, obtained at a takeoff angle of $10^{\circ}$. The expected $F^{H}$ is about 0.9. corresponding to the GaAs Debye-Waller factor [34]. Measurements were then taken at smaller takeoff angles $\left(2,3\right.$ and $\left.4^{\circ}\right)$. For the cap As atoms the results were $P^{H}=0.33 \pm 0.03$ and $F^{H}=0.64 \pm 0.12$. corresponding to a $0.4 i \pm 0.04 \mathrm{~A}$ displacement of the GaAs cap relative to the (004)-substrate planes.

Recently Woicik and collaborators published several experimental [66,67,68] and theoretical [62] works aimed at resolving the outstanding issue of bond-length strain in semiconductor-alloy heterostructures. ${ }^{2}$

In K-edge EXAFS was used to determine the In-As bond lengths in a buried $\operatorname{In}_{0.22} \mathrm{Ca}_{0.78}$ As film grown coherently on GaAs $(001)$ [66]. The result of $R_{I_{n-A s}}=$ $2.581 \pm 0.004 \mathrm{~A}$ is essentially identical to the value of 2.582 .2 A predicted by a simple model based on the MET, assuming strain accommodation through equal distortions of the In-As and Cia-As bonds.

A random cluster calculation was then developed to predict bond lengths using a valence-force field [62]. Bond lengths in sixteen-bond $\mathrm{In}_{j}\left(\mathrm{Ca}_{1-}, \mathrm{As}\right.$ clusters $(j=0.1 .2 .3 .4)$ were calculated separately as a function of composition, $x$, and then statistically averaged to determine the In-.ts and Ga-As bond lengths. An illustration of the cluster appears in Figure 5.3. This calculation reproduces Mikkelsen and Boyce's bulk alloy results [54], and enabled the prediction of strained bond lengths in films grown on (001) and (111) surfaces. For the (001)-case. the predicted In-As and Ga-As strained bond lengths are contracted by nearly the same amount for a given composition, $x$, despite the inequivalent bulk bond lengths.

These predictions were verified by combining the EXAFS results of Reference [66] with diffraction anomalous fine structure (DAFS) measurements obtained at the Ga and As $i$-edges [67]. The DAFS technique makes use of constant wavevector, $\vec{q}$. measurements, $q=\|\vec{q}\|=(4 \pi / \lambda) \sin (\theta)$. Here $\lambda$ and $\theta$ refer to the $X$-Ray wavelength and

\footnotetext{
${ }^{2}$ Reference [68] reports the results of experiments performed in collaboration with SFU, and described in Section 7.9.
} 


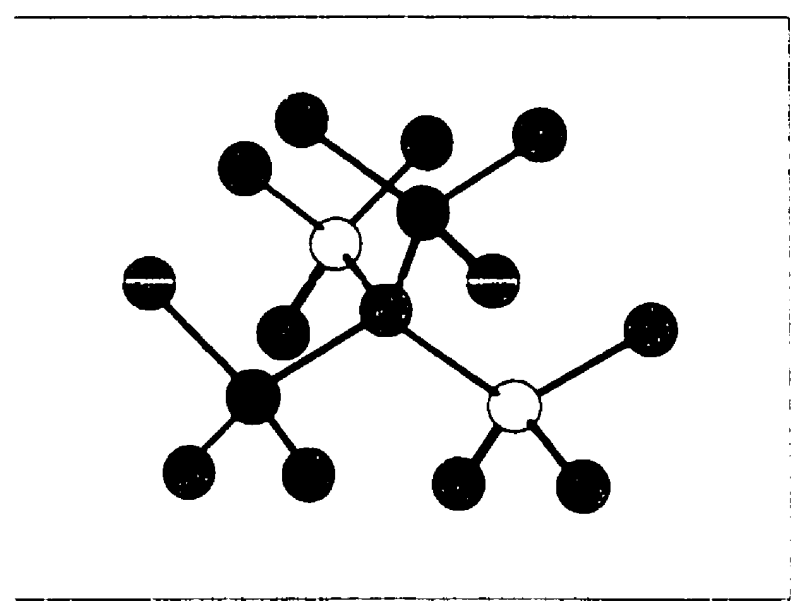

Figure 5.3: The sixteen-bond InGaAs cluster used in the model of Reference [62]. The central atom (shaded circle) is $A$ s. bonded to $j$ In atoms and $4-j$ Ga atoms (empty and solid circles), randomly occupying cation sites. The 12 peripheral $A$ s atoms of the cluster are bonded to 24 external atoms fixed at their virtual crystal positions.

the incident angle, respectively. For the $\operatorname{In}_{0.22} \mathrm{Caa}_{0.78} \mathrm{As}$ layer buried in $\mathrm{CaAs}(001)$, diffraction measurements reveal a relatively strong layer peak distinct from the substrate (004)-peak. By measuring the intensity of this peak as a function of photon energy it is possible to deduce the local environments of atoms in the laver. This technique has an advantage over conventional XAFS through the elimination of the common-element problem. XAFS at the Ga or As edges is inappropriate for this type of structure because it can not distinguish between signals originating in the strained layer or in the barrier layers. The measurements are possible using DAFS because the strained layer Bragg peak is separated from the substrate peak in reciprocal space [67]. Excellent agreement was found with the random cluster theory [62] predicting equal. compositionally-dependent distortions of the In-As and Ga-As bond lengths.

Two very recent articles by Zheng $e t$ al. have considered the dynamical diffraction occurring in structures containing $1 \mathrm{ML}$ of $\operatorname{InAs}$ buried in $\mathrm{GaAs}(001)$ [69. 70]. The first article describes a full dynamical diffraction treatment. In the second, the theory is applied to experimental measurements of samples grown by MBE, with GaAs caps 
of thickness $900 \AA$. The (004) and (220) reflections were used to determine that the In atoms were displaced by $16 \%$ from the (004)-planes. in rough agreement with the MET and XSW results [1]-[4].

\subsection{Segregation of Indium in Heteroepitaxy}

During the growth of $\operatorname{InAs}$ or $\operatorname{In}_{x} \mathrm{Ca}_{1-x}$ As layers in GaAs(001). In segregation has been observed by many researchers. While the InAs-on-GaAs surface is rather abrupt. In atoms "float" upwards with the growing surface by exchanging positions with the incoming $\mathrm{Ga}$ atoms. For single layers of InAs buried in GaAs. this process occurs during the CiaAs cap layer growtlı. Thus the In distribution is asymmetric with respect to the top and bottom interfaces. In this section the available literature will be reviewed in order to develop a complete picture of the factors influencing segregation and the techniques available for examining this process. At this time there do not appear to have been any published reports examining the segregation process in samples grown by MOVPE.

Among the first detailed segregation studies was the work by Ciuille $\epsilon t$ al. [8]. During the MBE growth of GaAs/InAs/GaAs structures, in-situ surface studies allowed a quantitative description of the segregation process. This involved both Auger Electron Spectroscopy and X-Ray Photoemission Spectroscopy (XPS) measurements of the surface In and Ga signals.

For $1 \mathrm{ML}$ of InAs deposited on GaAs the experimental results [8] showed no cation exchange at the surface. During the cap layer growth. however, significant In segregation was observed. A simple model characterized the segregation by an efficiency coefficient, $\sigma$. When the first ML of GaAs is deposited on the InAs ML (layer 1), a fraction $\sigma$ of the In atoms segregate to the surface. burying the same fraction of the deposited Ga ML. The process continues during the deposition of subsequent GaAs layers, where the composition of the $n$th layer, $\operatorname{In}_{x_{n}} \mathrm{Ga}_{1-x_{n}} \mathrm{As}$, is $x_{n}$ and

$$
x_{n}=(1-\sigma) \sigma^{n-1}
$$


It was found that the data were best described by this process with a value $\sigma=$ $0.8 \pm 0.15$.

This effect was originally attributed to the different InAs and GaAs bonding energies (1.41 $\mathrm{eV}$, and $1.59 \mathrm{eV}$, respectively), in which the more weakly-bonded atom tends towards the surface $[8]$. Strain and differences in surface reconstruction were also thought to play a role. Suppression of the segregation at higher GaAs growth rates was suggested. but not observed for rates up to $0.5 \mu \mathrm{m} / \mathrm{min}$. It was also suggested that a high GaAs cap growth temperature could be used to desorb the floating In laver. This was the first mention in the literature of "flashing-off" the segregated In atoms. Later Brandt $e t$ al. would capitalize on this idea $[i 1]$.

The same researchers later published an extensive study of segregation involving several III-As heterostructures and ternary alloys [9]. During MBE growth. RHEED. AES. XPS. Cltraviolet Photoemission Spectroscopy (UPS). and Electron Energy Loss Spectroscopy (EELS) were used to evaluate the extent of segregation, and to establish the tendencies for segregation ( $\mathrm{I} \geq \mathrm{Ga} \geq \mathrm{Al}$ ). Some samples were also studied $c s$ situ using Rutherford Backscattering (RBS). For the GaAs/InAs/GaAs system. the In segregation efficiency was again found to be near $\sigma=0.8$. independent of $T_{y}$ from $420^{\circ} \mathrm{C}$ to $560^{\circ} \mathrm{C}$, and independent of the GaAs cap growth rate.

It was noted [9] that thermodynamically, segregation at a growing surface occurs as the system attempts to balance the chemical potentials of the segregating atom in the bulk. $\mu_{b}$, and at the surface, $\mu_{s}$. This is the same mechanism driving the epitaxial process itself (Section 2.4), where the balance occurs between the chemical potentials in the (supersaturated) vapour. and in the growing solid. If the movement of a given species from the bulk to the surface yields a positive energy, $E_{s}$, then the chemical potentials in these regions are not equal for a homogeneous concentration. Instead, the surface concentration, $x_{s}$, is higher than the concentration, $x_{b}$, in the bulk.

To model the thermodynamics of the process. the balance of chemical potentials was described by McLean's equation [i2]

$$
\ln \left(\frac{x_{s}}{1-x_{s}}\right)+\frac{E_{s}}{k T_{g}}=\ln \left(\frac{x_{b}}{1-x_{b}}\right) .
$$


To account for the strain, this equation was rewritten to include the elastic energy as

$$
\ln \left(\frac{x_{s}}{1-x_{s}}\right)+\frac{E_{s}}{k T_{g}}+\frac{C^{\prime} a^{3}}{4 k T_{g}}\left(\frac{\delta a}{a}\right)^{2}\left(1+\frac{\delta a}{a}\left(x_{s}+x_{b}\right)\left(x_{s}-x_{b}\right)\right)=\ln \left(\frac{x_{b}}{1-x_{b}}\right) .
$$

where $C=\left(C_{11}^{\text {InAs }}+C_{11}^{G a s}\right) / 2$ is the mean elastic constant, a the GaAs lattice parameter, and $\delta a / a$ the lattice mismatch between InAs and GaAs. The scaling elastic factor, $C a \delta a^{2} / 4 k T_{g}$ has a value of approximately 2 for the In-Ga-As system at $527^{\circ} \mathrm{C}$. where $C^{\prime} \approx 0.6 \mathrm{eV} / \mathrm{A}^{3}$.

For high bulk concentrations and the usual low growth temperatures, the chemical potential in the bulk phase and the segregation energy are too high to be counteracted by disorder or elastic strains. and the surface concentration of the segregating species. $x_{s}$ is near unity. It was remarked that flashoff is the only way to reduce the surface concentration under these conditions [9].

To explain the observed segregation order $\left(E_{I_{n}}>E_{C_{i a}} \geq E_{A l}\right)$ it was noted that the bulk bonding energies ( InAs $1.41 \mathrm{eV}$. GaAs $1.59 \mathrm{eV}$. AlAs $1.98 \mathrm{eV}$ ) are consistent with the principle of the more weakly-bonded species segregating to the surface [9]. This arrangement did not explain the segregation order for antimonides, however (bonding energies: GaSb $1.50 \mathrm{eV}$. AlSb $1.79 \mathrm{eV})$. The bond length orders explain the results for both $\mathrm{As}$ and $\mathrm{Sb}$ compounds. where $R_{I n-A s}(2.62 \mathrm{~A})>R_{G u-A s}(2.45 \mathrm{~A}) \approx R_{A l-A s}$ $(2.45 \mathrm{~A}):$ and $R_{G a-S b}(2.64 \hat{A})>R_{A 1-S b}(2.66 \mathrm{~A})$.

Predictions of the segregating species were not [9] expected to be straightforward. and it was noted that the order could be reversed for different crystal orientations. Indeed. He et al. recently observed Ga segregation for InAs growth on $\operatorname{GaAs}(110)$ using soft XPS [73]. Other factors such as the surface reconstruction or relaxation. not taken into account by the modified McLean equation, were also expected to be involved in the process.

Since the cation exchange reactions are rapid with respect to the growth, segregation was not supposed to be kinetically limited under usual growth conditions [9]. Reducing segregation by manipulation of the kinetics was suggested, and could be effected by decreasing the migration rates of the atoms at the interface, the number of reaction sites, and/or the rate of reaction at these sites.

According to the thermodynamic model, decreasing $T_{g}$ favours segregation but 
decreases the surface migration [9]. Thus alternating flux epitaxies (ALE or its MBE counterpart, migration-enhanced epitaxy (MEE)) seem to be unsuitable for producing abrupt interfaces because of the high cation surface mobilities. unimpeded by excess anion flux. It was suggested that growth of the GaAs overlayer using a low-surfacemobility technique would result in sharper interfaces.

Observations of In segregation have been made using RHEED oscillations [it] during chemical beam epitaxy (CBE) of InGaAs, as well as in MEE [75]. In the latter. characteristic oscillations in the RHEED intensity were observed during the alternating $\mathrm{Ga}$ and As fluxes of the MEE cycle. When a single In pulse was substituted for a Cia pulse, a marked decrease in the RHEED amplitude occurred for growth temperatures of 400,500 and $560^{\circ} \mathrm{C}$. When the CiaAs capping cycles were initiated the surface recovered to its original state for growth at 400 and $560^{\circ} \mathrm{C}$. but not at $500{ }^{\circ} \mathrm{C}$. Secondary ion mass spectroscopy (SIMS) suggested that the incorporated In was less than expected for the $560^{\circ} \mathrm{C}$ sample, indicating In evaporation. The In SIMS profile was found to be asymmetric for the $500^{\circ} \mathrm{C}$ sample, but symmetric for the others. This suggests that the In segregation increases with increasing $T_{g}$. until higher temperatures at which the desorption removes the segregating atoms. The segregation was attributed to the difference in InAs and GaAs bonding energies. although previous work suggested otherwise [9].

The "flashoff" technique mentioned earlier was realized by Brandt et al [10]. who studied the structural and optical properties of their films using HRXRD, doublecrystal X-Ray reflection topography. HREM, PL and PL excitation (PLE). Buried layers of InAs in $\operatorname{GaAs}(001)$ were deposited by MBE using the following sequence. A $1 \mu \mathrm{m}$ GaAs buffer was deposited at $540^{\circ} \mathrm{C}$, followed by the InAs layer growth at $420^{\circ} \mathrm{C}$. A few ML of GaAs were then deposited at $420^{\circ} \mathrm{C}$, after which a thicker GaAs cap was grown at $540^{\circ} \mathrm{C}$. The hypothesis was that any segregated In would be contained within (at worst) the first few cap MLs, and that heating to $540^{\circ} \mathrm{C}$ desorbs the excess In. The term "flashoff" was coined later [71].

HRXRD data were interpreted using a semi-kinematical description [55] assuming coherency of the InAs layer with the substrate, and strain accommodation by an elastic deformation of the InAs unit cell [10]. The simulations suggested an InAs 
layer thickness of 1.2 ML and X-Ray reflection topographs confirmed the absence of structural dislocations. The InAs-on-GaAs growth rate was only $64 \%$ of the bulk InAs growth rate because the heteroepitaxial strain acts as a kinetic barrier to the incorporation of In atoms in the crystal. This indicates the complexity of the growth process at the level required for characterizing these ultrathin films.

A review by Moison $e t$ al. [i6] including results of surface studies, HREM. RBS. PL and Raman scattering drew the same conclusions as the earlier work. ['sing the thermodynamic prediction of reduced segregation at elevated temperatures, it was noted that the required temperatures are too high $\left(>1000^{\circ} \mathrm{C}\right)$ to be suitable. Reducing segregation by slowing down the kinetics was suggested. though it was thought that GaAs growth rates could not be increased enough to bury the In atoms before their migration to equilibrium positions on the surface.

Brandt et al. discussed the details of the "flashoff" technique in a later work [i1]. Due to the apparent independence of segregation on growth parameters (temperature and material fluxes) for typical MBE procedures. the flashoff sequence was suggested as a means of combatting the kinetic and thermodynamic barriers to the formation of abrupt interfaces. Their InAs laver was deposited in increments of 0.6 ML. annealing the surface under $\mathrm{As}_{4}$ for $120 \mathrm{~s}$ at $420^{\circ} \mathrm{C}$ after each deposition. The capping scheme was similar to that described earlier [10] where the thickness of the low $T_{g}$ cap is an essential parameter. Structures grown in this way were evaluated by RHEED in-situ. HRXRD and HREM.

RHEED patterns obtained during the deposition of the InAs layer and GaAs cap were used [il] to study the evolution of the surface reconstructions. When the temperature was lowered for InAs growth. the GaAs $(2 \times 4)$ reconstruction changed to the $c(4 \times 4)$, due to excess $A s_{4}$ adsorption. After the first incremental InAs deposition a $(2 \times 3)$ pattern emerged, corresponding to $\mathrm{As}_{\mathbf{4}}$-stable conditions. Since the impinging fluxes favoured In-rich conditions. the As termination was attributed not to the impinging $\mathrm{As}_{4}$ flux. but to the excess As already present on the GaAs surface. After the second increment the reconstruction streaks disappeared. but developed again during the growth interruption. Thus the growth interruptions apparently played an important role in allowing the dynamic recovery toward an equilibrium surface state. When 
the GaAs cap growth was initiated a $(1 \times 1)$ pattern was observed. due to the floating In layer. Raising the temperature produced a well-defined $(2 \times 4)_{r}$ reconstruction. apparently related to the flashoff of the floating In surface layer. The In incorporation in flashed-off samples was estimated to be $40 \%$ less In than in films without the flashoff. while the amount of segregated In was found to be insensitive to the growth conditions [i1]. An exception appeared in samples grown by . MEE with segregation rates of up to $90 \%$.

Structural quality in the flashoff films was evaluated by HRXRD [i1]. Buried InAs SQWs of thickness 3.6 ML and SLs with 0.8 ML of InAs were produced with low $T_{g}$ for the initial 3 MLs of the GaAs cap layer growth. Symmetric and asymmetric HRARD data were modelled using Tapfer's approach and assuming pseudomorphism with the CiaAs substrate. The agreement between the data and the theoretical models up to the 11th-order superlattice satellites was at tributed to the Inds laver perfection. For the InAs SQW it was found that the InAs thickness remaining after the flashoff was only 2.4 ML, indicating that $1 / 3$ of the amount deposited was desorbed in the flashoff. HREM images of the heterointerfaces were presented as evidence of the atomic scale interfacial abruptness. and of the structural equivalence of the top and bottom interfaces [i1].

Gérard et al. used an optical approach to study the segregation effect in In As layers deposited by $\mathrm{MBE}$ at $540^{\circ} \mathrm{C}$ within $\mathrm{GaAs} / \mathrm{Al}_{x} \mathrm{Ga}_{1-s}$ As quantum wells [i $\left.i\right]$. The idea was that the PL spectra would be affected by the position of the InAs layer, either close to the bottom or the top $\mathrm{GaAs} / \mathrm{Al}_{x} \mathrm{Ga}_{1-x}$ As interface. Segregation would spread the In distribution towards the top interface. leading to emission energies different from the QW with the InAs deposited closer to the bottom interface. Observed differences in the emission energies for $Q W$ s of this type were interpreted using the model of Equation 5.9 yielding a segregation energy, $E_{s}$, in the range of $0.15-0.20 \mathrm{eV}$. in agreement with Reference [9].

Hansen et al. studied the bulk and near-surface composition of $\operatorname{In}_{x} \mathrm{Ga}_{1-x}$ As epilayers of $0.5-1.0 \mu \mathrm{m}$ thicknesses (fully relaxed), grown on $\mathrm{GaAs}(001)$ by CBE at temperatures from $470 \rightarrow 600{ }^{\circ} \mathrm{C}$, and with different TEIn flow rates [i8]. In-situ. 
the bulk compositions were determined from the change in RHEED oscillation periods. The bulk In content was determined by ex-situ XRD. while XPS was used to evaluate the near-surface composition profiles. Comparing the $x$-values deduced from XRD and from Fourier analysis of the RHEED oscillations, it was found that RHEED consistently underestimates the mole fraction. The data obtained by both methods were highly correlated at $470{ }^{\circ} \mathrm{C}$, but not for higher temperatures. This discrepancy was associated with competitive incorporation of TEGa and TEIn. At the high temperatures surface desorption was thought to play a role in the surface kinetics. The underestimation of the In fraction could be explained by the fact that the RHEED transients were measured in the early, pseudomorphic stages of growth. while the XRD data were obtained following the relaxation of the layer. XPS analysis showed little segregation occurring at $470^{\circ} \mathrm{C}$. At higher temperatures. analysis of the Ga $3 p$ and In 3p peaks showed Ga surface depletion accompanied by an In enrichment extending $50-75 A$ into the samples.

Using flow-rate modulation epitaxy (FME), in which an alternating supply of organometallic alkyls and arsine were transported to the reactor. Sato $\mathrm{et}$ al. prepared pairs of InAs SQW' in GaAs [79]. FME differs from ALE in that small amounts of the group $V$ source accompany the group III flow to the reactor. A theoretical model was derived to include segregation effects in the prediction of PL emission energies. For FME samples with different GaAs spacings between the two InAs layers, the PL energies were well described without In segregation. It was suggested [79] that dangling bonds of the surface $A$ s atoms were terminated by $\mathrm{H}$ atoms which prevented the In/Ga exchange. In contrast, when this model was applied to the experimental results of Gérard et al. [iٓ] for MBE films, a segregation efficiency of $90 \%$ was found.

The influence of In segregation on quantized energy levels in InGaAs/GaAs QWs was studied by Muraki et al. using SIMS and PL [80]. Growth condition effects were studied by preparing a series of samples with constant well widths and different growth temperatures, or constant $T_{g}$ and different well widths. It was found that the $1 / e$ decay length of the In SIMS signal increased from $8 \AA$ to $29 \hat{A}$ when $T_{g}$ was increased from 370 to $520^{\circ} \mathrm{C}$. Although the segregation was reduced for growth below $420{ }^{\circ} \mathrm{C}$, the PL intensities were lower due to the poor crystalline quality of the material. The 
best PL emission was obtained in the samples grown at $520^{\circ} \mathrm{C}$. A model for the PL emission energies showed that including the segregation-related compositional grading was necessary to reproduce the experimental transition energies. The segregation was thought to be kinetically limited at the lowest growth temperatures, increasing with increasing temperature, and reduced above $570^{\circ} \mathrm{C}$ due to In desorption.

SIMS was also used by Lawai et al. to study the spread of the In distribution in MBE samples grown from $400 \rightarrow 530^{\circ} \mathrm{C}$ [81]. The samples were prepared by growing a GaAs buffer layer at $580^{\circ} \mathrm{C}$ and then allowing the substrate to equilibrate at the desired $T_{y}$. A single InAs ML was then deposited, followed by $2000 \mathrm{~A}$ of GaAs at the same temperature. It was found that the In spreading width observed with SIMS was present even at $400{ }^{\circ} \mathrm{C}$ and increased with increasing $T_{y}$. Thicker InAs lavers were also prepared. and suggested that the segregation was markedly increased when dislocations were generated near the interface.

Increasing segregation with increasing $T_{g}$ was also observed in RHEED and ('PS studies of InCiaAs/CiaAs samples prepared by MBE between 490 and $58: 5^{\circ}\left(\mathrm{C}^{\circ}\left[\mathrm{8}^{\circ} 2.833\right.\right.$. The segregation was apparently also reduced at higher V/III ratios. PL spectra for these samples were modelled using a stepwise approximation to the segregated In profiles yielding good agreement with the experimental emission energies. This involved the use of Equation 5.8 with a segregation energy of $E_{s}=0.22 \mathrm{eV}$. The temperature dependence of this equation was not discussed. Throughout the growth sequence the following evolution of the RHEED pattern was observed [82]: $(2 \times 4)$ during the GaAs buffer layer. $(1 \times 1)$ during the InGaAs. $(1 \times 1)$ during the beginning of the Ga.As overlayer, and $(2 \times 4)$ after $\sim 12$ ML. It was noted that enhanced segregation was associated with a delayed recovery to the $(2 \times 4)$-reconstruction. When the samples were cooled under $A$ s pressure, the $(1 \times 1)$ and $(2 \times 4)$ patterns were transformed into $(1 \times 3)$ and $c(4 \times 4)$, respectively.

The conflicting requirements for minimizing the effects of interface roughness and surface segregation were pointed out by Bosacchi et al. [84]. While segregation could be reduced at lower $T_{g}$, the roughness is minimized at higher temperatures which. unfortunately, enhance the cation migration at the growth front. Using conventional MBE and atomic layer MBE (ALMBE) to produce a series of InGaAs MQWs and 
InAs SQWs in GaAs, the segregation was studied using PL. SQW samples were prepared by growing thick $(0.9 \mu \mathrm{m}) \mathrm{GaAs}$ buffer layers at $600{ }^{\circ} \mathrm{C}$ and then decreasing the temperature. Thinner GaAs barriers $(0.1 \mu \mathrm{m})$ were deposited at different temperatures between 300 and $495{ }^{\circ} \mathrm{C}$. followed by the growth of the InAs MLs at the same temperature and a growth rate of $0.06 \mu \mathrm{m} / \mathrm{hr}$. For the $\mathrm{MBE}$ samples, the temperatures were linearly ramped to $600^{\circ} \mathrm{C}$ during the growth of the Ga.As cap layers. ALMBE caps were deposited at the same temperature as the Ints layers and with growth rates of $1.3 \mu \mathrm{m} / \mathrm{hr}$.

PL spectra obtained at $10 \mathrm{~K}$ revealed redshifts of the enission energies with decreasing $T_{g}[84]$ and saturation of the transition energies at minima corresponding to minima of In surface segregation. ALMBE samples exhibited enhanced segregation effects, evidenced by the higher transition energies compared with the MBE samples. This was attributed to the greater exchange reaction rates during the ALMBE groupIII sub-cycles. In the absence of an accompanying As flux. the Cia can more easily exchange positions with previously adsorbed In atoms. Saturation of the PL energies was observed at growth temperatures of 260 and $340^{\circ} \mathrm{C}$ for the AL.MBE and MBE samples. respectively. The PL energy curves werc found to be concave up with respect to $T_{y}$.

The temperature dependence of the thermodynamic model (Equations 5.8 or 5.9) was considered by Dehaese et al. [85]. An increase in segregation with decreasing growth temperature is predicted because of the term $\exp \left(E_{s} / k T_{g}\right)$. As the preceeding discussion clearly shows. this is not consistent with the available experimental data. A kinetic model was developed to address this issue for InGaAs growth following work for dopant incorporation and segregation in MBE growth [86, 87. 88]. The following discussion will adopt the notation of Harris et al. who studied $\mathrm{Sn}$ incorporation and segregation in MBE-grown GaAs. The case of In segregation in GaAs is analogous.

A schematic representation of the $\mathrm{In} / \mathrm{Ga}$ exchange process is reproduced in Figure 5.4. where $E$ is the free energy of the system. The distance between the energy minima is roughly equal to the ML thickness and $E_{s}$ is the segregation energy of the equilibrium model.

Suppose that the initial surface coverage of In is $N_{0}$ atoms $/ \mathrm{cm}^{-2}$, and that the 


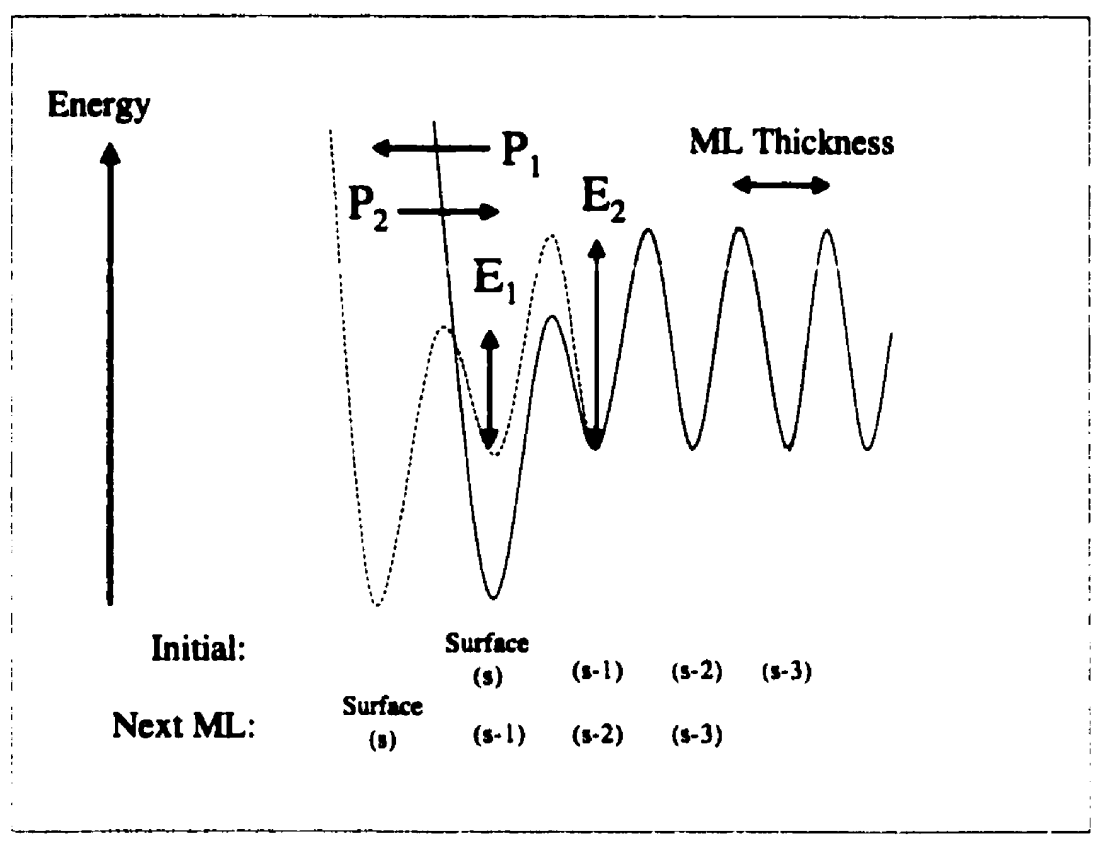

Figure 5.4: Schematic representation of the energy of an $\ln$ atom as a function of depth within a GaAs film. Each minimum corresponds to a cation plane, with spacing $a_{G a A s} / 2$ in the (001)-direction. The solid line represents the initial situation and the dashed line shows the situation after the deposition of an additional GaAs ML. From Reference [87].

InAs layer has been capped by a single GaAs ML. In Figure 5.4. $E_{1}$ is the energy barrier for segregation and $E_{2}$ is the barrier for the reverse process. The transition probabilities for the two processes are $P_{1}$ and $P_{2}$, respectively. After 1 capping ML the original In surface becomes layer $(s-1)$, while $s$ is the new surface. The time evolution of the number of atoms per unit area in layer $s-1, N_{s-1}$, is

$$
\frac{d N_{s-1}}{d t}=-P_{1} N_{s-1}+P_{2} N_{s}=P_{1}\left(\phi N_{s}-N_{s-1}\right) .
$$

where $\phi=P_{2} / P_{1}$. Since $N_{s}+N_{s-1}=N_{0}$, this becomes

$$
\frac{d N_{s-1}}{d t}=P_{1}\left[\phi N_{0}-(1+\phi) N_{s-1}\right] \text {, }
$$


which has solution

$$
N_{s-1}=\frac{N_{0}}{(1+\phi)}\left(\phi+\exp \left[-P_{1}(1+\phi) t\right]\right) .
$$

making use of the BC $N_{s-1}=N_{0}$ at $t=0$ (the In atoms are completely buried for a brief instant).

The equilibrium siluation may be considered in the linit as $l \rightarrow \infty[8]]$. Then $N_{s-1}=N_{0} \phi /(1+\phi)$ and $N_{s}=N_{0} /(1+\phi)$. In thermal equilibrium.

$$
\frac{N_{s-1}}{N_{s}}=\phi(t \rightarrow \infty) \text {. }
$$

The quantity $\omega$ is related to the free energy difference which drives the segregation

$$
0=\operatorname{texp}\left(\frac{-E_{s}}{k \cdot T_{y}}\right) \text {. }
$$

where $f$ contains the vibrational frequency and other factors related to the hopping probabilities [8T].

Consider GaAs cap growth at a rate 1 ML per $\tau$ seconds. If the next Cia-As ML growth starts before the In atoms reach their equilibrium distribution. then there is a kinetic limitation to the segregation process. This will dominate when the segregation is slow compared with the ML deposition rate: low $T_{g}$ or high growth rates. In contrast, at high temperatures or low growth rates the kinetic effects will be small and the incorporation process will tend towards the equilibrium segregation limit. Harris et al. derived an equation for the incorporation constant. $k_{*}$, which is valid over all temperature and growth rate regimes [8T].

If $1 \mathrm{ML}$ of GaAs, thickness $a_{G a A s} / 2$, is deposited in a time $\tau$, then

$$
N_{s}(\tau)=\frac{N_{0}}{(1+\phi)}\left(1-\exp \left[-P_{1}(1+\phi) \tau\right]\right)=N_{0} \sigma,
$$

where $\sigma$ is the segregation efficiency as in equation 5.7. After depositing $q$ MLs. the In coverage of the original surface layer, $(s-q)$, will be $N_{0}(1-\sigma)$. For subsequent layers

$$
\begin{array}{ll}
(s-q+1) & \rightarrow N_{0} \sigma(1-\sigma) \\
(s-q+2) & \rightarrow N_{0} \sigma^{2}(1-\sigma) \\
(s-1) & \rightarrow N_{0} \sigma^{(q-1)}(1-\sigma) \\
s & \rightarrow N_{0} \sigma^{q}
\end{array}
$$


This is an exponentially decaying distribution up to laver $s-1$, equivalent to the distribution described by equation 5.T. If the In distribution along the $z$-growth direction has the form [si]

$$
n(z)=n_{0} \exp \left(-k_{z} z\right)
$$

then we note that since

$$
N_{s-1}=N_{s-q} e x p[(q-1) l n \sigma] .
$$

and $z=(q-1) a_{G a A s} / 2$, the decay parameter has the form

$$
k_{:}=\frac{-1}{(q-1) a_{G a . t s} / 2} \ln \left(N_{s-1} / N_{s-q}\right)=\frac{-1}{a_{G i \text { is }} / 2} \ln \sigma .
$$

Using Equation 5.15.

$$
\left(\frac{a_{C i a A s}}{2}\right) k_{z}=\ln (1+\phi)-\ln \left[1-\epsilon x p\left[-P_{1}(1+\phi) \tau\right]\right] .
$$

For high $T_{g}$ and relatively slow growth rates $(\tau \operatorname{large})$ the first term on the right dominates and

$$
\left(\frac{a_{G u t \cdot s}}{2}\right) k: \approx 0 \equiv \exp \left(-E_{s} / k T_{y}\right) .
$$

For low $T_{g}$ and fast growth rates it is assumed that the transition from $s$ to $s-1$ proceeds according to

$$
P_{1}=P_{0} \exp \left(-E_{1} / k T_{y}\right) .
$$

corresponding to a barrier of height $E_{1}$. This gives

$$
\ln \left(k_{z} a_{G a A s} / 2\right)=-P_{0} \tau \exp \left(-E_{1} / k T_{g}\right) .
$$

This model was shown to describe MBE results for Sn segregation both in the high$T_{g}$ regime. and at low temperatures where kinetic barriers prevent the equilibrium surface concentration from being achieved [8i]. However, the model neglects any dependence of the segregation on the As flux or on the incoming V/III ratio. It was pointed out [87] that the important parameters may not be the incident fluxes so much as the surface concentrations of the various species, which are difficult to measure. The segregation is likely to be influenced by adatom populations in either chemisorbed or precursor states which may not be directly controlled. 
In the formulation of Reference [85] it was found that the kinetic limitation of segregation for InGaAs growth occurs below $400^{\circ} \mathrm{C}$, and for growth rates in excess of about $1 \mathrm{ML} / \mathrm{s}$.

It is interesting to note that the model of Reference [8T] shows two distinct regimes. Williams et al. [89] observed this type of behaviour using in-situ UPS and RHEED during the growth of GaAs/InGaAs/GaAs(001) structures by MBE. Following the deposition of $5-10 \mathrm{ML}$ of $\mathrm{In}_{0.30} \mathrm{Ga}_{0.70}$. As a rapid increase in the In surface concentration was observed with increasing temperature during the GaAs cap layer growth. An Arrhenius plot showed that the In segregation consisted of two components. Below $500^{\circ} \mathrm{C}$ a "fast" component was observed with an activation energy of $0.18 \mathrm{el}$. Above $500^{\circ} \mathrm{C}$ a "slow" component with activation energy of $2.49 \mathrm{eV}$ was observed, accompanied by a change in the surface reconstruction. The InGiats surfaces displayed $(1 \times 3)$ reconstructions. while the CiaAs cap surfaces showed $(2 \times 2)$ patterns at lower temperatures. At higher temperatures, the $(2 \times 2)$ surfaces did not appear. suggesting that a significant amount of In remained on the surface [89]. It was noted that the slow component could be due to the generation of misfit dislocations.

Arès et al used RDS to study the evolution of surface anisotropies during the ALE growth of GaAs/InAs/GaAs(001) structures. as well as the reverse "antiwell" (InAs/GaAs/InAs(001)) structures [90]. During a typical ALE cycle for the growth of $1 \mathrm{ML}$ of material the $2.56 \mathrm{eV}$ RDS transient exhibits a characteristic pattern. During the growth of several MLs the signal is periodic and highly reproducible from cycleto-cycle. When a single In pulse was substituted for a Ga pulse in the sequence. the RDS signal became strongly positive, and the shape of the individual cycles was strongly modified during the growth of the following GaAs cap layers. The recovery of the unperturbed GaAs cycle signal required more than $20 \mathrm{ML}$ of growth, indicating a high degree of segregation.

In contrast, during the antiwell growth, the InAs cycle signal was recovered in less than 2 ML [90]. This complementary experiment was particularly useful in that it demonstrated that (i) the slow recovery of the GaAs signal in the QW growth was not the result of reactor memory effects, (ii) the slow recovery was not due to a buried anisotropy associated with the heteroepitaxy, and (iii) as expected, In has a greater 
tendency for segregation on the (001) surface.

In the segregation models previously discussed the segregation energy. $E_{s}$, cannot be evaluated a priori from the growth characteristics. A Monte Carlo simulation for InGaAs growth on GaAs was developed by Grandjean et al. which accounts for the binding energies of the constituents. the epitaxial strain and the growth parameters [91]. It was found that the experimental PL emission energies were well-described by the simulation results with the relevant growth parameters.

In the simulations the "hopping rate". $h$, was defined as $h=h_{0} \exp \left(-E_{d} / k T_{y}\right)$ (c.f. Equation 5.21). where $h_{0}=k T_{g} / \pi \hbar$ is the adatom vibration frequency $\left(h_{0}=\right.$ $3.2 \times 10^{13} s^{-1}$ at $\left.500{ }^{\circ} \mathrm{C}\right)$. Here $E_{d}$ is the energy barrier to surface diffusion which consists of a substrate contribution, $E$ : a contribution due to the number of nearest neighbours in the $(110)$ and $(\overline{1} 10)$ directions, $p E_{(110)}+q E_{(\overline{1} 10)}$ : and an elastic energy contribution. $E_{e}$ which opposes the other factors. The quantities $E_{(110)}$ and $E_{(110)}$ are the axis-dependent bonding energies with the nearest neighbours $(p . q \sim 0.2)$. A ratio of $E_{(110)} / E_{(110)} \sim 6$ was used in the calculation [91]. The elastic term was calculated from $E_{e}=\left(1-a_{n}\right) E_{e}^{(n=4)}$. where $E_{e}^{(n=4)}=1: 34 \mathrm{mel}$ is the elastic energy for a tetragonal distortion with + nearest neighbours and the coefficient $\alpha_{n}$ accounts for free surfaces with dangling bonds.

The model developed in this way was found to provide good agreement with experimental PL energies, and showed the importance of including the elastic strain [91]. It was demonstrated that increasing the growth rate or decreasing $T_{g}$ could be used to kinetically limit the segregation process.

Segregation effects on the optical properties of MBE-grown QWs were later studied by the same researchers in collaboration with Disseix et al. [92]. The kinetic approach of Reference [85] was used to analyze the thermally detected optical absorption (TDOA) data obtained for InGaAs/GaAs(001) structures prepared under various conditions of temperature and growth rate. In the TDOA technique the heating of the sample caused by phonon emission due to nonradiative deexcitation, which occurs after optical absorption, is detected by a thermometer maintained with the sample at low temperature $\left(0.35 \mathrm{~K}\right.$ was obtained in a ${ }^{3} \mathrm{He}$ refrigerator).

In the data analysis, the kinetic model was used to describe the In segregation [92]. 
These results were incorporated into an effective-mass model for the confinement energies in the QWs. Experimental data indicated that the effect of segregation was to increase the transition energies, and that a kinetic limitation is effected at low $T_{g}$. Fitting the data yielded a segregation energy of $E_{s}=0.13 \pm 0.01 \mathrm{eV}$. It was noted that decreasing $T_{g}$ by $50^{\circ} \mathrm{C}$ at a fixed growth rate of $0.5 \mathrm{ML} / \mathrm{s}$ had more of an effect on the segregation than an increase in the growth rate from $0.5 \mathrm{ML} / \mathrm{s}$ to $1.3 \mathrm{ML} / \mathrm{s}$ at $500{ }^{\circ} \mathrm{C}$.

Yamaguchi et al. also employed the kinetic segregation modei to describe data obtained by SIMS and PL for InGaAs/GaAs(001) samples prepared under various MBE growth conditions [93]. Growth temperatures of between 430 and $500{ }^{\circ} \mathrm{C}$ were used. with growth rates between 0.2 and $1.6 \mathrm{ML} / \mathrm{s}$, and $V / I I I$ ratios between 8 and $2 \pi$. Increasing segregation was observed with increasing $T_{g}$, which saturated at about $5.50{ }^{\circ} \mathrm{C}$. This was attributed to the convergence of the kinetic and equilibrium descriptions of the segregation process at higher temperatures. PL results indicated an increase in segregation with decreasing cap growth rate as seen in the PL blueshifts. Although the As flux was not included in the kinetic model. it was found experimentally that the segregation was enhanced by low As pressures which produced In-stabilized surfaces. The segregation energy was found to be about $0.10 \mathrm{eV}$ for growth under As-stabilized conditions.

\subsection{Reflectance Difference Spectroscopy}

For characterization of growing surfaces during MOVPE, the optical techniques of RDS and ellipsometry have found widespread use, since electron-beam techniques such as RHEED are prohibited in non-UHV environments [94]. RDS measures the difference between the normal-incidence reflectances, $\dot{r}_{\overline{1} 10}$ and $\dot{r}_{110}$, for light polarized along the [110] and [110] principal axes of the (001) surface [95]. This quantity is determined experimentally as a function of photon energy and growth conditions. Bulk contributions dominating each of the reflectances are nearly identical and cancel in subtraction, leaving a difference related to the surface anisotropy. This surfacesensitivity is the major strength of RDS. 
RDS measures the optical anisotropy of III- $V$ crystals through the quantity

$$
\frac{\Delta \tilde{r}}{\dot{r}}=\frac{2\left(\dot{r}_{110}-\dot{r}_{110}\right)}{\left(\tilde{r}_{110}+\dot{r}_{110}\right)}=\frac{\Delta r}{r}+i \Delta \theta \text {. }
$$

An example of a RDS spectrum obtained in the SFC system is shown in Figure 5.j. The data presented in this thesis show the real part of the RDS.

$$
\frac{\Delta r}{r}=2 \operatorname{Re}\left[\frac{\dot{r}_{110}-\dot{r}_{110}}{\dot{r}_{110}+\dot{r}_{110}}\right] \text {. }
$$

which is primarily related to the imaginary part of the dielectric function [96].

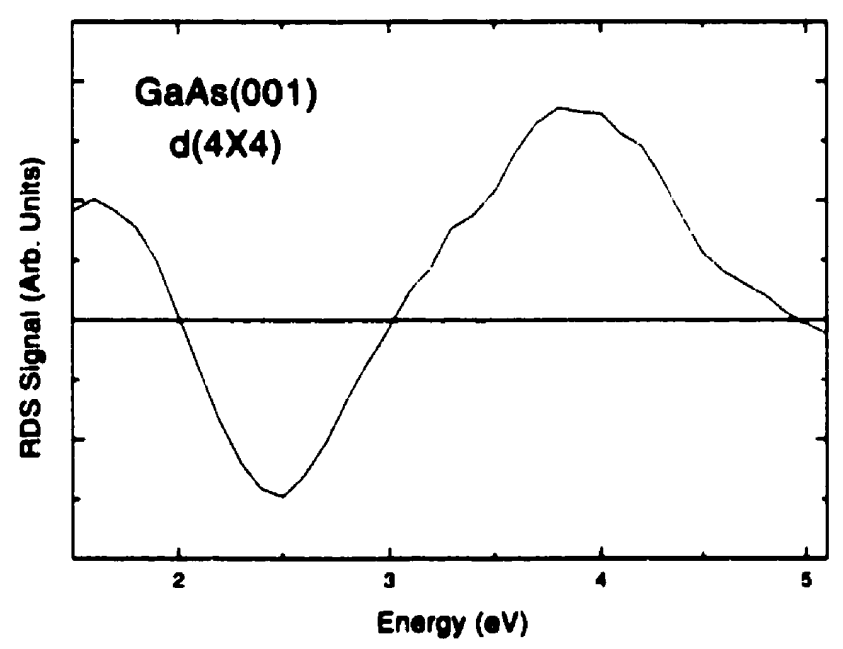

Figure 5.5: An RDS spectrum of the GaAs( 001$) \mathrm{d}(4 \times 4)$ reconstruction (disordered $\mathrm{C}(4 \times 4))$. The spectrum was obtained at $550{ }^{\circ} \mathrm{C}$ and 50 Torr under TBAs.

Early on, a "database" was established for GaAs surfaces by associating RDS spectra with known surface reconstructions determined by RHEED [97]. This has allowed subsequent work to refer directly to these data. Figure 5.6 shows two common reconstructions of the $\mathrm{GaAs}(001)$ surface. The oscillating electric field of the linearlypolarized light causes transitions related to dimers oriented along the principal axes of the crystal. The major features of the RDS spectra may be related to these electronic 
transitions. For transient RDS spectroscopy on $\mathrm{GaAs}(001)$ it is often useful to monitor the RDS signal near $2.5 \mathrm{eV}$, which is associated with As dimers on the surface and is particularly sensitive to the nature of the reconstruction. In Figure 5.5 the minimum at $2.5 \mathrm{eV}$ is due to As dimers oriented along [110]. The $\mathrm{d}(4 \times 4)$ reconstruction is formed by adding excess $A s$ to $c(4 \times 4)$ and is distinguishable from the $c(4 \times 4)$ by the RDS peak at $4.0 \mathrm{eV}[97]$.

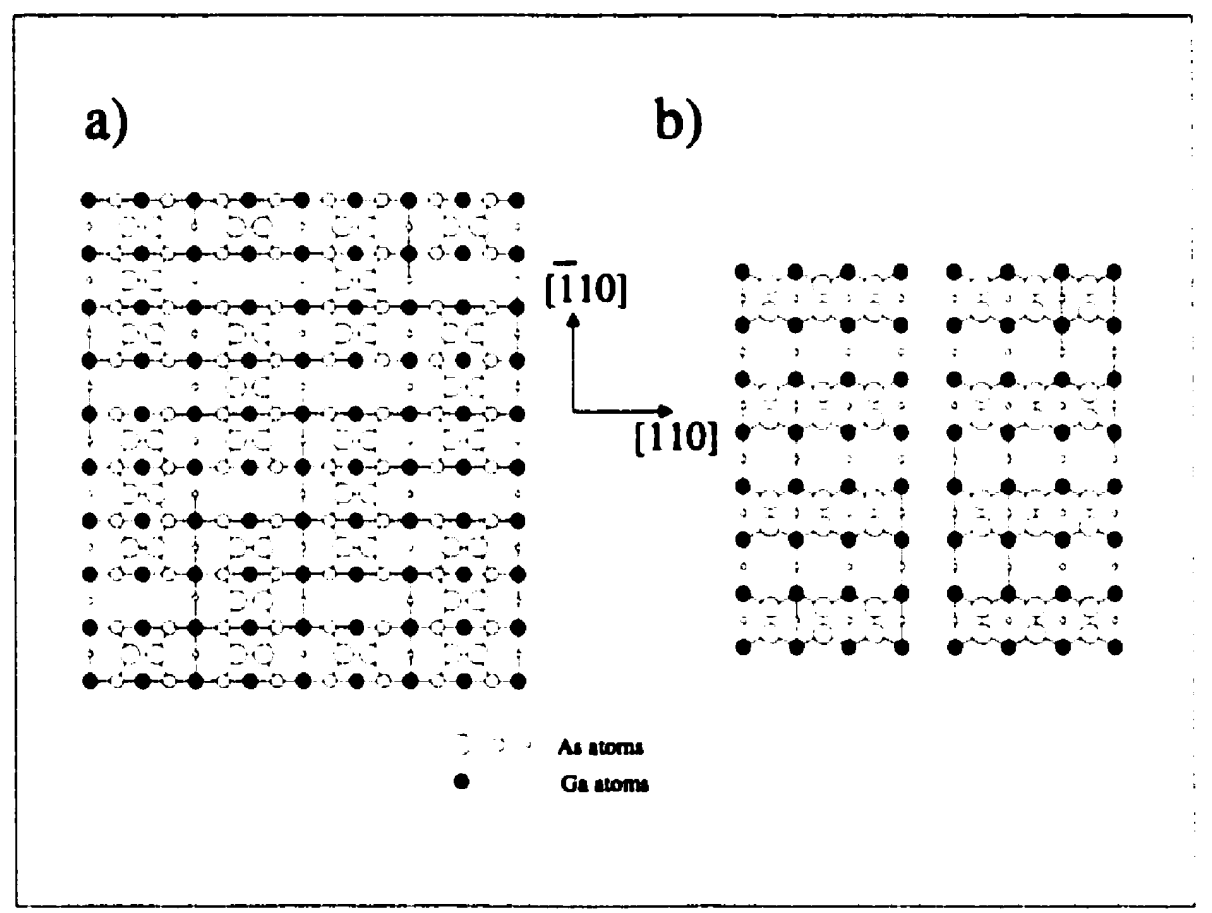

Figure 5.6: Models of the a) $c(4 \times 4)$ and b) $(2 \times 4)$ reconstructions of CiaAs $(001)$. Larger circles indicate atoms closer to the surface. From Reference [98].

Kamiya et al. studied the $\mathrm{GaAs}(001)$ surface extensively using RDS and identified reconstructions in atmospheric pressure environments $\left(\mathrm{H}_{2}, \mathrm{He}\right.$ and $\left.\mathrm{N}_{2}\right)$ similar to those observed in $\mathrm{UHV}[9 \tau, 98,99]$. The temperature dependence of the RDS spectra was similarly explored. Additionally, the RDS and RHEED data obtained under dynamic surface conditions clearly showed a correspondence between the two 
techniques, establishing RDS as a suitable tool for controlling epitaxial growth [95].

In the MOVPE environment. Aspnes et al. studied processes occurring during ALE growth using transient spectroscopy at $2.5 \mathrm{eV}[100]$. A novel technique was later developed to allow a complete real-time investigation of the surface evolution during ALE growth [101]. The technique exploits the reproducibility of individual ALE cycles to obtain time-resolved data with very high temporal resolution. Multitransient spectroscopy was perfomed by acquiring a set of transient spectra. each obtained at a fixed energy from 1.5 to $5.5 \mathrm{eV}$ in steps of $0.5 \mathrm{eV}$. with each transient synchronized to the same instant of the exposure cycle. The data were then reorganized to form a series of energy spectra. each corresponding to a specific instant in the ALE cycle. This technique was applied in this thesis to allow the observation of the surface anisotropy development during $\mathrm{SQW}$ growth sequences at a range of growth temperatures (Section 7.6.1).

Further evidence of the sensitivity of RDS to surface reconstructions was obtained in simultaneous measurements using RDS and grazing incidence X-Ray scattering (GINS) performed at SSRL [102, 103]. MOVPE growth was performed in a speciallydesigned vertical reactor [104] which allows the in-situ GIXS measurements. The RDS spectrum of the GaAs(00l) under TBAs was found to be virtually identical to Figure 5.5, which was obtained in a similar reactor under similar conditions. GIXS measurements made along the $[110]$ and $[\overline{1} 10]$ azimuths revealed distinct peaks associated with the $c(4 \times 4)$. A Ga-rich $(4 \times 6)$ reconstruction was observed upon exposing the sample to TEGa. By varying the measurement conditions slightly it was possible to produce the $(4 \times 6),(2 \times 4)$ and $(4 \times 2)$ reconstructions $[102,103]$.

Transient RDS signals monitored during GaAs growth were found to be highly correlated with crystal truncation rod (CTR) X-Ray intensity oscillations arising from the nucleation, growth and coalescence of islands during layer-by-layer growth [102. 103]. Similar to the RHEED case, the cyclic roughening and smoothing of the surface causes the CTR intensity to decrease and then to recover.

For technologically relevant III- $\mathrm{V}$ heterostructures RDS has been employed in both time-resolved measurements and in studies of altoy compounds and SL structures $[105,106]$. lioch et al. studied heteroepitaxy in several materials combinations: 
InGaAs, $\operatorname{In}_{0.75} \mathrm{Ga}_{0.25} \mathrm{As}_{0.5} \mathrm{P}_{0.5}, \operatorname{In}_{0.5} \mathrm{Ga}_{0.5} \mathrm{As}_{0.9} \mathrm{P}_{0.1}$ and AlInAs on $\operatorname{In} \mathrm{P}(001)$ : and In$\mathrm{GaP}$ on $\operatorname{GaAs}(001)$. When heterojunctions were grown by MOVPE. the RDS signais changed abruptly at the onset of growth of a new material. Each materials combination exhibited a characteristic "signature" which was quite reproducible and dependent upon the wavelength of illumination. The signal typically rises to a maximum and then exhibits damped oscillatory behaviour. This behaviour was attributed to interference of the light at the buried interface: the growing layer acts as a FabryPérot cavity. A model for the reflection and transmission of light at the surface and the buried interface was used to model the oscillations which occur over a time scale of several minutes, from which the layer thicknesses were determined [10:3].

Zorn $\epsilon$ al. performed RDS studies during the growth of ternary alloys on CaAs(001) with alloying on either the group III ( $\operatorname{In}_{x}\left(\mathrm{Ca}_{1-x} \mathrm{As}\right)$ or group $\mathrm{V}\left(\mathrm{GaAs_{1-s }} \mathrm{P}_{x}\right)$ sublattices [106]. Superlattice growth was also investigated for InCia.ts/CiaAs structures. in which signal oscillations with ML periodicity were used for real-time growth control. Fabry-Pérot interference effects were observed during the $\mathrm{SL}$ growths as an envelope to the RDS signals related to the growth of individual SL periods. A review article by Zorn $e t$ al. has also been published [107].

As discussed in Section 5.2. Arès $e$ al. have also used RDS to study details of InAs SQW growth by ALE [90]. Extensions of this work have been carried out and will be described in Section 7.4 . The next section will review published reports discussing PL characterization of InAs SQW structures.

\subsection{Optical Properties of Ultrathin InAs Quantum Wells in GaAs}

Apart from the interest in the InAs/GaAs system due to the fundamental materials properties and epitaxial growth challenges, there is considerable interest in the optical properties of these heterostructures. This is partly due to the fact that carriers bound to single and subML InAs depositions in GaAs represent an extreme example of quantum confinement. Additionally, such systems have exhibited sharp and intense 
PL which is highly desirable for the production of laser devices. In this section a brief summary will be given in order to introduce several mechanisms which contribute to the PL observed in these systems. This will be followed by a short review of the rather rich literature dealing with this subject.

Optical characterization of epitaxial semiconductors is essential for identifying recombination mechanisms. studying band alignment or for simple evaluation of material quality. A PL measurement involves three principal processes: excitation. thermalization and recombination [108]. A laser light source with an energy greater than the bandgap energy. $E_{g}$, of the sample is used for excitation. in which electron-hole pairs are created in the material. These may then thermalize and relax towards quasi-thermal equilibriun distributions before recombining. Radiative recombinations produce the observed PL signal. A careful examination of the spectra may then be used to identify the responsible mechanisms based on emission energies and other properties of the spectra.

In pure materials at low temperature and low excitation levels electron-hole pairs are attracted to each other by Coulomb interactions to form excitons which can move throughout the material and transport energy [108]. The energy of a free exciton. $E_{F E}$. may be expressed as

$$
E_{F E}=E_{g}-E_{X}+E_{K E} .
$$

where $E_{K E} E$ is the kinetic energy of the exciton. $E_{X}$ represents the binding energy of the exciton, where

$$
E_{X}=\frac{(13.6 \mathrm{eV})}{n^{2}} \frac{\mu}{m_{0} \varepsilon^{2}} .
$$

in terms of the dielectric constant of the material, $\_$, the electron mass, $m_{0}$. and the principal quantum number, $n$. The Bohr energy is $m_{0} e^{4} /\left(4 \pi \varepsilon_{0}\right)^{2} \cdot 2 h^{2}=13.6 \mathrm{eV}$. where $\hbar$ is Planck's constant, $\varepsilon_{0}$ is the permittivity of free space and $e$ is the electron charge. The reduced mass of the exciton is given by $\mu=m_{\mathrm{e}} m_{h} /\left(m_{\mathrm{e}}+m_{h}\right)$, where $m_{\mathrm{e}}$ and $m_{h}$ are the electron and hole effective masses. For example, the $n=1$ exciton binding energy in $\mathrm{GaAs}$ is approximately $5 \mathrm{meV}$, using the heavy-hole effective mass.

The binding energy of a free exciton can be increased by the presence of a point defect, like a neutral or ionized impurity in the material [108]. If the binding energy of 
the exciton is increased near the impurity, then the exciton can minimize its energy by becoming bound to the defect. Bound excitons show sharp PL emission because the kinetic energy term in Equation 5.25 vanishes. In GaAs. PL lines are often observed arising from excitons bound to neutral acceptor and donor impurities. These are labelled $\left(\mathrm{A}^{0} . \mathrm{X}\right)$ and $\left(\mathrm{D}^{\mathrm{U}} . \mathrm{X}\right)$ and at temperatures near the boiling point of liquid ${ }^{\mathrm{N}} \mathrm{He}$ $(4.2 \mathrm{~K})$, these transitions have emission energies of $1.512 \mathrm{eV}$ and $1.51 .5 \mathrm{eV}$. respectively. The recombination energy for a bound exciton, $E_{B E}$. is given by the expression

$$
E_{B E}=E_{y}-E_{X}-E_{L v e} .
$$

where $E_{L o c}$ is the impurity-specific localization energy.

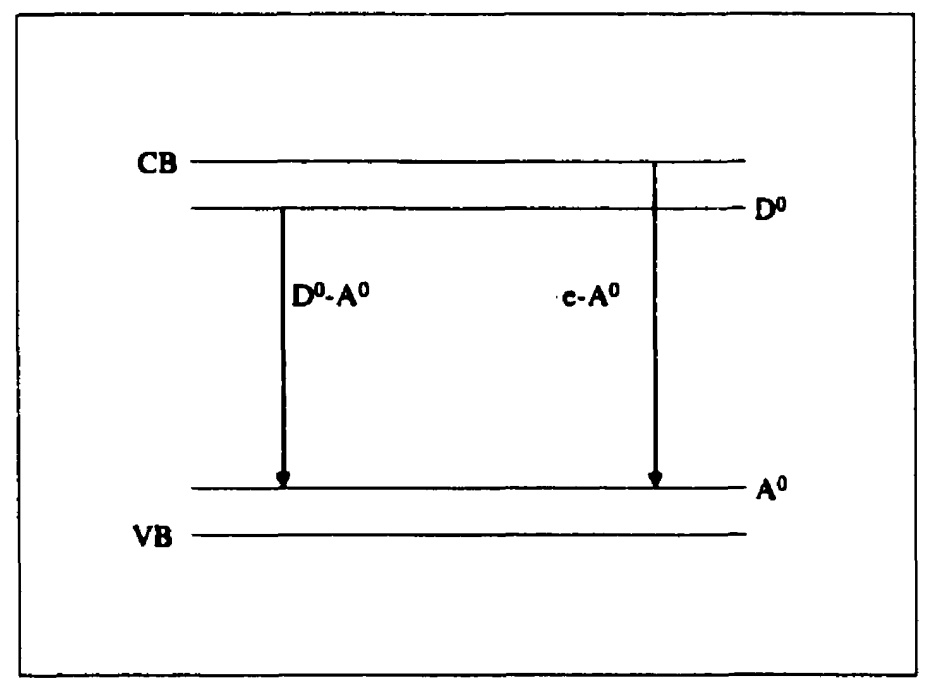

Figure 5.i: Illustration of radiative transitions involving electrons bound to neutral donors $\left(D^{0}\right)$ and holes bound to neutral acceptors $\left(A^{0}\right)$. CB and VB refer to the bottom and top of the conduction band and valence band, respectively.

Real materials contain both donor and acceptor impurities as illustrated in Figure 5.i. Under equilibrium conditions some electrons from the donors will be trapped by the acceptors. This produces ionized acceptors $\left(A^{-}\right)$and donors $\left(D^{+}\right)$in the material. Under optical excitation some of these ionized impurities may be neutralized 
by the creation of additional electrons and holes. In returning to equilibrium some of the electrons bound to neutral donors will recombine with holes bound to neutral acceptors, resulting in the emission of so-called donor-acceptor-pair (DAP) luminescence $\left(D^{0}-A^{0} \rightarrow D^{+}-A^{-}\right)$. In MOVPE GaAs, in which carbon is a shallow acceptor (occupying As crystal sites), low-T PL spectra often exhibit DAP and free-bound $\left(\mathrm{e}-\mathrm{C}^{\mathrm{U}}\right)$ transitions at 1.490 and $1.493 \mathrm{eV}$, respectively.

InAs SQWs in CiaAs are an example of isoelectronic $\delta$-doping in semiconductors [109]. InAs and Ga.As are both direct gap semiconductors with $4.2 \mathrm{~K}$ bandgaps of $0.418 \mathrm{eV}$ and $1.519 \mathrm{eV}$. respectively [53]. Heterostructures of this type have a "straddling" band lineup (type I) in which the electron and hole are both quantized in the narrow-gap material. While this quantization occurs primarily in the $\ln A$ s layer. the exciton dimensions are actually significantly larger than the InAs well-width. meaning that much of the exciton wavefunction extends into the Ga.As barrier layers. The free exciton Bohr radius is given by $a_{B}^{X}=\left(\varepsilon m_{0} / \mu\right) a_{B}^{H}$, where $a_{B}^{H}=4 \pi \varepsilon_{0} \hbar^{2} / m_{0} t^{2}$ is the Bohr radius of the Hydrogen atom $(0.529 \mathrm{~A})$. In GaAs the value is approximately $a_{B}^{X}=111 \mathrm{~A}$. In the case of an exciton confined to a quant um well the binding energy is increased. while the Bohr radius is reduced by the confinement. An interesting feature of excitons in quasi 2-dimensional systems is the splitting into light- and heavy-hole excitons resulting from the corresponding splitting of the valence-band states [109]. Under normal conditions the heavy-and light-holes are degenerate at the centre of the Brillouin zone. The lifting of this degeneracy is illustrated in the schematic of the InAs/GaAs band alignment given in Figure 5.8.

Many published reports have discussed PL emission from excitons bound to ML or subML InAs SQWs in GaAs. Experimental studies have involved samples grown using MBE [10, 11],[13]-[15],[110]-[123], MEE [12, 110], ALE [5, 124] and FME [79]. Very few articles have dealt with films grown by conventional MOVPE [i],,[125]-[12i]. To illustrate the differences in the results obtained by various researchers. Table $\mathbf{5 . 2}$ gives the PL emission energies and linewidths (where available) for InAs films with thicknesses near 1 ML. Thicker and thinner films have also been studied. Similar discrepancies (or similarities) have been observed and in general the emission energies of subML films are higher than those of 1 ML films, while the linewidths are generally 


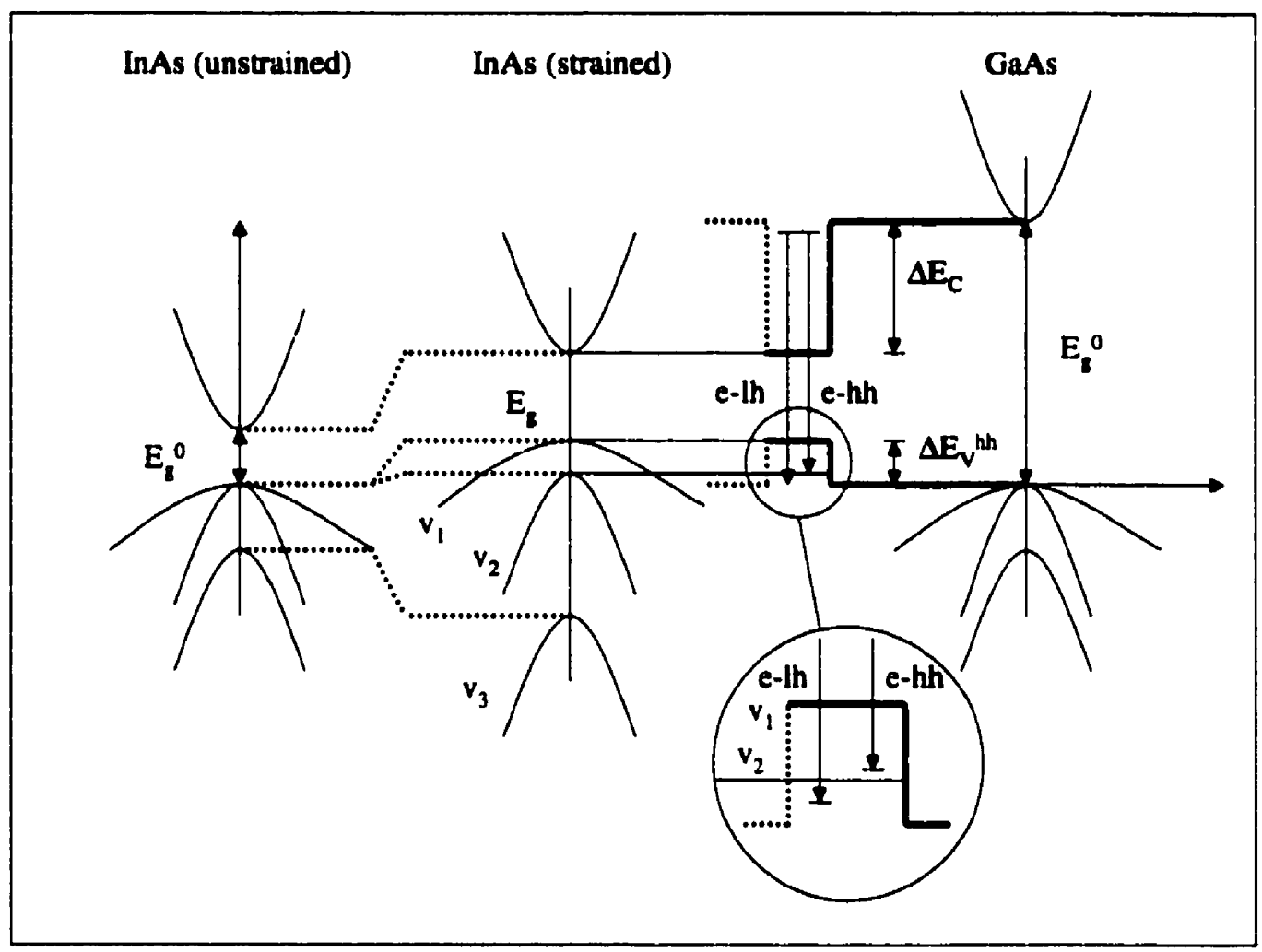

Figure 5.8: Illustration of band alignment in InAs/GaAs SQW structures (from Brandt [10]). The figure shows both the $k$-space band structure and the real-space line-up. The unstrained band structures for InAs and GaAs are given in the left and right parts. The dashed lines illustrate the shift of the $\ln A$ s band edges due to the compressive strain, leading to the situation shown in the middle. The inset shows a magnification of the heavy-hole (hh) and light-hole (lh) states and the optical transitions. indicated by arrows. 
narrower. For thicker films the converse is generally true.

SubMLs of InAs in GaAs were studied by numerous authors [5],[13]-[15], [116. $117,119,121,123]$. These studies demonstrated the sharp and intense luminescence which can be obtained by burying very small amounts of InAs within a GaAs matrix. However. no consensus has been reached on either the structural properties of the films or the nature of the optical emission. It was believed that the InAs forms island arrays which are oriented along the [01 1 ]-direction [1.5]. This islanding postulate was based on the absence of excitonic resonances in samples in which the growth was interrupted after the InAs layer deposition. which was thought to promote a uniform subML coverage. The orientation of the islands was postulated due to the anisotropy of the luminescence with polarized excitation illumination. Islanding of the InAs was believed to be a suitable mechanism for producing 0-dimensional quantum confinement [14]. although more recent work has found that the PL results may be adequately explained by 2-dimensional well-models, since In.ts islands are much smaller than the exciton radius [5, 123]. In this case the sub.ML film is treated as a conventional quantum well. with its interface roughness determined by the average InAs coverage. For subML depositions it was found that the PL emission energies increased with decreasing In.As coverage, while the linewidths generally decreased [5, 12:3]. Eventually the InAs-related luminescence merges with the GaAs bound excitonic features for very low coverages.

Light-emitting devices, e.g. laser structures, using InAs/GaAs active regions have been produced by several groups, using MBE [11, 128], ALMBE [1:2] and MOVPE [ 7 . 126]. Of these, some devices contained InAs SQWs in GaAs [i, 11, 12], while others contained $(\operatorname{InAs})_{n} /(\mathrm{GaAs})_{m}$ short-period superlattices (SPSLs) with $(n, m)=$ $(1,2)[126]$ and $(n, m)=(1,4)[128]$. It is likely that the SPSL structures actually consisted of InGaAs alloy films in the active regions due to segregation. The most recent laser results are particularly interesting due to the excitonic nature of the light emission and the high efficiency [i]. It is noteworthy that continuous wave (CW) operation of an injection-type laser employing an InAs single- or subML active region has not been demonstrated, to the best of our knowledge.

Recent theoretical developments have reemphasized the promise of ultrathin InAs 
SQWs in GaAs for highly efficient luminescence [6. 129]. Empirical tight-binding (ETB) calculations were used, and were thought to be suitable for this type of system because (i) the only input parameters are the band-structure values of the substrate and substitution bulk materials (e.g. GaAs and InAs), and (ii) ETB is an atomistic model and makes no assumption on the shape of the wave function or the interface matching conditions [129]. In the most recent calculation, lotti et al. used a Green's function tight-binding model, in which the exciton is Wannier-Mott like in its in-plane dynamics [6]. Along the growth direction the electron-hole dynamics were treated by tight-binding. thus taking the band-folding along the growth direction into account. The band-folding results in a larger effective mass for both types of carriers. Measuring energies from the top of the CiaAs valence-band. this calculation predicted heavy-hole and CB levels bound at 233 and $1468 \mathrm{meV}$ for 1 ML InAs insertions. The corresponding localization energy of the ML CB measured from the bottom of the GaAs CB was predicted to be $52 \mathrm{meV}$. The study also suggested that the exciton has a binding energy of $12.9 \mathrm{meV}$ and so excitonic effects should be observable at room temperature. Additionally, the oscillator strength per unit area was predicted to be $12.9 \times 10^{12} \mathrm{~cm}^{-2}$ which is between 3 and 5 times as large as the oscillator strength in an $\operatorname{In}_{r} \mathrm{Ga}_{1-x} \mathrm{As} / \mathrm{GaAs} \mathrm{QW} 80 \mathrm{~A}$ wide [6]. These enhancements arise because of the increased wave-function localization, large in-plane masses and in-plane nonparabolicity, all of which tend to increase the binding energy and the oscillator strength.

In this chapter it has been shown that the InAs/GaAs system has been the subject of considerable research recently. Various issues have been discussed. including the structure and epitaxial growth of ultrathin films with thicknesses below the critical thickness, beyond which the growth becomes three-dimensional in nature. In fact. growth in the (3-D) Stranski-lirastanov regime and films produced by this mechanism are also the subject of extremely intense study at this time. In this chapter growth methods for avoiding In segregation have been discussed, and will allow an interpretation of the experimental data presented in Chapter $i$. Similarly, optical characterization using in-situ RDS and ex-situ PL measurements have been discussed in order to create a context for the related work which has been performed in this thesis. Chapter 6 will now describe the experimental methods employed in this work. 


\begin{tabular}{|c|c|c|c|c|c|}
\hline Ref. & Description & $t \quad t[\mathrm{ML}]$ & $T[\mathrm{~K}]$ & $\overline{E_{P L}[\mathrm{eV}]}$ & $\overline{\Gamma_{P L}[\mathrm{meV}]}$ \\
\hline [5] & ALE SQWs. flashoff & 1 & 1.8 & 1.460 & $i$ \\
\hline$[\bar{\imath}]$ & MOVPE laser structure & 1.5 & 2 & 1.12 & 10 \\
\hline [10] & MBE SQW. Hashoff & 1.2 & 4.2 & 1.417 & 22 \\
\hline [11] & MBE laser structure, $T_{g}=5.50^{\circ} \mathrm{C}$ & $\overline{1}$ & $i i$ & 1.471 & - \\
\hline$[13]$ & MBE SQW, flashoff & 1 & 8 & 1.441 & 8.0 \\
\hline [14] & MBE subMLs, flashoff & $0.8^{3}$ & 6 & 1.465 & - \\
\hline [1.5] & MBE SQWs. flashoff & $\mathrm{T}$ & 5 & 1.463 & - \\
\hline [79] & FME SQW. $T_{g}=500^{\circ} \mathrm{C}, \mathrm{AsH}_{3}$ & T & 2 & 1.483 & 3.2 \\
\hline$[8-1]$ & ALMBE SQW,$T_{g}=360^{\circ} \mathrm{C}$ & 1 & 10 & 1.454 & - \\
\hline$[8+1]$ & ALMBE SQW,$T_{g}=460^{\circ} \mathrm{C}$ & 0.9 & 10 & 1.479 & - \\
\hline$[8-1]$ & MBE SQW. $T_{g}=420^{\circ} \mathrm{C}$ & $T$ & 10 & 1.444 & - \\
\hline$[8+1]$ & MBE SQW,$T_{g}=480^{\circ} \mathrm{C}$ & I & 10 & 1.459 & - \\
\hline [110] & MEE SQW. $T_{g}=350^{\circ} \mathrm{C}$ & 0.9 & 10 & 1.48 & 6 \\
\hline$[110]$ & MBE MQW $T_{g}=550^{\circ} \mathrm{C}$ & 1.2 & 10 & 1.45 & 8 \\
\hline [111] & MBE MQW. flashoff & 0.8 & 4.2 & 1.47 & 5 \\
\hline$[112]$ & MBE MQW. flashoff & 0.8 & 4.2 & 1.47 & - \\
\hline$[113]$ & MBE SQWs. $T_{g}=420^{\circ} \mathrm{C}$ & $\mathrm{T}$ & - & 1.461 & - \\
\hline$[114.115$ & MBE SQWs, $T_{y}=380^{\circ} \mathrm{C}$ & 0.92 & 6 & 1.442 & 7.5 \\
\hline [114] & MBE SQWs, $T_{g}=430^{\circ} \mathrm{C}$ & 0.92 & 6 & 1.452 & 5 \\
\hline [114] & MBE SQWs, $T_{g}=480^{\circ} \mathrm{C}$ & $\overline{0.92}$ & 6 & $1.4 i$ & 2.5 \\
\hline [121] & MBE. $T_{g}=400^{\circ} \mathrm{C}$, cap at $580^{\circ} \mathrm{C}$ & 1 & 11 & 1.43 & - \\
\hline [122] & MBE SL, 10 periods, $T_{g}=500^{\circ} \mathrm{C}$ & 0.85 & 10 & 1.478 & 6 \\
\hline [123] & MBE SQWs, flashoff & l & 10 & 1.4635 & 9.5 \\
\hline$[125]$ & MOVPE SQWs. $T_{g}=730^{\circ} \mathrm{C}, \mathrm{AsH}_{3}$ & 1.03 & 4 & 1.491 & 5 \\
\hline$[12 i]$ & MOVPE SQW & $\bar{I}$ & 5 & 1.472 & 6 \\
\hline
\end{tabular}

Table 5.2: Experimental PL energies, $E_{P L}$, and full-widths at half maxima. $\Gamma_{P L}$, reported for InAs/GaAs heterostructures, measured at temperature, $T$. The thicknesses of the InAs layers, $t$, are given in units of MLs, as quoted by the authors. The definition of the thickness of a strained InAs layer in $\mathrm{GaAs}(001)$ is not consistent among all authors. 


\section{Chapter 6}

\section{Experimental Details}

In this chapter the details of the MOVPE technique will be presented. This will be followed by a discussion of the in-situ and ex-silu characterization which was carried out at SFU. Sections 6.6 and 6.7 will then describe the setups used at the various synchrotron beamlines used in these experiments.

\subsection{The MOVPE System}

The MOVPE system used in this research was manufactured by Thomas Swan and includes a commercial gas handling unit and computer-control software [16]. An illustration of the system appears in Figure 6.1. Ultrahigh purity (UHP) hydrogen is used as the carrier gas, while liquid nitrogen $\left(\mathrm{LN}_{2}\right)$ is evaporated to provide the vent-line gas supply. Reactor gases travel through high purity stainless steel tubing, making use of $\mathrm{VCR}^{T M}$ connectors to provide metal, as opposed to elastomer. seals.

As mentioned in Chapter 2. the stainless steel bubblers containing the metalorganic sources are maintained at constant temperature using ethylene glycol baths. The bubbler pressures are controlled electronically to provide stable. reproducible conditions. During a growth sequence $\mathrm{H}_{2}$ gas is passed continuously through the relevant bubblers to achieve steady-state conditions. The run-to-vent switching system enables the saturated group III or group V vapours to be directed to carrier or vent lines as required by the growth recipe. Separate alkyl and hydride carrier lines are 


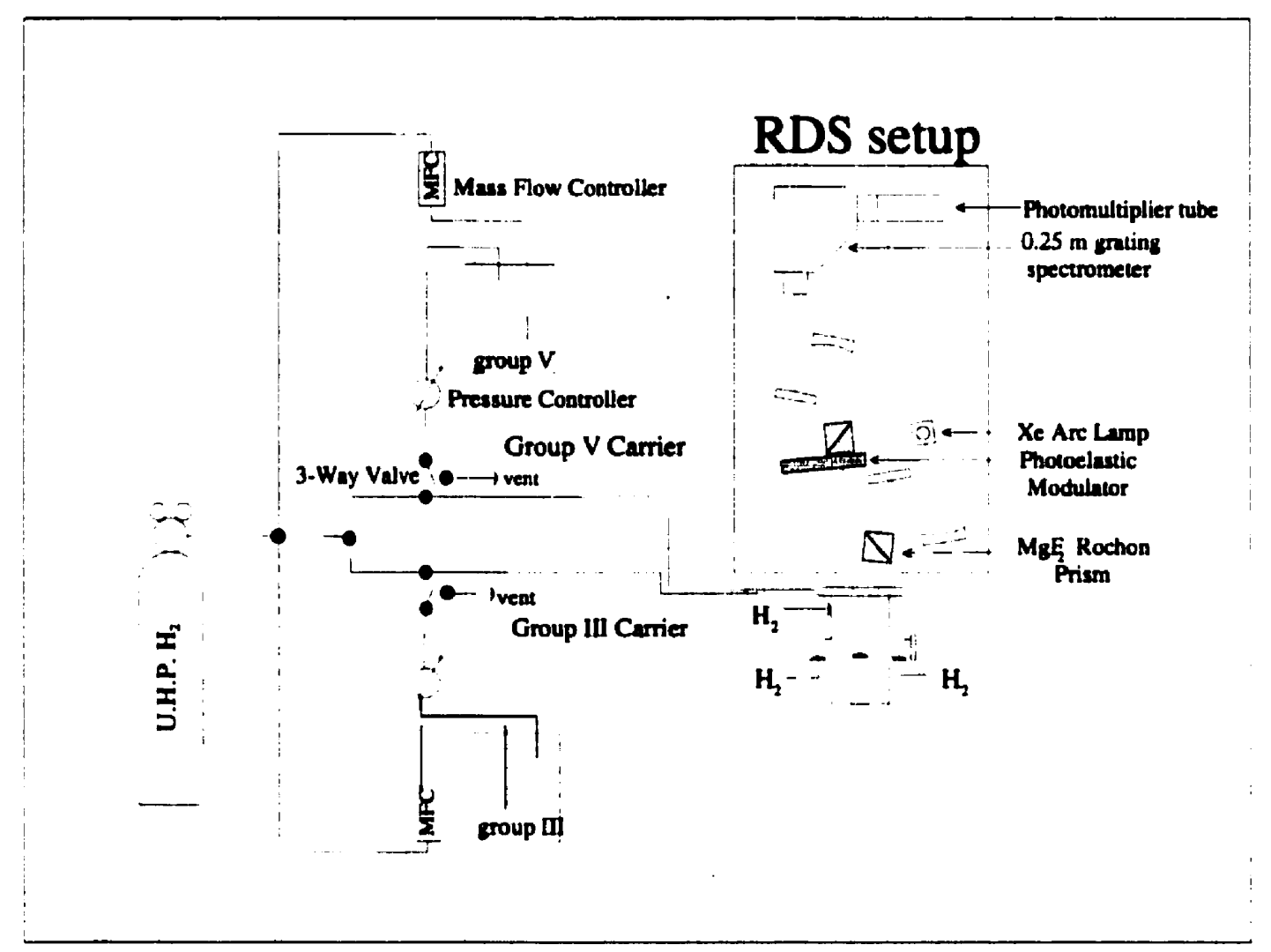

Figure 6.1: A schematic of the vertical MOVPE reactor and associated gas flow lines. The figure includes the RDS system which allows in-situ characterization of the growing films.

used to prevent intermixing between the saturated vapours.

Several techniques are employed by the system to ensure steady, laminar reactor flows. The total gas flow passing through both carrier lines is kept constant by a computer-controlled makeup flow. During run-to-vent gas switching, the makeup line compensates for the reduced amount of gas to preserve the total flow of the line and hence to avoid turbulent flows. Additionally, the run and vent lines are kept at equal pressures using a differential pressure controller which makes small adjustments to the vent flow as required. 
The vertical growth reactor employed in this work features stainless steel construction with continuous water cooling. The design is similar to that described by Brennan $t$ al. with optical ports for normal incidence and Brewster angle measurements [104]. Carrier lines enter the top of the chamber while purge lines are used to maintain laminar flow in the reactor and to keep the optical windows clean. The carrier gases mix in the top of the reactor and travel downwards through a quartz nozzle which directs the saturated vapours in a laminar flow pattern towards the susceptor and aids in reducing the quantity of wasted material. The volume surrounding the cylindrical nozzle is purged with hydrogen gas to assist in maintaining laminar flow. A summary of the growth conditions appears in Table 6.1. The next section describes the RDS system shown in Figure 6.1.

\begin{tabular}{|c|c|c|}
\hline Parameter & ALE & MOVPE \\
\hline Reactor pressure & 50 Torr & 50 Torr \\
\hline Alkyl total flow & $500 \mathrm{sccm}$ & $2.50 \mathrm{sccm}$ \\
\hline Hydride total flow & $500 \mathrm{sccm}$ & $250 \mathrm{sccm}$ \\
\hline Total gas flow & $4.6 \mathrm{slm}$ & $2.3 \mathrm{sIm}$ \\
\hline Window purge flow & $20 \mathrm{sccm}$ & $20 \mathrm{sccm}$ \\
\hline Gas velocity & $50 \mathrm{cms}^{-1}$ & $25 \mathrm{cms}^{-1}$ \\
\hline \hline
\end{tabular}

Table 6.1: Summary of ALE and MOVPE growth conditions.

\subsection{RDS Measurements}

The RDS apparatus is mounted on a vertical optical table above the reactor chamber to allow normal incidence measurements. An extensive discussion of the RDS technique has been given by Arès [16]. Light is produced by a Xe arc lamp and directed onto the sample's surface using front surface focusing optics. The path from the lamp to the sample contains a $\mathrm{MgF}_{2}$ Rochon prism which polarizes the light. The linearly polarized light is then incident upon the sample at $45^{\circ}$ to the principal crystal axes (the crystal directions (110) and (110)). The electric field components along these two directions are initially equal. The reflected light has different components along the two directions because of anisotropy on the sample surface. 
Figure 6.1 shows the photoelastic modulator unit which is part of the system for analyzing the reflected light. The photoelastic modulator contains a quartz bar which becomes birefringent under stress induced by a piezoelectric cell. This stress is modulated at $50 \mathrm{kHz}$ using an electronic controller to produce retardations between $-\lambda / 2$ and $\lambda / 2$. The main axis of the photoelastic modulator is positioned to coincide with the polarization direction of the light prior to reflection. The components of the polarization of the reflected light are analyzed using a quartz Rochon prism. Finally a $0.25 \mathrm{~m}$ grating spectrometer is used to monitor the signal with a photomultiplier tube having a wide spectral response multialkali photcathode.

The intensity of the light reaching the detector contains a bulk. isotropic signal and a small modulation due to the anisotropic surface. A lock-in amplifier is used to measure the amplitude of the modulated signal. which is about $10^{-1}-10^{-5}$ times that of the DC component. An electronic control circuit is used to maintain a constant $D C$ signal level by varying the gain (and hence the sensitivity) of the photomultipler tube.

A full RDS spectrum (1.j-j el') cannot be taken sufficiently quickly to permit time-resolved measurements. During a standard growth sequence on Cia.As. for example, it is often preferable to monitor the RDS signal at a fixed energy. As mentioned in Section 5.3, a suitable energy is $2.6 \mathrm{el}$. which exhibits strong features related to transitions within As dimers along the surface [99]. RDS transient signals obtained in this way allow the observation of surface anisotropy developments with very high temporal resolution $(\mathrm{SHz})$. This method is particularly useful for observing the crystal surface during ALE growth.

RDS spectra contain contributions not only from the surface, but from artifacts related to stress in the optical window, etc. It is possible to correct for these effects by recording a spectrum in which the sample is rotated $90^{\circ}$ from its original orientation. This can be done at the beginning of a growth experiment and then used to correct the spectra obtained during the experiment. This is possible because

$$
\left(\frac{\Delta r}{r}\right)_{o p t}=\left(\frac{\frac{\Delta r}{r}\left(0^{\circ}\right)+\frac{\Delta r}{r}\left(90^{\circ}\right)}{2}\right)=\left(\frac{\frac{\Delta r}{r_{s a m}}+\frac{\Delta r}{r_{o p t}}-\frac{\Delta r}{r_{s a m}}+\frac{\Delta r}{r} \text { opt }}{2}\right)
$$

where $(\Delta r / r)_{s a m}$ and $(\Delta r / r)_{\text {opt }}$ are the contributions to the real part of the RDS 


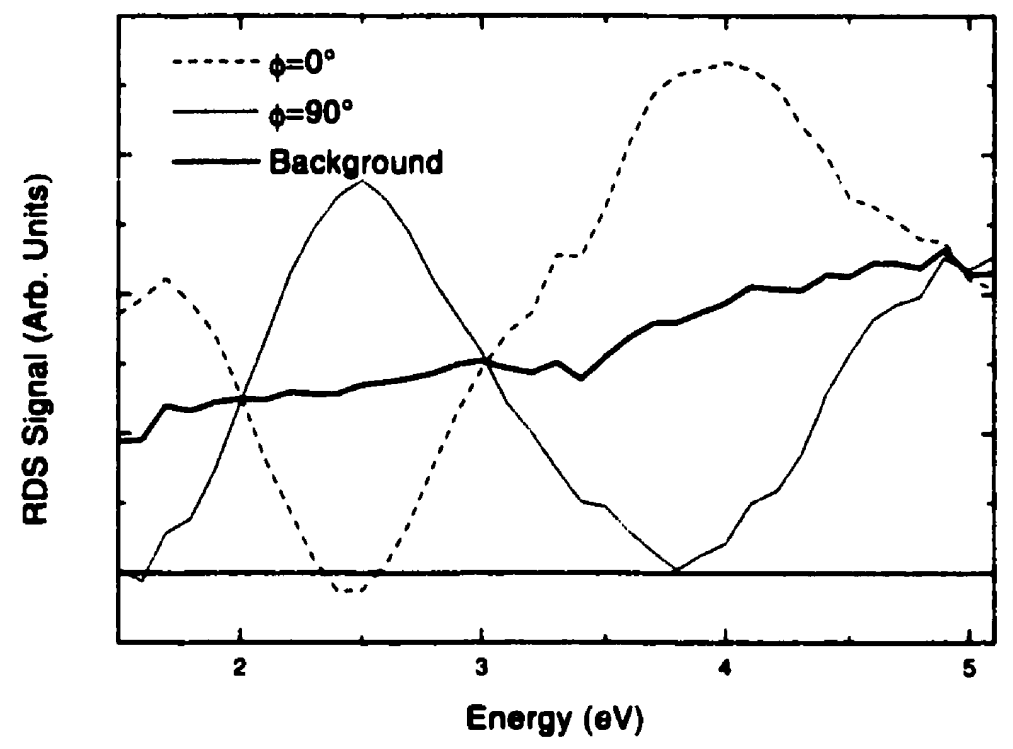

Figure 6.2: RDS data obtained in two orientations. 0 . used for determining the background signal. The background may be removed from the data to obtain correct relative levels.

signal due to the sample and the optics. respectively. An example of this process is shown in Figure 6.2.

In order to evaluate the development of surface anisotropy during the MOVPE growth of InAs QWs in GaAs, the real-time spectroscopic method of Aspnes was employed [101]. As described in Section 5.3. this exploits the reproducibility of a given growth sequence to obtain time-resolved data throughout the spectral range of interest. A single transient signal at fixed energy was recorded for each cycle of a GaAs/InAs SL growth on $\operatorname{GaAs}(001)$. The data were then reorganized to provide details of the surface anisotropy throughout a single growth cycle. The results will be presented in Section 7.6.1. The next section describes the atomic force microscopy measurements which form an important part of the ex-situ sample characterization performed at SFU. 


\subsection{Atomic Force Microscopy}

After preparing an epitaxial sample it is useful to study the physical morphology of the sample surface. This can be done on different length scales using the naked eye, optical. atomic force or scanning tunnelling microscopes. This section will describe the AFM technique which is suitable for studying arcas on the sample surface from $100 \times 100 \mu \mathrm{m}^{2}$ to $1 \times 1 \mu \mathrm{m}^{2}$ with relative ease. Higher-resolution scans, even at the atomic scale. are also possible but are more difficult.

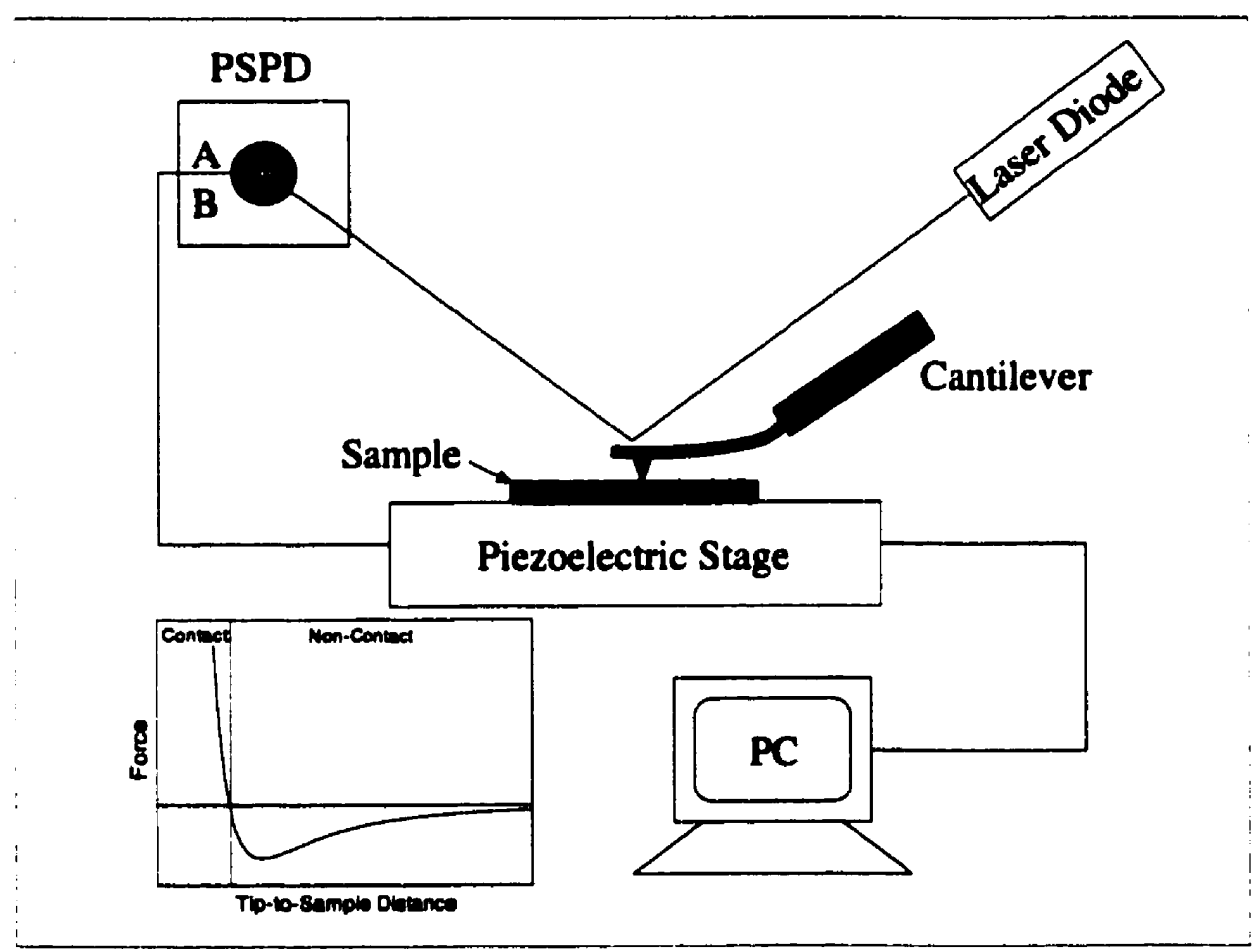

Figure 6.3: A schematic of the atomic force microscope. ' $A$ ' and ' $B$ ' refer to the two PSPD elements. The inset shows the interaction between the AFM tip and the sample surface. The contact mode was used to obtain the images presented in this thesis.

AFM uses a cantilever upon which is mounted a sharp tip which is placed near the sample surface (Figure 6.3). Interactions between the tip and the surface produce 
measurable changes which are used to study the surface. These interactions are illustrated in Figure 6.3. In this work, measurements were made in the "contact" mode. Here the tip comes in intimate contact with the sample surface (a few angstroms) and experiences a repulsive force. $A$ visible laser beam deflects off the cantilever towards a position-sensitive photodetector (PSPD). Using a piezoelectric stage. the sample is rastered beneath the tip while the deflections are measured using the PSPD and an electronic feedback mechanism. In the measurements described here, the system was operated so that a constant force was applied to the tip. Changes in the surface topography produce changes in the PSPD signal which result in the adjustment of the height of the piezoelectric stage. The size of the necessary adjustment provides information on the height of features in the surface. Atomic scale imaging requires the constant height mode. where the spatial variation of the cantilever deflection is used directly to generate the topographic image.

\subsection{PL Spectroscopy}

The PL measurements included in this thesis were performed by Dale Harrison. working in Dr. Nichael Thewalt s laboratory. A more detailed discussion of the technique may be found in Dr. Harrison s Ph.D. thesis [108].

Briefly, a PL system consists of four main components: a light source, a cryogenic dewar. a spectrometer and a detector. In this work the samples were mounted on a holder which was then immersed in the "tail" of a Janis immersion cryostat. The dewar system was precooled using $\mathrm{LN}_{2}$ approximately 24 hours before the measurements were taken. Liquid helium $\left(\mathrm{L}^{4} \mathrm{He}\right)$ was later introduced into the chamber to allow low temperature measurements $(4.2 \mathrm{~K}$ ). Measurements of the vapour pressure were obtained using a calibrated pressure gauge which allowed a determination of the $\mathrm{L}^{4} \mathrm{He}$ bath ternperature.

The tail of the dewar contains four quartz windows for sample excitation and signal acquisition. as illustrated in Figure 6.4. In this work the samples were illuminated by an $\mathrm{Ar}^{+}$-ion laser in $\mathrm{CW}$ operation. PL signals were measured using a Bomem DA8 Fourier transform spectrometer. This is essentially a Michelson interferometer. 


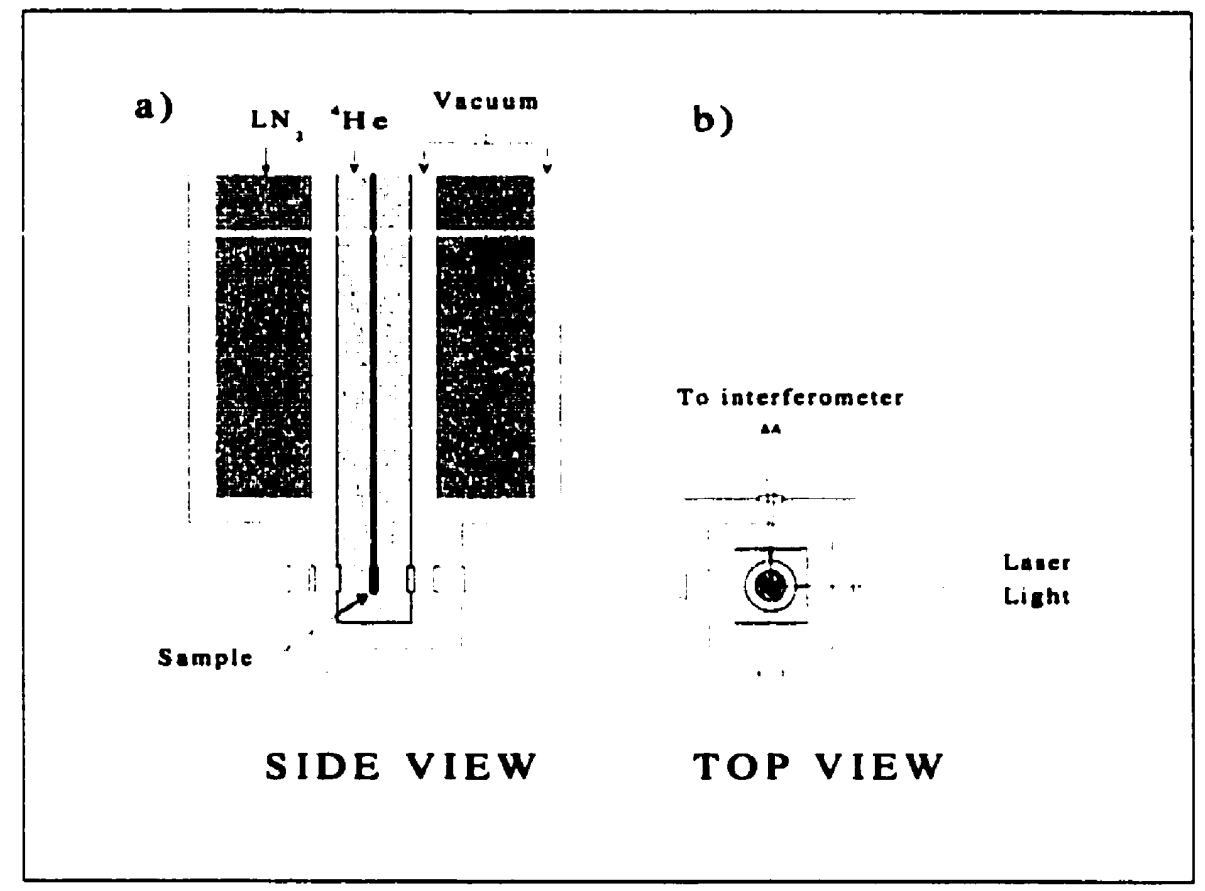

Figure 6.4: a) Liquid He cryostat used in PL measurements. b) The geometry for sample illumination and signal acquisition. From Reference [108].

Interferograms were obtained as a function of path difference between the two branches of the interferometer. The data were then converted to intensity versus energy spectra using the Fast-Fourier Transform (FFT) algorithm. In the data presented in this thesis the intensity of the PL signals were recorded using a Si avalanche photodetector [108].

In the following sections the X-Ray techniques used in this work will be discussed. The first section will describe the method used in XRD measurements using a $\mathrm{Cu}$ anode source at SFU. This will be followed by details of the synchrotron measurements performed elsewehere. 


\subsection{High-Resolution XRD}

In the SFU laboratory XRD measurements are routinely used to determine growth rates. sample composition and layer quality. A BEDE $D^{3}$ (direct-drive diffractometer) system is used for the measurements in conjunction with a Philips generator and BEDE suftware for computer cuntrul. ('u-K X-Rays (the $\mathrm{Cu} K_{\alpha_{1}}$ wavelength is $1.54056 \mathrm{~A}(8047.78 \mathrm{eV}))$ are produced by a Philips long-fine-focus vacuum tube (usually operated at $40 \mathrm{kV}$ and $30 \mathrm{~mA}$ ) and are detected using a BEDE enhanced dynamic range (EDR) detector after diffraction. A schematic of the system is shown in Figure 6.5.

The BEDE system allows the incident $X$-Rays to be collimated in two modes, allowing high intensity. low-resolution or lower intensity. high-resolution measurements. This is accomplished using a pair of channel-cut crystals. each of which contains two separate channels for asymmetric or symmetric reflections. These crystals also act as a monochromator $(\mathrm{MC})$, choosing ( $u-K_{n 1}$ radiation. In the high intensity mode, two asymmetrically cut $\mathrm{Si}(220)$ crystals are used with a total of four reflections (angular divergence $\sim 12$ arcsec). The high-resolution mode uses eight reflections from two symmetric $\mathrm{Si}(220)$ crystals (angular divergence $\sim 5$ arcsec). After Bragg collimation the X-Ray beam is shaped by a fixed entrance slit which defines the beam in the $x$ and $y$ directions.

In the alignment of rocking curve measurements. the coarse motions of the sample and detector are adjusted by $\theta$ and $2 \theta$ stages, which control movements of the order of degrees. Fine adjustments and the actual measurements are performed using precision motors for movements of the order of arcseconds. These are sometimes referred to as $\omega$ and $2 \omega$ motions. To align the sample for the measurement of what are typically very narrow rocking curves, it is necessary to adjust the sample position by rotating about an axis parallel to the sample diffracting planes and the (horizontal) plane of incidence. The alignment is achieved through an iterative optimization process employing movements about this axis and $\omega$-motions. Meanwhile. the rocking curve width and intensity are monitored by the detector. For GaAs crystals the symmetric (004)-reflection is typically observed to have a full-width at half maximum (FWHM) 


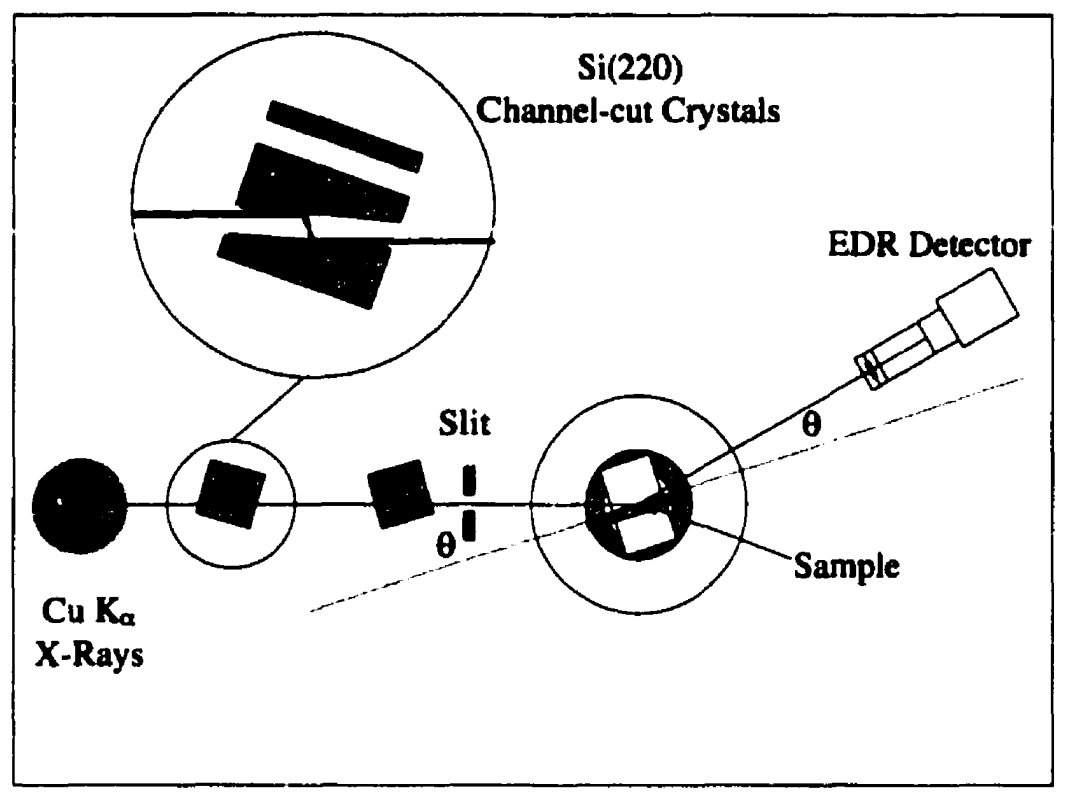

Figure 6.5: The BEDE $\mathrm{D}^{3}$ system. The inset shows the high-intensity configuration in which the beam is collimated by two asymmetric $\mathrm{Si}(220)$ reflections in each channelcut crystal.

of 8 arcsec and 16 arcsec for the high and low-resolution modes, respectively.

Rocking curves obtained in this way are compared with dynamical diffraction simulations using the BEDE RADS software. The software is quite versatile and is suitable for use with group IV and III-V zincblende semiconductors. including ternary and quaternary compounds. Simulations are prepared by defining the composition and thickness of the epitaxial layers beginning with the substrate and working upwards. The substrate thickness is assumed infinite as discussed in Section 3.3. RADS can be used with arbitrary reflections and with either $\sigma$ or $\pi$ polarizations or a combination of the two, which is most appropriate with a $\mathrm{Cu}$-anode. To include the effects of the channel-cut crystals it is possible to include a correlation function. The rocking curve for the sample is initially calculated over a range double that specified by the user. This prevents convolution artifacts at the edges of the desired range. The rocking 
curve correlation function due to the channel-cut crystals is then calculated and convoluted with the sample curve to produce a realistic profile for the true arrangement over the specified range. It is important to note that the simulations are usually performed using a logarithmic scale for the reflectivity. Many of the interesting features in the diffraction patterns are only apparent when the data are presented in this manner.

In the following section the XSW measurement technique is described. While the same physics is responsible for the dynanical diffraction occurring in the case of X-Ray's from a Cu-anode or from a synchrotron source, there are many features unique to the latter. This is particularly true in the case of the back-reflection Bragg geometry employed here.

\subsection{X-Ray Standing Wave Measurements}

XSW measurements were performed at NIST beamline .244 at the NSLS. This section provides a description of the apparatus and the techniques used in these measurements.

Synchrotron radiation results from the bending of electron (or positron) trajectories as the relativistic particles travel in closed circular orbits [1:30]. In practice a "bunch" of electrons is inserted into a UHV storage ring where they are accelerated to velocities approaching the speed of light. Around the ring, magnets are used to bend the electron paths, resulting in the emission of electromagnetic radiation. During the course of these experiments the NSLS storage ring was operating at $2.6 \mathrm{Gel}$. Shortly after the injection of electron bunches into the ring, the electron current was typically about $300 \mathrm{~mA}$. During the twelve hours before the next injection. this current decayed slowly to approximately $150 \mathrm{~mA}$. The decay rate was sufficiently slow to be negligible during the course of a single spectrum acquisition $(\sim 15$ minutes $)$. The photons are actually emitted in pulses of nanosecond duration, but for the purposes of most experiments the radiation is essentially continuous.

The radiation produced at a synchrotron source encompasses a broad spectral range, from microwaves to hard X-Rays and $\gamma$-Rays. The natural collimation of the 
emitted radiation has the advantageous property that it is confined to a narrow range of vertical angles, centred at the plane of the reference electron orbit. In the plane of the orbit the radiation is linearly polarized, while it is elliptically polarized elsewhere. One of the most attractive features is the high photon flux, which is essential for measurements of extremely dilute samples. such as the In MLs and subMLs considered here.

In beamline $\times 24 \mathrm{~A}$. "white" light from the ring enters the beamline and travels downstream towards the experiment with no vacuum interruptions. For the energies employed in this study $(\sim 4386 \mathrm{eV})$, the typical beryllium windows were not suitable and were not present anywhere in the beampath. At these energies the attenuation due to the windows would be excessive, thus the entire beamline operates under high vacuum of approximately $10^{-8}-10^{-9}$ Torr.

The white $\mathrm{X}$-rays were collimated by a Ni-coated spherical $\mathrm{Al}$ mirror located $6.8 \mathrm{~m}$ from the source. and then incident on the monochromator. $0.9 \mathrm{~m}$ further downstream. These experiments used a pair of $\mathrm{Si}(20)$.MC crystals as shown in Figure 6.6. With this configuration the energy resolution was approximately $\triangle E / E=5 \times 10^{-5}$ at $\sim 4386 \mathrm{eV}$. The motion of the crystals was controlled by stepper motors interfaced to a Digital Equipment Corporation PDP11/i3 microcomputer.

Approximately $4 \mathrm{~m}$ upstream from the experiment the monochromatic $X$-rays were collimated in the $x$ and $y$ directions by a pair of adjustable slits. Finally a fixed entrance slit $\sim 1 \mathrm{~m}$ upstream from the experimental chamber cut the beam down to approximately $1.5 \mathrm{~mm}$ (vertical) $\times 10 \mathrm{~mm}$ (horizontal).

The semiconductor samples were mounted within a stainless steel vacuum chamber as shown in Figure 6.i. The chamber was evacuated by a combination of rotary, turbomolecular and ion pumps (not depicted in the figure), with the pressure monitored by an ion gauge and a quadropole mass spectrometer. The vacuum components were interlocked so that a local vacuum failure would not vent the entire storage ring to atmosphere.

Sample alignment was accomplished using a manipulator mounted on a linear bellows and attached to the aluminum base upon which the samples were mounted. The manipulator rod was constructed from Macor to allow the aluminum base to be 


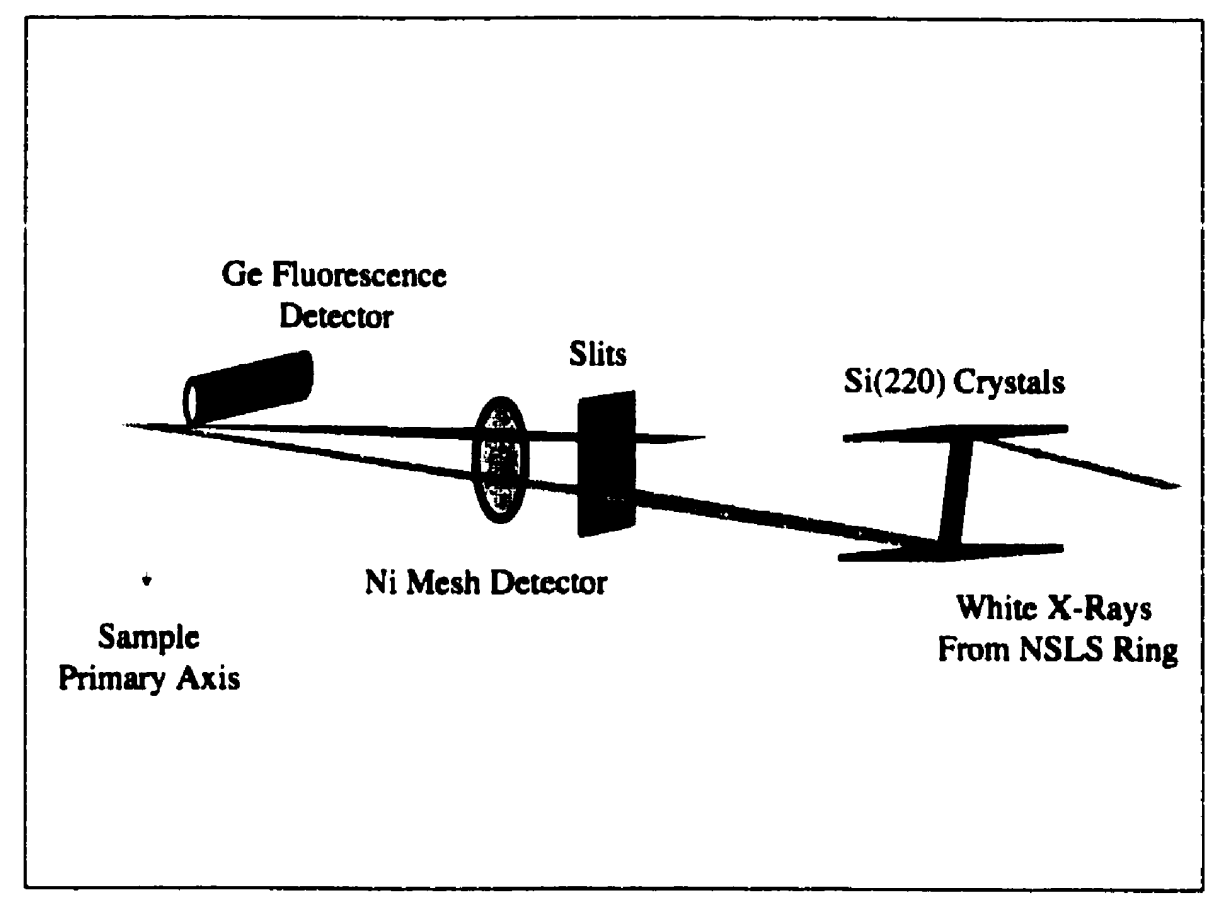

Figure 6.6: A schematic of the experimental back-reflection XSW arrangement at beamline X:4A at the NSLS (not to scale).

electrically isolated from the chamber. No azimuthal adjustments were possible with this apparatus.

As shown in Figures 6.6 and 6.T, the relative incident and reflected beam intensities were measured by monitoring the current. $I_{0}$, through a fine $\mathrm{Ni}$ mesh between the entrance slit and the sample. The current signal was passed by coaxial cable to a preamplifier placed very close to the mesh, and then to a voltage/frequency converter.

The true sample configuration differed slightly from the ideal back-reflection geometry. The entrance/exit slit depicted in Figure 6. 7 was coated in phosphor to permit visual observation of the incident and reflected beams; the phosphor glowed bright green under X-ray illumination. At the $\mathrm{GaAs}(004)$ back-reflection, the incident beam was observed to pass through the entrance slit, while the back-reflected beam passed 


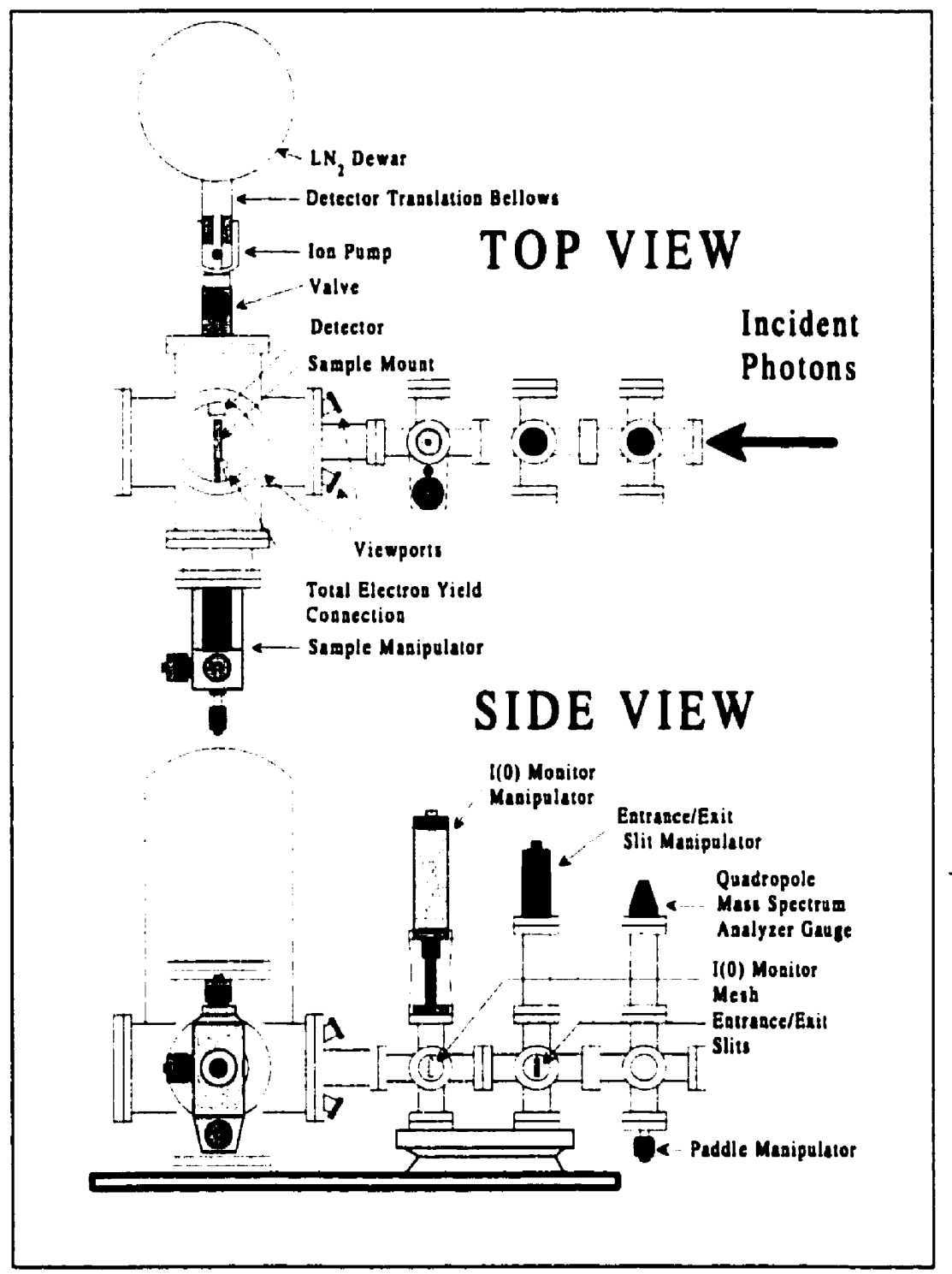

Figure 6.7: A schematic of the experimental chamber used in XSW measurements at the NSLS. 
through a second slit $5 \mathrm{~mm}$ above. With a slit-sample distance of approximately $1 \mathrm{~m}$. this corresponds to a (negligible) deviation from the ideal geometry of about $0.3^{\circ}$.

As illustrated in Figure 6. $i$, the XSW signal from the substrate was monitored through measurements of the total electron yield (TEY). A wire connected to the aluminum sample mount was passed to a nearby preamplifier. with the resulting current signal carried to a voltage/frequency converter.

The fluorescence detector employed in these measurements was a CANBERRA Model GUL0110P Ultra Low Energy Germanium PIN diode with an active area of $100 \mathrm{~mm}^{2}$. As shown in Figure 6.7. the detector was introduced into the experimental chamber using a linear translation stage with bellows. Detector cooling was achieved with a $\mathrm{LN}_{2}$ cryostat/cold finger assembly to reduce thermal noise.

A schematic of the detector and associated electronics is shown in Figure 6.8. Under reverse bias. the $\mathrm{P}^{+}(\mathrm{B})-\mathrm{I}_{-} \mathrm{N}^{+}(\mathrm{Li})$ Ge homojunction is sensitive to ionizing radiation incident in the intrinsic region. The atomic symbols in parentheses denote the dopant in each Ge layer. The energy of each deposited photon results in the production of a proportional number of charge carriers, which are swept by the bias voltage into the $\mathrm{P}$ and $\mathrm{N}$ electrodes. This signal is then converted to a voltage pulse by the integral charge-sensitive preamplifier.

\begin{tabular}{|c|c|c|c|}
\hline \hline Element & $\mathrm{L}_{\alpha 1}[\mathrm{eV}]$ & $\mathrm{L}_{\alpha 2}[\mathrm{eV}]$ & $\mathrm{L}_{\beta^{\prime}}[\mathrm{eV}]$ \\
\hline $\mathrm{Ca}$ & 1097.92 & 1097.92 & 1124.8 \\
\hline $\mathrm{As}$ & 1282.0 & 1282.0 & 1317.0 \\
\hline $\mathrm{In}$ & 3286.94 & 3279.29 & 3487.21 \\
\hline \hline
\end{tabular}

Table 6.2: X-Ray emission energies (from Bearden [131]).

Figure 6.8 shows the Be and the polymer/Aluminum windows mounted to the detector. The former was mounted specifically for these experiments to reduce the fluorescence signal due to $\mathrm{Ga}$ and $\mathrm{As}$ in the samples. Fluorescence energies for the elements of interest and within our detection range are given in Table 6.2. The attenuation of $\mathrm{X}$-rays in matter can be expressed as

$$
I_{t}=I_{0} e^{-\mu t},
$$




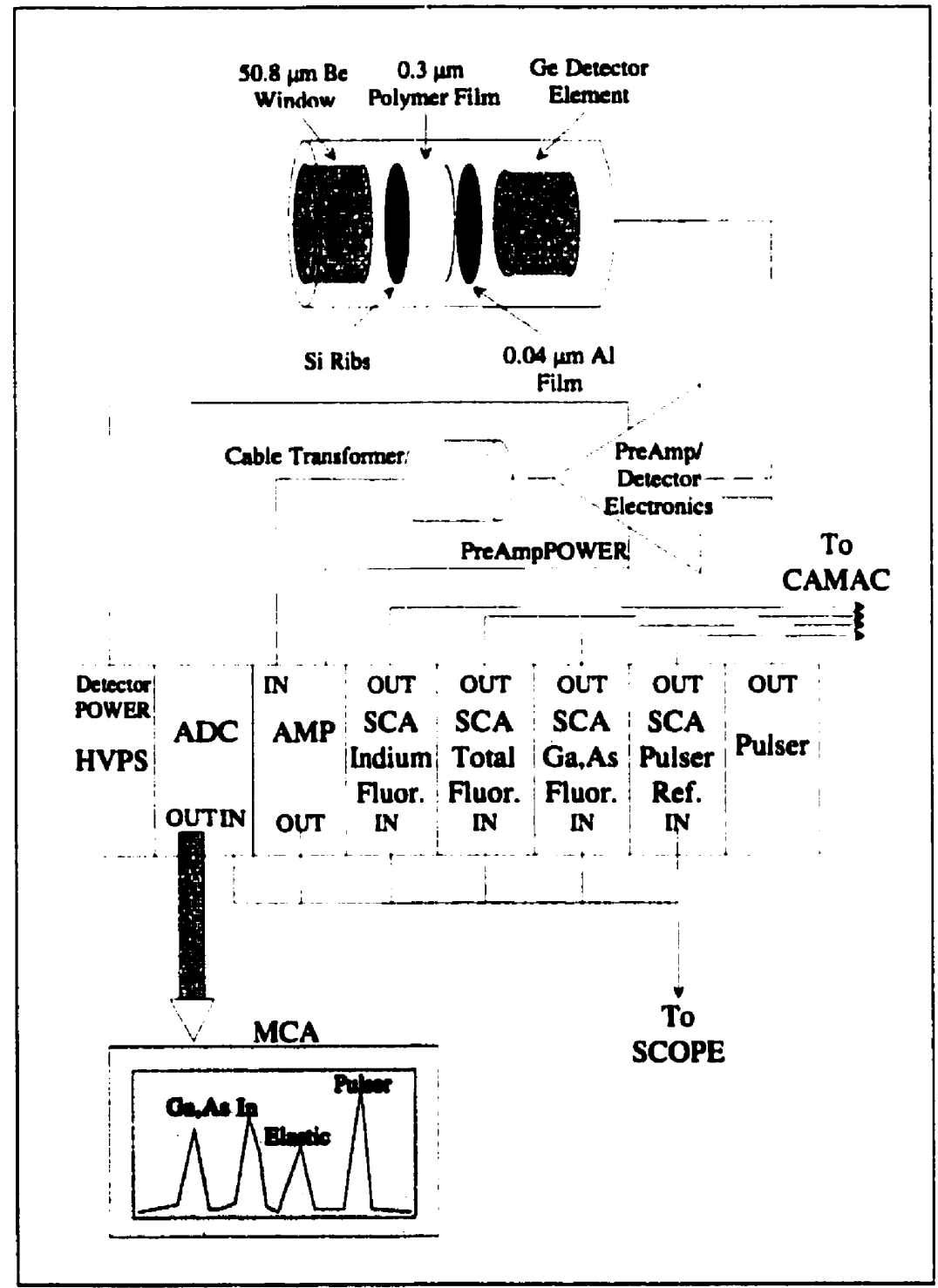

Figure 6.8: The Ge fluorescence detector and NIM electronics used in X-ray standing wave measurements. 
where $I_{0}$ and $I_{t}$ are the incident X-Ray intensity and the intensity at a depth, $t$, in the material. Here $\mu$ is the linear absorption coefficent. Fron Figure 6.9 it is apparent that a window of thickness $50.8 \mu \mathrm{m}$ ( 2 mil) causes significant attenuation of the $\mathrm{Ga}$ and As fluorescence. with minimal attenuation of the In fluorescence. The polymer window supplied by the manufacturer is used to improve the detector efficiency in the range $2-30 \mathrm{keV}$. and results in a nearly "flat" response in this region. Silicon ribs (0.3 $\mathrm{mm}$ thick. $100 \mu \mathrm{m}$ apart. $20-2.5 \mu \mathrm{m}$ wide) are used to support the polymer under vacuum and provide tight collimation of the photons incident on the Ge element.

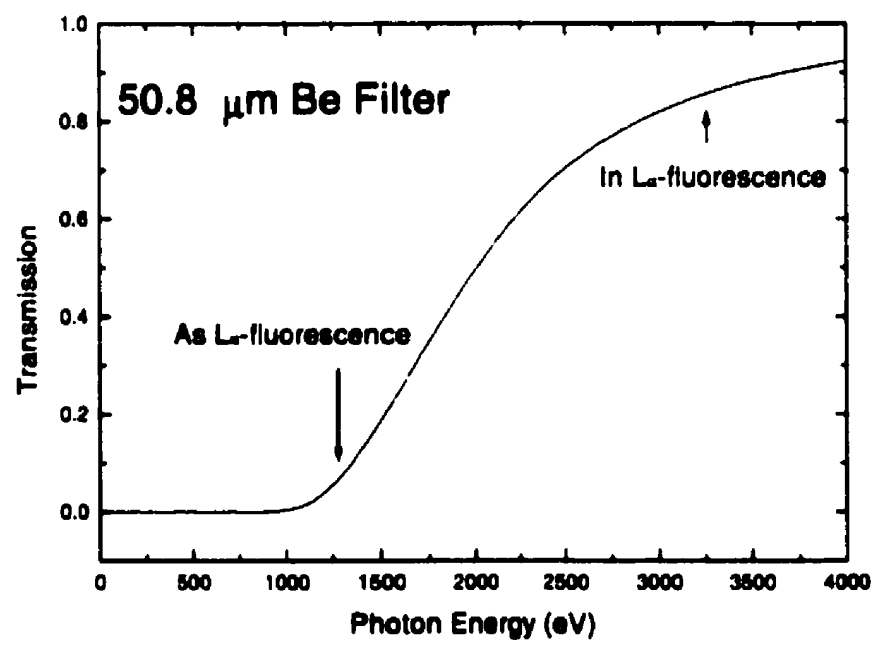

Figure 6.9: The transmission of X-Rays through a Be window of thickness $50.8 \mu \mathrm{m}$.

The detector signal from the preamplifier was passed through a cable transformer which reduces high frequency interference and into the amplifier in the nuclear instruments module (NIM) bin. This amplified analog signal was passed simultaneously to the analog-to-digital converter (ADC) and to a group of four single-channel analyzers (SCAs) in parallel (Figure 6.8). The ADC output was monitored by a multichannel analyzer (MCA).

The energy range monitored by the detector is determined by the applied bias 
voltage and gain set at the high-voltage power supply (HVPS). For these experiments the range extended from 0 to approximately $6000 \mathrm{eV}$. The ADC output to the MCA allowed the observation of the measured detector counts in real time. More importantly, the MCA was used to set the energy windows for the four SCAs as follows. Consider a typical MCA spectrum as illustrated in Figure 6.8. Four peaks were observed and associated with the following emission lines: Ga. As $\mathrm{L}_{\alpha, 3}$ : In $\mathrm{L}_{\alpha, 3}$ : and $X$-ray clastic scattering. This identification was based on a detector calibration obtained using an indium ingot mounted in the sample position. The fourth peak is related to the pulser reference signal and will be discussed presently.

By gating each SCA with the ADC signal individually, and in the coincidence counting mode. it was possible to isolate the energy window associated with the SCA for observation on the MCA. The energy level and the window width were then adjusted to the desired bandwidth. Thus the four SCAs were set to measure the In fluorescence: the total detector fluorescence: the Ga and As fluorescence: and the pulser signal.

The pulser reference signal to the detector "test" input was used to monitor the dead time of the detector. By supplying a regular signal with well-defined frequency and amplitude, it was possible to ensure that the detector did not become saturated at the GaAs(004) Bragg condition. when the number of fluorescence counts became quite large. Indeed. the pulser signal measured by the detector was observed to remain quite flat throughout the energy range of the experiments.

Each sample was aligned in the sample chamber by optimizing the sample position to find the back-reflection Bragg peak in the reflectivity and the XSW pattern in the TEY. For each sample approximately twenty scans were recorded, over a range of 4381 to $4391 \mathrm{eV}$ in steps of approximately $0.064 \mathrm{eV}$ and with a count time on the order of 5 seconds per step. The data were then summed together to obtain good statistics as described in Section 7.1 . In the next section the XAFS measurements at SSRL will be described. By combining the XSW and XRD measurements with XAFS, which studies the local environment of an absorbing atom, it is possible to develop a more complete structural understanding of the InAs SQWs. 


\subsection{Grazing Incidence XAFS}

This section describes the equipment and the methods which were employed in the XAFS measurements performed at SSRL. Following a description of the SSRL-designed grazing incidence X-Ray absorption spectroscopy (GIXAS) apparatus [132], the method of alignnent and dala acquisition will be vutlined. This includes buth reflectivity and XAFS measurements, which were performed in the polarization-dependent near-total reflection mode at the In Ki-edge $(27940 \mathrm{eV})$.

XAFS measurements were made at SSRL beamline $4-2$ with the storage ring operating at an electron energy of $3 \mathrm{GeV}$ and a stored current in the range of 100-65 $\mathrm{mA}$. Data were collected with a $\mathrm{Si}(220)$ double-crystal MC. with an entrance slit width of $1 \mathrm{~mm}$ and no beam focusing. (inder these conditions no detuning was used. All XAFS measurements described in this thesis were performed at room temperature.

A schematic of the GIXAS stage is given in Figure 6.10. The apparatus uses 18 motors interfaced to the beamline VAX system. Three motors on the rail. along with the hutch vertical table motion. were used for two-point alignment of the beam. Each Huber slit set consists of a pair of blades in both the vertical and horizontal directions. S1 and S2 were used for defining the incident beam. while S3 was used for alignment and during reflectivity scans. The sample position was controlled by three motors defining the grazing angle, $\theta:$ the azimuthal angle. $\phi$ : and the vertical position, z.

The $X$-ray intensity before and after the sample was measured using the ionization chambers, $I_{0}$ and $I_{1}$, respectively. These were filled with argon gas at a pressure slightly greater than $1 \mathrm{~atm}$. The currents were measured using picoammeters with a gain of $10^{10} \mathrm{amps} /$ Volt.

\begin{tabular}{|c|c|c|c|}
\hline \hline Element & $\boldsymbol{K}_{\alpha 1}[\mathrm{eV}]$ & $\boldsymbol{K}_{\alpha 2}[\mathrm{eV}]$ & $\boldsymbol{K}_{31}[\mathrm{eV}]$ \\
\hline $\mathrm{Ga}$ & $9251 . i 4$ & 9224.82 & 10264.2 \\
\hline $\mathrm{As}$ & 10543.72 & 10507.99 & 11726.2 \\
\hline $\mathrm{In}$ & 24209.7 & 24002.0 & 27275.9 \\
\hline
\end{tabular}

Table 6.3: X-Ray emission energies (from Bearden [131]). 


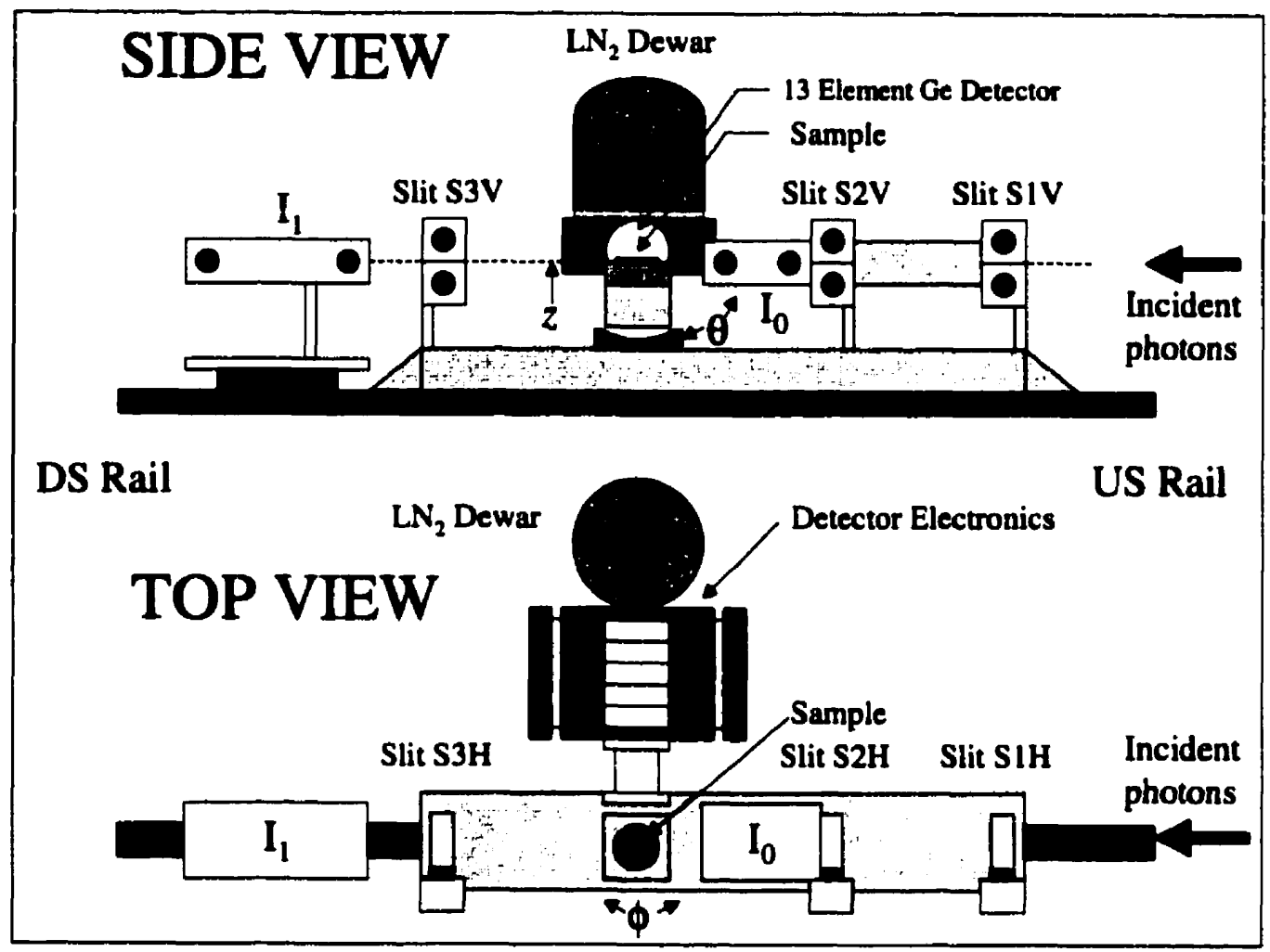

Figure 6.10: The SSRL GIXAS stage. The whole apparatus is rotated $90^{\circ}$ about an axis parallel to the beam path for measurements in the perpendicular polarization.

In $K_{\alpha}$-fluorescence excitation from the samples was measured using a 13 element Ge solid state detector. similar to the one used for XSW measurements but with a much larger active surface area. The electronics instrumentation for each detector element was equivalent to that used for the single element detector described in Section 6.6. To reduce the total incoming count rate signal from the $\mathrm{Ga}$ and As fluorescence, two Al filters of thickness $0.75 \mathrm{~mm}$ were placed in front of the detector. The energy dependence of the transmission is shown in Figure 6.11. From the figure it is apparent that the $\mathrm{Ga}$ and As fluorescence is strongly reduced by the filters. A guideline for the setup was to keep the total incoming count rate in each detector channel 
below about $3 \times 10^{4}$ counts/s. Bevond this level saturation of the detector becomes a concern. The energies of the $K$-fluorescence lines for the elements of interest are given in Table 6.3.

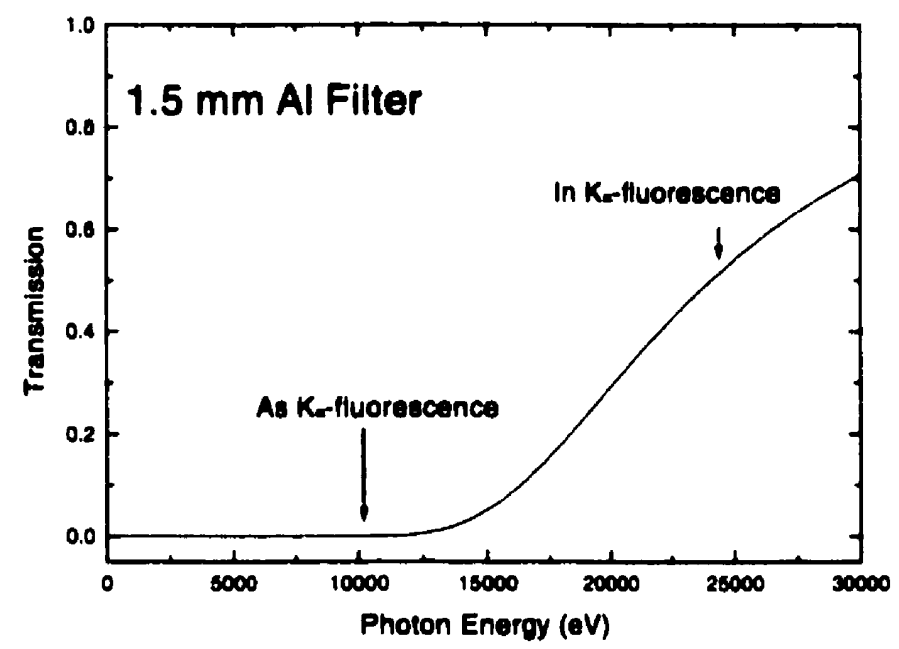

Figure 6.11: The transmission of $X$-rays through a $1.5 \mathrm{~mm}$ Al filter.

Alignment of the GIXAS stage with the beam was accomplished in a straightforward way using the upstream (US) and downstream (DS) rail motions. For the reflectivity and XAFS measurements the following procedure was used. The Huber slits were set to the desired aperture, which differed between the $E_{\|}$and $E_{\perp}$ configurations, due to the asymmetry of the beam footprint. The "vertical" beam height was limited by the sample size and geometry as will be discussed. The sample height. $z$, was increased until the $I_{1}$ beam intensity was equal to half of the full-beam value. indicating that the sample was eclipsing the beam. The glancing angle. $\theta$. was then optimized to its centre position. The sample height and angle were then optimized iteratively with respect to one another [132].

Figure 6.12 depicts the $\mathrm{X}$-Ray beam illuminating a sample in the GIXAS geometry. The samples measured in this thesis consisted of $1 / 4$ sections of a 2" diameter round 


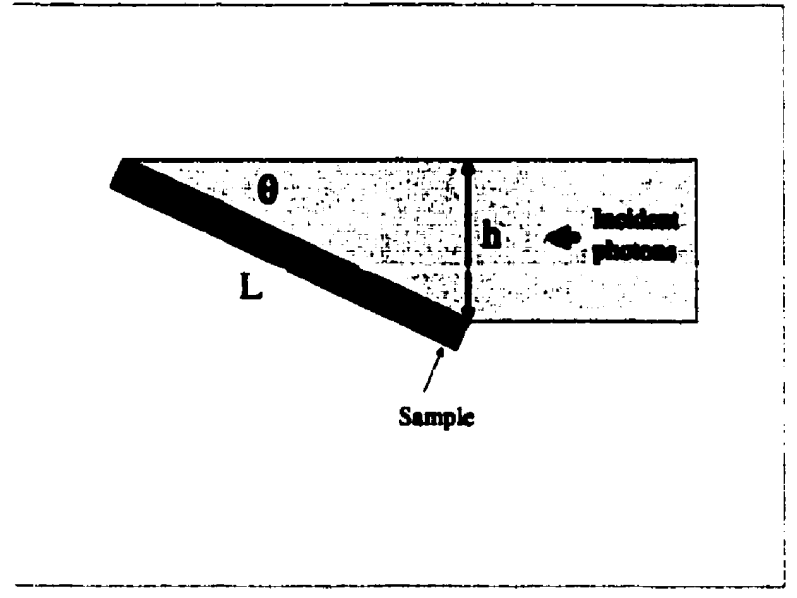

Figure 6.12: The experimental GIXAS geometry.

wafer. and so had a maximum length of approximately $L=25.4 \mathrm{~mm}$. From the geometry it is clear that a beam height of $h=L \sin \theta$ would be just sufficient to illuminate the whole sample surface. At $200 \mathrm{eV}$ above the In- $\mathrm{K}$ edge the critical angle is $\theta_{C}=90$ mdeg. for which the appropriate beam height is $40 \mu \mathrm{m}$. To study In layers buried beneath $\sim 100 \mathrm{~A}$ of GaAs it was necessary to make XAFS measurements at $\theta=220$ mdeg which required a correspondingly larger beam height. In most of the measurements we used $h=300 \mu \mathrm{m}$.

For the reflectivity geometry, the top vertical blade of $\mathrm{S} 33$ was raised to admit the reflected beam. With the sample lowered out of the beam path, the lower S3 blade was then raised to occlude the straight-through beam and the sample was returned to its optimum position. Reflectivity measurements were performed by scanning the glancing angle, while measuring the incident and reflected beam intensities and In li-fluorescence.

XAFS spectra were obtained by fixing the glancing angle and scanning in energy throughout a range which includes the In- $K$ edge. The measurement ranges are shown in Table 6.4. For each sample approximately 15 "sweeps" of this type were used, each of which contains fluorescence data measured by the 13 element detector, normalized 


\begin{tabular}{|c|c|c|}
\hline \hline Energy range $[\mathrm{eV}]$ & Stepsize $[\mathrm{eV}]$ & Count time $[\mathrm{sec}]$ \\
\hline $27740-27920$ & 10 & 1 \\
\hline $27920-27970$ & 1.06 & 3 \\
\hline $27970-28686.86$ & $1.08 \rightarrow 5.33$ & 3 \\
$\left(2.81 \AA^{-1}-14 \AA^{-1}\right)$ & $\left(0.05 \AA^{-1}\right)$ & \\
\hline
\end{tabular}

Table 6.4: The XAFS spectra were divided into three ranges. Coarse steps were used below the absorption edge. In the XANES region a finer mesh was used. In the EXAFS region the steps were defined in $k$-space, rather than in energy.

by the incident intensities. A technical nuisance which occurs in this type of measurement is the appearance of Bragg peaks in the XAFS spectra. By carefully observing the position of Bragg peaks in the XAFS spectra for each channel and rotating the o stage of the sample. it was possible to move these peaks to the extremes of the spectra and sometimes to remove them altogether. Still. analysis of the XAFS chata involves a lengthy process of "deglitching" the spectra measured by each of the detector elements. for all sweeps recorded for each sample. The sweeps are then summed together to provide good statistics.

In the next chapter the experimental data will be presented. The chapter will describe the growth sequences employed in the MOVPE sample preparation and the characterization of the samples in the SFU laboratory and at the other facilities described in the preceeding sections. Considerable attention will be given to the methods of data analysis and interpretation. 


\section{Chapter 7}

\section{Experimental Results}

In the previous chapter the experimental systems were described. This chapter will give the details of the sample preparation for the MOVPE and ALE layers grown at SFU and also for an XSW study of MBE samples. Several groups of samples were prepared at SFU and characterized, both in-situ using RDS and $c x-s i t u$ using HRXRD. PL, AFM, XSW and XAFS. These studies will be described separately and the main results will be presented. The chapter will begin by outlining the details of the XSW data analysis.

\subsection{Analysis of Experimental XSW Data}

\subsubsection{Preliminaries}

Before beginning the description of the XSW data analysis in InAs/GaAs samples, it is useful to consider results obtained using a $\operatorname{GaAs}(001)$ reference wafer. As described in Section 6.6, an XSW measurement for a single sample is obtained by taking multiple measurements and then summing the data to enhance the signal:noise and improve the experimental precision. A suite of $\mathrm{C}++$ programs designed for the $\mathrm{XSW}$ analysis handle the preliminary processing of the raw data and ultimately use nonlinear leastsquares fitting to determine the XSW coherent position, $P^{H}$, and coherent fraction, $F^{H}$, for the sample. Characteristic data after processing are shown in Figure 7.1 . 


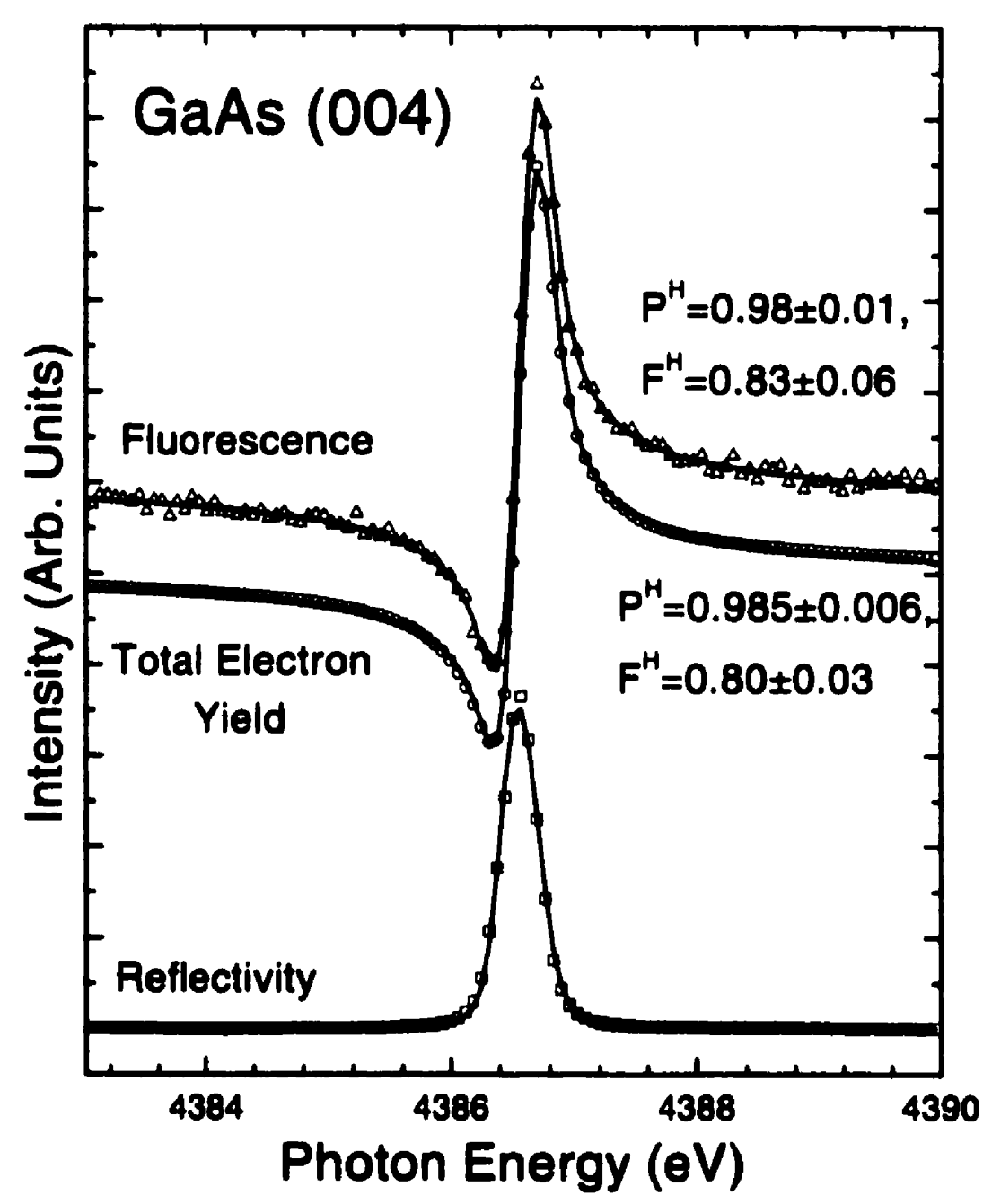

Figure 7.1: Photon energy dependence of the reflectivity (bottom curve) for a reference epi-ready $\mathrm{GaAs}$ crystal near the $\mathrm{GaAs}(004)$ Bragg backreflection condition. The XSW patterns obtained by measuring the total electron yield and the Ga and As-L fluorescence yields are shown in ascending order, with fit parameters as indicated. The solid lines are the best fits to the data points and the curves were offset for clarity. 
The figure shows the sum of a series of reflectivity scans for a bulk GaAs reference crystal near the (004) back-reflection condition. The semi-insulating (SI) substrate crystal was manufactured by American XTAL Technology (AXT) using the vertical gradient freeze (VGF) method. It is important to note that this epi-ready crystal was measured with no cleaning or processing. The XSW patterns were obtained using the total electron yield ( $\mathrm{TEY}^{\circ}$ ). and the $\mathrm{Ga}$ and As L-fluorescence yields (FLY). At the low energy side of the Bragg reflection the nodes of the XSW lie on the substrate planes. As the phase shifts throughout the Bragg peak the wavefield moves continuously until the antinodes lie on the substrate planes at the high energy side. The TEY and FLY spectra in Figure 7.1 are proportional to the absorption of the atoms in the field of the XSW and are used to deduce the spatial distribution of a given type of atom.

Raw datasets cannot be summed directly because the MC tends to exhibit a slight energy offset between consecutive scans. These offsets were accounted for using the following method. Each XSW dataset contains columns corresponding to the substrate reflectivity; the Ga and As FLY; the TEY; the In L-fluorescence (for samples containing In): the total FLY; and the pulser reference signal. For each scan the reflectivity. $R$. as a function of photon energy. $E$. was calculated using the DT.RRD as discussed in Chapter 3 . In this calculation the structure factors $F_{H}$ and $F_{A}$ were computed for the $(004)$ and $(000)$ GaAs substrate reflections using the method of Appendix A. Recall that for the symmetric Bragg case used in these measurements the polarization appearing in Equation 3.30 has the value $P=1$, while the asymmetry factor, $b=-1$. For the backreflection geometry (Equation 3.47)

$$
\eta=\frac{\left(-2.2 E / E+\Gamma F_{0}\right)}{\Gamma\left[F_{H} F_{\bar{H}}\right]^{1 / 2}}
$$

where $\Delta E=(E-\delta E)-E_{B}$, in terms of the MC energy offset. $\delta E$, and the Bragg energy, $E_{B}$. The reflectivity predicted by the DTXRD is then given by (Equation 3.33)

$$
R=\frac{\left|F_{H}\right|}{\left|F_{\bar{H}}\right|}|6|\left|\eta \pm\left(\eta^{2}-1\right)^{1 / 2}\right|^{2}
$$

The experimental data cannot be fitted to this simple expression because of the spectral broadening of the beamline apparatus. The Si(220) MC crystals each have 
a characteristic, finite, rocking curve which contributes to the energy spectrum of the "monochromatic" beam. To account for this divergence, the reflectivity given by Equation 7.2 is convoluted with a Gaussian which simulates the lineshape of the MC signal. (For a complete discussion of multiple crystal arrangements, see Reference [133].) The Gaussian has the form

$$
G(E)=\frac{1}{\sigma \sqrt{2 \pi}} \exp \left[\frac{-\left(E-E_{i}\right)^{2}}{2 \sigma^{2}}\right] .
$$

Here $\sigma$ is the standard deviation of the MC lineshape, while $E_{i}$ is the nominal energy in IC steps, and $E$ is the energy of a given point on the curve centred at $E_{i}$. The convolution is carried out by numerically integrating the convolution function $R^{\prime}(E)=$ $\int G^{\prime}\left(E^{\prime}\right) R\left(E+E^{\prime}\right) d E^{\prime}$ over a range of $E^{\prime}$ equivalent to about $16 \sigma$. In the fitting program $\sigma$ is a fitting parameter and the $\mathrm{MC}$ lineshape has a width $\mathrm{FWHM}=2.354 \sigma$. Experimental values of $\sigma$ near $0.09 \mathrm{eV}$ were found. corresponding to FWHMI=0.2l $\mathrm{eV}$.

The experimental reflectivity is then least-squares-fitted using the Marquardt algorithm [134] to the convoluted rocking curve. multiplied by a linear function of the form ( $\left.A+B\left(E-E_{1}\right)\right)$ to account for the variation in the beam flux with energy. $E_{1}$ is the energy of the first point in the scan. Thus, each reflectivity dataset is fitted with the variable parameters: $\delta E, \sigma . A, B$ and $C$ to the expression

$$
R_{f i t}(E)=\left(A+B\left(E-E_{1}\right)\right)\left(1+C^{\prime} R^{\prime}(E)\right) .
$$

The signal measured by the $\mathrm{Ni}$ mesh is actually proportional to $\left(1+\mathrm{C} R^{\prime}(E)\right)$ because the incident and reflected beams pass through the mesh simultaneously. The final fit parameter, $C$, accounts for the intensity loss of the reflected beam in travelling from the crystal to the mesh.

Each single-scan reflectivity curve was fitted in this way and corrected to remove the energy offset. A 4th order polynomial interpolation [134] was then used to put the data back on their original "grid" in energy space. The other data (TEY. etc.) obtained in each scan were corrected using the same $\delta E$. Repeating this process for all scan datasets allowed the datasets to be added properly, without introducing significant systematic errors. To eliminate artifacts from the interpolation the energy range of the data was reduced by $1 \mathrm{eV}$ at each extreme. 
Figure 7.1 shows the reflectivity, the TEY and the FLY data for the reference GaAs crystal after following this procedure to remove the offsets and to sum the scans. A few points are noteworthy at this stage. The reflectivity curve does not exhibit the asymmetric Darwin-Prins shape (Figure 3.3) because of the convolution with the MC resolution function. The convolution of two or more crystal rocking curves generally produces this shape [13:3]. Secondly, the TEY and FLY curves are very well-defined. The random scatter associated with a measurement of $N$ counts produces a statistical uncertainty. $\sqrt{N}$, in the measured values, about the mean value. From the figure the uncertainty may be roughly estimated by the scatter in the points. suggesting that the FLY spectum has a quite low uncertainty $\left(\sqrt{N} / . \sim 1.3 \%\right.$ at $\left.E_{1}\right)$. The (ia and As FLY signals have poorer statistics than the In FLY signals used to measure the SQW samples because of lower counting times. and because of attenuation of the lower-energy signals by the Be filter (Section (6.6). The TEY signal exhibits no such scatter whatsoever. and has very good statistics $\left(\sqrt{.} / N \sim 0.4 \%\right.$ at $\left.E_{1}\right)$. In general. data were collected for sufficient times to allow a maximum statistical uncertainty in the In FLY of approximately $1 \%$.

\subsubsection{Standing Wave Analysis}

After offset correction and the summation of a series of datasets the experimental data may be fitted to an expression describing the XSW. The reflectivity sum is first fitted using the same method as in the preceeding section to determine the level of any remaining offset, $\delta E$ and the energy resolution parameter, $\sigma$. The fit parameters $A, B$ and $C$ are useful for linear background removal and normalization (this has been done for all of the data presented here). The offset and resolution are more important because they affect not only the reflectivity, but the other measured data as well.

The TEY or FLY XSW curves are fitted in the following way. The offset in the XSW data is corrected using the energy offset determined by the reflectivity fitting. The Gaussian broadening function is similarly calculated using the appropriate value of $\sigma$. Using Equation 3.53 the XSW yield is given by

$$
Y^{H}(E)=1+R(E)+2 \sqrt{R(E)} F^{H} \cos \left(\alpha(E)-2 \pi P^{H}\right),
$$


with the fit parameters: $P^{H}$. which measures the coherent position of a particular type of atom relative to the substrate planes, normalized by the diffraction plane spacing, $d_{s}$, and the coherent fraction. $F^{H}$. which measures the $\delta$-like nature of the atomic distribution perpendicular to the diffracting planes. The reflectivity, $R(E)$ and phase, $\alpha(E)$ of the substrate reflection are determined in the usual way. I'his expression is convoluted with the ciaussian function as before, i.e. $Y^{\prime H}(E)=\int G^{\prime}\left(E^{\prime}\right) Y^{H}\left(E+E^{\prime}\right) d E^{\prime}$. Finally, the experimental data are leastsquares-fitted to the convoluted $\mathrm{XSW}$, multiplied by a linear function of the form $\left(A+B\left(E-E_{1}\right)\right)$ to account for the variation in the beam flux with energy. Again $E_{1}$ is the energy of the first point in the scan. Thus, each . XSW dataset is fitted with the variable parameters: $A, B, P^{H}$ and $F^{H}$ to the expression

$$
\left.Y_{f i t}^{\cdot H}(E)=\left(A+B\left(E-E_{1}\right)\right) Y^{-1 H}(E)\right) .
$$

The error bars in the fit parameters $P^{H}$ and $F^{H}$ were determined using the residual sum of squares, defined as

$$
\sum(s q)=\sum_{i=1}^{N}\left(y_{i}^{f i t}-y_{i}\right)^{2}
$$

where $y_{i}$ is the measured value of the $i$ th data point and $y_{i}^{\text {fit }}$ is the corresponding best-fit value. $V$ is the number of data points. The quoted uncertainties indicate the values which double the squared residuals in each parameter while all other values were allowed to float.

Figure $\mathbf{7} .1$ shows the fitting results for both the TEY and FLY signals measured for the reference crystal. For this sample the expected values are $P^{H}=1.0$ (corresponding to atoms situated on the (004)-planes), and $F^{H}=\epsilon^{-M}$, which is the Debye-Waller factor ( 0.9) in GaAs [34]. The measured values (Figure 7.1 ) obtained using both types of emission (TEY, FLY) agree with one another and are close to the expected values. Lee et al. used the evanescent wave effect to measure a GaAs buffer laver underneath a single buried InAs layer in GaAs [4]. Their measured values of $P^{H}=$ $1.00 \pm 0.01$ and $F^{H}=0.81 \pm 0.01$ are in good agreement with the present results. The depth sensitivity of the fluorescent photons is discussed in Reference [4]. In the 
experiments described in this thesis, the average takeoff angle viewed by the detector aperture was greater than the critical angle for fluorescent X-Rays leaving the crystal $\left(1.5^{\circ}\right.$ at the $A s-L_{\alpha_{1}}$ energy, $\left.1282 \mathrm{eV}\right)$. with relatively little extinction, and so the FLY includes contributions from atoms at depths of hundreds of angstroms below the surface. Also. for the relatively low photon energies employed in this work the TEY signal should approximate a bulk site close to the crystal surface [135]. and so the measured values are due to atoms on bulk sites within the crystal. This suggests that the Debye-Waller factor may be slightly smaller than 0.9 in these samples.

With this background in XSW analysis we now consider the XSW measurements of InAs SQW' samples.

\subsection{XSW Study of MBE-Grown Films}

The first series of samples measured using XSW as part of this thesis work were studied in collaboration with NIST and Brooklyn College. NY in order to extend the structural understanding of InAs SQWs which had begun to form through earlier measurements [2. 3. 4]. These MBE films were prepared by Dr. Joseph Pellegrino and Dr. Joseph Woicik at NIST and the XSW measurements were carried out at X2.4.

The MBE samples were grown on AXT SI GaAs (001) nominally-exact substrates which were chemically cleaned and then etched using a conventional $\mathrm{H}_{2} \mathrm{SO}_{4}-\mathrm{H}_{2} \mathrm{O}_{2}$ deionized $\mathrm{H}_{2} \mathrm{O}$ solution. After desorbing the surface oxide laver in the $\mathrm{MBE}$ chamber at $580{ }^{\circ} \mathrm{C}$ in the presence of an As flux. a GaAs buffer layer. $0.5 \mu \mathrm{m}$ thick. was grown using conventional solid sources of $\mathrm{Ga}$ and $A$ s with effusion cell technology. Throughout the MBE growth the substrates were rotated at a rate of $8 \mathrm{rpm}$.

In a similar fashion to that described elsewhere [2, 3], 1 and 1/2 ML samples were grown using a "temperature ramp" technique. During the buffer layer growth the temperature was linearly ramped to $480{ }^{\circ} \mathrm{C}$ and the growth was interrupted for $3 \mathrm{sec}$ with the As shutter open before the growth of the InAs layer. The GaAs and InAs growth rates were determined by RHEED oscillations to be $1.059 \mathrm{sec} / \mathrm{ML}$ and $5.04 \mathrm{sec} / \mathrm{ML}$ in reference samples with no substrate rotation. The InAs layer growth 
was followed by another $3 \mathrm{sec}$ interruption, after which a 200 A GaAs cap was deposited at $480^{\circ} \mathrm{C}$. An additional series of samples were grown in which the buffer. $1 \mathrm{ML}$ InAs and $200 \AA$ GaAs cap layers were grown at a fixed temperature of 4:30, 480, 530 or $580{ }^{\circ} \mathrm{C}$. The XSW results for both types of samples are shown in Figures $1: 2$ and 7.3 . Note that the reflectivity curves, arising from Bragg reflection in the substrate crystals, are essentially identical for all samples. Thus a single experimental reflectivity curve is shown in each plot as a reference.

\begin{tabular}{|c|c|c|c|}
\hline \hline Description & $P^{H}$ & $F^{H}$ & In-As planar distance (A) \\
\hline I ML (MBE) [1] & $1.17 \pm 0.02$ & $0.58 \pm 0.07$ & $1.65 \pm 0.03$ \\
\hline I ML (MBE) [2.,3] & $1.16 \pm 0.02$ & $0.73 \pm 0.1$ & $1.64 \pm 0.03$ \\
\hline $1 \mathrm{ML}(\mathrm{MBE})[4]$ & $1.154 \pm 0.01$ & $0.43 \pm 0.03$ & $1.63 \pm 0.01$ \\
\hline I ML $\operatorname{In}_{0}{ }_{18} \mathrm{Ga}_{0.52} \mathrm{As}(\mathrm{MBE})[3]$ & $1.053 \pm 0.02$ & $0.53 \pm 0.1$ & $1.49 \pm 0.03$ \\
\hline \hline
\end{tabular}

Table 7.l: XSW results reported from MBE-grown InAs and $\ln _{x} \mathrm{Ca}_{1-x}$ As films in GaAs $(001)$, with references indicated. The In-As planar distances were calculated from the measured coherent positions.

Results of previous XSW measurements for these types of samples are sumnarized in Table 7.1. Recall that the MET predicts the position of the In atoms to be $P^{H}=1.1496$, relative to the GaAs (004)-planes. Our 1 ML sample grown by the temperature ramp technique (Figure 7.2 ) should be similar to the sample which was measured using back-reflection XSW $[2,3]$ and conventional XSW at $6 \mathrm{keV}[4]$. The samples were prepared in the same MBE chamber under similar conditions, although the previous sample was deposited on a stationary substrate with simultaneous RHEED measurements. Also, the previous sample had only a $25 A$ GaAs cap, and the slower InAs growth rate of $23.5 \mathrm{sec} / \mathrm{ML}$. These latter differences are not expected to change the sample quality. The sample rotation may be important, however, because the growth rates determined by RHEED during stationary MBE growth are almost certainly different from the growth rates obtained with a rotating substrate. It is possible that higher InAs growth rates in the rotating case could result in a degradation of the InAs layer quality if the InAs layer thickness exceeds $1 \mathrm{ML}$. In samples containing 2 and $4 \mathrm{ML}$ of InAs, Woicik et al. [3] found that the coherent position is larger for samples with more InAs, which rules out this possibility. Alternatively. 


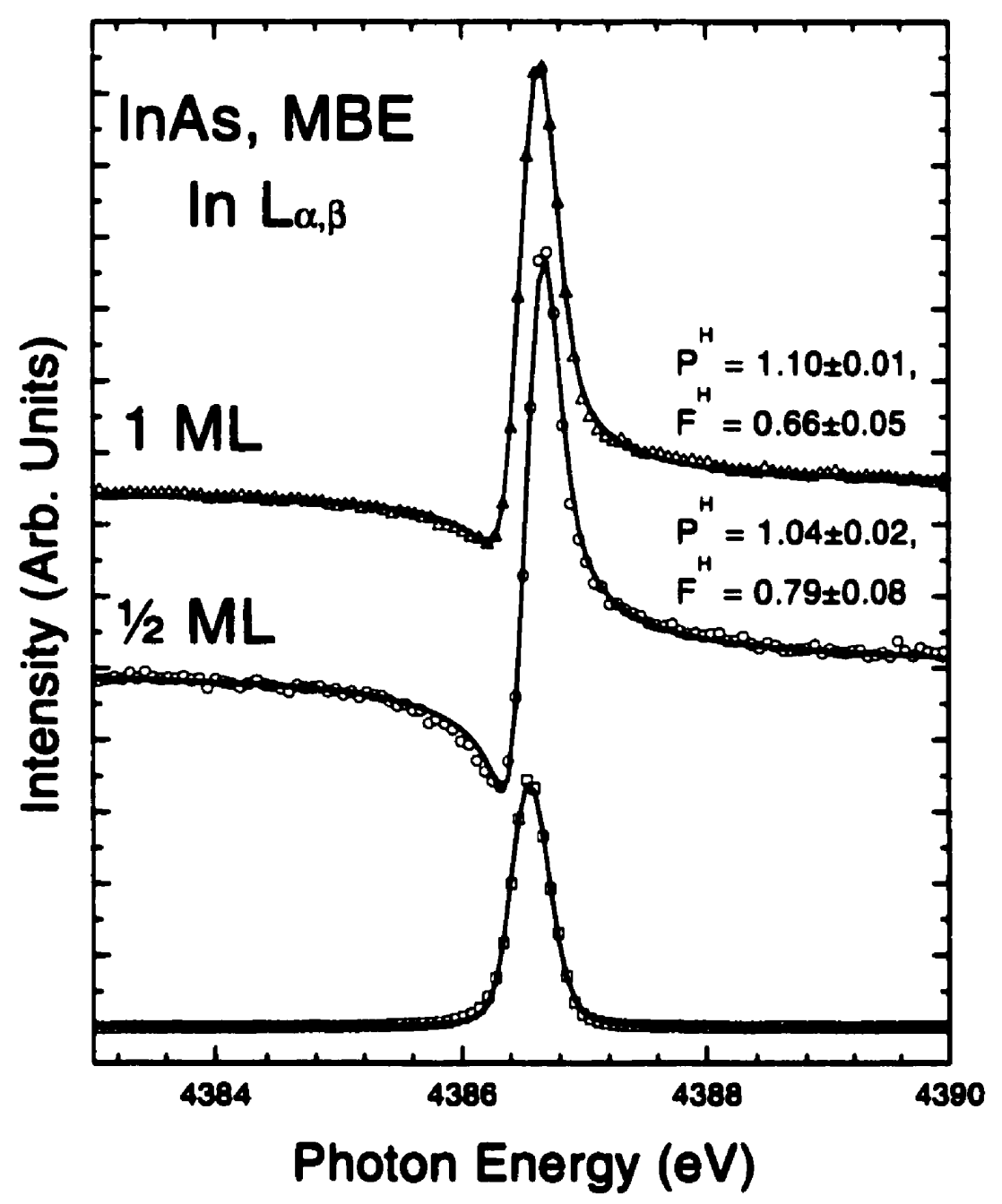

Figure i.2: Photon energy dependence of the reflectivity (bottom curve) and the In-L fluorescence yield near the GaAs(004) Bragg backreflection condition for 1 and $1 / 2$ ML InAs samples grown by MBE using the temperature ramp technique. The solid lines are the best fits to the data points and the curves were offset for clarity. 


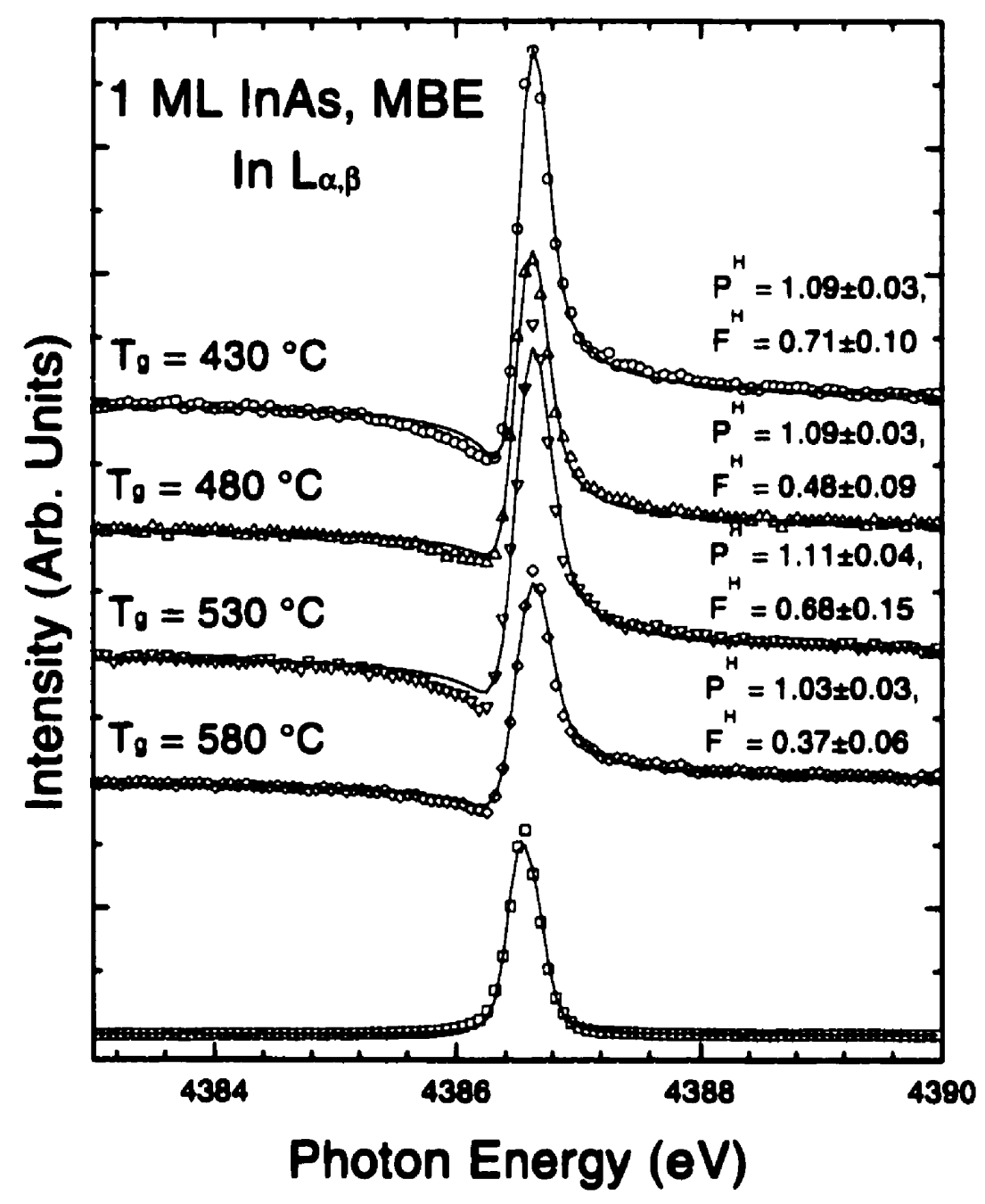

Figure i.3: Photon energy dependence of the reflectivity (bottom curve) and the In-L fluorescence yield near the $\mathrm{GaAs}(004)$ Bragg backreflection condition for a series of $1 \mathrm{ML}$ InAs samples grown by MBE, with growth temperatures as indicated. The solid lines are the best fits to the data points and the curves were offset for clarity. 
lower In As growth rates would result in the incorporation of less than one complete ML of material. Referring to Figure 7.2 and Table 7.1 it appears that the 1 ML temperature ramp sample is not as highly strained as the previous sample, according to the lower value of $P^{H}$. This would be consistent with less than one complete ML of In atoms. At the same time the relatively high value of $F^{H}$ suggests good InAs layer quality, comparable to the earlier sample. The value of $P^{H}$ obtained for the $1 / 2 \mathrm{ML}$ sample is comparable to the value for the $\operatorname{In}_{x} \mathrm{Ga}_{1-x}$. As layer studied in Reference [3]. while $F^{H}$ for the present sample is significantly higher.

In order to predict the XSW coherent position for a sample with less than 1 ML of InAs, we use the MET. For In atoms occupying random sites in a thin $\operatorname{In}_{r} \mathrm{Gia}_{1-r}$.As layer. Vegard's law gives the unstrained lattice constant of the laver. $a_{f}$, as

$$
a_{f}=x a_{l n, A s}+(1-x) a_{G u, A s} .
$$

The elastic constants for the alloyed layer are given by the concentration-weighted values for InAs and GaAs [5;3], as

$$
\begin{aligned}
& C_{11}^{f}=x C_{11}^{l n A s}+(1-x) C_{11}^{G a \cdot 4 s} . \\
& C_{12}^{f}=x C_{12}^{l n A s}+(1-x) C_{12}^{G(j u, 4 s} .
\end{aligned} .
$$

From MET

$$
a_{\perp}=a_{f}\left(\epsilon_{\perp}+1\right) .
$$

or

$$
a_{\perp}=a_{f}-2\left[\frac{x C_{12}^{l n A s}+(1-x) C_{12}^{G u A s}}{x C_{11}^{\ln A s}+(1-x) C_{11}^{G u A s}}\right]\left(a_{G u . A s}-a_{f}\right) .
$$

If the In concentration, $x$, is known then $P^{H}$ is determined from $P^{H}=a_{\perp} / a_{\text {GaAs }}$. Otherwise, from an experimentally measured $P^{H}$ the value of $x$ can be determined by substituting equation $i .8$ into equation $i .11$, and using the $\mathrm{XSW}$ result $a_{\perp}=P^{H} a_{G a A s}$. For the samples measured in the present study, the corresponding values of $x$ are shown in Table 7.2. When this approach is applied to the results of Woicik et al. [3], in which $\mathrm{XSW}$ measurements were made for a single $\operatorname{In}_{x} \mathrm{Ga}_{1-x}$ As layer (nominally $x=0.48$ ), it is found that the measured $P^{H}=1.053 \pm 0.02$ is indicative of an alloy composition $x=0.38 \pm 0.14$. This is quite reasonable, since the input $\mathrm{Ga}:$ In ratio and growth rates were determined from growths with much thicker layers. 
It is apparent from Table 7.2 that these MBE-grown samples do not contain complete In atomic planes residing in well-defined positions. The low value of $P^{H}$ for the 1 ML temperature ramp sample might be explained by an InAs growth rate of only $69 \%$ of that required to deposit a complete ML. For the 1/2 ML the corresponding value is $54 \%$.

\begin{tabular}{|c|c|c|c|}
\hline \hline Description & $P^{H}$ & $F^{H}$ & $x$ \\
\hline 1 ML (temperature ramp) & $1.10 \pm 0.01$ & $0.66 \pm 0.05$ & $0.69 \pm 0.07$ \\
\hline 1/2 ML (temperature ramp) & $1.04 \pm 0.02$ & $0.79 \pm 0.08$ & $0.27 \pm 0.14$ \\
\hline 1 ML $\left(430^{\circ} \mathrm{C}\right)$ & $1.09 \pm 0.03$ & $0.71 \pm 0.10$ & $0.62 \pm 0.21$ \\
\hline 1 ML $\left(480^{\circ} \mathrm{C}\right)$ & $1.09 \pm 0.03$ & $0.48 \pm 0.09$ & $0.62 \pm 0.21$ \\
\hline 1 ML $\left(530^{\circ} \mathrm{C}\right)$ & $1.11 \pm 0.04$ & $0.68 \pm 0.15$ & $0.75 \pm 0.27$ \\
\hline 1 ML $\left(580^{\circ} \mathrm{C}\right)$ & $1.03 \pm 0.03$ & $0.37 \pm 0.06$ & $0.21 \pm 0.2 \mathrm{~L}$ \\
\hline \hline
\end{tabular}

Table 7.2: XSW results and the In concentration, $x$. determined from experimentally measured values of $P^{H}$ for $\mathrm{MBE}$-grown InAs and $\operatorname{In}_{r} \mathrm{Ga}_{1-r}$. As films in GaAs(001).

The lack of any clear $T_{y}$-dependence is due to the competing temperature requirements for growth of the different lavers in each sample. Higher temperatures are most suitable for producing smooth, uniform Ga.ts buffer layers, but are the least suitable for minimizing In segregation, according to the discussion of Section 5.2. Conversely. lower temperatures reduce the efficiency of the In/ Ga exchange during the GaAs cap layer growth but result in poor buffer layer quality. This suggests that high-quality GaAs buffer layers must be carefully prepared and then suitable temperatures should be selected for the InAs layer and cap layer growth to produce good material. The following sections will describe the sample preparation and XSW results for ALE films.

\subsection{XSW Study of ALE-Grown Films: I}

In order to study the relationship between the measured XSW structural parameters and the PL optical parameters, InAs SQW samples were first produced by "ALE" using a growth sequence which had previously resulted in good PL emission from samples with several InAs coverages [5]. For this study the samples were grown at 50 
Torr on SI VGF GaAs(001) epi-ready substrates, used with no additional cleaning. Following annealing under TBAs at $580^{\circ} \mathrm{C}$ for $300 \mathrm{sec}$, a GaAs buffer layer of thickness $0.625 \mu \mathrm{m}$ was grown by conventional MOVPE using TEGia and TBAs. ALE growth was performed at $390^{\circ} \mathrm{C}$. CaAs barrier layers (of thickness $\sim 1.3 \mathrm{ML}$ ) above and below the InAs layer were grown by ALE in four 4-step cycles each consisting of a 3 sec $\mathrm{H}_{2}$ purge, 8 sec of TMGa, another $3 \mathrm{sec} \mathrm{H}_{2}$ purge. and finally $6 \mathrm{sec}$ of TBAs. The InAs layer was deposited in a single cycle as above. with a $3 \mathrm{sec}$ or $1.5 \mathrm{sec}$ TMIn pulse. for the single and half MLs, respectively. The $\ln A$ s growth rate was based on XRD thickness measurements of In.As homoepitaxial films deposited by ALE. After the top GaAs ALE layer, the temperature was raised to $500^{\circ} \mathrm{C}$ and a thicker MOVPE GiaAs layer was deposited using TEGia and TBAs. It should be emphasized that the CaAs "ALE" sequences were not optimized to produce exactly $1 \mathrm{ML} / \mathrm{cycle}$.

In this study three samples were produced. For the XRD and XSW measurements, two samples were grown with nominal In depositions of 1 ML and 1/2 ML. and CiaAs caps (ALE+MOVPE) of approximately $100 \mathrm{~A}$. Actual cap thicknesses were determined by XRD, as will be discussed. A third sample was grown for PL characterization with $1 \mathrm{ML}$ of InAs and a total GaAs cap thickness of 2550125 A. also determined by XRD. During each growth the sample surface was monitored by RDS.

The XSW results are shown in Figure 7.4 . Comparing the 1 ML results with the MBE results reported previously. it appears that the ALE sample is not as highly strained. The coherent fraction of $0.7 i \pm 0.05$ is anong the best measured, but still lower than the Debye-Waller factor. For the half ML sample the $F^{H}$-value of $0.88 \pm 0.12$ corresponds to about 0.98 after accounting for the thermal effects.

Figure 7.5 shows a $4.2 \mathrm{~K}$ PL spectrum for the nominally I ML sample with the thicker GaAs cap. The peak location $(1.459 \mathrm{eV})$ is close to the expected position for excitonic emission from an InAs ML in GaAs (Table 5.2). while the linewidth of $5.74 \pm 0.01 \mathrm{meV}$ is as narrow as the best results reported elsewhere and is indicative of satisfactory growth conditions. This spectrum was obtained using a double-grating $0.85 \mathrm{~m}$ spectrometer and GaAs photocathode photomultiplier tube, rather than the Bomem system. 


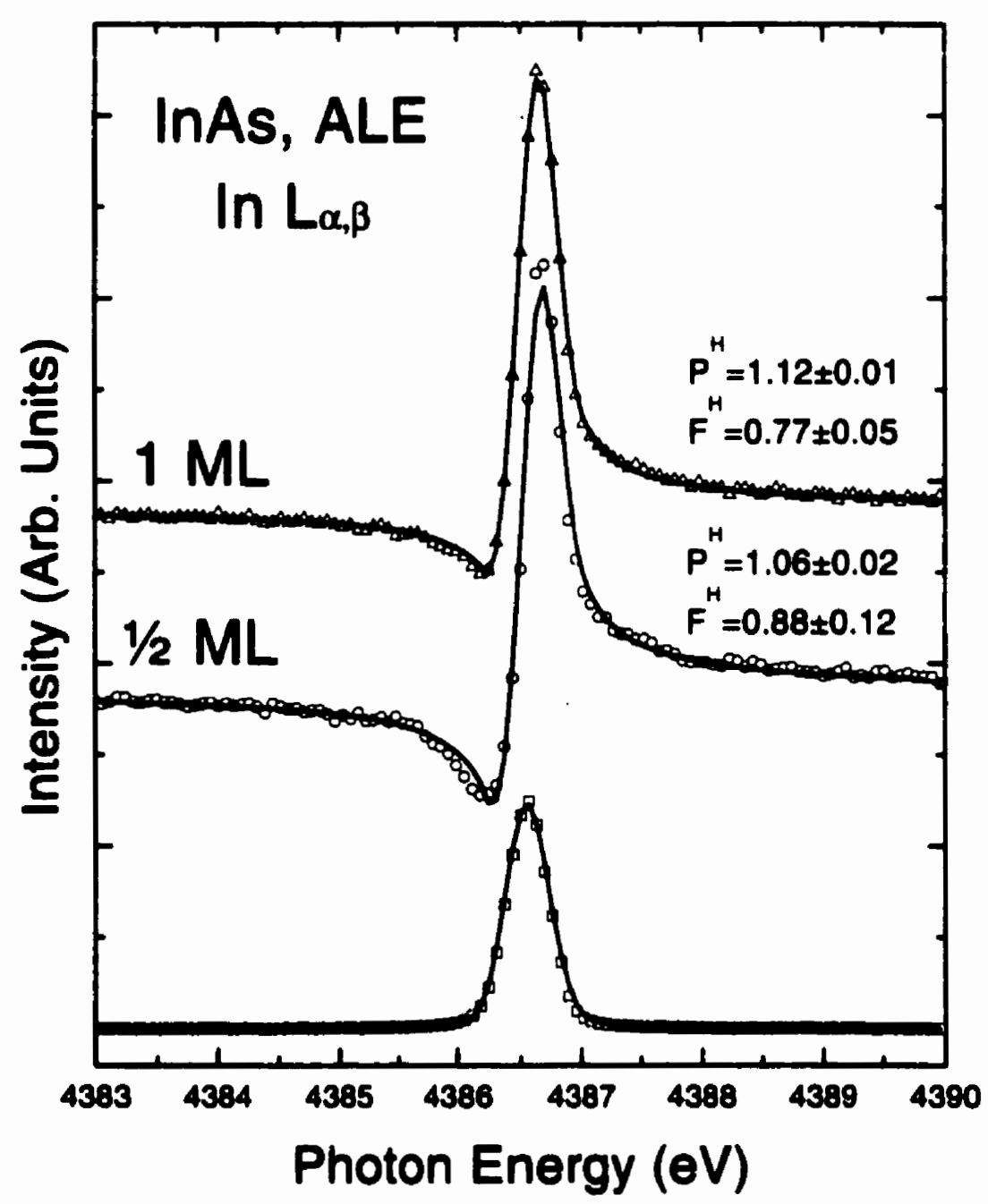

Figure 7.4: Photon energy dependence of the reflectivity (bottom curve) and the In-L fluorescence yield near the GaAs(004) Bragg backreflection condition for $\perp$ and 1/2 ML InAs samples grown by ALE. The solid lines are the best fits to the data points and the curves were offset for clarity. 


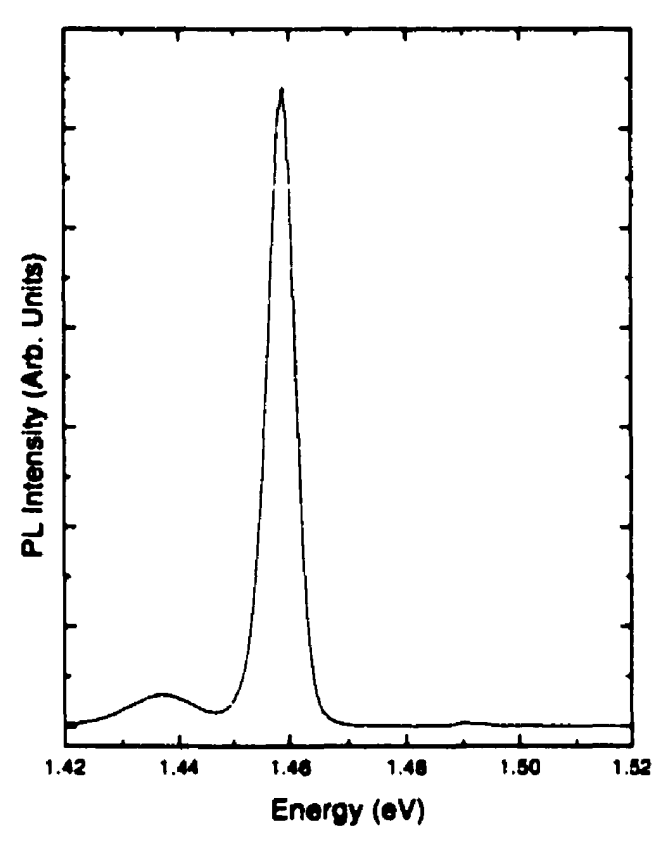

Figure 7.5: PL emission from a nominally 1 ML InAs film on GaAs(001), buried beneath a $2550 \pm 25 \mathrm{~A}$ GaAs cap.

What is the significance of the XSW positional measurements? Previous measurements of MBE samples support the macroscopic description of this interface $[1]-[3]$. Our results may be used to predict the layer composition in a similar fashion to the XRD determination of alloy concentration by the measurement of lattice constant in thicker films [136].

Assume that the XSW position is correct, and that the In atoms are arranged in a single layer. Based on the experimental result this layer cannot be completely covered by $I n$. If the layer actually consists of an alloyed distribution, $\operatorname{In}_{x} \mathrm{Ca}_{1-r} \cdot \mathrm{As}$, then $x$ can be found from the MET using the method of Section 7.2 . The measured values of $a_{\perp}=6.31 \pm 0.06 \AA$ and $a_{\perp}=6.01 \pm 0.12 \AA$ lead to $x=0.79 \pm 0.07$ and 
$x=0.45 \pm 0.15$, for the $1 \mathrm{ML}$ and $1 / 2 \mathrm{ML}$ films, respectively. For the MBE halfML samples discussed previously the alloyed distribution of cations is to be expected because the In and $\mathrm{Ga}$ shutters were open simultaneously during the MBE growth. In contrast. for the ALE films no Ga-source molecules were incident on the substrate during the InAs layer growth.

Figure 7.6 shows the XRD data for the $1 \mathrm{ML}$ and $1 / 2 \mathrm{ML}$ samples. It is interesting to note that the Pendellösung fringes are clearly visible out to 7200 arcsec on either side of the main substrate reflection. This is somewhat remarkable for such thin layers of InAs capped by nominally $100 \mathrm{~A}$ of GaAs. In HRXRD studies of thin InAs layers reported previously [1. 13]. the GaAs caps typically exceeded $\sim 275 \mathrm{~A}$.

The XRD data were modeled using the RADS software (Section :3.3). The XSW data discussion has suggested that the $\mathrm{In}$ atoms reside in single $\mathrm{In}_{r} \mathrm{Ga}_{1-r}$. As layers. and that the group III layer has a distance $a_{\perp} / 4$ from the last $A$ s plane of the substrate. Thus the net GaAs cap $\rightarrow$ substrate displacement is $a_{\perp} / 2$.

Figure 7.6 shows the results of simulations with these alloy compositions and laver thicknesses. In addition, the GaAs cap thicknesses determined from the simulations are $97 \pm 3 \mathrm{~A}$ for the $1 \mathrm{ML}$ sample. and $85 \pm 3 \mathrm{~A}$ for the $1 / 2$.ML sample. Comparing the data and the simulations, it appears that layer properties inferred from the XSW results provide a good description of the XRD data. It is important to mention that the XRD data could be interpreted differently. For instance, a reasonable simulation for the 1 ML sample rocking curve could have been obtained by supposing a single $\operatorname{InAs}$ layer and an associated cap $\rightarrow$ substrate displacement of approximately $3.0 \pm 0.3$ A. although this can not be related to a physical distribution of In atoms.

Two factors may contribute to the apparently incomplete coverage in the ML sample. Without the benefit of RHEED growth oscillations, the ALE process is at a disadvantage compared with MBE. Specifically, the In pulse times were based on InAs bulk growth rates and we rely upon ex-situ XRD characterization to estimate the amount of In deposited. The In coverage can not be determined sufficiently precisely to distinguish between a full ML and a $79 \%$ alloy layer with this method. Secondly. the growth process employed the flash-off technique (Section 5.2). Raising the growth temperature after the GaAs cap ALE cycles may result in a greater-than-intended 


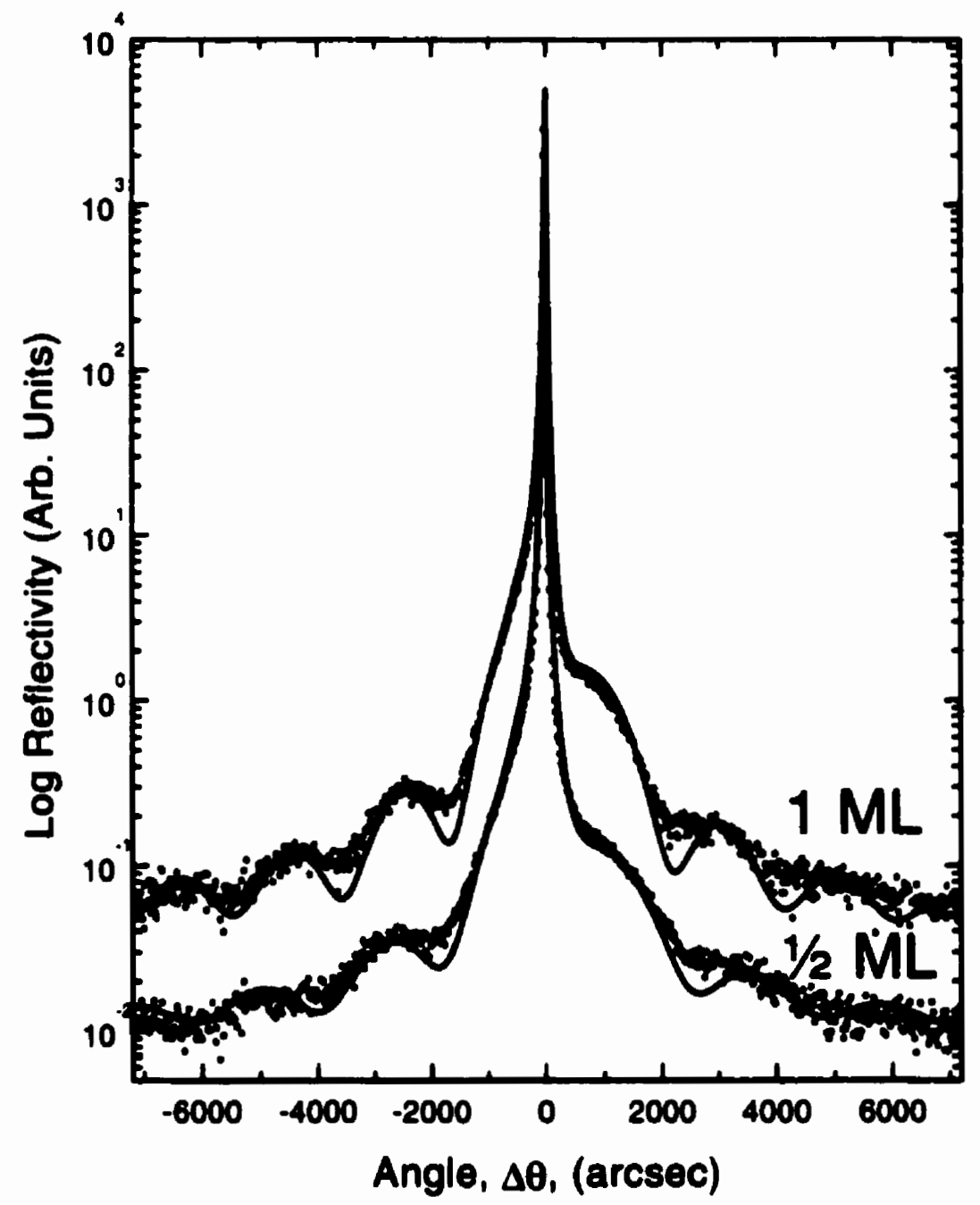

Figure 7.6: X-Ray rocking curve data obtained near the GaAs(004) symmetric substrate reflection, for $\Delta \theta=\theta-\theta_{B}$. Data for the 1 and 1/2 ML samples (upper and lower curves, respectively) are indicated by points. Solid lines indicate the results of RADS simulations as described in the text. 
desorption of segregated In atoms, thus contributing to the lower In coverage.

This interpretation of an alloyed distribution of In atoms, particularly in the 1/2 ML case, suggests that for subML, coverages the In atoms do not form ML islands, as proposed in the past $[13,119,121]$. Ideally. such islands would reside at the ideallystrained InAs position (1.624i $\AA$ ), although edge effects at InAs islands might lower the measured position. We must emphasize that this interpretation is not unique and unambiguous. In segregation could be involved, although calculations suggest that segregation alone cannot explain the low values of $P^{H}$ measured in these samples. The calculations will be discussed in Section 7.6.4. The next section will describe experiments aimed at studying the effect of growth conditions on In segregation in ALE.

\subsection{RDS Study of ALE Heteroepitaxy}

The RDS transient signal monitored at the As-dimer energy ( $2.6 \mathrm{eV})$ is quite sensitive to the presence and orientation of As dimers along the surface of a GaAs $(001)$ crystal. To extend the study published by Arès which clearly showed In segregation during ALE growth of $\mathrm{GaAs} / \mathrm{InAs} / \mathrm{GaAs}$ heterostructures [90]. attempts were made to reduce or eliminate the segregation by suitable modifications in the growth parameters.

The ALE growth sequence described in Section 7.3, although clearly suitable for producing material with good PL emission. does not make use of true "atomic layer" conditions. In the following the method for establishing the ALE $1 \mathrm{ML} / \mathrm{cycle}$ conditions for GaAs growth will be described. The temperature dependence of In segregation was explored for growth temperatures between 400 and $500{ }^{\circ} \mathrm{C}$.

The sample preparation began by annealing each sample under TBAs for $5 \mathrm{~min}$ at $580^{\circ} \mathrm{C}$. followed by the MOVPE growth of a $\sim 1450 \AA$ GaAs buffer at $580^{\circ} \mathrm{C}$ using TEGa and TBAs with flow rates of $8.07 \mu \mathrm{mol} / \mathrm{min}$ and $198 \mu \mathrm{mol} / \mathrm{min}$, respectively. All samples were grown on AXT SI GaAs(001) VGF epi-ready substrates with no additional cleaning. ALE growth was performed with TMGa at $25.7 \mu \mathrm{mol} / \mathrm{min}$, TMIn at $1.65 \mu \mathrm{mol} / \mathrm{min}$ and TBAs at $48.5 \mu \mathrm{mol} / \mathrm{min}$. The total reactor flows and gas velocities for ALE and MOVPE were given in Table 6.1. 


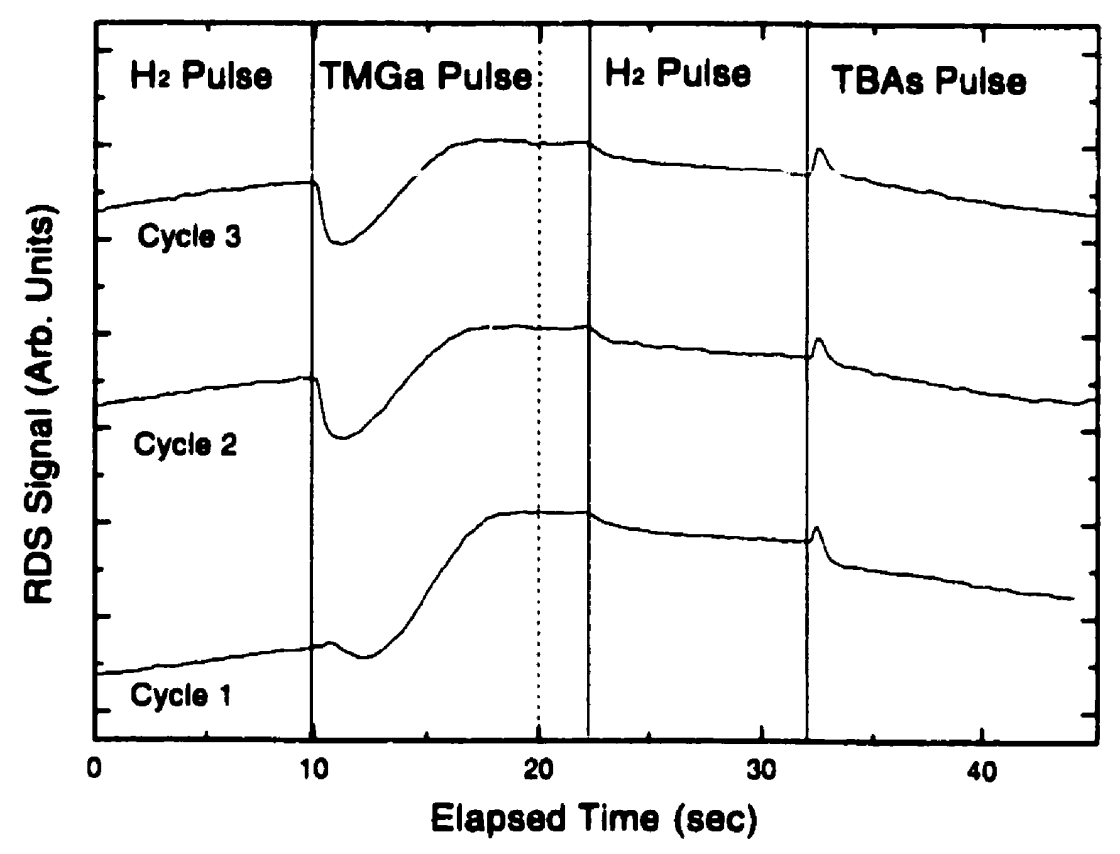

Figure $7 . \bar{i}$ : RDS transient signal during three consecutive GaAs ALE cycles at $400^{\circ} \mathrm{C}$. The dashed, vertical line marks the signal level after 10 sec of TMGa. The data were offset for clarity.

To determine the alkyl pulse durations required to produce $1 \mathrm{ML} /$ cycle at different temperatures, a single substrate crystal was used. For temperatures greater than $400^{\circ} \mathrm{C}$ the ALE sequence: 10 sec $\mathrm{H}_{2} \| \tau$ sec TMGa\|l $10 \mathrm{sec} \mathrm{H}_{2} \| \tau$ sec TBAs was repeated three times, while monitoring the $2.6 \mathrm{eV}$ RDS transient. At $400^{\circ} \mathrm{C} .12 \mathrm{sec}$ of TMGa and TBAs were used because of the slower growth and decomposition rates at this temperature. Figure $i . i$ shows the RDS transients for the three ALE cycles at $400{ }^{\circ} \mathrm{C}$, which are clearly reproducible from cycle-to-cycle. During the TMGa pulse the RDS signal saturates after an initial abrupt change. This saturation reflects the self-limiting nature of the growth and corresponds to a 1 ML Ga surface coverage. 
From similar RDS signals observed at the other temperatures it was possible to determine the TMGa pulse lengths required to produce $1 \mathrm{ML}$ of GaAs. The durations of the $\mathrm{H}_{2}$ and TBAs pulses are less critical. The $\mathrm{H}_{2}$ pulse is used mainly to separate the group III and group V pulses in time. The TBAs pulse must be sufficient to provide $1 \mathrm{ML}$ of As coverage. but excess As-adsorption does not increase the amount of GaAs deposited [16].

At higher $T_{g}$ the TMGa saturation was less well-defined because the ALE process was no longer self-limiting. In these cases the saturation time was estimated from the RDS transients and confirmed by growing samples with 1 ML of InAs capped by 200 GaAs ALE cycles. HRXRD determinations of the cap thicknesses were used to ensure that the growth sequences produced close to $1 \mathrm{ML} / \mathrm{cycle}$. The ALE sequences used for the remainder of this study consisted of: $2 \mathrm{sec} \mathrm{H}_{2} \| t \sec$ T.MGia\|l|2 $\sec \mathrm{H}_{2} \| 6 \mathrm{sec}$ TBAs. Here $t$ is the TMGia pulse time according to Table 7.3 . The InAs cycle used a 3 sec TMIn pulse.

\begin{tabular}{|c|c|}
\hline \hline Growth Temperature $\left({ }^{\circ} \mathrm{C}\right)$ & T.MGa Pulse Time. $l$ (sec) \\
\hline 400 & 10 \\
\hline 420 & 6 \\
\hline 440 & 3.5 \\
\hline 460 & 2 \\
\hline 480 & 1.6 \\
\hline 500 & 1 \\
\hline \hline
\end{tabular}

Table 7.3: TMGa pulse times required for $1 \mathrm{MLL} / \mathrm{cycle}$ ALE growth at different temperatures.

After establishing the appropriate TMGa durations. samples were produced at temperatures between 400 and $500{ }^{\circ} \mathrm{C}$. Each consisted of a Ga.As buffer layer. 10 GaAs ALE cycles, 1 InAs ALE cycle, 35 GaAs ALE cycles, and finally $2900 \AA$ of GaAs grown by conventional MOVPE using the buffer layer growth parameters. The additional cap layer was added to allow PL measurements. Throughout each sample growth the RDS transient was monitored at $2.6 \mathrm{eV}$. Unfortunately, the PL spectra for this series of samples were dominated by recombination at carbon impurities, preventing the observation of In excitonic emission. Apparently the amount of carbon 
incorporated in the $46 \mathrm{ALE}$ cycles is excessive. This illustrates a major shortcoming of the ALE technique.

A phenomenological analysis of the RDS data was performed in the following way. For each cycle. it is reasonable to assume that the most stable surface during the cycle will be achieved at the end of the last step, the TBAs pulse. To quantitatively study the transient signal, the points in each RDS transient corresponding to the end-ofcycle signal level were isolated. This allowed an examination of the transient signal as a function of the number of layers deposited, rather than as a function of time. This alleviates the complication that ALE cycles for different temperatures have different durations, as required for $1 \mathrm{ML} / \mathrm{sec}$ GaAs growth. An RDS spectrum obtained in this way is shown in Figure 7.8 .

For the temperatures considered here, the RDS signal prior to the InAs cycle was highly reproducible from cycle-to-cycle and the end-of-cycle levels were essentially constant during the GaAs ALE. Immediately following the InAs cycle, the RDS signal underwent a dramatic change in intensity and the end-of-cycle data followed a sigmoidal relaxation back to the original intensity. In Figure 7.9 the end-of-cycle data for samples grown with 1 ML GaAs ALE cycles at different temperatures are presented.

The solid lines in this figure are the results of least-squares-fitting the data to a purely empirical expression with a Boltzmann form

$$
I(n)=\left[1+\exp \left(\frac{n-n_{0}}{N}\right)\right]^{-1} .
$$

Here $I(n)$ refers to the normalized RDS signal as a function of the number of GaAs cap cycles deposited, $n$. The quantity $n_{0}$ is related to the half-width of the relaxation. while $\mathcal{N}$ is related to the decay rate of the segregation process.

The fits to the data using this expression appear quite good. The RDS half-widths decrease smoothly with decreasing growth temperature as shown in Figure $\mathrm{i} .10$ and may saturate at a minimum near $400{ }^{\circ} \mathrm{C}$. A more extensive study would be necessary to clarify the temperature dependence of these parameters. The most important observation is that using these ALE sequences, there remains considerable In segregation even at $400{ }^{\circ} \mathrm{C}$. 


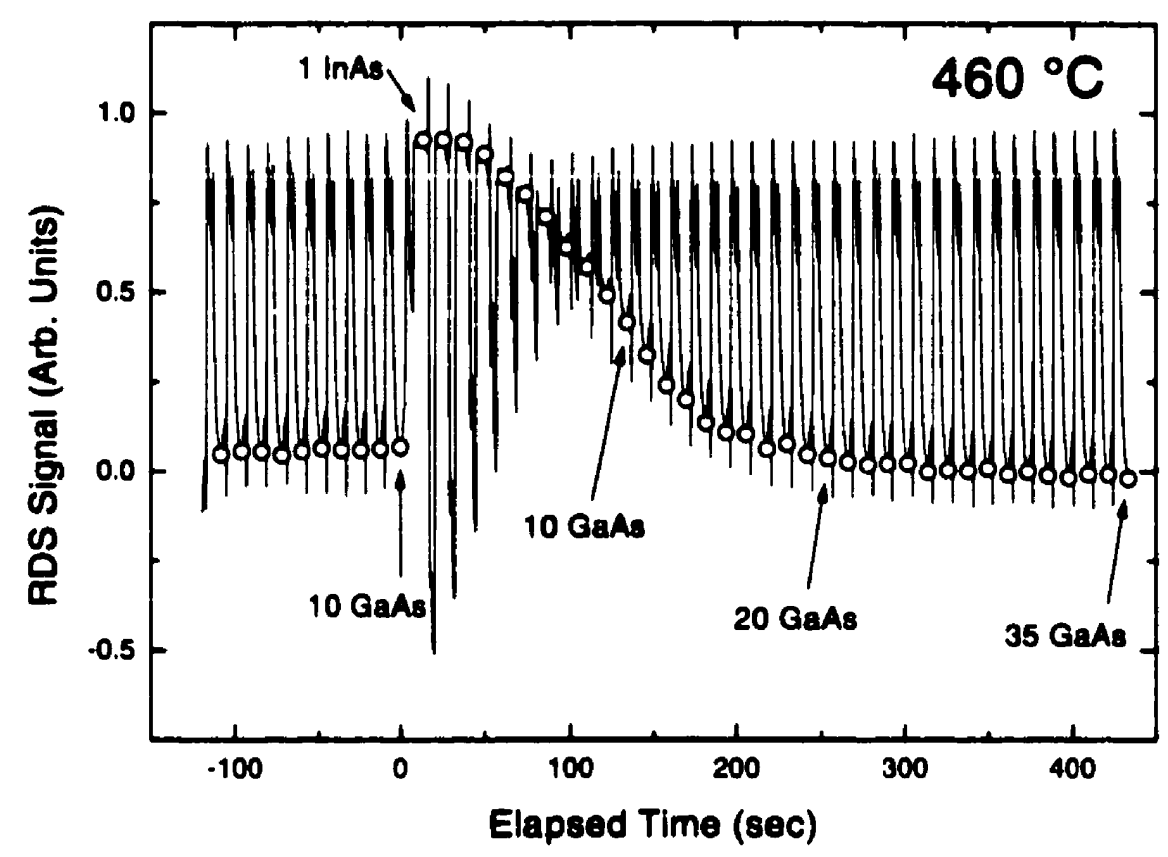

Figure 7.8: RDS transient signal during the deposition of 10 GaAs ALE cycles: 1 InAs cycle: and $35 \mathrm{GaAs}$ capping cycles at $460^{\circ} \mathrm{C}$. The empty circles indicate the position of the signal at the end of each complete cycle.

Based on the discussion of Section 5.2, the relaxation would be expected to decay exponentially, according to Equation 5. 7 . The In surface concentration after $n$ capping layers, $x_{n}$, in this equation follows the form

$$
x_{n}=(1-\sigma) \sigma^{n-1}=(1-\sigma) e^{(n-1) \ln \sigma} .
$$

where $\sigma$ is the segregation coefficient. The sigmoidal shape may indicate a more complicated process. Also, the RDS end-of-cycle level is not necessarily a linear function of the In surface concentration so the connection with Equation 7.13 may not be transparent.

Efforts were made to reduce the In segregation by adjusting the TMGa pulse times 


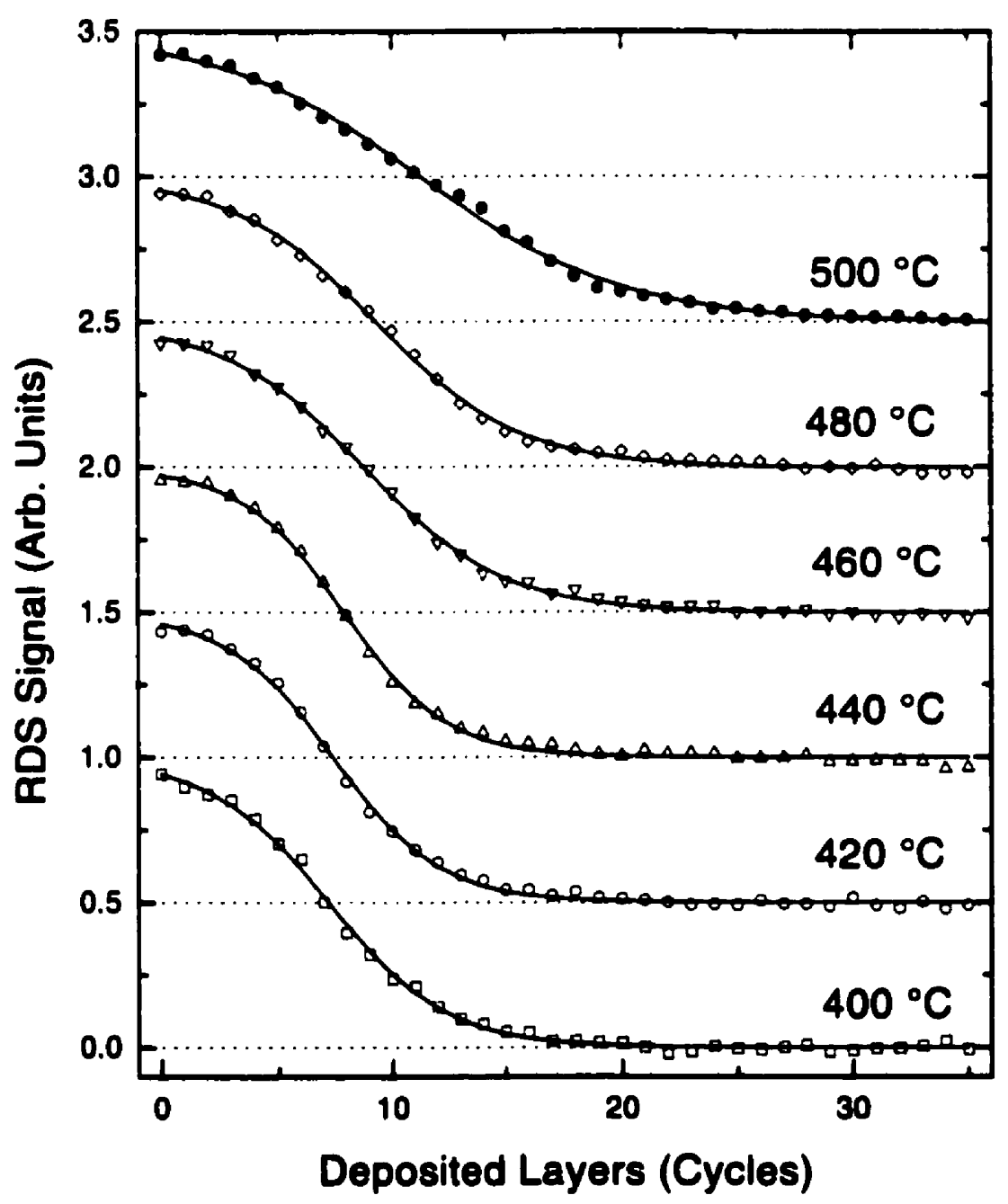

Figure 7.9: Temperature dependence of the RDS end-of-cycle transients during the deposition of $35 \mathrm{GaAs}$ capping layers by ALE. The solid lines indicate the fits using the Boltzmann expression. The curves were normalized and offset for clarity. 


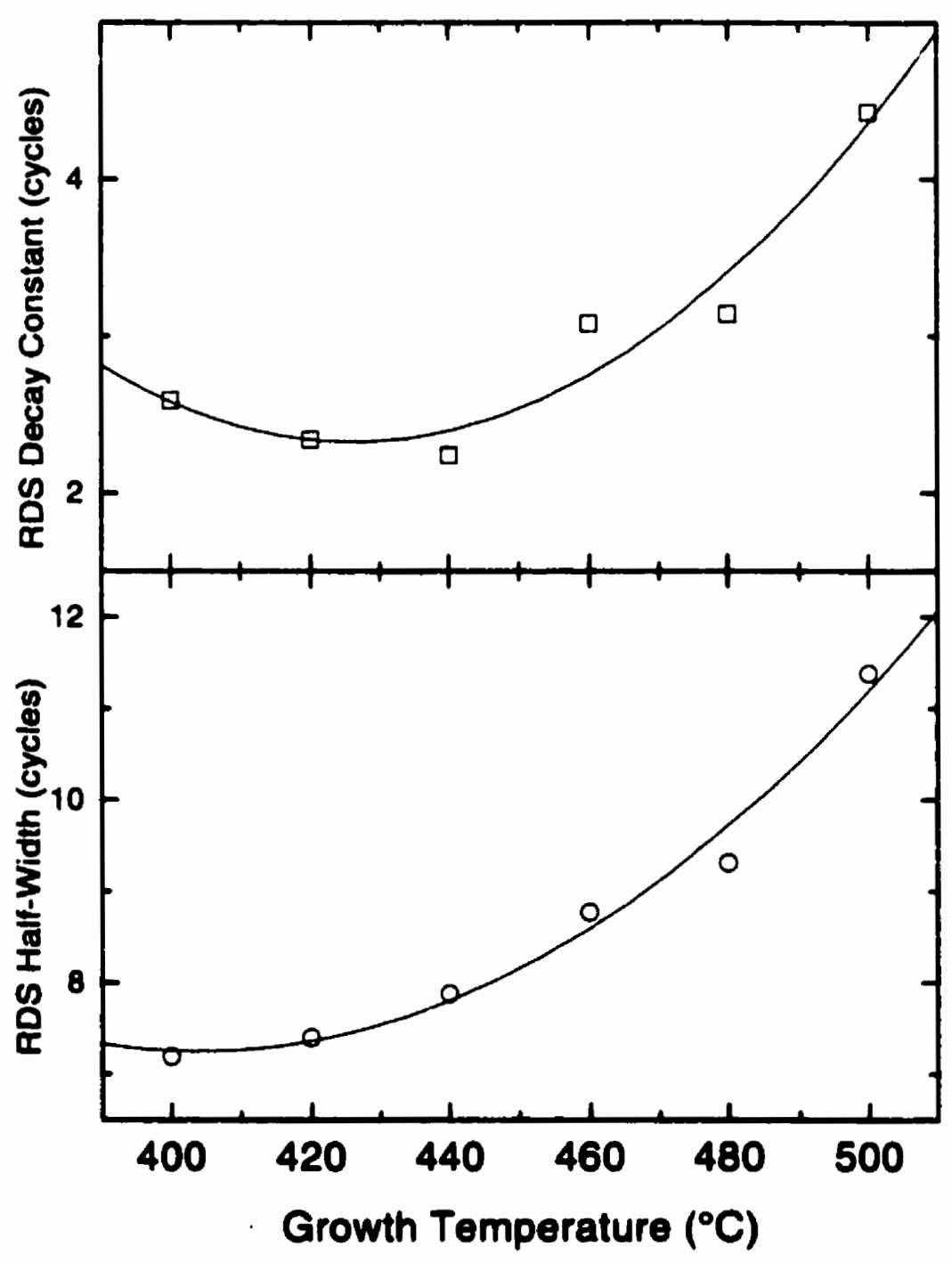

Figure 7.10: Temperature dependence of the RDS end-of-cycle fit parameters from the Boltzmann model. The solid lines are guides for the eye. 


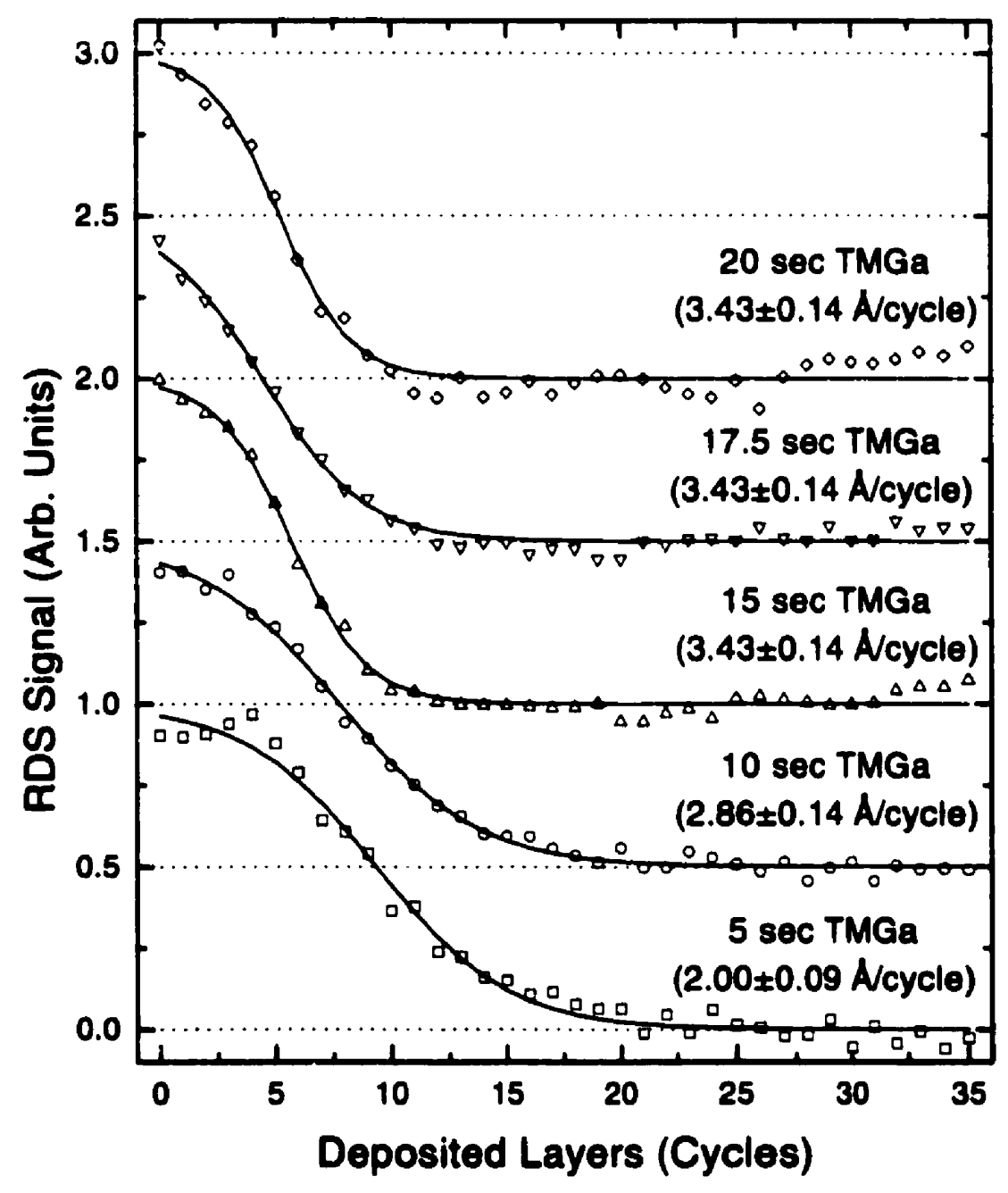

Figure 7.11: TMGa pulse time dependence of the RDS end-of-cycle transients during the deposition of $35 \mathrm{GaAs}$ capping layers by ALE. The solid lines indicate the fits using the Boltzmann model. The curves were normalized and offset for clarity. The values in parentheses indicate the growth rates as determined by ex-situ XRD. 
at $400{ }^{\circ} \mathrm{C}$. The results are shown in Figure 7.11 . At first glance it appears that the segregation is slightly reduced with the longest TMGa pulses. The amount of GaAs deposited per cycle is slightly different in each case. however. and so the segregation is not changed significantly as the TMGa pulse length is changed. Section 5.2 suggests that the ALE process is unsuitable for producing abrupt interfaces and this is verified by these experiments. The lack of $A$ s overpressure during the cap growth allows In segregation regardless of the temperature or TMGa pulse duration. The TMGa flow rate could play a role, but this was not pursued. The next section discusses the XSW results obtained for a SQW structure grown by ALE using the methods described in this section.

\subsection{XSW Study of ALE-Grown Films: II}

To compare the XSW results of Section 7.3 with the case of an ALE sample with true $1 \mathrm{ML} / \mathrm{cycle}$ GaAs growth. an ALE sample was produced at $400^{\circ} \mathrm{C}$ using the sequence: $2 \sec \mathrm{H}_{2} \| 10 \mathrm{sec}$ TMGa\|2 $\mathrm{sec} \mathrm{H}_{2} \| 6$ sec TBAs for GaAs growth. For InAs growth a $3 \mathrm{sec}$ TMIn pulse was used. Figure 7.12 shows the XSW data and the fitting results. For this sample also. $P^{H}$ has a lower value than expected for a perfect In.As laver $\left(P^{H}=1.1496\right)$ and $F^{H}$ is slightly lower than for the $1 \mathrm{ML}$ sample shown in Figure $\mathbf{7}$.4. Using the method of Section 7.2 , the data are consistent with In atoms arranged in a single $\operatorname{In}_{x} \mathrm{Ga}_{1-x}$ As layer with $x=0.89 \pm 0.14$. While this interpretation of the data is possible. it neglects the In segregation directly observed using RDS. As mentioned earlier, calculations accounting for the segregation process cannot adequately describe these data. Details of the calculations will be discussed in Section 7.6.4. At any rate, the ALE process is not expected to be the most suitable method for producing sharp InAs distributions. The next section involves the study of InAs films in CiaAs grown by conventional MOVPE, which forms the bulk of this thesis. 


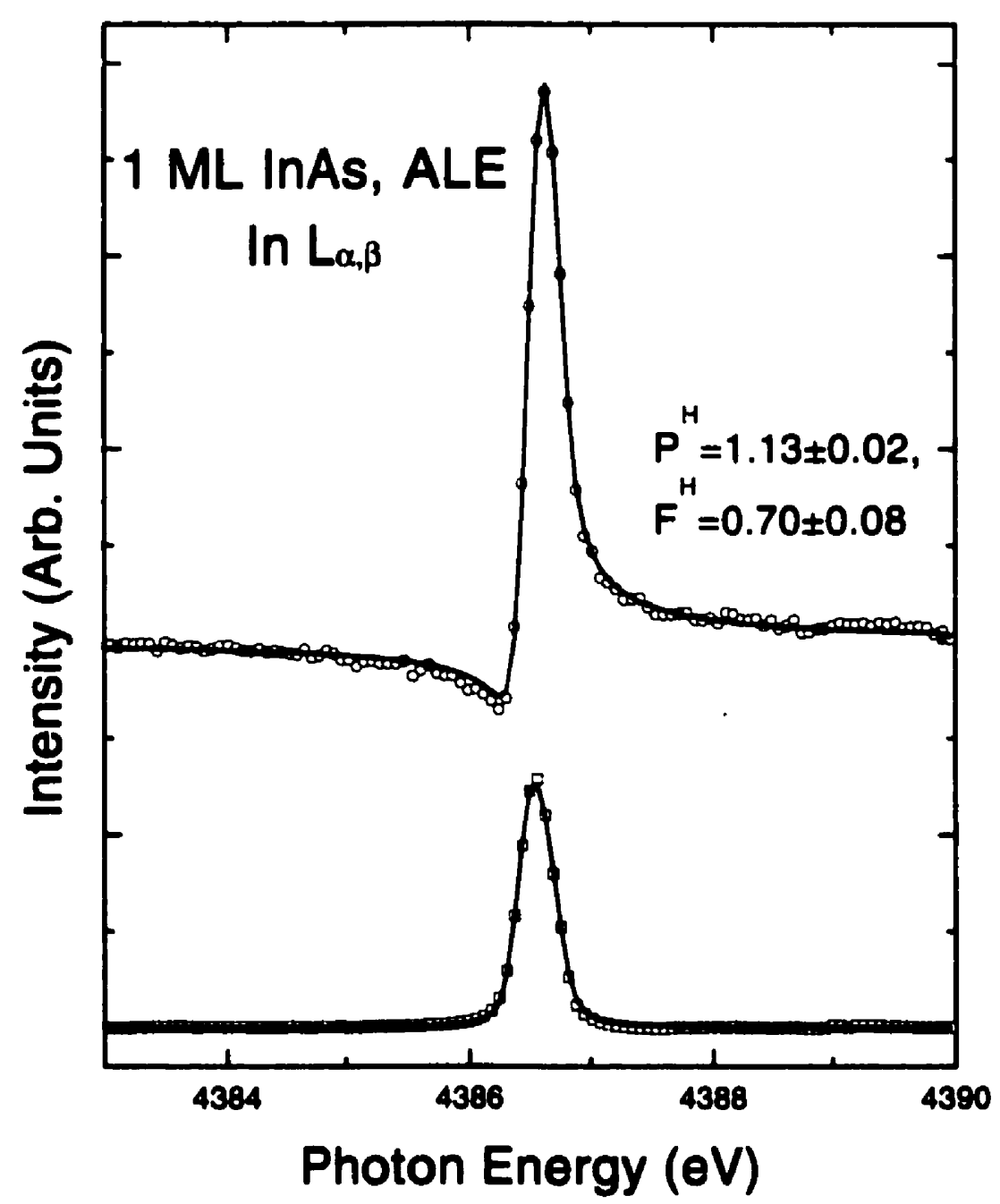

Figure 7.12: Photon energy dependence of the reflectivity (bottom curve) and the In- $\mathrm{L}$ fluorescence yield near the $\mathrm{GaAs}(004)$ Bragg backreflection condition for a $1 \mathrm{ML}$ InAs sample grown by ALE at $400{ }^{\circ} \mathrm{C}$. The solid lines are the best fits to the data points. 


\subsection{InAs QWs in GaAs(001) Grown by MOVPE}

This section describes the growth and characterization of the main group of samples studied in this thesis. First, RDS was used to perform a pseudo-real-time investigation of the GaAs/InAs/GaAs growth sequence using SL structures. Samples produced using single cycles of this sequence were then studied by XSW. XAFS and XRD. Additionally, a group of similar samples was examined by PL spectroscopy.

\subsubsection{MOVPE Growth and In-situ Characterization}

This series of samples was grown at 50 Torr using TBAs. TEGia, and TMIn with flow rates of 198.8 .07 . and $1.98 \mu \mathrm{mol} / \mathrm{min}$. respectively. All samples were grown on AXT SI GaAs(001) VGF epi-ready substrates with no additional cleaning. In all cases the substrates were annealed at $580{ }^{\circ} \mathrm{C}$ under TBAs, followed by the growth of a 1450 A GaAs buffer layer using TEGa and TBAs (hereafter called a standard buffer). AFM images of buffer layers grown in this way revealed excellent morphologies. with clear evidence of step-flow growth and terraces about $0.1 \mu \mathrm{m}$ wide. as shown in Figure 7.13.

Samples were produced at temperatures between 450 and $600{ }^{\circ} \mathrm{C}$. For the multitransient spectroscopy of the InAs QW growth. each sample was allowed to equilibrate at the growth temperature, $T_{g}$, under TBAs. The growth sequence for the SL structure was then initiated. Each cycle consisted of a $20 \mathrm{sec}$ pause under TBAs: $3 \mathrm{sec}$ of InAs growth; a second TBAs pause; and $100 \mathrm{~A}$ of GaAs growth. The GaAs growth rates determined by HRXRD are given in Table $i .4$. Here the growth rates were determined by growing a GaAs buffer layer, and then depositing $1 \mathrm{ML}$ of InAs and a reasonably thick ( $2000 \AA)$ GaAs cap layer at $T_{g}$. HRXRD data for calibration samples produced in this way were evaluated using the RADS software to estimate the GaAs cap thickness and hence the growth rate, based on the cap growth duration.

The 2.6 eV RDS transient signal was initially studied during the growth of 10 SL periods with a sampling frequency of $8 \mathrm{~Hz}$. During these cycles the RDS signal followed an oscillatory envelope, similar to the Fabry-Pèrot behaviour reported by Koch et al. [105]. After these ten cycles the signal stabilized enough to commence the 


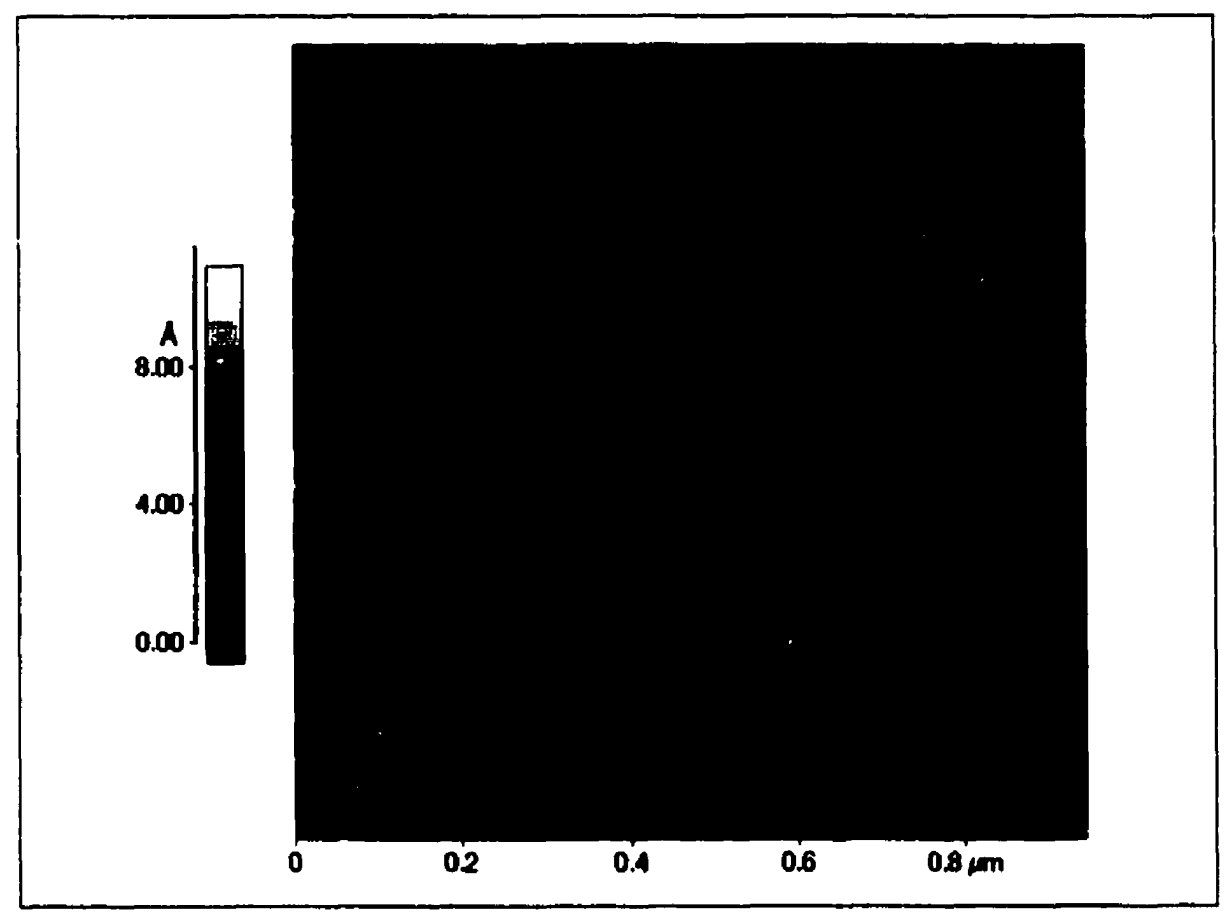

Figure i.13: AFM topography image for a GaAs reference buffer laver.

multitransient measurements.

Time-resolved energy spectra were constructed by recording the transient RDS signal at a single energy for individual periods of the SL growth [101]. Measurements were made from $1.5 \mathrm{eV}$ to $4.9 \mathrm{eV}$ in increments of $0.2 \mathrm{eV}^{\prime}$ with each transient synchronized to the same instant of the exposure cycle. These data were then reorganized to form 400 18-point spectra at $0.125 \mathrm{sec}$ increments. After these 18 cycles, the transient signal at $2.6 \mathrm{eV}$ was monitored during the growth of $\tau$ additional SL periods to ensure the reproducibility of the first 10 cycles, without oscillations.

For these SLs, with total thicknesses of $\sim 3600 \mathrm{~A}$, the accumulated strain caused by the lattice mismatch of $\operatorname{InAs}$ and $\mathrm{GaAs}(\sim 7 \%)$ results in misfit dislocation formation in the films. This effect manifests itself as a cross-hatched surface morphology observed using AFM. Notwithstanding, the RDS spectra, characteristic of the local 


\begin{tabular}{|c|c|}
\hline \hline Temperature $\left({ }^{\circ} \mathrm{C}\right)$ & Growth Rate $(\mathrm{A} / \mathrm{sec})$ \\
\hline 450 & $6.88 \pm 0.06$ \\
\hline 500 & $7.28 \pm 0.06$ \\
\hline 550 & $7.56 \pm 0.06$ \\
\hline 600 & $i .44 \pm 0.06$ \\
\hline \hline
\end{tabular}

Taijle i.t: NOVPE growth rales used in SL sample preparation for in-situ RDS study.

dimer arrangements, were unaffected by this long-range effect. AFM images of the SL structures are presented in Figure $\mathbf{i} .14$. Note the very straight, well-defined lines in the cross-hatching and the perfect $90^{\circ}$ intersections.

Figure 7.15 shows RDS energy spectra at several temperatures. obtained for the GaAs surface held under TBAs, and during GaAs growth. All data presented here show the real part of the RDS.

$$
\frac{\Delta r}{r}=2 R \epsilon\left[\frac{\dot{r}_{110}-\dot{r}_{110}}{\dot{r}_{110}+\dot{r}_{110}}\right] \text {. }
$$

which is primarily related to the imaginary part of the dielectric function [96]. These data may be interpreted using the "RDS database" described in Section 5.3 [97]. At all temperatures the GaAs surface under TBAs corresponds to the $\mathrm{d}(4 \times 4)$ reconstruction. distinguished by the negative feature near $2.6 \mathrm{eV}$ due to As dimers along the [110]axis [99]. and the peak near $4.0 \mathrm{eV}$. Two or more outer layers of As terminate this surface. While the spectra under TBAs are temperature-independent, this is not the case for the GaAs growing surfaces. At $400{ }^{\circ} \mathrm{C}$ the spectrum exhibits weakly positive features near $2.6 \mathrm{eV}$ and $4.2 \mathrm{eV}$, and a negative dip near $2.1 \mathrm{eV}$. This indicates the presence of both As and Ga dimers on the surface, presumably due to incomplete cracking of TBAs at lower temperatures. The spectrum may be associated with a (1×6)-like reconstruction [98]. With increasing $T_{g}$ the spectra show a trend towards the $\mathrm{d}(4 \times 4)$-like signature. At $600{ }^{\circ} \mathrm{C}$ the growing surface is indistinguishable from the surface held under TBAs.

A series of samples was grown, each consisting of one SL period, to study the $T_{g}$-dependence of the 2.6 eV RDS transient response as shown in Figure 7.16. The principle illustrated in Figure 7.15 is similarly evident in these data: the RDS signal 
a)

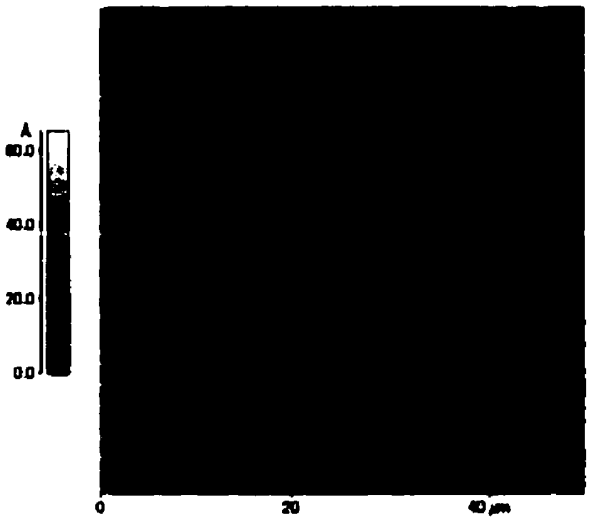

c)

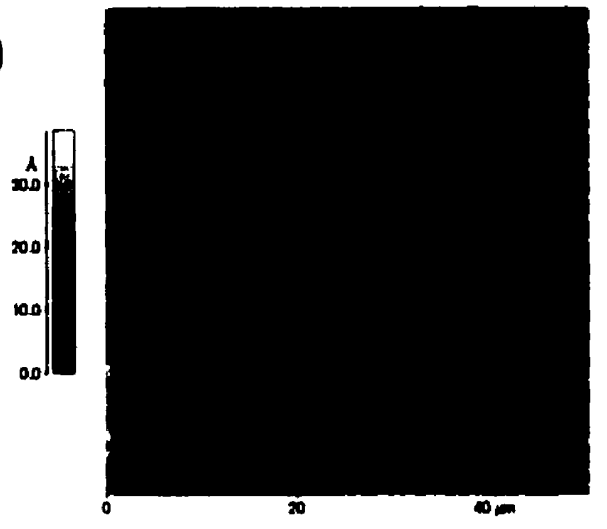

b)

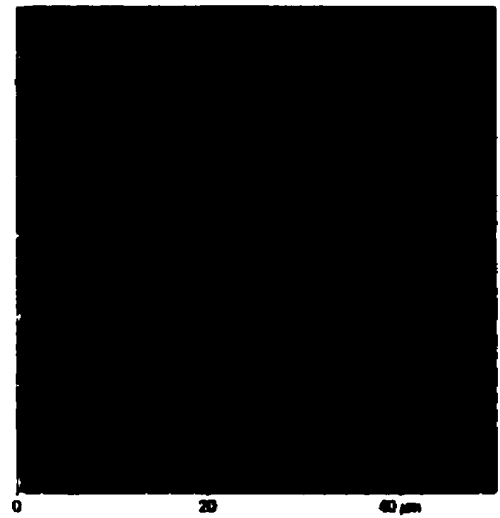

d)

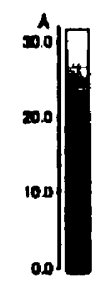

Figure 7.14: AFM topography images for SL structures described in the text. The samples were prepared at temperatures: a) $450^{\circ} \mathrm{C}$. b) $500^{\circ} \mathrm{C}$, c) $550^{\circ} \mathrm{C}$ and d) $600^{\circ} \mathrm{C}$.

shows a distinct change when the GaAs growth is initiated, an effect which is reduced at higher $T_{g}$. When the TEGa flow is discontinued and the surface is held under TBAs, the signal decays back to the TBAs-surface level, with a rate constant exhibiting a strong $T_{g}$-dependence. For the sample at $400{ }^{\circ} \mathrm{C}$ the signal does not completely recover during the $20 \mathrm{sec}$ TBAs pause, while the transient for the $600{ }^{\circ} \mathrm{C}$ sample drops to its original level almost instantly. This behavior is likely related to the TBAs molecular decomposition, which is complete above about $475{ }^{\circ} \mathrm{C}$. The decay time is thus representative of the time required to form an excess layer of As on the 


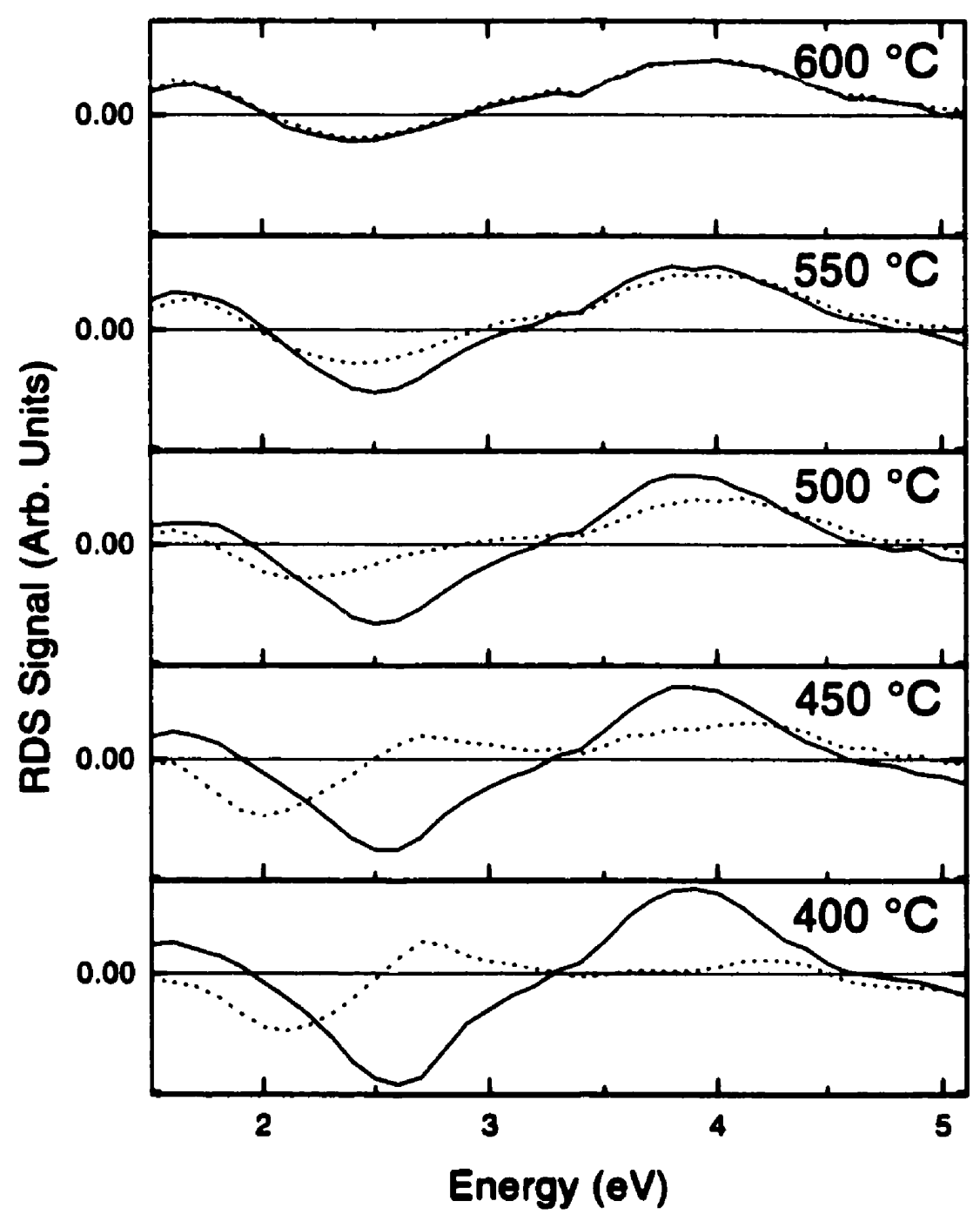

Figure 7.15: Temperature dependence of the RDS signal $(\Delta r / r)$ for GaAs surfaces under TBAs (solid lines) and during GaAs growth (dashed lines). The spectra were corrected for zero offsets using spectra obtained with the susceptor rotated $90^{\circ}$. 
surface.

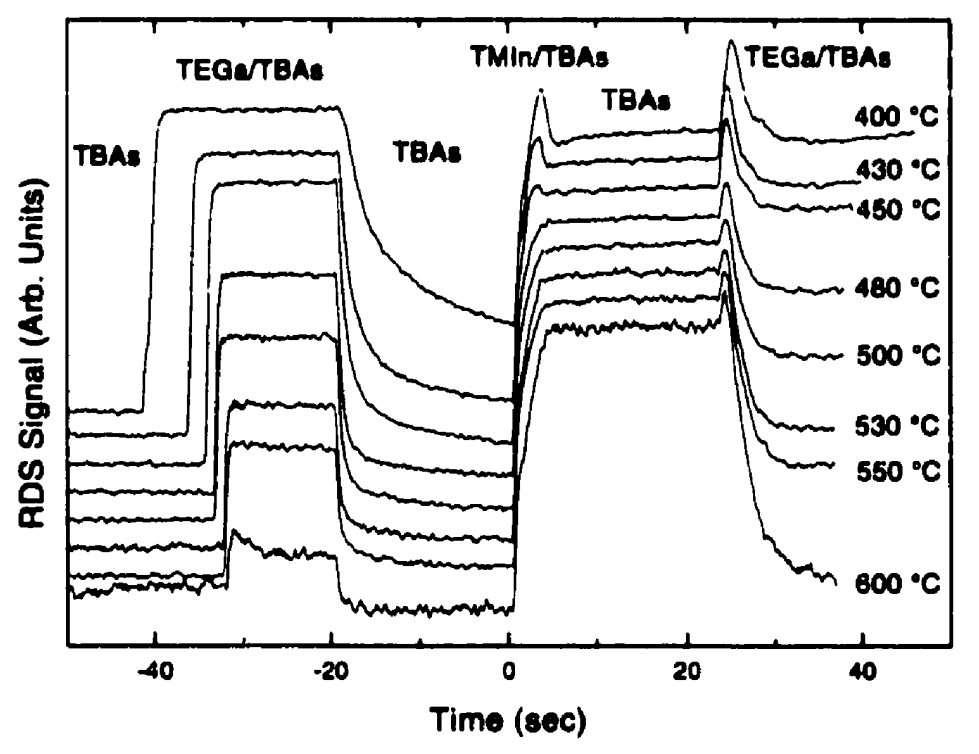

Figure $7.16: 2.6 \mathrm{eV}$ RDS transients during single SL cycles, with temperatures as noted. The transients were normalized and offset by arbitrary amounts to permit clear comparisons. The zero of time corresponds to the onset of the TMlin flow.

At all temperatures the RDS signal increases abruptly when the TMIn flow begins. The signal exhibits a "peak overshoot" at the lowest temperatures, where the signal increases steadily to its maximum value and then decreases gradually until the TMIn flow is terminated. This effect becomes less pronounced at higher temperatures and is no longer evident above $450{ }^{\circ} \mathrm{C}$. This behavior is similar to that reported for heteroepitaxy of ternary and quaternary materials [105].

Following a $20 \mathrm{sec}$ TBAs purge of the InAs-on-GaAs surface. which stabilizes the signal level, the RDS signal at all temperatures increases abruptly when the TEGa flow is initiated, rises to a maximum and then decays. In this case the transient peak is observed at all temperatures. Using similar samples with much thicker GaAs cap growths we have established that this signal actually exhibits a weak, damped 
oscillation behavior [105] with a period of the order of 1 minute. This effect is not apparent in the short $100 \AA$ GaAs growth for the samples of Figure 7.16 .

Figure 7.17 shows the $2.6 \mathrm{eV}$ RDS transient recorded during the growth of the first $10 \mathrm{SL}$ periods at $500{ }^{\circ} \mathrm{C}$. Consecutive cycles are quite reproducible and exhibit the features observed in Figure 7.16. In addition. the series of cycles follows a very slight damped oscillatory envelope which dies out during these 10 cycles. Subsequent cycles are highly reproducible with no oscillations.

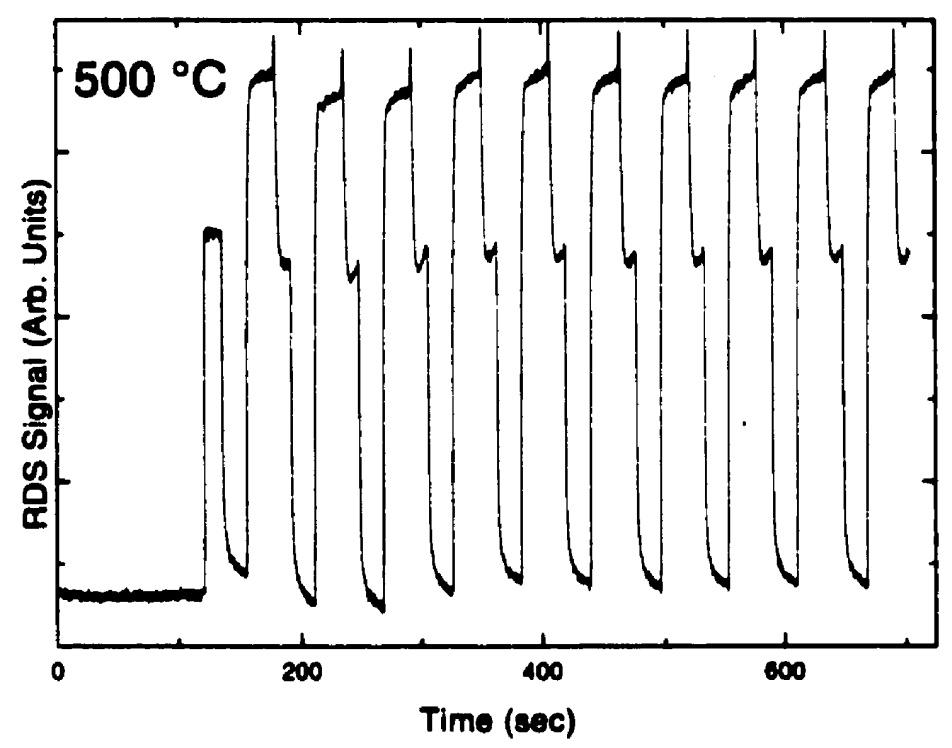

Figure $7.17: 2.6 \mathrm{eV}$ RDS transients for the first 10 periods of the $\mathrm{SL}$ growth at $500^{\circ} \mathrm{C}$. These cycles allowed the signal to stabilize before the acquisition of the multitransient spectra.

After these 10 stabilization cycles the full time-resolved RDS energy spectra were collected. Figures $\bar{\tau} .18$ and $\bar{\tau} .19$ each contain a series of such spectra corresponding to different stages of the growth of a single SL period, at growth temperatures of $450{ }^{\circ} \mathrm{C}$ and $600{ }^{\circ} \mathrm{C}$, respectively. For the sample at $450^{\circ} \mathrm{C}$ the first spectrum (Figure $\bar{i} .18(\mathrm{a})$ ) depicts the GaAs surface under TBAs similar to Figure 7.15 . When the InAs growth is 


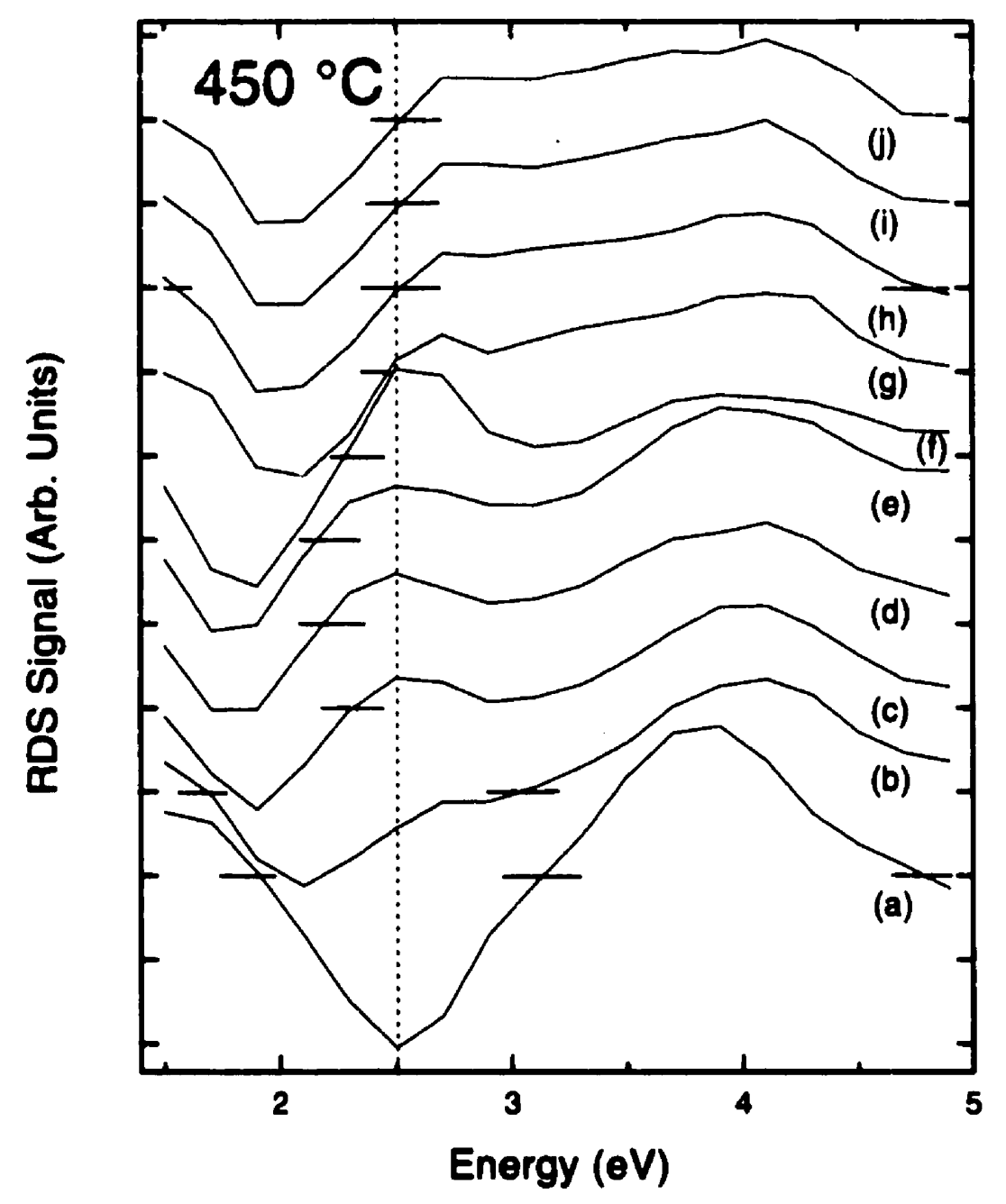

Figure 7.18: Multitransient energy spectra representing the development of surface anisotropies for the growth of a single SL period at $450^{\circ} \mathrm{C}$. These spectra correspond to (a) GaAs after 20 sec TBAs pause; (b)-(d) 1, 2 and 3 sec of InAs growth; (e) after 20 sec TBAs pause; (f)-(j) $1,3,5,7$ and 9 sec of GaAs cap growth. Consecutive spectra were offset from one another for clarity. The short, horizontal lines indicate zero levels. 


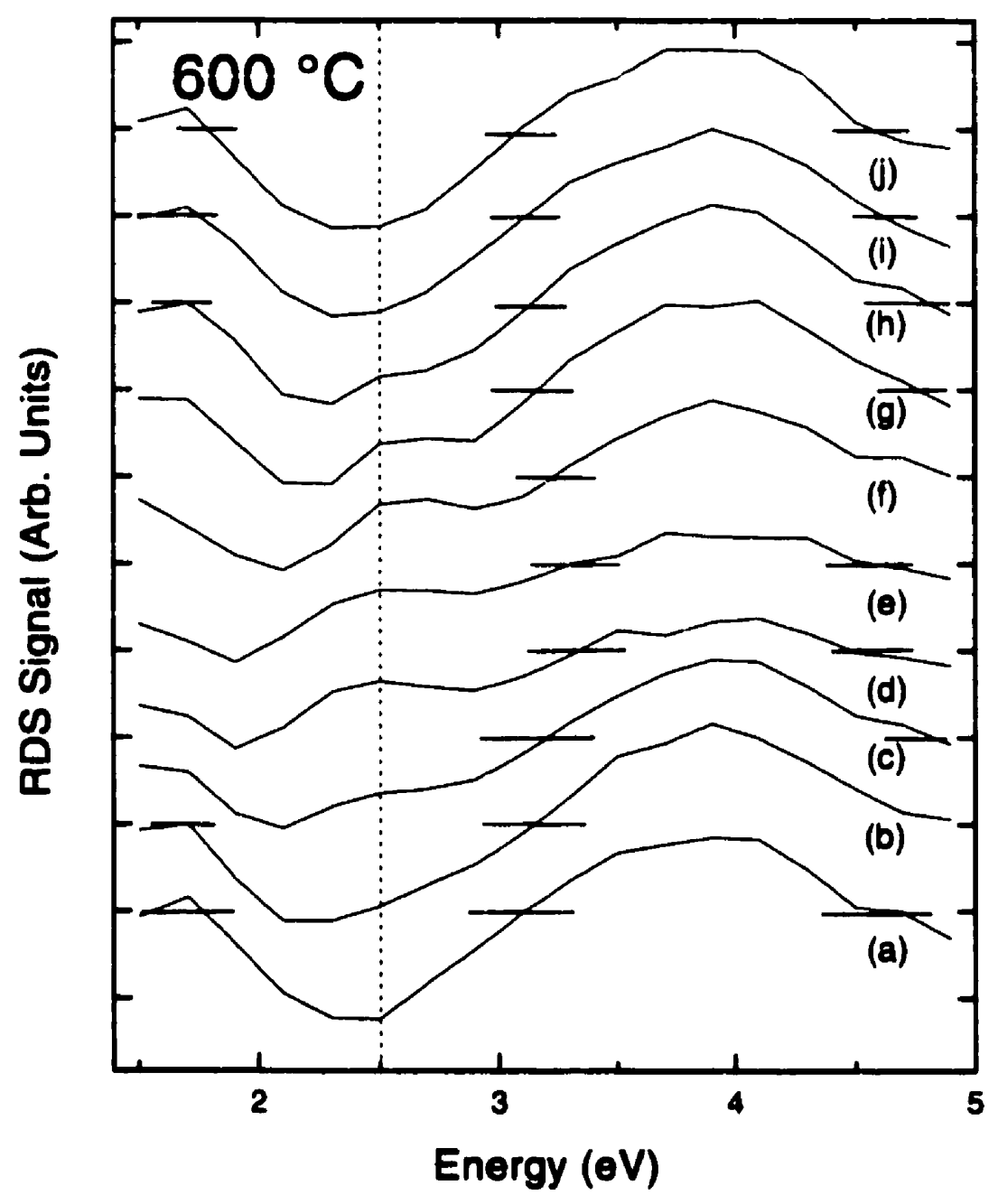

Figure 7.19: Multitransient energy spectra representing the development of surface anisotropies for the growth of a single SL period at $450{ }^{\circ} \mathrm{C}$. These spectra correspond to (a) GaAs after 20 sec TBAs pause; (b)-(d) 1, 2 and 3 sec of InAs growth; (e) after $20 \mathrm{sec}$ TBAs pause; (f)-(j) 1, 2, 3, 5, and $6 \mathrm{sec}$ of GaAs cap growth. Consecutive spectra were oftset from one another for clarity. The short, horizontal lines indicate zero levels. 
initiated the RDS minimum moves rapidly to near $1 . \bar{\imath} \mathrm{eV}$ (Figure $i .18(\mathrm{~b})-(\mathrm{d})$ ). The negative feature of the TBAs-ambient surface at $2.5 \mathrm{eV}$ becomes positive with the impinging TMIn molecules. A local minimum develops in the spectrum near $2.9 \mathrm{eV}$. while the maximum near $3.9 \mathrm{eV}$ remains similar to the feature observed for the TBAsambient surface. These spectral characteristics observed during InAs growth agree very well with those reported by Zorn et al. [10i] which were identified by low energy electron diffraction as arising from a $(2 \times 4)$ - like InAs reconstruction. The surface evolution during InAs growth has many similarities to that observed by Scholz et al. in MBE [1.37]. although it should be emphasized that our data are representative of the growing surface without interruptions.

The InAs-on-GaAs surface is essentially unchanged during the following $20 \mathrm{sec}$ under TBAs (Figure $\mathrm{T} .18(\mathrm{e})$ ). When the GaAs growth is initiated (Figure $\mathrm{T} .18(\mathrm{f})$ ) the 2.5) $\mathrm{el}$ feature increases very rapidly, accounting for the peak behaviour noted in Figure 7.16 . The origin of this strong anisotropy is not clear at present. Within approximately $3 \mathrm{sec}$ (Figure $i .18(\mathrm{~g})$ ) the spectrum reaches the steady-state characteristic of $450^{\circ} \mathrm{C}$ GaAs growth. the $(1 \times 6)$-like spectrum identified in Figure 7.15 . It is noteworthy that during InAs growth the RDS spectrum reaches a stable $(2 \times 1)$-like InAs spectrum during the growth of only $1 \mathrm{ML}$. but for GaAs cap growth the $3 \mathrm{sec}$ required for the signal to stabilize represents approximately $20 \mathrm{~A}(\overline{\mathrm{ML}})$ of material. This may be related to In segregation. as observed during ALE growth [90]. although the effect can not be clearly inferred from the data.

It is tempting to suppose that during the cap growth the total RDS spectrum, $(\Delta r / r)$, consists of a linear combination of the InAs. $(\Delta r / r)_{I n A s .}$ and GaAs. $(\Delta r / r)_{\text {GidAs. }}$. spectra. i.e. $(\Delta r / r)=y(\Delta r / r)_{I n A s}+(1-y)(\Delta r / r)_{G a A s}$, where $y$ is the fraction of In on the surface. Attempts to model the data in this way were prohibited by the strong peak overshoot occurring at the beginning of the cap layer growth.

At higher $T_{g}$ many of the preceeding features are also observed. One main difference. visible in Figure $\mathbf{7 . 1 9}$, is that the spectra obtained during the GaAs cap growth (Figure $\mathrm{i} .19(\mathrm{f})$-(j)) quickly recover to the $\mathrm{d}(4 \times 4)$-like lineshape. as opposed to the (l×6)-like GaAs spectrum observed at lower $T_{g}$. This is consistent with the spectrum 
for the GaAs growing surface shown in Figure 7.15 and is related to the higher effective As-partial pressure under these conditions. In addition. evidence of the In-related feature at $2.5 \mathrm{eV}$ persists for longer than $3 \mathrm{sec}$ following GaAs capping, corresponding to almost $8 \mathrm{ML}$ of growth.

XRD results from the SL structures are shown in Figure 7.20 . Comparing the experimental curves with RADS simulations (Section 3.3) for an ideal SL (35 periods of $1 \mathrm{ML}$ InAs/100 A GaAs). it is found that the SL satellite features are reasonably narrow. but slightly broader than for the ideal structure. This broadening is due to the presence of misfit dislocations in the crystal corresponding to the cross-liatched patterns in Figure 7.14.

\subsubsection{XSW Study of MOVPE-Grown Films}

The SL structures and the RDS characterization presented in the preceeding section were used to gain an understanding of the InAs/CiaAs heteroepitaxial growth mechanisms, and the $T_{y}$-dependence of these processes. The goal of this was to provide a basis for understanding the structural details of the buried InAs 1 .IL and 1/2 ML SQWs which will now be discussed. In the following the main experimental results of this thesis will be presented. The section will begin with a description of the sample preparation and will then present the results of XRD. XSW. XAFS and PL studies.

Epitaxy was performed at 50 Torr using SI VGF GaAs(001) epi-ready substrates with no additional cleaning, beginning with the growth of a standard buffer. Two series of samples were prepared for this study because of the conflicting requirenents of the XSW and PL measurements. In the XSW case. the GaAs cap thicknesses must be sufficiently small to prevent interference effects (Pendellösung) between the diffracted beams from the substrate and the cap. A thickness of about $100 \mathrm{~A}$ was appropriate for this. For PL measurements a thicker cap is required to prevent the reduction of the In-excitonic emission due to nonradiative recombination at the sample surface. $A$ thickness of about $2000 A$ is more than adequate for this purpose.

Following the buffer layer growth, each sample was allowed to equilibrate at the 


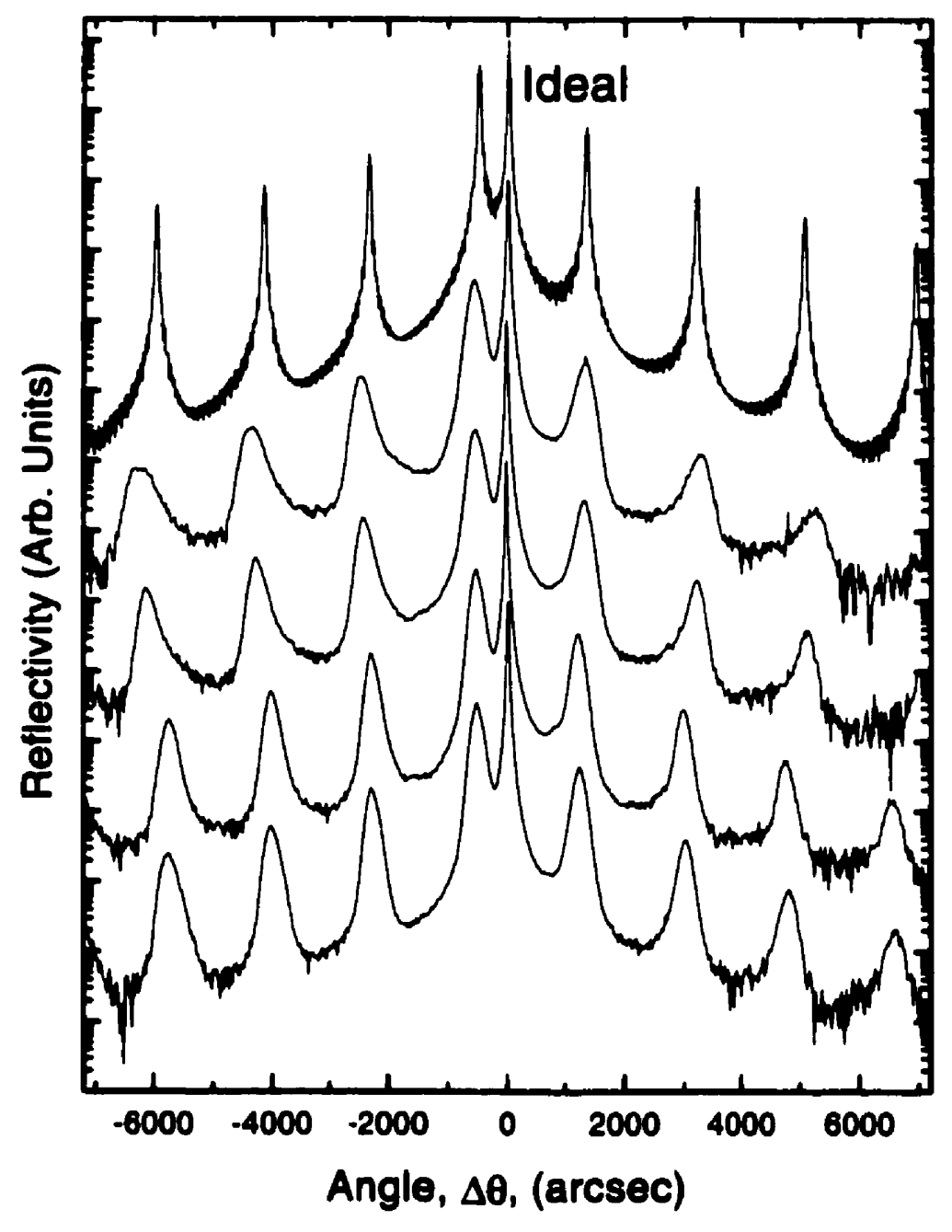

Figure 7.20: XRD results for the SL structures as a function of angle near the $\mathrm{GaAs}(004)$ reflection. Data for samples grown at $450{ }^{\circ} \mathrm{C}, 500{ }^{\circ} \mathrm{C}, 550{ }^{\circ} \mathrm{C}$ and $600{ }^{\circ} \mathrm{C}$ are shown in ascending order and are offset by two decades for clarity. The top curve shows the simulation results for an ideal SL structure, as indicated. 
desired growth temperature. $T_{g}$. GaAs barrier layers of thickness $\sim 100 \AA$ were deposited above and below the In.As laver ( $1 \mathrm{ML}$ or $1 / 2 \mathrm{ML}$ ) which was also grown at $T_{g}$ using TMIn at $1.98 \mu \mathrm{mole} / \mathrm{min}$ and TBAs at $198 \mu \mathrm{mole} / \mathrm{min}$. yielding a growth rate of approximately I ML per $3 \mathrm{~s}$. For the PL samples an additional GaAs cap laver $(\sim 1900 \mathrm{~A})$ was deposited at $580^{\circ} \mathrm{C}$.

\subsubsection{X-Ray Diffraction}

HRXRD data for $1 \mathrm{ML}$ and 1/2 ML samples are shown in Figures 7.21 and 7.22 . The experimental curves are compared to RADS simulations using a 3-layer model consisting of the GaAs substrate, a single In-containing layer, and a GaAs cap layer. For the I ML samples. the thickness of the strained InAs layers used in the simulations was $3.25 \mathrm{~A}$. as predicted by the MET [52]. assuming single layers of In atoms and coherency of the InAs layers with the GaAs substrate. For the 1/2 ML samples the MET was also used. assuming single $\ln _{r} \mathrm{C} a_{1-r}$.ts lavers. as will be discussed. From the interference fringes an accurate measurement of the Ciats cap thickness is obtained. However. this technique is slightly less sensitive to the distribution of In atoms in the SQW layer, particularly in the case of these sandwich structures with very thin GaAs caps. It was pointed out [1] that complementary information may be obtained by using XSW in addition to XRD.

\subsubsection{XSW Analysis}

Figures 7.23 and 7.24 show the XSW data for the 1 and $1 / 2$ ML InAs samples prepared at several growth temperatures. MET [52] predicts that a single InAs ML in GaAs has $P^{H}=1.146$ under pseudomorphic conditions [2]. Comparing the raw data of Figures 7.23 and 7.24 with Figure 7.1 it is clear that the In atoms do not occupy the same positions as the substrate $\mathrm{Ga}$ atoms. The best I ML sample, structurally, is the one with $T_{g}=5,50^{\circ} \mathrm{C}$, having an ideal $P^{H}$ and the highest $F^{H} .1 / 2 \mathrm{ML}$ samples grown at 400 and $600^{\circ} \mathrm{C}$ have similar $P^{H}$-values to one another and high values of $F^{H}$. Notice the more bulk-like characteristics of the 1/2 ML data, specifically the pronounced dip at the low-energy side of the Bragg reflection. Assuming that the 


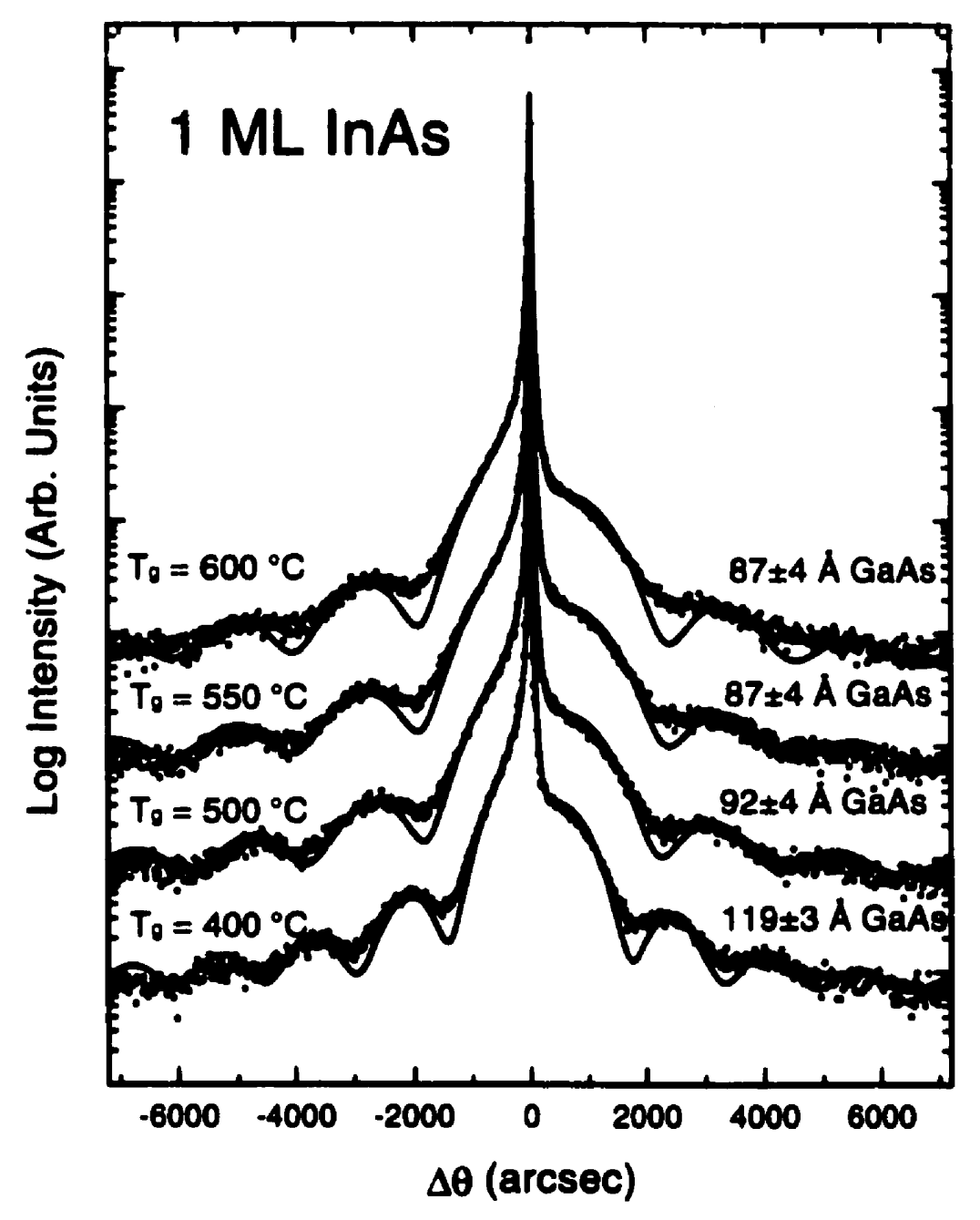

Figure 7.21: HRXRD results obtained near the GaAs(004) symmetric substrate reflection. The data for the $1 \mathrm{ML}$ samples with several growth temperatures are indicated by points. Solid lines denote the results of dynamical simulations with the Ga.As cap thicknesses indicated, as described in the text. The curves were offset for clarity. 


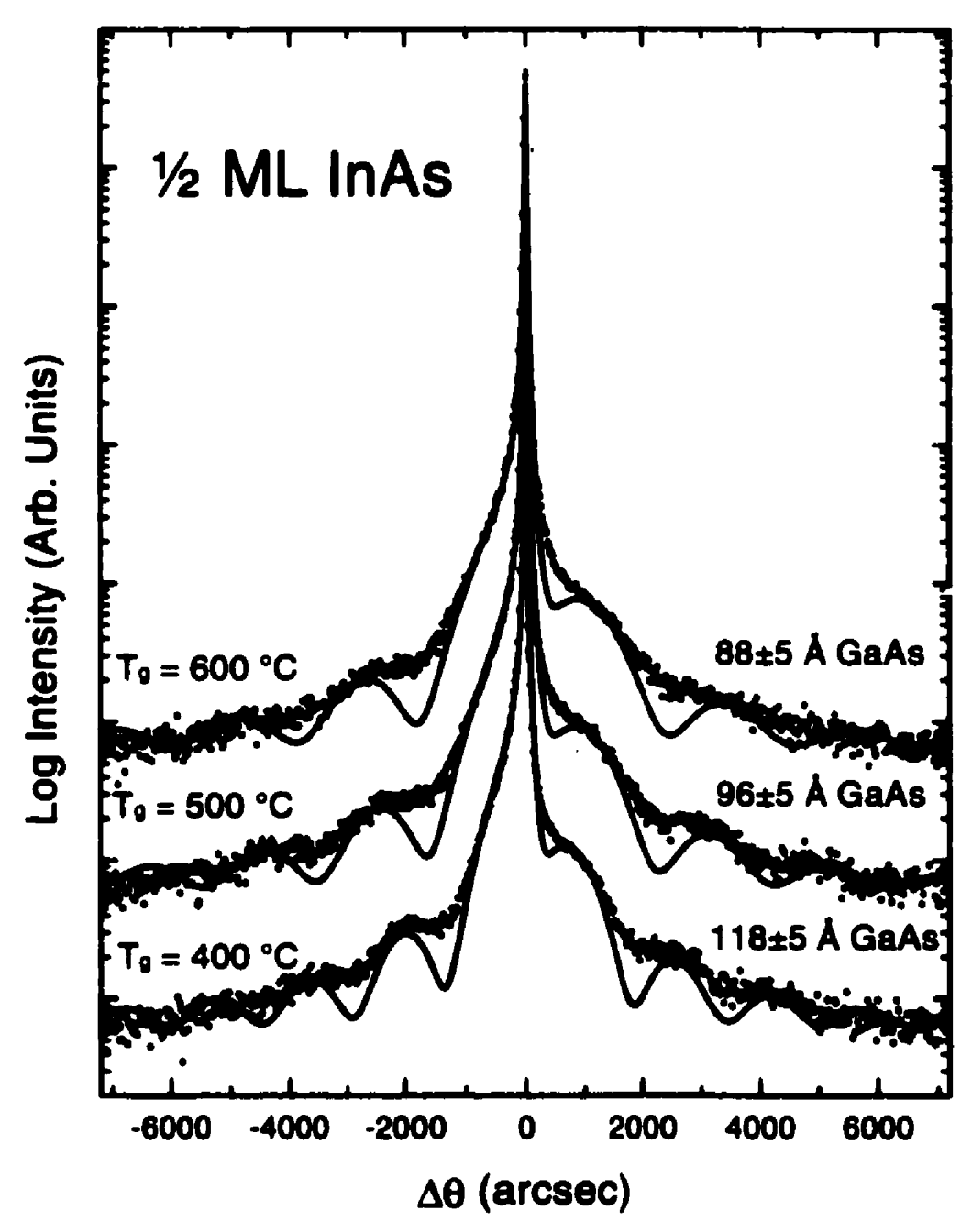

Figure 7.22: HRXRD results obtained near the GaAs(004) symmetric substrate reflection. The data for the 1/2 ML samples with several growth temperatures are indicated by points. Solid lines denote the results of dynamical simulations with the GaAs cap thicknesses indicated, as described in the text. The curves were offset for clarity. 
1/2 MLs consist of single $\ln _{x} \mathrm{Ga}_{1-r}$ As layers and using the method of Section 7.2 the following values of $x$ were found from the XSW results: $0.63 \pm 0.14,0.56 \pm 0.14$ and $0.69 \pm 0.14$, in order of increasing $T_{g}$.

To clarify the mechanism producing the higher-than-ideal coherent positions in the $1 \mathrm{ML}$ samples with the lowest $T_{g}$-values, we consider the possibility of In atoms occupying multiple sites in the crystal. For In atoms occupying $n$ positions $\left(P_{i}^{H}\right)$ relative to the diffraction planes. Equations 3.54 and 3.55 give [32]

$$
F^{H}=\left(G_{H}^{\prime}{ }^{2}+C_{H}^{s}\right)^{1 / 2} .
$$

and

$$
P^{H}=\frac{1}{2 \pi} \tan ^{-1}\left(\frac{G_{H}^{s}}{G_{H}^{c}}\right)+\left\{\begin{array}{cc}
0.5 & \text { if } G_{H}^{i}<0 \\
0 & \text { otherwise }
\end{array} .\right.
$$

with $C_{H}^{c}$ and $C_{H}^{\text {s }}$ defined in Section 3.2.2

Previous XSW measurements [1] were interpreted assuming In surface segregation during the GaAs cap layer growth. ('onsider the possible results of an XSW measurement for a buried layer in $\operatorname{GaAs}(001)$ including this process, with I ML total In content. It is very important to note that a single XSW measurement provides only one Fourier component of the In distribution. This presents a uniqueness problem when one attempts to infer additional information in this way, although the measured values do place some constraints on the structural possibilities.

The issue of In segregation was discussed in Section 5.2. We consider two possibilities: (i) Each group III layer consists of an In. (ia alloy. with the composition of sequential layers graded in the growth direction. The composition of the $n$th laver. $\operatorname{In}_{x_{n}} \mathrm{Ga}_{1-x_{n}}$ As, is $x_{n}=(1-\sigma) \sigma^{n-1}$ from Equation 5.7 and the strained lattice constant of each layer is found from Equations $7.8-7.11$. In this scenario the In atoms are located at $1 / 2$ the lattice constant of the strained layer, which is a function of $x_{n}$. The other possibility is: (ii) Each group III layer contains In and Ga atoms as above, with the In atoms arranged in equidistant "terraces" or islands. For each layer the tetragonally distorted InAs cell is expanded $14.6 \%$ in the (001)-direction compared to GaAs. This is the scheme used in Ref [1]. The two models are illustrated in Figure 7.25. 


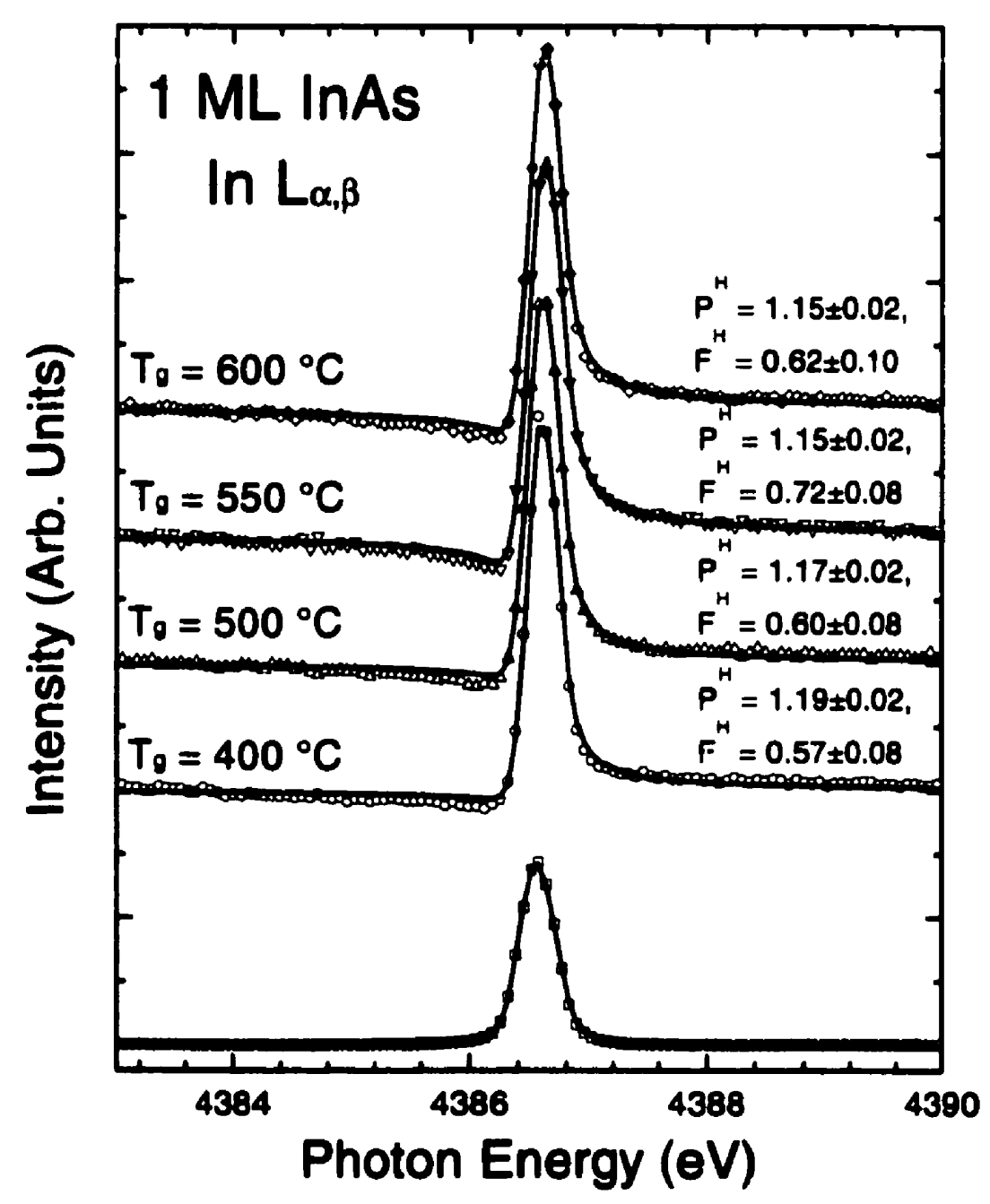

Figure 7.23: Photon energy dependence of the reflectivity (bottom curve) and the In$\mathrm{L}$ fluorescence yield near the $\mathrm{GaAs}(004)$ Bragg backreflection condition for a series of 1 ML InAs samples grown by MOVPE, with growth temperatures as indicated. The solid lines are the best fits to the data points and the curves were offset for clarity. 


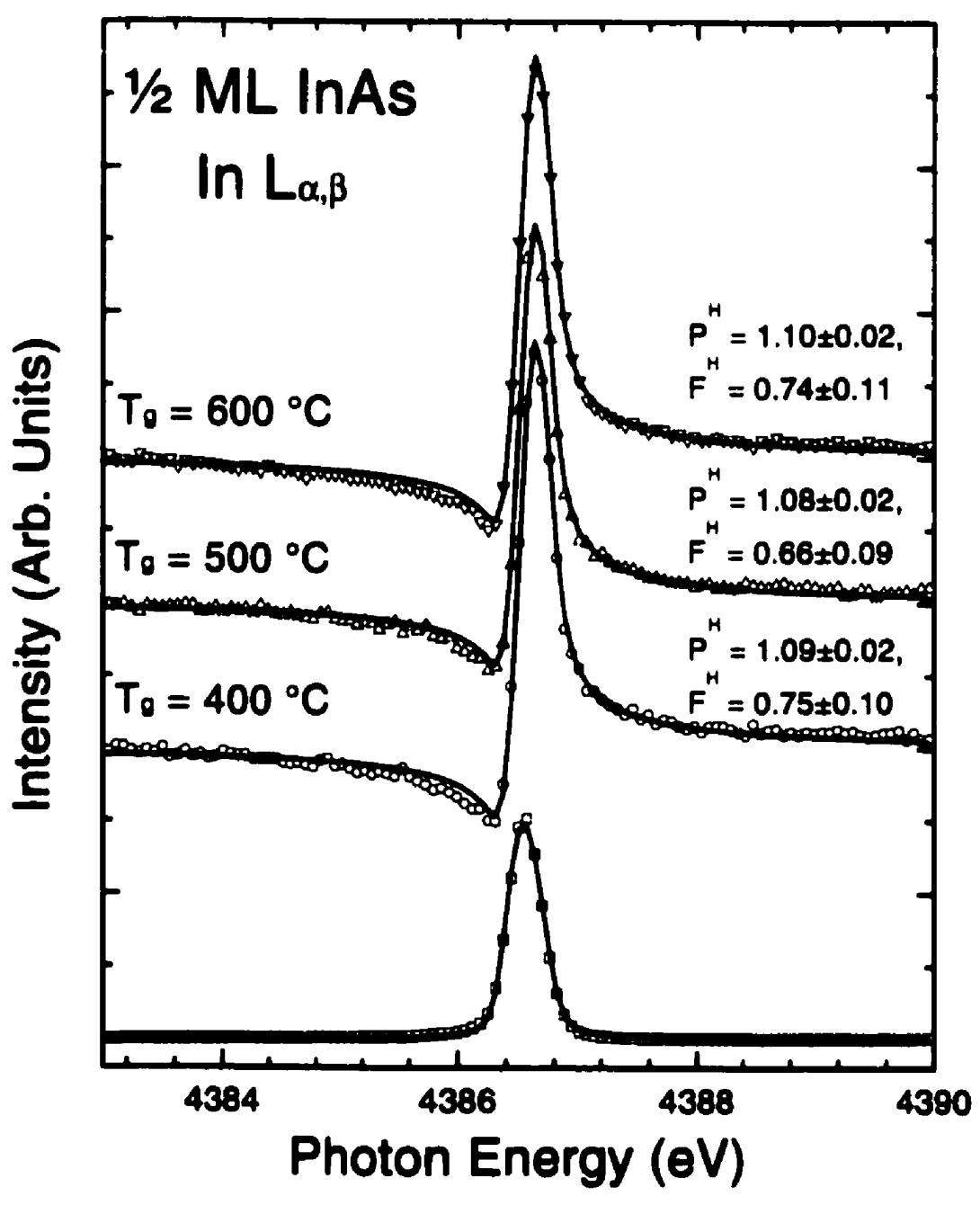

Figure 7.24: Photon energy dependence of the reflectivity (bottom curve) and the In-L fluorescence yield near the GaAs(004) Bragg backreflection condition for a series of $1 / 2$ ML InAs samples grown by MOVPE, with growth temperatures as indicated. The solid lines are the best fits to the data points and the curves were offset for clarity. 
It should be noted that (i) implicitly uses the VCA by assuming that both In and $\mathrm{Ga}$ atoms reside in the same cation plane. Mikkelsen and Boyce's study proved that the In-As and Ga-As bond lengths in the ternary alloy do not deviate significantly from their native values [54], and that the atoms do not occupy the VCA positions. Model (i) should be considered as a first approximation in lieu of a more complete description.

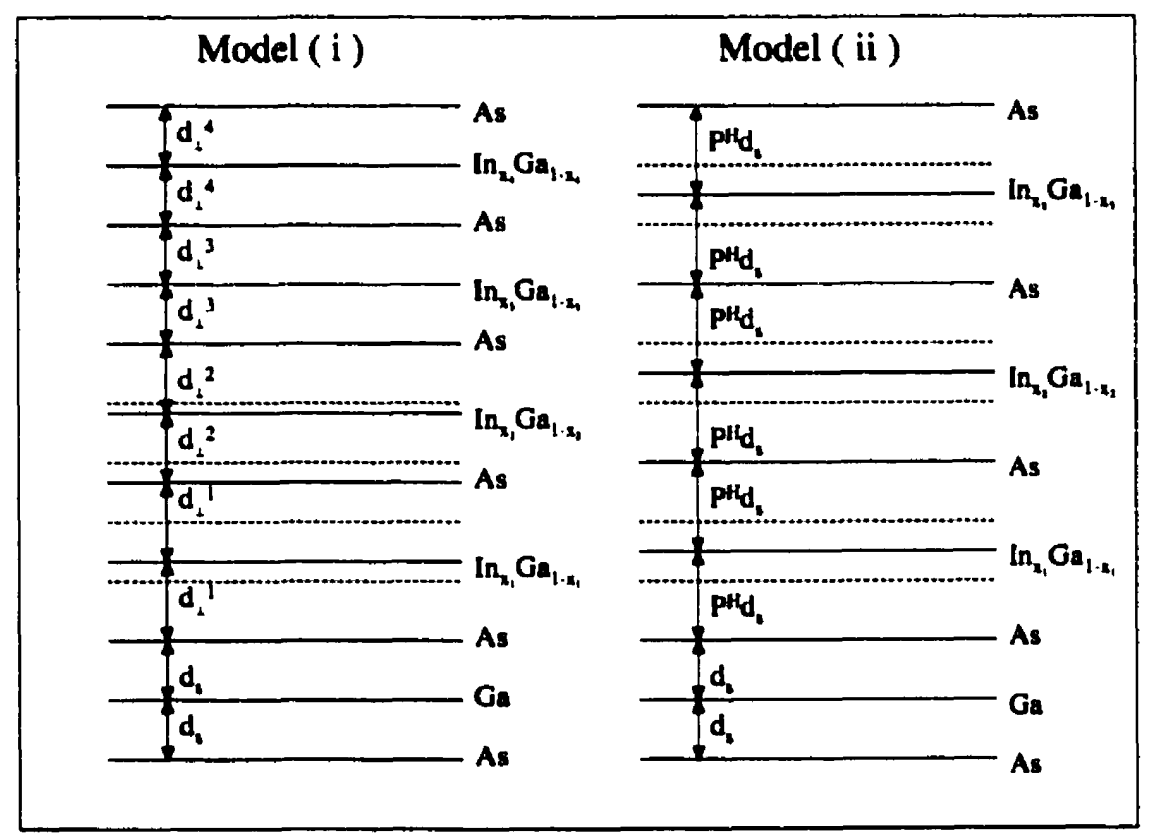

Figure 7.25: The distribution of In atoms relative to the GaAs(004) planes according to the two models described in the text. In model (i) the distance. $d_{\perp}^{i}$, between each cation plane and its corresponding As plane is a function of the In concentration. $x_{n}$. For model (ii) the spacing is the same for all planes. independent of In content.

Model (ii) assumes terracing of the In atoms, and neglects edge effects which are likely present at the boundaries between the InAs and the surrounding GaAs. Additionally, this assumes equal strain in all InAs layers in spite of the differences in In content. In this respect model (i) is somewhat more realistic. 
The possible XSW parameters have been estimated using both models. In case (i), with a given $\sigma$, the lattice constant for the $n$th layer was estimated using Vegard's law and Equation 5. $i$ to give the bulk lattice constant. The effect of strain was included using MET as discussed previously. If the (004)-plane spacing between the $i$ th In.Ga layer and the corresponding As-plane is denoted by $d_{\perp}^{i}$, then the total distance of the $n$th layer from the last As-plane of the substrate is

$$
d^{n}=2 \sum_{i=1}^{n-1} d_{\perp}^{i}+d_{\perp}^{n}
$$

These positions may then be substituted into Equations 3.56 and 3.57 from which $F^{H}$ and $P^{H}$ are determined.

The results for a range of segregation coefficients are shown in Figures $\overline{7} .26$ and $\bar{\tau}: 2 \overline{7}$ for the two cases. For model (i) in which the segregation produces a graded InCia.ts alloy, the theoretical XSW parameters $P^{H}$ and $F^{H}$ are found to decrease with increasing segregation. bit remain close to the values for the ideal ML. It is clear from the figures that only (ii) can explain the experimental results in which $P^{H}>1.15$. For samples grown at $400^{\circ} \mathrm{C}$ and $500^{\circ} \mathrm{C}$. the XSW coherent positions are found to be consistent with $\sigma=0.30$. and $\sigma=0.15$. respectively, as indicated. Moreover, the experimental values of $F^{H}$ are consistent with this interpretation. if the Debve-Waller factor is included. Let us reemphasize that this interpretation of the . $\mathrm{XSW}^{2}$ data is not entirely conclusive.

Comparing the data with the island model, the segregation coefficients inferred from the data are found to be greater for the samples grown at the lowest temperatures. Since Giannini et al. measured only one sample using XSW [1]. the $T_{g}$-dependence was not discussed. They found $P^{H}=1.1 \imath \pm 0.02, F^{H}=0.58 \pm 0.0 i$ which was related to a segregation coefficient of $\sigma \approx 0.25$ when the structure was grown by MBE with the flashoff technique. In their experiment, the InAs ML and the first $5 \mathrm{ML}$ of the GaAs cap were deposited at $420{ }^{\circ} \mathrm{C}$. The temperature was then raised to $540^{\circ} \mathrm{C}$ to desorb the segregated In. Finally. $300 \AA$ of GaAs were deposited at $540{ }^{\circ} \mathrm{C}$.

Previous reports of In segregation in $\mathrm{GaAs}(001) / \mathrm{InAs} / \mathrm{GaAs}$ structures have used both thermodynamic and kinetic models to describe the process (Section 5.2). Several experiments [80]-[84] have found that In segregation increases with increasing 


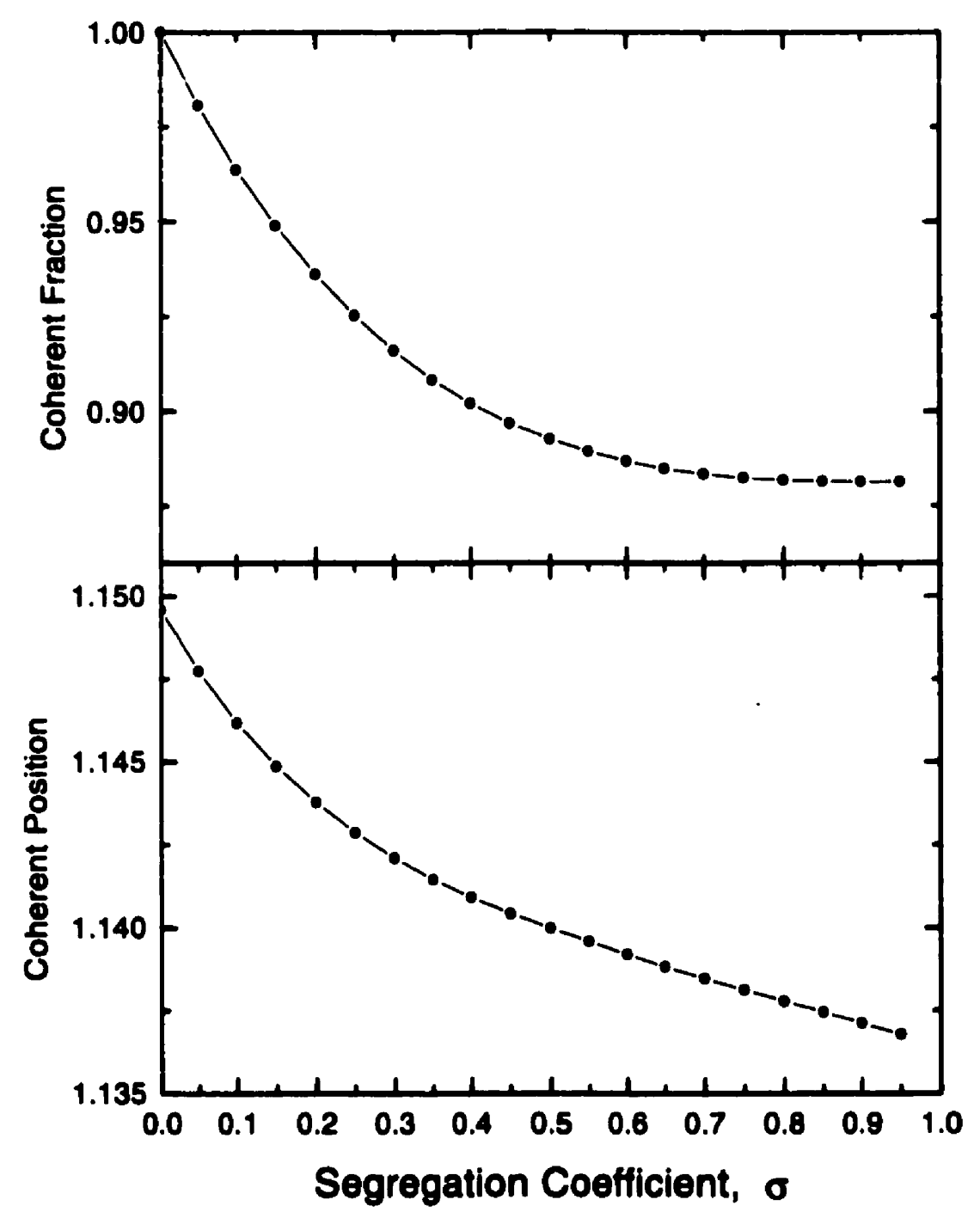

Figure 7.26: XSW parameters as a function of segregation coefficient, $\sigma$, predicted by model (i) described in the text, with $1 \mathrm{ML}$ total In coverage. 


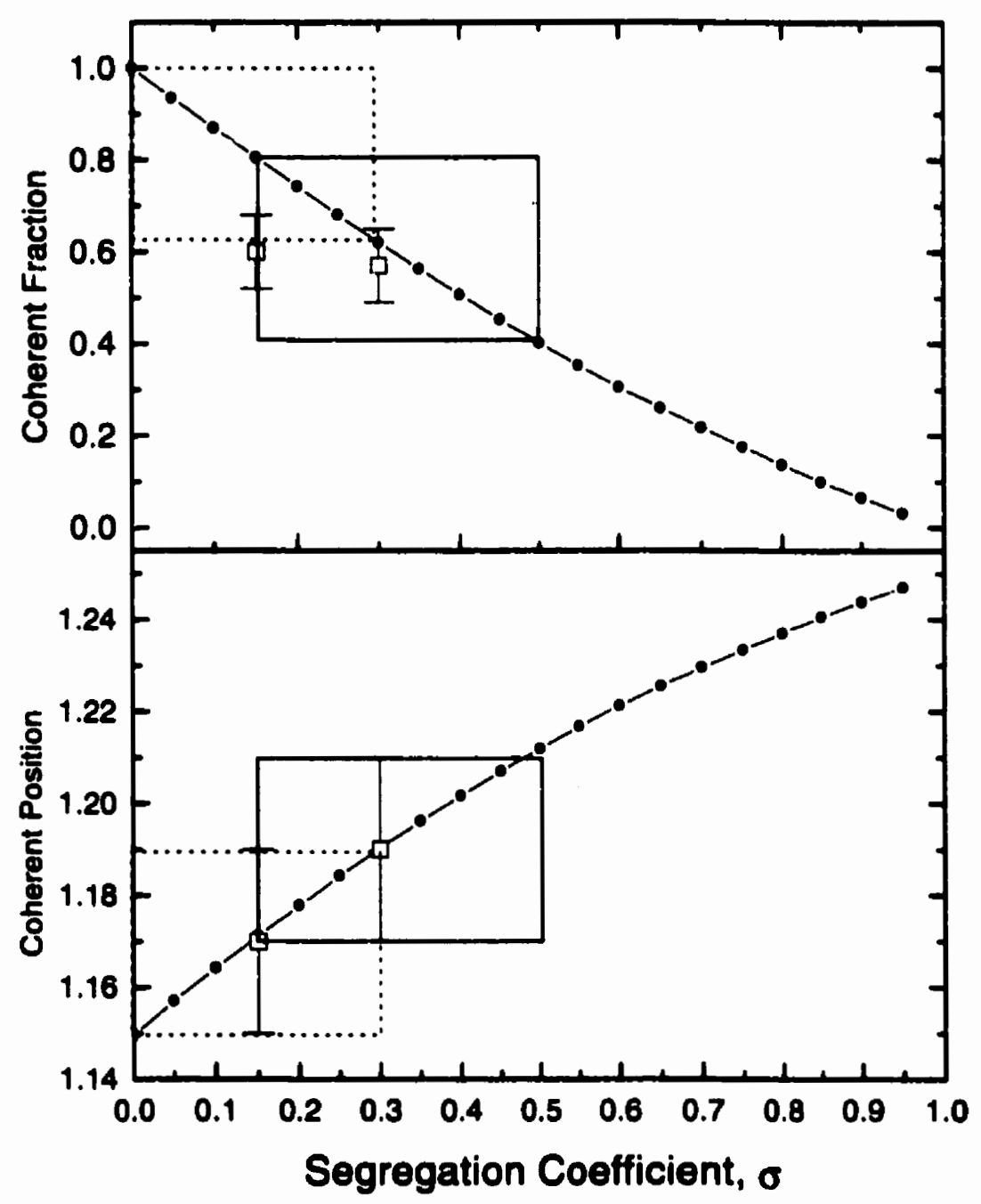

Figure 7.27: XSW parameters as a function of segregation coefficient, $\sigma$, predicted by model (ii) described in the text. In the bottom half of the figure the experimental XSW coherent positions (small hollow squares) are compared with the values of the segregation coefficient, $\sigma$, which might produce the measured $P^{H}$. The solid and dashed rectangles are used to indicate the range of possible values of $\sigma$ for the 1 ML samples grown at 400 and $500{ }^{\circ} \mathrm{C}$. respectively. In the top half of the figure the corresponding values of $F^{H}$ are indicated, as well as the experimental values. 
$T_{g}$, ruling out the thermodynamic model. The kinetic model [8.5] reproduces the fixed-temperature results and accounts for the observed increase with growth temperature [92]. The incident As flux has also been shown to affect the segregation [93]. and might be included in an even more complete model.

An interpretion of the $\mathrm{XSW}$ data using model (ii) would indicate increasing In segregation with decreasing $T_{g}$. This disagrees with the experimental evidence for MBE growth obtained by other means [80]-[84] due to differences in the growth conditions between MBE and MOVPE. The results for MBE-grown samples presented in Section 7.2 indicated that the growth temperature of the GaAs barriers also plays a pivotal role.

For the $1 \mathrm{ML}$ samples grown at $550^{\circ} \mathrm{C}^{\prime}$ and $600^{\circ} \mathrm{C}$ the $\mathrm{XSW}$ results indicate near ideal In distributions, particularly in the former. This is attributed to the favourable conditions created in the MOVPE environment [ 79$]$. Consider the requirements for minimal segregation suggested by other authors.

\begin{tabular}{|c|c|}
\hline \hline Temperature $\left({ }^{\circ} \mathrm{C}\right)$ & Growth Rate $(\mu \mathrm{m} / \mathrm{hr})$ \\
\hline 400 & $1.94 \pm 0.07$ \\
\hline 500 & $2.42 \pm 0.12$ \\
\hline 550 & $2.41 \pm 0.11$ \\
\hline 600 & $2.42 \pm 0.13$ \\
\hline \hline
\end{tabular}

Table 7.5: Temperature dependence of GaAs growth rate. from the GaAs cap thicknesses determined by XRD.

As shown in Table 7.5 , the GaAs cap growth rate is independent of temperature for $500{ }^{\circ} \mathrm{C} \leq T_{g} \leq 600{ }^{\circ} \mathrm{C}$. This is indicative of mass-transport-limited growth (Figure 2.4). Gérard et al. [it] suggested that the segregation process may be minimized by suitable kinetic adjustments during the cap layer growth. In the mass-transportlimited regime the growth may be less susceptible to a kinetic rearrangement of group III atoms, allowing a reduction in the In-Ga exchange reaction. At $400{ }^{\circ} \mathrm{C}$. which is in the surface-kinetic-limited regime. the growth kinetics may play a larger role. A strong $T_{g}$-dependence in the surface reconstruction during growth was shown in Figure 7.15 . This results from the incomplete decomposition of the TBAs molecules 
at lower $T_{g}$ which may contribute to the apparent segregation enhancement under these conditions.

Guille et al. $[8]$ suggested that very high GaAs growth rates could beat the In-Cia exchange rate. although this was not the case for up to $0.5 \mu \mathrm{m} / \mathrm{hr}$. Recent results [9:3] for MBE-grown InGaAs/GaAs structures at $5.1 \mu \mathrm{m} / \mathrm{hr}$ and $500{ }^{\circ} \mathrm{C}$ have noted a reduction in segregation due to the rapid surface coverage. At our higher temperatures the GaAs growth rate of $2.4 \mu \mathrm{m} / \mathrm{hr}$ may be sufficient to discourage segregation. At lower temperatures the reduced GaAs growth rate allows the segregation process to occur more prominently.

The time-resolved RDS study described in Section 7.6 .1 did not indicate any pronounced segregation during GaAs overgrowth. In contrast. clear evidence of In segregation was observed during ALE growth (Section $\bar{i} . t$ ) which has a much higher cation surface mobility than conventional MOVPE. This mobility produces the smooth interfaces which are associated with the technique. Unfortunately, the lack of As overpressure leads to enhanced exchange reactions between incoming $\mathrm{Gia}$ atoms and the underlying In [9, 84]. While ALE produces interfaces that are highly uniform and smooth, they suffer from reduced abruptness. Section $\mathbf{i} . t$ showed that In segregation cannot be eliminated in the ALE growth for any usual growth temperature.

We conclude that the combination of growth in a mass-transport-limited regime, high growth rate. and low cation surface mobility aid in reducing segregation effects in the $1 \mathrm{ML}$ structures produced at the higher temperatures. For lower- $T_{g}$ samples, the NSW data are indicative of enhanced In segregation. This arises because of surface kinetics effects: lower As coverage on the growing surface, and the lower GaAs growth rate. Although these factors are a direct consequence of the reduced temperature, the temperature itself is not the important parameter per se.

\section{ALE Results (Revisited)}

It is worth reconsidering the XSW results for ALE-grown samples. The data were interpreted in terms of $\mathrm{In}$ atoms arranged in single $\operatorname{In}_{\boldsymbol{r}} \mathrm{Ga}_{1-x}$ As layers, with $x<1$. This interpretation was used because the experimental values of $P^{H}$ were found to 
be less than the ideal value. However, this neglected the In segregation directly observed using RDS. Model (i) described in this section predicts that the segregated In atoms are arranged in alloy layers. with the layer composition graded along the growth direction. This model does predict values of $P^{H}<1.15$, although the lowest value predicted by the model is $P^{H}=1.13 \mathrm{i}$, for $\sigma=1.0$. This value is still greater than the highest $P^{H}$ measured for the ALE samples. It is possible that the ALE samples contain less than one complete ML of incorporated In, as suggested previously, and that the In distribution also follows the scheme of model (i). In such a case. however. the total amount of incorporated In and the segregation coefficient would be highly correlated parameters and could not be separated.

If model (i) describes a segregated In-distribution. then how can the XSW results for the low- $T_{y}$ MOVPE samples be explained? It is possible that at the lower temperatures the InAs grows in a 3-dimensional mode, and forms islands with greater than 1 ML height. even prior to GaAs overgrowth. The direct observation of such islands would be difficult since the island heights would still be quite small. but could perhaps be achieved using atomic-resolution scanning probe microscopy.

The XSW and PL results for the I ML MOVPE samples would also be consistent with the incorporation of more than $\mathrm{ML}$ of InAs at the lower growth temperatures. and an In.As incorporation rate which decreases with increasing $T_{g}$. In homoepitaxy. Watkins et al. [138] observed the opposite behaviour. i.e. an increasing In.As growth rate with increasing $T_{g}$. For the SQW growth sequences the heteroepitaxial strain likely affects the In incorporation, so the homoepitaxy results do not preclude the possibility of excess In in the MOVPE samples. It is not possible to determine the In incorporation with sufficient precision to address this issue. The next section describes the results of X-Ray absorption measurements performed at SSRL.

\subsection{XAFS Study}

This section will begin by describing the reflectivity scans used for sample alignment. The experimental XAFS data will then be presented. followed by a discussion of the data analysis. 


\subsubsection{Reflectivity Measurements}

The sample crystals were aligned in the GIXAS apparatus as discussed in Section 6.T. Reflectivity ( $\left.R=I_{1} / I_{0}\right)$ measurements were made as a function of glancing angle. $\theta$, while simultaneously measuring the In $K_{u}$-fluorescence with the 13-element detector. A representative dataset obtained at $200 \mathrm{eV}$ above the In K-edge $\left(E_{0}=27940 \mathrm{eV}\right)$ is shown in Figure 7.28 . The background In-fluorescence was subtracted from the experimental curve using a second curve obtained below the In Kiedge.

The figure also shows the results of simulations for the reflectivity and the clectric field intensity in the In layer based on the method of Sections 4.3.1 and 4.3.2. The simulations used the GaAs cap thickness measured by HRXRD (92 A) for this sample. The theoretical curves were given a geometrical correction to account for the variation in the sample cross section illuminated by the bearn. Figure 6.12 shows that for $\sin \theta<h / L$ the reflectivity is slightly reduced because the beam height. $h$. is larger than the effective sample height, although the entire sample surface is illuminated by the beam. For $\sin \theta>h / L$ the reflectivity is unaffected by geometric effects. However. the beam can not illuminate the whole sample surface, and so the In fluorescence is slightly reduced. The simulated curves in Figure 7.28 also include a Ciaussian correlation function ( $\mathrm{FWHM}=19 \mathrm{mdeg}$ ) to account for the beam divergence.

Following sample alignment and reflectivity measurements, the samples were positioned at angles greater than the critical angle for the polarization-dependent X.AFS measurements. For the measurements presented here, incidence angles near $\theta=0.2^{\circ}$ were used. XAFS measurements were made by varying the photon energy according to Table 6.4 and recording the incident and reflected intensities, as well as the In $K_{\mathrm{u}}$-fluorescence.

\subsubsection{XAFS Analysis: Preliminaries}

The processing of raw XAFS data involves deglitching to remove Bragg peaks. and the summation of all scan data in a given series. This was accomplished using APL (A Programming Language) routines developed by Andrew Seary. The EXAFS, I, was then separated from the data using the AUTOBK program [139]. This involved 


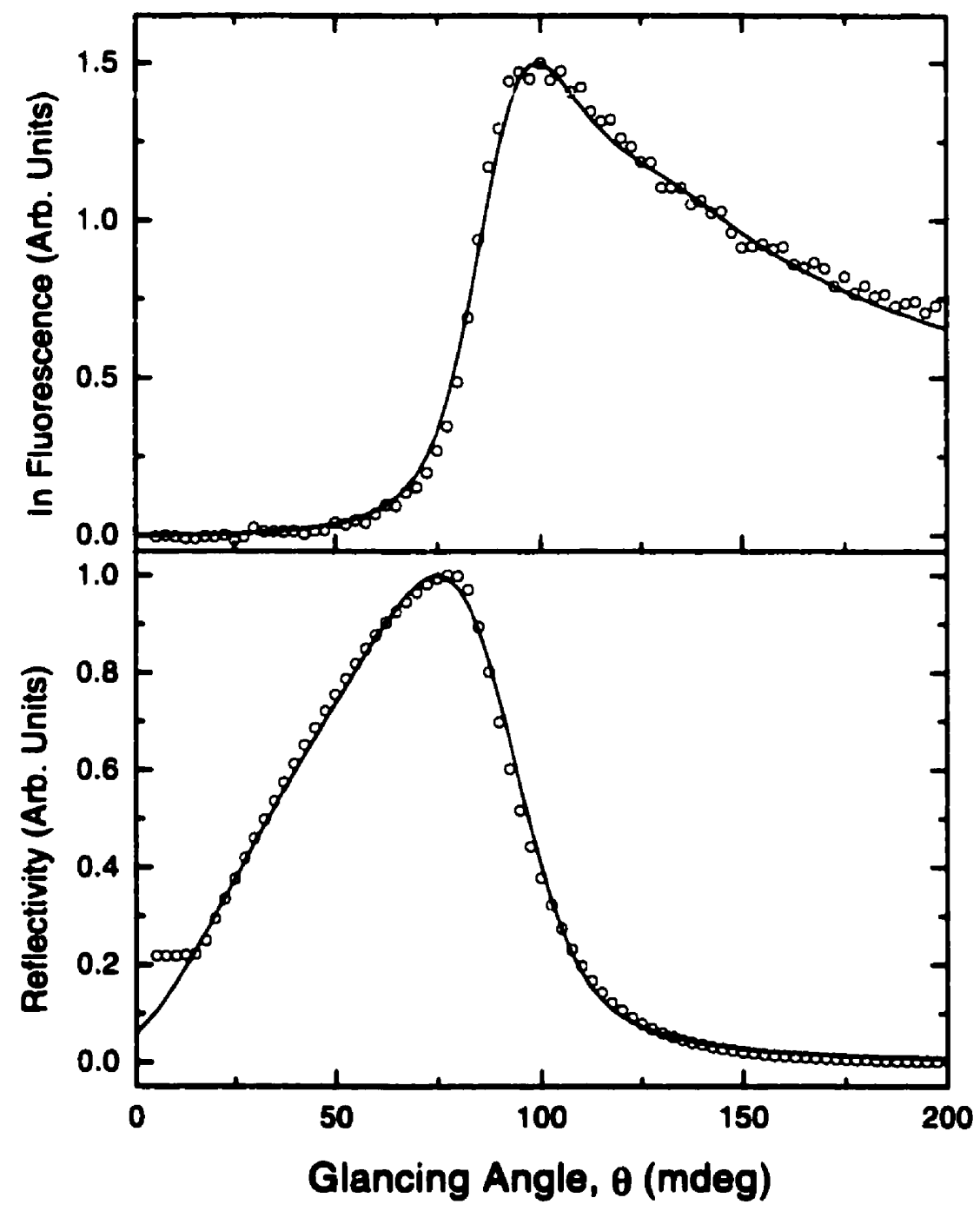

Figure 7.28: (a) Experimental reflectivity (circles) for the $1 \mathrm{ML}$ InAs SQW grown at $500{ }^{\circ} \mathrm{C}$ measured at $28140 \mathrm{eV}$ in the $E_{\|}$-polarization. (b) The In $\mathrm{K}_{\alpha}$-fluorescence yield (circles) measured for the same sample. Each curve also shows the results of simulations as described in the text. 
pre-edge background removal using a linear fit: normalization to the edge jump to put the data on a per-atom basis: and postedge background removal. In the last step a smoothly-varying background function which approximates the absorption from an isolated absorbing atom. $\mu_{0}(E)$, was subtracted from $\mu(E)$ to give $\backslash(E)$ (see Equation 4.4).

AUTOBK uses a fourth-order polynomial spline with knots equally spaced in $k$ space to minimize the low- $R$ background in the Fourier transform (FT) of $k \backslash(k)$. A good background subtraction will give a FT containing almost no low- $R$ components aside from leakage from the first shell. For the In K-edge XAFS. the AUTOBK program was used to minimize the background below the first shell automatically by comparing the data with a reference $(\mathrm{FEFF} 6 \mathrm{~b})$ spectrum. ACTOBK subtracts a trial spline function from the data and then transforms the data into $R$-space. The spline is then allowed to vary iteratively until the low- $R$ region is optimized in comparison with the reference. In this background subtraction ACTOBK was allowed to adjust the edge energy. $E_{0}$. to reduce the low- $R$ contributions. rather than holding $E_{0}$ fixed at the inflection point (the point on the edge where $d^{2} \mu(E) / d E^{2}=0$ ). This approach will be discussed in Section 7.7.4.

Figure 7.29 shows the In K-edge XAFS from an InAs reference powder. These transmission data were obtained at beamline $\mathrm{X} 23 \mathrm{~A} 2$ using $\mathrm{Si}(311) \mathrm{MC}$ crystals. Since the structure of the reference is well known, it provides a useful test of the analysis procedure. Superimposed on the data is the ACTOBK spline background. For the large $k$-space range of these data the spline contained $1 i$ knots. For the InAs SQW samples 1.5 knots were used. The dataset shown in Figure 7.29 has been adjusted by removal of the pre-edge line and normalized by the edge jump. Following this procedure the detailed data analysis may proceed.

\subsubsection{EXAFS Analysis: Curve Fitting}

As discussed in Chapter 4, experimental EXAFS $\chi(k)$ data may be analyzed using either a reference spectrum or a theoretical model. The InAs SQW samples were 


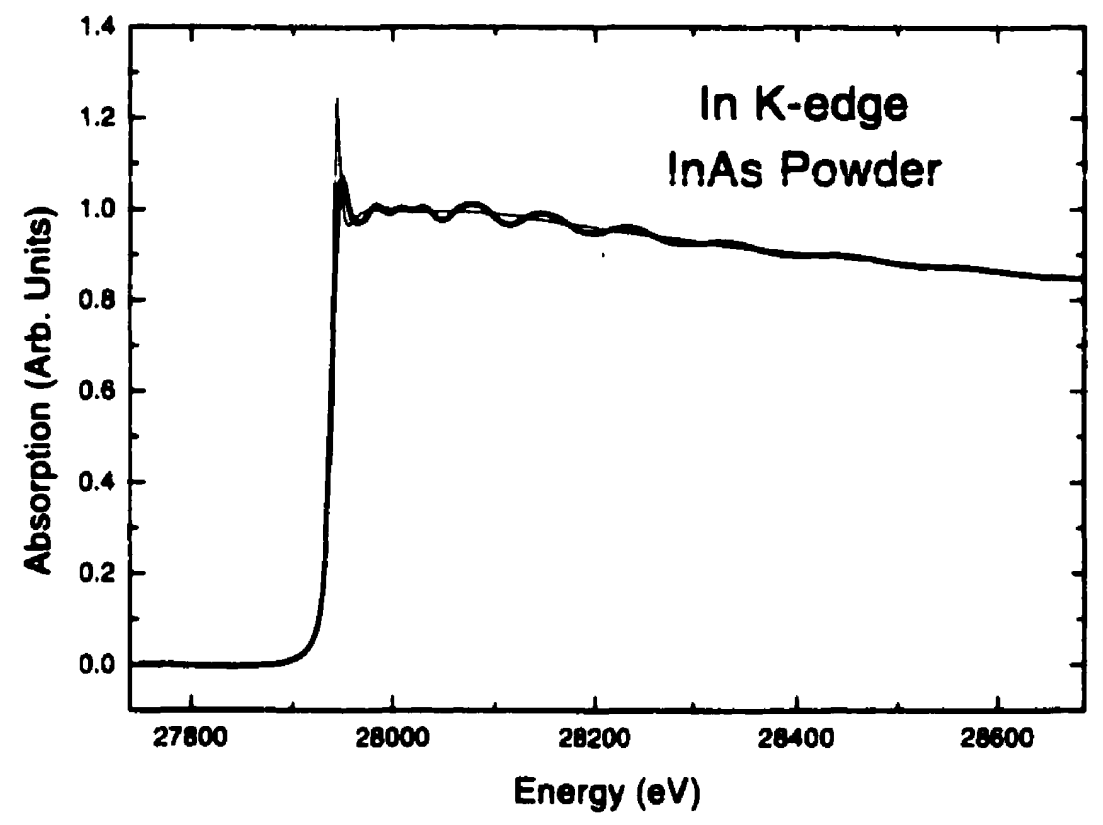

Figure 7.29: In li-edge XAFS from an InAs powder sample obtained in transmission at beamline $\times 23 \mathrm{~A} 2$ at the NSLS (heavy line) and the AUTOBK background (light line). The pre-edge linear background was removed from both curves and the curves were normalized by the edge jump.

analyzed using both methods for comparison. For the InAs reference powder the InAs bond lengths were fitted using a FEFF6 $\mathrm{b}$ model to evaluate the precision of the fits obtained with this method.

The FEFF model was produced using the InAs lattice constant. $a_{I n A s}=6.0584 \mathrm{~A}$. and the zincblende structure. In the model an In atom at the center of the crystal was defined as the absorber and the EXAFS was calculated. The first shell contains four As atoms located a distance of $2.623 \AA$ from the absorbing atom. FEFF6 $b$ uses a curved-wave model to calculate the phase shifts, effective scattering amplitudes, and single and multiple scattering EXAFS and XANES spectra [44].

In this thesis all EXAFS curve-fitting was done in $k$-space, although fitting in 
$R$-space is also possible. After background removal the data were transformed into $R$ space using a $10 \%$ Gaussian window function. The FT magnitude for the experimental InAs reference data appearing in Figure 7.30 , shows that the EXAFS consists mainly of an In-As first shell peak. Second and higher shells are smaller by more than an order of magnitude. This suggests that the determination of bond-lengths and coordination numbers beyond the first shell is essentially impossible for such samples. Given that the data shown in Figure 7.30 were obtained for a concentrated powder specimen the second shell analysis for dilute samples (e.g. InAs MLs in Ga.As) is even more impractical.

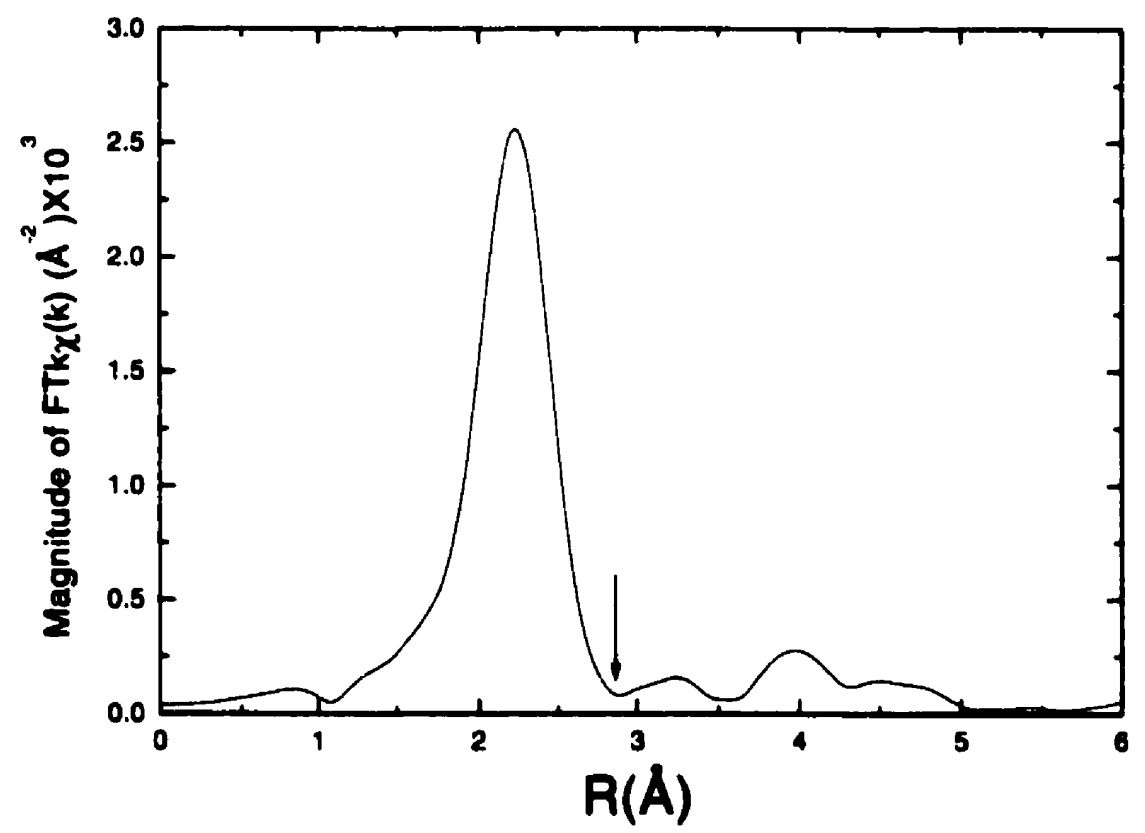

Figure 7.30 : Fourier transform of the $k$-weighted In K-edge EXAFS from the InAs powder sample. A $10 \%$ Gaussian window was used in the transform over the range $k=0.52 \rightarrow 16.15 \AA^{-1}$. The arrow indicates the maximum $R$-value used in the Fourierfiltering.

The next analysis step involved Fourier-filtering the data by selecting a range 
in $R$-space to include only the first shell. In Figure $i .30$ the data from $0.03 \AA$ to $2.86 \AA$ were included. This limited range of data was then back-transformed into $k$-space where the curve-fitting determines the first shell structure.

From Chapter 4, the EXAFS, $\backslash(k)$, for $\mathrm{K}$-edge absorption is expressed as

$$
l(k)=\sum_{i} B_{i}(k) \sin \left[2 k R_{1}+2 \delta(k)+\phi_{i}(k)\right] .
$$

where the sum is taken over all atoms, $i$, located at distances $R_{i}$ from the absorbing atom. and the phase shifts. $\delta(k)$ and $\phi_{i}(k)$. arise from the interaction between the absorber and the backscatterer, respectively. The amplitude function, $B_{i}\left(k_{i}\right)$. was defined as

$$
B_{i}(k)=\frac{N_{i}^{*}}{k R_{i}^{2}} S_{0}^{2} F_{i}(k) e^{-2 R_{i} / N_{1}(k)} \epsilon^{-2 k^{2} \sigma_{i}^{2}} .
$$

where

$$
N_{i}^{*}=3 N_{i} \cos ^{2}\left(\theta_{i}\right)
$$

Here $N_{i}$ is the number of atoms of type $i$ located at an average distance $R_{i}$ from the absorber and the other quantities were defined in (hapter 4.

Figure 7.31a) shows the Fourier-filtered first shell (FFFS) superimposed on the raw EXAFS data. The observation that the data consist mainly of first-shell EXAFS is clearly confirmed. Figure $7.31 \mathrm{~b}$ ) shows the FFFS data, as well as the fit using the FEFF model. The data were least-squares-fitted using the theoretical amplitude. $B_{i}(k)$, and phase. $\left(2 \delta(k)+\phi_{i}(k)\right)$ for the In-absorber and As-first-shell backscatterer. In the fits the variable parameters were $S_{0}^{2}, R, \sigma^{2}$ and $\lrcorner E_{0}$, which allows for an offset between the theoretical and experimental values of $E_{0}$, obtained from AUTOBK. The fits were carried out using only the data for $k>4 A^{-1}$ to minimize the effect of the energy shift on the In-As bond length, which is most sensitive to such a discrepancy. The fitting was done using the WINXAS software package. ${ }^{1}$

The results of the fitting are: $S_{0}^{2}=1.03 \pm 0.07$ and $\sigma^{2}=0.004 i \pm 0.005$, while the In-As bond length is $R=2.625 \pm 0.004 \AA$. The error bars indicate the values which double the squared residuals in each parameter when the other values were allowed to float. Figure 7.31 clearly shows the agreement between the data and the FEFF

\footnotetext{
'WINXAS 7.0 (C)Thorsten Ressler.
} 


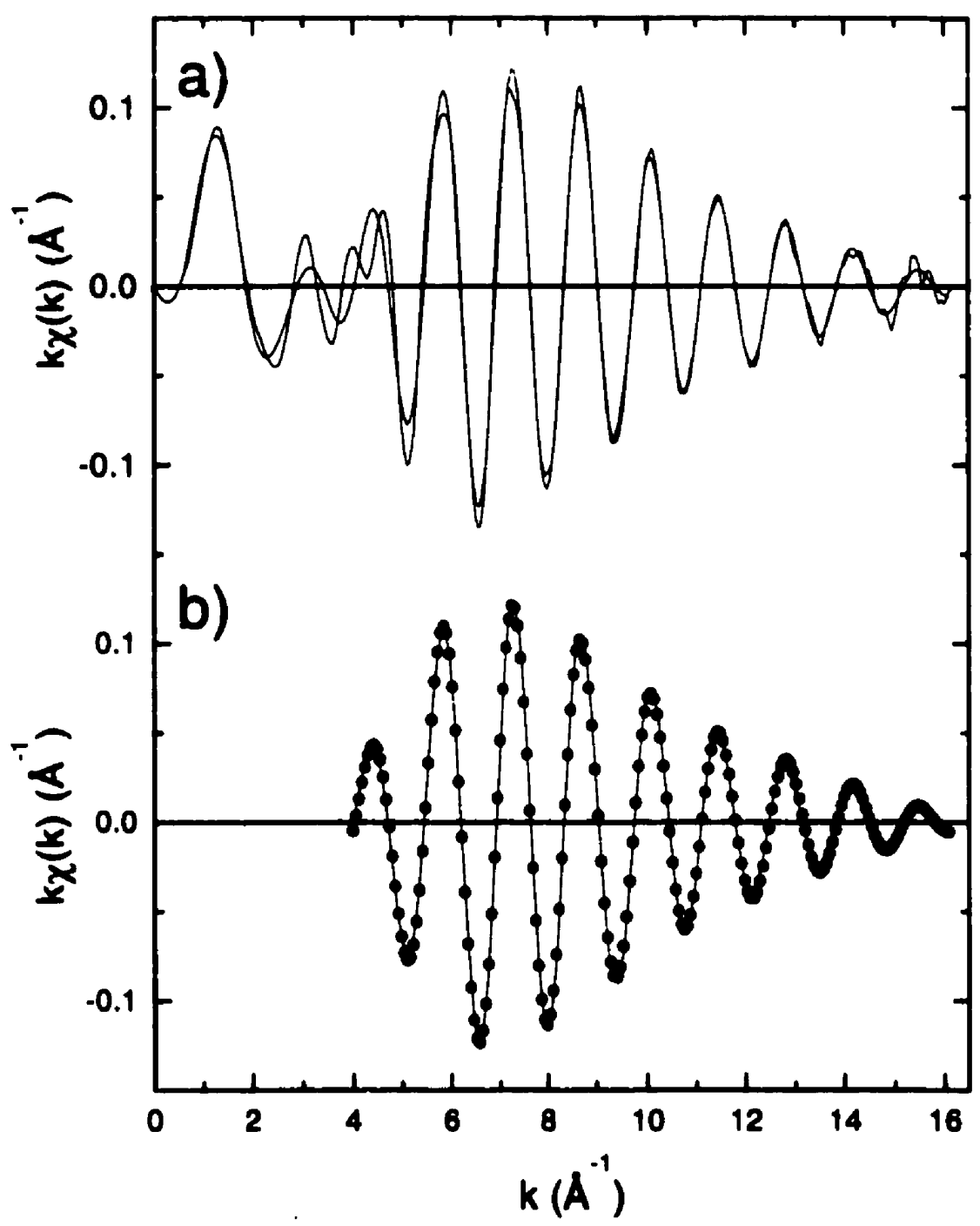

Figure 7.31 : a) Raw EXAFS data from the InAs powder specimen and the Fourierfiltered first-shell contribution, due to In-As bonds. b) Fourier-filtered first-shell data (solid circles) and the fit to the data obtained with the FEFF predictions. 
model. In fact, the agreement between the fitting result and the known In-As bond length (2.623 $\AA$ ) is outstanding. It must be noted that results of this accuracy were not obtained if the data were fitted to lower $k$-values.

Given this basis in EXAFS analysis we now consider the results for single ML samples, obtained in the glancing-incidence geometry.

\subsubsection{EXAFS Analysis: SQW Samples}

Figure 7.32 shows XANES spectra for the ML samples in the parallel and perpendicular polarizations, as well for the InAs powder. The XANES is related to the electronic structure and bonding geometry of the absorbing atom, so it is reassuring to see the agreement between the data for different samples. and between the two polarizations.

The background subtraction for the 1 ML samples again used AITOBK, with a reference $\backslash(k)$ obtained from a FEFF model for a single InAs layer in CiaAs. The data were then Fourier-filtered to fit the first shell data. The magnitudes of the Fourier-transformed data are shown in Figure 7.33 .

In the parallel geometry, the FTs are qualitatively similar between the samples grown at 500 and $600{ }^{\circ} \mathrm{C}$, consisting primarily of a large first shell peak centred near $2.1 \mathrm{~A}$. The peak position is shifted downwards from the actual bond length because of the EXAFS phase shift (Equation 7.18 ). Small differences exist between the higherorder peak positions in the two samples. The first shell peaks in the $E_{\perp}$ geometry line up very well with one another. The $500^{\circ} \mathrm{C}$ sample is different from the other two in its second shell, however, and seems to exhibit a splitting. It is not clear whether this is a real effect or statistical artifact. The low- $R$ region exhibits a spurious feature related to the background. The nodes above and below the second shell line up very well in the three samples. In the InAs/GaAs SQW FEFF model, the central In absorbing atom is surrounded by an arrangement of backscatterers as listed in Tables $\mathbf{7 . 6}$ and $\mathbf{7 . \pi}$. The model used the MET to evaluate the In-As planar distance and the atomic locations were calculated from the geometry of the zincblende lattice.

Tables 7.6 and 7.7 follow the notation of Zabinsky et al. [44] to enumerate the 


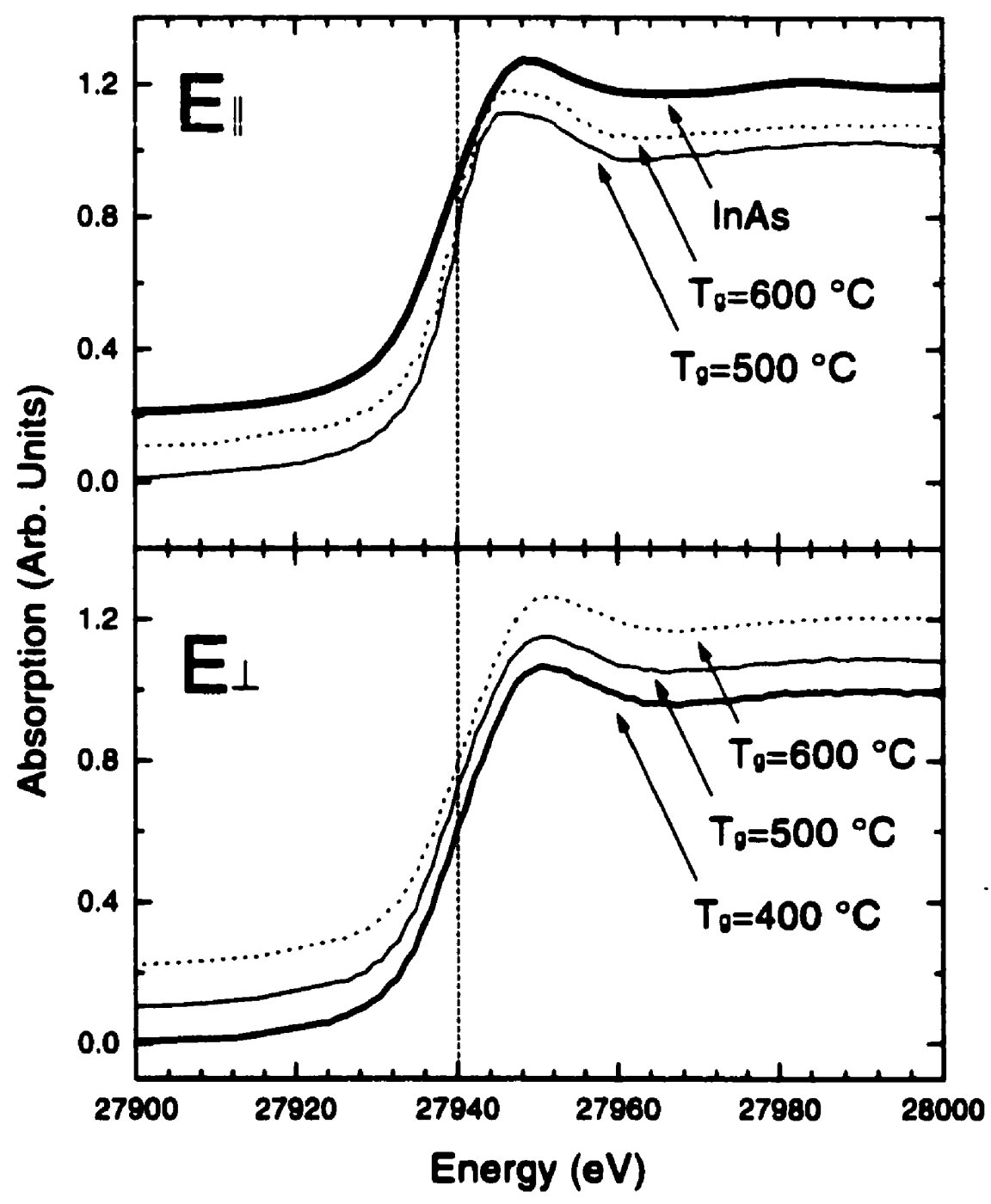

Figure 7.32: In K-edge XANES from the 1 ML InAs films, obtained in both the parallel $\left(E_{\|}\right)$and perpendicular $\left(E_{\perp}\right)$ geometries and growth temperatures as indicated. The top spectrum was obtained from the InAs reference powder in transmission. The spectra have been normalized and offset for clarity. The dotted vertical line denotes the edge energy. 


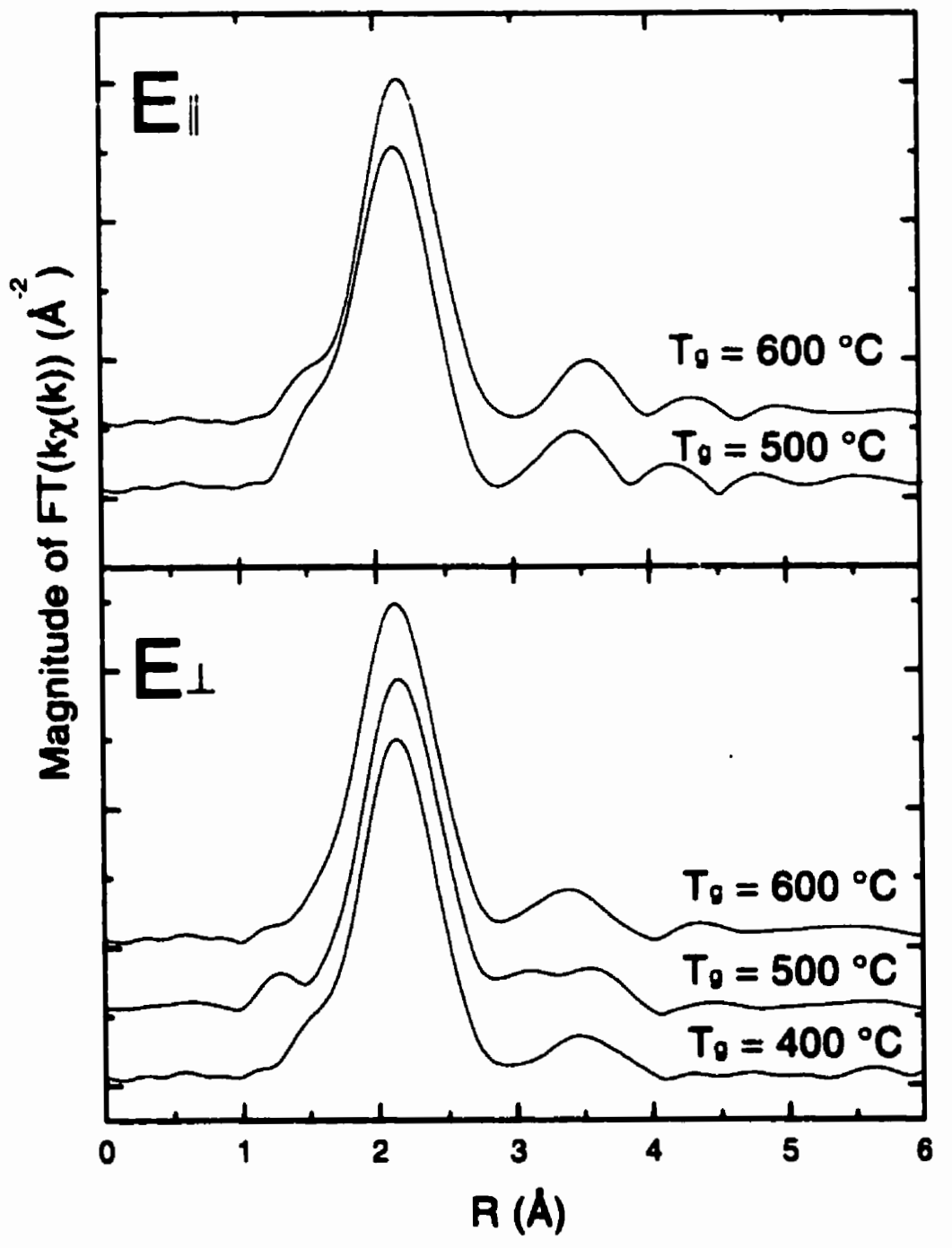

Figure 7.33: Fourier transform magnitudes for $k$-weighted In K-edge EXAFS in $E_{\|}$ (top curves) and $E_{\perp}$ polarizations (bottom curves) for $1 \mathrm{ML}$ samples and growth temperatures as indicated. A $10 \%$ Gaussian window was used in each transform over a range of about $k=0 . \bar{\tau} \rightarrow 12 \hat{A}^{-1}$. 


\begin{tabular}{|c|c|c|c|c|c|}
\hline Backscatterer & $\overline{\overline{R(A)}}$ & $\overline{\overline{C_{c w}}}$ & $\overline{\overline{N_{\Gamma}}}$ & $n$ & Type \\
\hline As & 2.576 & & 2 & 2 & SS, first shell \\
\hline As & 2.576 & 100.00 & 2 & 2 & SS, first shell \\
\hline$\overline{I n}$ & $3.99 \bar{i}$ & 61.13 & 2 & 2 & SS, second shell \\
\hline In & 3.997 & 0.69 & 2 & 2 & SS, second shell \\
\hline $\mathrm{Ga}$ & 4.150 & 35.16 & $\overline{8}$ & 2 & SS, third shell \\
\hline & 4.575 & & 2.2 .4 .4 & 3 & MS \\
\hline & 4.587 & & 8.8 & 3 & MS \\
\hline & 4.729 & & $\overline{8}$ & 3 & .MS \\
\hline As & 4.755 & 36.10 & 4 & 2 & SS. fourth shell \\
\hline$\overline{A s}$ & 4.755 & 8.91 & 4 & 2 & SS. fourth shell \\
\hline$\overline{A s}$ & 4.879 & 0.07 & 2 & 2 & SS. fifth shell \\
\hline As & 4.879 & 3.89 & 2 & 2 & SS, fifth shell \\
\hline
\end{tabular}

Table 7.6: The most important paths in the InAs SQW FEFF calculation for the parallel geometry. Here $R$ is the effective path length: $C_{c w}$ is the importance in percent of each path relative to the first SS path: the degeneracy. $\nu_{r}$. is the number of equivalent paths; $n$ indicates the number of legs for each path: and path types include "SS" single scattering and "MS" multiple scattering. Some of the MS contributions involve several different paths (with degeneracies shown) and these are not listed completely. The $C_{c w}$ contribution of the first pair of $A$ s atoms in the first shell is negligible, but FEFF erroneously assigns a value of 100.00 .

paths out to $5 \mathrm{~A}$. Note that the degeneracy of some of the paths is lifted when the $E_{\|}$orientation is used. The FEFF calculation includes the polarization by aligning the electric vector along the (110) or (001) crystal axes. for the $E_{\|}$and $E_{\perp}$ cases. respectively. In the experiment the $E_{\|}$-orientation was not exactly along the (110)axis because the sample was rotated slightly to manipulate the unwanted Bragg peaks. A simple geometric calculation verifies that the rotation does not affect the results. and that only two of the first shell As atoms contribute to the EXAFS. Multiple scattering refers to any path more complicated than a simple absorber-backscatterer interaction. In the following the discussion will be limited to the first shell, containing In-As bonds.

Figures 7.34 and 7.35 show the raw EXAFS data and the results of fitting the FFFS using the FEFF phases and amplitudes. The fits were carried out with the variable parameters $S_{0}^{2}, R, \sigma^{2}$ and $\Delta E_{0}$. Results of the fitting for the $1 \mathrm{ML}$ samples 


\begin{tabular}{|c|c|c|c|c|c|}
\hline \hline Backscatterer & $R(A)$ & $C_{c w}$ & $N_{\Gamma}$ & $n$ & Type \\
\hline As & 2.576 & 100.00 & 4 & 2 & SS, first shell \\
\hline In & 3.997 & 1.05 & 4 & 2 & SS, second shell \\
\hline $\mathrm{Ga}$ & 4.150 & 62.85 & 8 & 2 & SS, third shell \\
\hline & 4.575 & & 4.8 & 3 & MS \\
\hline & 4.587 & 13.48 & 16 & 3 & MS \\
\hline & 4.729 & 5.85 & 8 & 3 & MS \\
\hline As & 4.755 & 8.93 & 8 & 2 & SS, fourth shell \\
\hline As & 4.879 & 29.88 & 4 & 2 & SS. fifth shell \\
\hline \hline
\end{tabular}

Table $7 . i$ : The most important paths in the InAs SQW FEFF calculation for the perpendicular geometry. Here $R$ is the effective path length: $C_{c u}$ is the importance in percent of each path relative to the first SS path; the degeneracy. $\nu_{\Gamma}$. is the number of equivalent paths; $n$ indicates the number of legs for each path; and path types include SS and MS. The first MS contribution involves two different paths (with degeneracies shown).

are tabulated in Table 7.8 . In all cases the experimental In-As bond lengths agree with the expected value $(R=2.576 \mathrm{~A}$ ) within the uncertainties in the fitting. Similar (In $\mathrm{L}_{3}$-edge) results were obtained by Woicik et al. [2]. although the $k$-space range was limited to $4 \rightarrow \pi .2 \mathrm{~A}^{-1}$ by the intervening $L_{2}$-edge. The $K$-edge measurements presented here have the advantage of an extended $k$-space range which gives higher precision.

It is well known that $\Delta E_{0}$ and $R$ can be highly correlated parameters [140]. In the background subtraction, AUTOBK was allowed to adjust $E_{0}$ to minimize the low- $R$

\begin{tabular}{|c|c|c|c|c|c|c|c|}
\hline \hline Mode & Sample & $S_{0}^{2}$ & $R(\AA)$ & $\sigma^{2}\left(\AA^{2}\right)$ & $N^{e}$ & $R^{e}(\AA)$ & $N^{e} / N^{t h}$ \\
\hline $\mathrm{E}_{\|}$ & $500^{\circ} \mathrm{C}$ & $1.32 \pm 0.16$ & $2.571 \pm 0.007$ & $0.0050 \pm 0.0008$ & $4.6 \pm 0.4$ & $2.568 \pm 0.006$ & $1.3 \pm 0.1$ \\
\hline & $600^{\circ} \mathrm{C}$ & $1.01 \pm 0.06$ & $2.575 \pm 0.004$ & $0.0028 \pm 0.0004$ & $4.7 \pm 0.6$ & $2.573 \pm 0.008$ & $1.3 \pm 0.2$ \\
\hline $\mathrm{E}_{\perp}$ & $400^{\circ} \mathrm{C}$ & $0.85 \pm 0.08$ & $2.574 \pm 0.006$ & $0.0035 \pm 0.0006$ & $4.6 \pm 0.5$ & $2.571 \pm 0.007$ & $0.97 \pm 0.10$ \\
\hline & $500^{\circ} \mathrm{C}$ & $0.98 \pm 0.13$ & $2.585 \pm 0.008$ & $0.0050 \pm 0.0010$ & $4.4 \pm 0.6$ & $2.581 \pm 0.008$ & $0.92 \pm 0.12$ \\
\hline & $600{ }^{\circ} \mathrm{C}$ & $0.83 \pm 0.09$ & $2.571 \pm 0.006$ & $0.0032 \pm 0.0007$ & $4.7 \pm 0.6$ & $2.567 \pm 0.009$ & $0.99 \pm 0.13$ \\
\hline \hline
\end{tabular}

Table 7.8: Fit parameters obtained from the experimental In-As first shell EXAFS. The data were fitted using both FEFF and the empirical InAs standard as described in the text. The error bars indicate the values which double the squared residuals in each parameter when the other values were allowed to float. 
background of the fluorescence EXAFS with respect to the FEFF standard. This led to a choice of $E_{0}$ slightly different than the inflection point (up to a difference of nearly $8 \mathrm{eV}$ in the SQW samples). This occurs, in part, because of the inaccuracy of FEFF in estimating the correct position of $k=0$. In the EXAFS curve-fitting. the variable parameter $\Delta E_{0}$ compensates for this discrepancy. To investigate the effect of this energy shift on the bond-lengths the analysis procedure was repeated holding $E_{0}$ fixed at the inflection point in the ACTOBK background removal. When the resulting SQW EXAFS datasets were then fitted using the FEFF models, it was found that the $\Delta E_{0}$ shifts were reasonably similar for all samples (between 1.8 and $4.5 \mathrm{el}$ ) and the best-fit In-As bond lengths were within the error bars quoted in Table 7.8 .

Likewise. the data were fitted in a comparative manner by using an "internal reference". "sing the best-fit $\Delta E_{0}$ for one of the samples, the datasets for the remaining samples were curve-fitted with this fixed $\lrcorner E_{0}$. This procedure was repeated several times, by rotating through in the selection of the internal reference. Again the bond lengths agreed with the results shown in Table 7.8 within the quoted error bars. These alternative approaches helped confirm the validity of the analysis approach leading to the results in Table 7.8 .

In addition to the FEFF fits, the present data were fitted using the InAs reference $\backslash(k)$ as an empirical phase, $\phi(k)$, and amplitude. $|f(k)|$. standard.

$$
\gamma(k)=\frac{N^{e}}{k\left(R^{e}\right)^{2}}|f(k)| \sin \left[2 k R^{e}+\phi(k)\right] .
$$

In this case the variable parameters in the fits were the coordination number. $V^{e}$. the In-As bond length, $R^{e}$, and an offset $\Delta E_{0}$. Note that in this case $N^{e}$ contains contributions from the coordination number. as well as the from the EXAFS DebyeWaller factor, $e^{-2 k^{2} \sigma^{2}}$. The results are given in Table 7.8 from which it is seen that the bond lengths obtained using the empirical method agree very well with those obtained using the theoretical calculation. There does not appear to be any connection between the In-As bond lengths and the growth temperature.

The parameter $N^{\text {th }}$ in Table 7.8 refers to the theoretical number of nearestneighbours based on the geometry $\left(N^{t h}=N_{i}^{*}\right)$. According to Equation 7.20 this 
is calculated from the angle, $\theta$, between the incident electric vector and the direction of the bond. Using the MET to predict the In-As plane spacing. the geometry gives $N^{\text {th }}=3.61$ in the parallel geometry and $N^{t h}=4.78$ in the perpendicular geometry. These values take into account the 2 and 4 atoms which contribute in the $E_{\|}$and $E_{\perp}$-orientations. respectively.

Although the bond length fitting results agree quite well with the expected values. the effective coordination numbers show larger discrepancies. It is generally more difficult to obtain precise $N$-values because they depend on the EXAFS amplitude and so normalization errors directly affect the results. Also, there is an outstanding question of the transferability of the amplitude and phase clata obtained in transmission. when one attempts to use this to model glancing-incidence data. For the $E_{\|}$-orientation the fitting results are larger than expected. and for the $E_{\perp}$-orientation the results are slightly lower than expected.

In the FEFF fitting the parameters $S_{0}^{2}$ and $\exp \left(-2 k^{2} \sigma^{2}\right)$ can also be correlated [140]. Each of these parameters affects the EXAFS amplitude making it slightly difficult to separate the two contributions. although the exponential term does have a $k$ dependence. These parameters may be studied in more detail using temperaturedependent measurements, for example.

If each InAs SQW sample contained In atoms arranged in a single layer. then the XSW results (Section 7.6.4) might be interpreted in terms of reduced bond length compression at lower values of $T_{g}$. Assuming coherency with the GaAs substrate. the experimental values of $P^{H}$ would then correspond to $\mathrm{In}-\mathrm{As}$ bond lengths of $R=2.612 \AA\left(400{ }^{\circ} \mathrm{C}\right) . R=2.594 \AA\left(500^{\circ} \mathrm{C}\right)$ and $R=2.576 \AA\left(550,600{ }^{\circ} \mathrm{C}\right)$. Clearly the EXAFS bond lengths do not support this single-layer interpretation for the lower values of $T_{g}$.

The next section presents the PL data which are used to evaluate the optical properties of the SQW samples and for comparison with the structural (XSW) studies. 


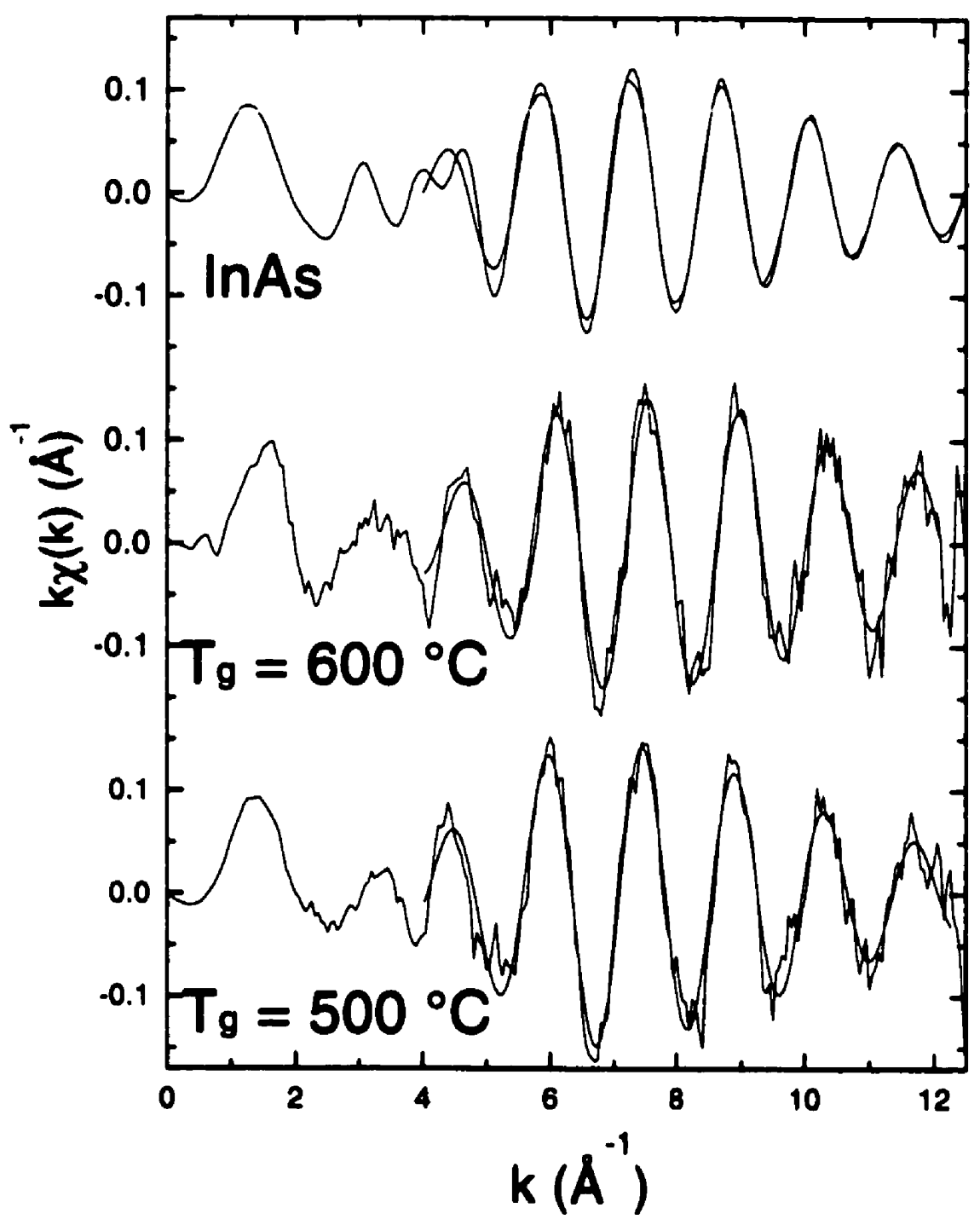

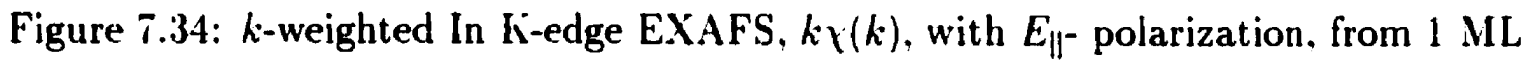
InAs films and growth temperatures as indicated. The top spectrum was obtained using an In.As reference powder in transmission. Superimposed on the data are the fits to the Fourier filtered first-shell contributions, which correspond to the In-As near neighbour bond lengths. 


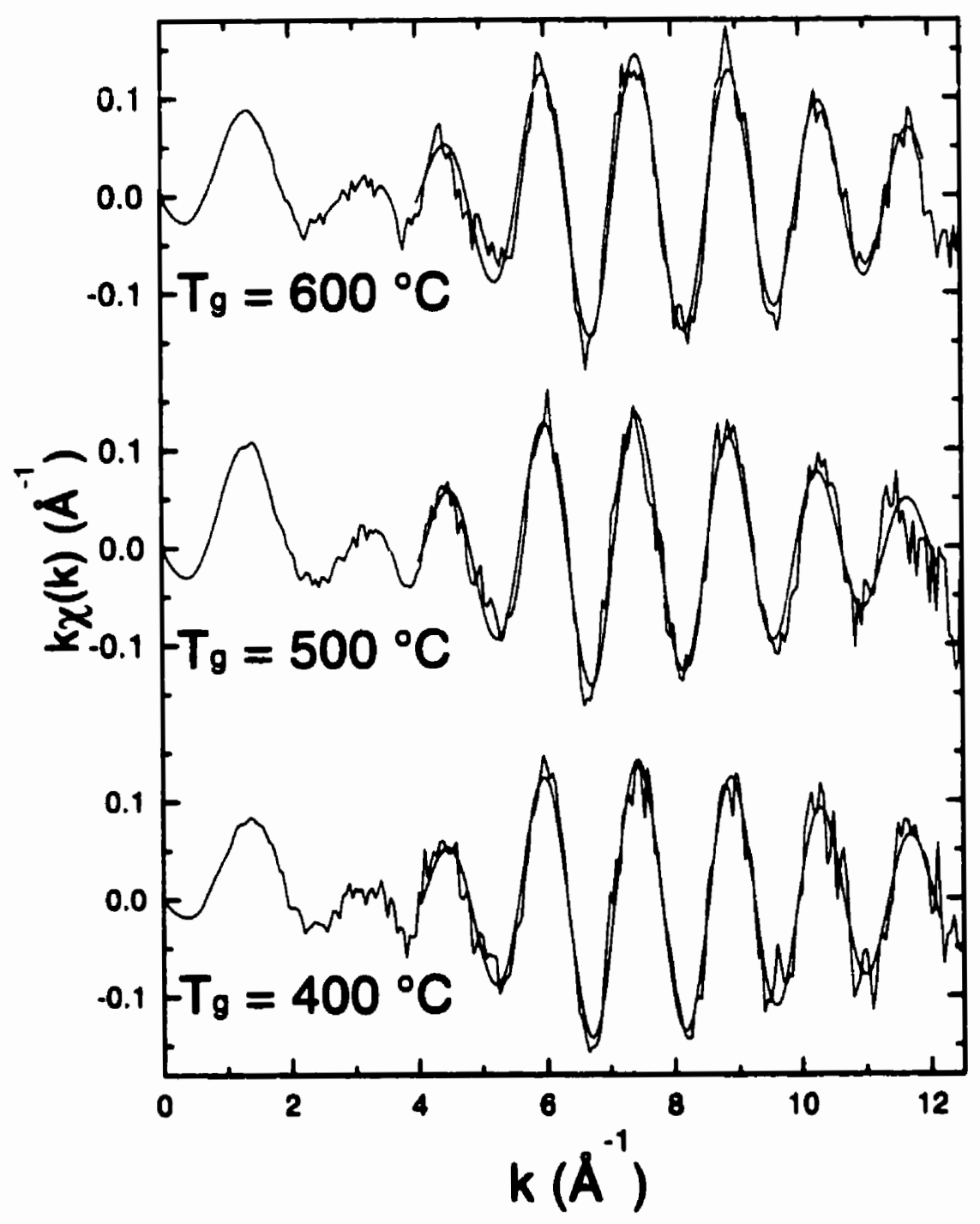

Figure $7.35: k$-weighted In K-edge EXAFS, $k \gamma(k)$, with $E_{\perp}$-polarization, from $1 \mathrm{ML}$ InAs films and growth temperatures as indicated. Superimposed on the data are the fits to the Fourier filtered first-shell contributions, which correspond to the In-As near neighbour bond lengths. 


\subsection{PL Results}

The PL spectra for the 1 ML samples are shown in Figure 7.36 . For the sample grown at $400^{\circ} \mathrm{C}$ the spectrum shows very weak In-excitonic luminescence. The feature labeled 'DAP' represents donor-acceptor pair $(1.490 \mathrm{eV})$ and free-to-bound $(1.193 \mathrm{eV})$ transitions at carbon impuritics, resulting from incomplete TBAs decomposition which leads to carbon incorporation in the crystal. For the remaining samples, the PL emission energies increase with growth temperature. while the linewidths generally decrease. An exception is the sample with $T_{y}=5.30^{\circ} \mathrm{C}$. exhibiting particularly sharp luminescence.

For the 1/2 ML samples (Figure 7.37 ). $400^{\circ} \mathrm{C}$ is again seen to be unsuitable for good optical material, despite the more promising $\mathrm{XSW}$ results. The spectrum is dominated by the carbon impurity features. The brightest 1/2 MIL In-luminescence is observed for the sample with $T_{g}=600^{\circ} \mathrm{C}$.

Figure $i .38$ shows a comparison between the structural and optical properties. The data suggest a correspondence between the decreasing $P^{H}$ and the increasing PL peak energies as a function of growth temperature in the $1 \mathrm{ML}$ samples. As well, the PL linewidths decrease dramatically with increasing $T_{g}$. This is mirrored somewhat by the increasing coherent fraction. These characteristics may be attributed to a trend towards more perfect. $\delta$-like InAs layers, being particularly evident in the $530-5550^{\circ} \mathrm{C}$ range.

Previous treatments [84] of the effect of In segregation on optical emission found that with decreasing $T_{g}$ the transitions are shifted towards lower energies and then saturate at minima which. the authors argued. correspond to minima of segregation. In that case, however, the transition energy vs. $T_{g}$ curves were concave up. From Figure 7.38 , our results do not reproduce these observations. Rather, the PL energies increase with increasing $T_{g}$. and may approach limiting behaviour at temperatures beyond those considered here. We point out that the curve is concave down in this case. Thus, these results do not necessarily indicate temperature-dependent segregation.

Previously in this work we showed that the large coherent positions observed by XSW in the low- $T_{g}$ samples were consistent with model (ii) which supposed In 


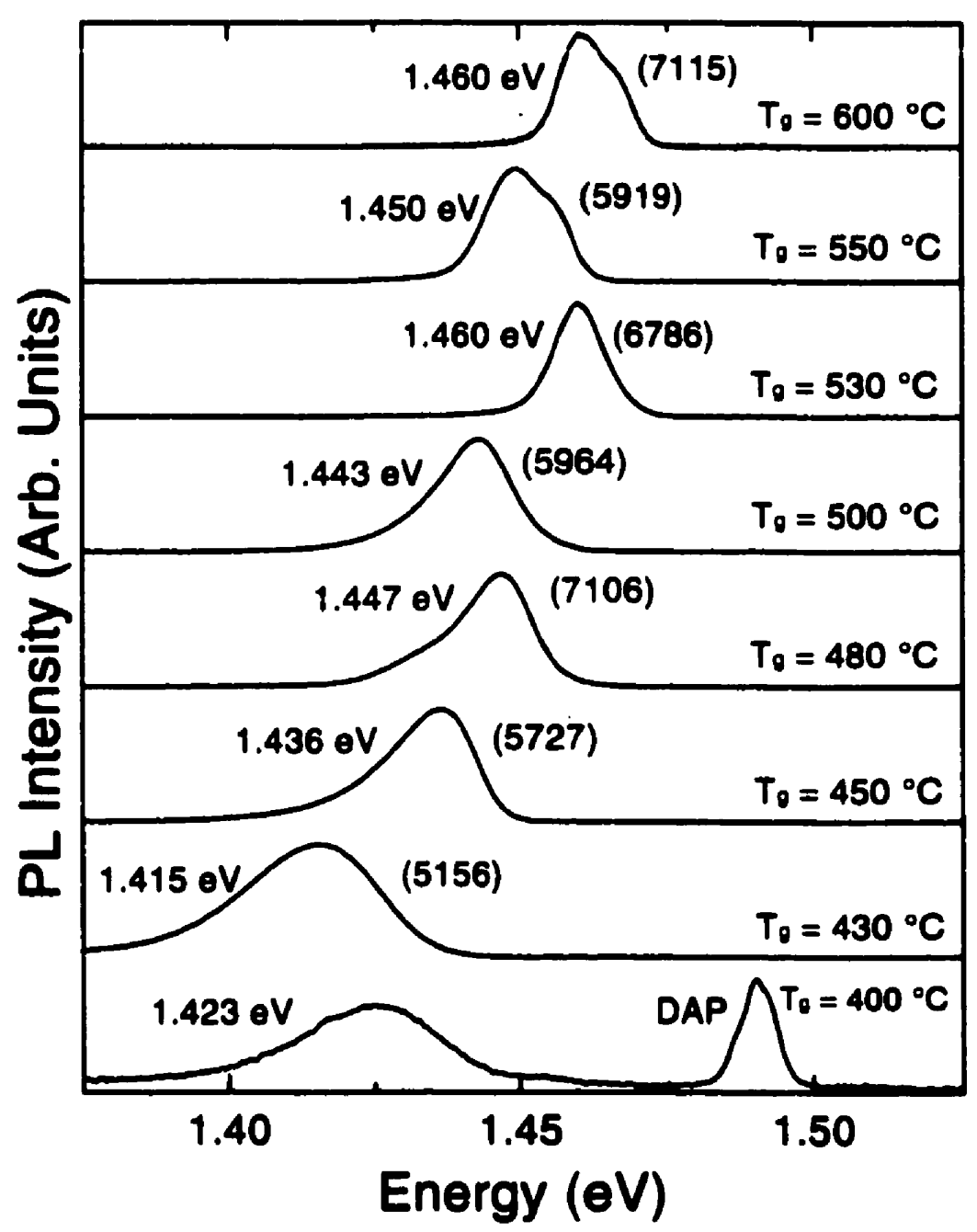

Figure 7.36: PL spectra for a series of $1 \mathrm{ML}$ InAs samples, with growth temperatures as indicated. The spectra were normalized to a peak height of unity and offset for clarity. The quantities in parentheses denote the absolute peak amplitudes prior to normalization. 


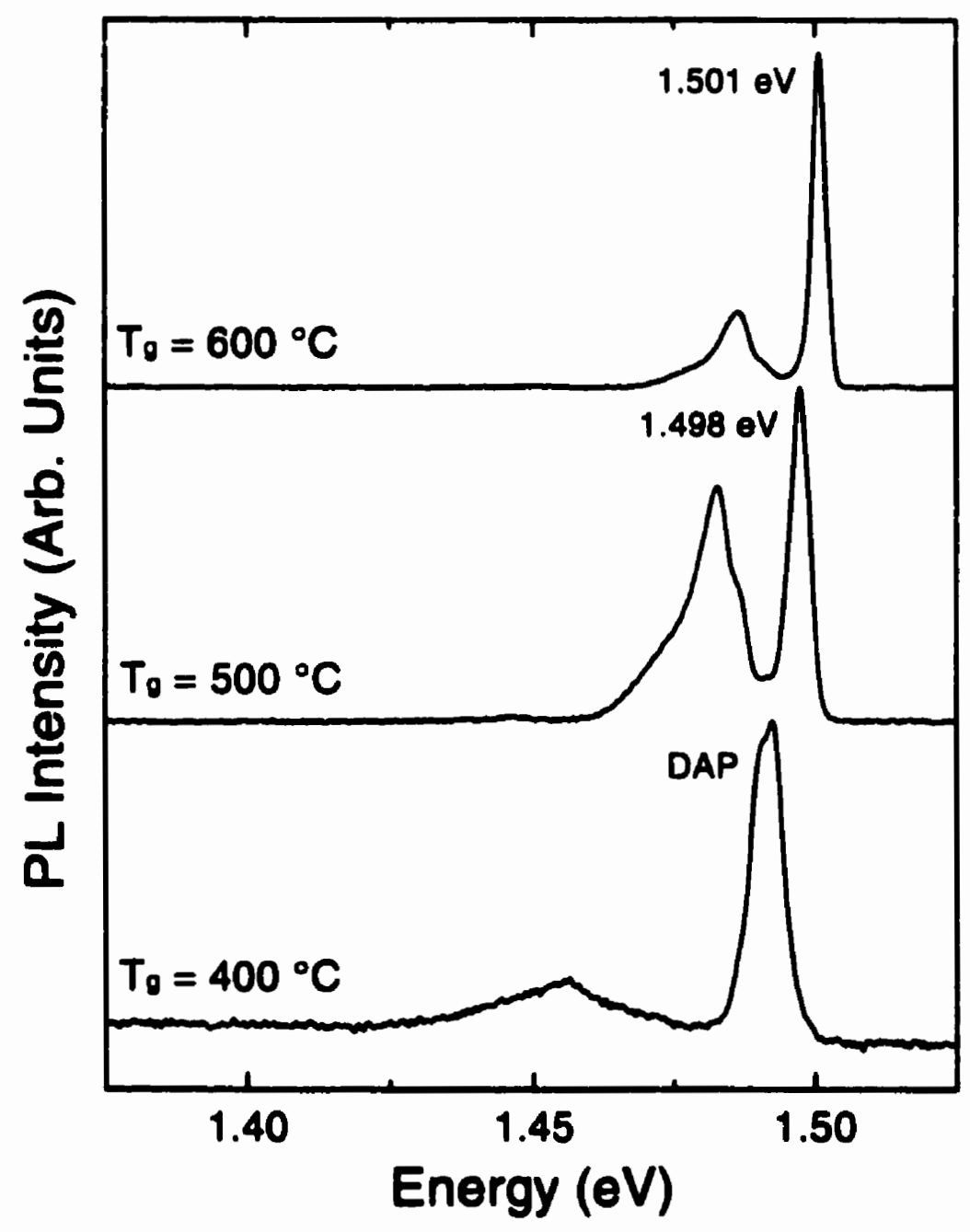

Figure 7.37: PL spectra for a series of 1/2 ML InAs samples, with growth temperatures as indicated. The spectra were normalized to a peak height of unity and offset for clarity. For the $500{ }^{\circ} \mathrm{C}$ and $600{ }^{\circ} \mathrm{C}$ samples the peak energies indicated refer to the more intense, higher energy peaks. 


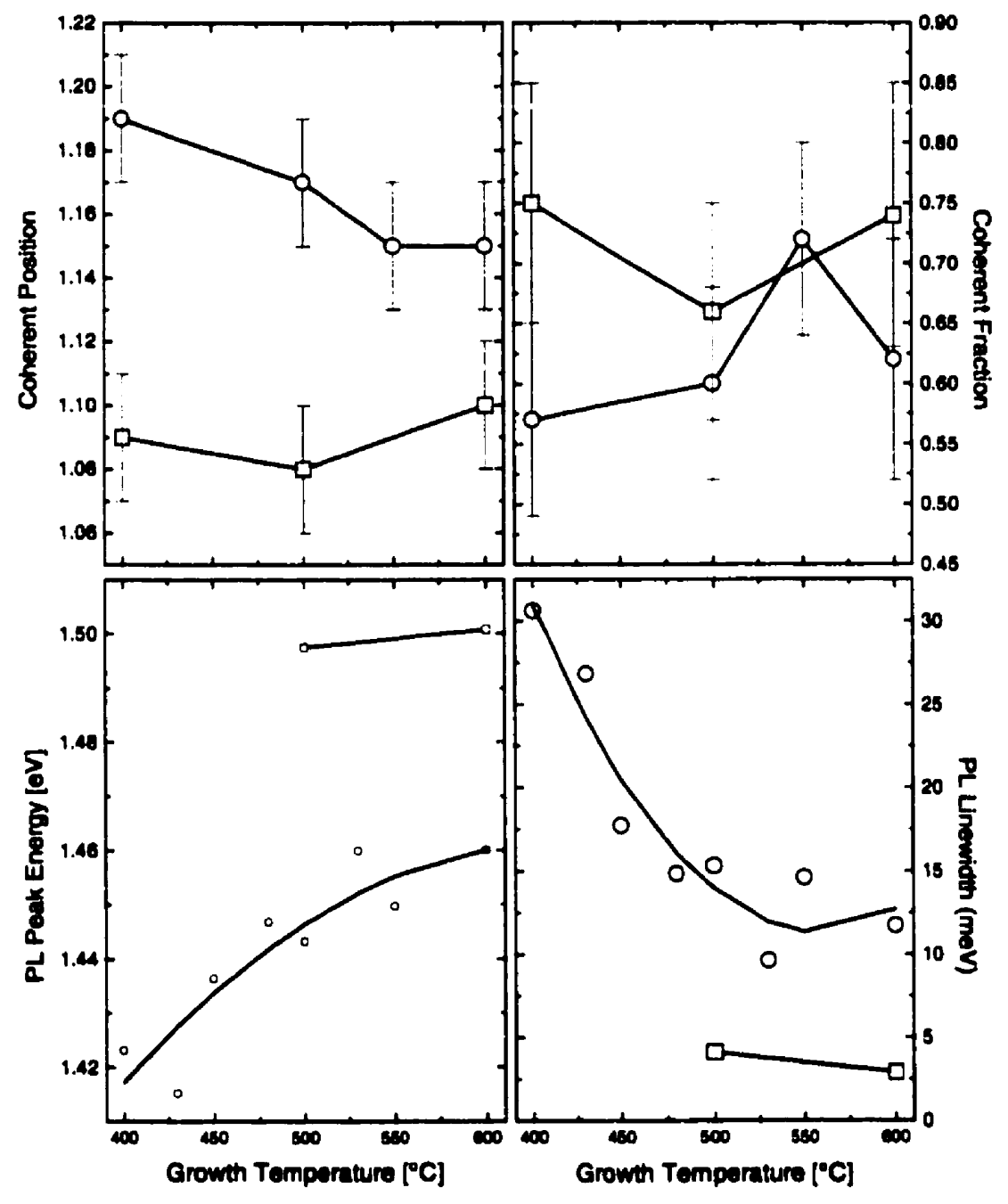

Figure 7.38: Summary of the XSW and PL results for the main group of MOVPE samples. The circles and squares correspond to $1 \mathrm{ML}$ and 1/2 ML samples, respectively. For the PL data, the uncertainties are given approximately by the symbol size. The solid lines are guides for the eye. 
incorporation in islands rather than in graded $\operatorname{In}_{x} \mathrm{Ga}_{1-r}$ As layers. If these islands have lateral extents greater than the exciton radius. then the net effect would be a decrease in the emission energy due to the fact that the exciton samples a potential well of thickness significantly greater than $1 \mathrm{ML}$, while maintaining an average laver thickness of approximately 1 ML $[5,6]$.

The lack of any clear trend for the 1/2 ML samples is noteworthy: since the MOVPE InAs growth exposures for these samples were half the corresponding full ML exposures. we might expect similarities between the two series. The lack thereof may be related to details of the growth mechanism at the subML scale. For example. the growth could take place in either 2-dimensional or 3-dimensional modes. or could involve compositional alloying, the details of which are difficult to resolve.

The final section of this chapter involves a study of both In-As and Cia-As bondlengths occuring in ternary alloy films grown in InP. This study will demonstrate the effects of both tensile and compressive strains in pseudomorphic epitaxy.

\subsection{Bond-length Distortions in InGaAs/InP(001) Heterostructures}

As mentioned in Section 5.1, ternary alloys of $\ln _{x} \mathrm{Ga}_{1-r}$ ts were grown by MOVPE in order to study the effect of strain on the bond-length distortions. This work was done in collaboration with Dr. Woicik at NIST. When $x=0.53$, the ternary lattice constant obtained from Vegard's law (Equation 5.3) is equal to the value for InP $\left(a_{I n P}=5.869 \AA\right)$. For an epitaxial film of this composition buried in $\operatorname{In} \mathrm{P}(001)$, there is no net strain and so the In-As and Ga-As bond lengths should have the same values as for a bulk alloy with this composition, i.e. in agreement with the results of Mikkelsen and Boyce [54]. By varying the composition it was possible to study this lattice-matched ( $\mathrm{LM})$ case. as well as the cases of tensile $(x<0.53)$ and compressive $(x>0.53)$ strain. In the limiting cases the strains are $\varepsilon_{\|}=+3.8 \%$ for $x=0$ and $\varepsilon_{\perp}=-$ $3.1 \%$ for $x=1$.

The samples were grown in a horizontal quartz reactor at 100 Torr and $560^{\circ} \mathrm{C}$. 
SUMITOMO InP(001) Fe-doped substrates were used with no additional cleaning. Each growth was preceded by the growth of an InP buffer layer $(\sim 800 \AA)$ using TMIn at $14.8 \mu \mathrm{mol} / \mathrm{min}$ and TBP at $409 \mu \mathrm{mol} / \mathrm{min}$. The TMIn flow was then reduced to the desired flow rate for the rest of the structure, and an additional, thin InP buffer layer was grown at the new flow rate. In order to form abrupt interfaces with minimal group $V$ mixing. the growth interruption scheme of Holmes $t$ al. was employed [141]. Following the InP buffer growth, the growth was interrupted first under TBP for $0.5 \mathrm{sec}$, and then under $\mathrm{H}_{2}$ for $0.5 \mathrm{sec}$. The $\ln _{r} \mathrm{Ga}_{1-x}$ As layer was then deposited by simultaneously flowing TEGa. TMIn and TBAs. Following the alloy growth another pair of interruptions of $0.5 \mathrm{sec}$ under $\mathrm{H}_{2}$, and $0.5 \mathrm{sec}$ under TBP was used. A thin InP cap layer was then deposited at the same TMIn flow rate as for the alloy layer. Holmes et al. found that the elimination of TBAs from the growth interruptions leads to a reduction in interface effects for the $\operatorname{InCaAs} / \operatorname{In} P$ interface. The alkyl flow rates are given in Table 7.9 . For all three samples a TBAs flow rate of $57.2 \mu \mathrm{mol} / \mathrm{min}$ was used to give a V/III ratio of 4.8 .

\begin{tabular}{|c|c|c|c|c|c|c|}
\hline \hline Sample & TMIn $(\mu \mathrm{mol} / \mathrm{min})$ & TEGa $(\mu \mathrm{mol} / \mathrm{min})$ & $x^{\circ}$ & $x$ & $t(\mathrm{~A})$ & $t_{\text {cup }}(\mathrm{A})$ \\
\hline Tensile & 4.02 & 7.80 & 0.34 & 0.21 & 6.5 & 8.3 \\
\hline LM & 7.91 & 3.97 & 0.67 & 0.50 & 1095 & 85 \\
\hline Compressive & 9.30 & 2.59 & 0.78 & 0.74 & 53 & 93 \\
\hline \hline
\end{tabular}

Table 7.9: Alkyl flow rates and input vapour phase composition. $x^{v}$. for MOVPE growth of InGaAs layers on InP. The solid phase composition, $x$, thickness, $t$, and cap thickness, $t_{\text {cap }}$ were determined by XRD.

The strained layer thicknesses and compositions were determined by HRXRD as shown in Figure 7.39 . To ensure coherency of the layers with the substrates, the strained layers were necessarily very thin. It was verified using AFM that layers of these thicknesses and composition were below the critical thicknesses for pseudomorphic growth.

In the HRXRD measurements, the slit shown in Figure 6.5 was replaced by a circular pinhole of approximately $2 \mathrm{~mm}$ diameter to collimate the beam and allow position-dependent measurements across each sample. The results for the LM sample 
are given in Table 7.9. From the HRXRD data obtained at several positions (Figure $\mathbf{i . 4 0}$ ) it was found that the composition is not laterally uniform. This is a problem intrinsic to this horizontal reactor which may be related to gas-phase depletion effects downstream. Insufficient data were obtained to quantify this spread in the strained samples and so the XRD results should not be considered mean values of $x, t$ or $t_{\text {cap }}$. A comparison between the In fraction in the input vapour and the measured values is shown in Figure 7.41 . The ratio $x / x^{v}$ is called the distribution coefficient [21].

The XAFS measurements were performed at beamline X23A2 by monitoring the Ga and As fluorescence yield as a function of incident photon energy and flux above both the $\mathrm{Ga}\left(E_{0}=1036 \mathrm{eV}\right)$ and $\mathrm{As}\left(E_{0}=1186 \mathrm{i} \mathrm{eV}\right) \mathrm{K}$-edges. The data presented here were obtained using either a Lytle detector or a single-element Si detector. A glancing-incidence geometry was used. although the alignment was much less rigorous than for the InAs SQW measurements, partly because of the lower edge energies involved. The critical angles are $0.23^{\circ}$ and $0.20^{\circ}$ at $200 \mathrm{el}$ above the $\mathrm{Ga}$ and $\mathrm{As}$ edges. respectively. Each $1 / 4$ wafer sample was adhered to an $\mathrm{Al}$ plate of thickness $\sim 2 \mathrm{~mm}$ using a melted wax layer. and then the plate was taped to a small RADIO SHACK computer fan. In order to avoid contamination to the spectra arising from Bragg peaks the samples were spun around their surface normals during data acquisition using the fan. The incidence angles were adjusted using a stack of washers mounted under the downstream end of the fan.

For this study the data were analyzed using empirical phase and amplitude standards obtained from powder samples in transmission. Powder data from GaAs. InAs and $\mathrm{GaP}$ standards were processed with AUTOBK using the methods described in Section 7.7 . As a reliability check the powder datasets were analyzed using FEFF $6 \mathrm{~b}$ models for the GaAs and GaP crystals at the Ga K-edge, and GaAs and InAs crystals at the As K-edge. The raw transmission data and the fits to the FFFS contributions are shown in Figures 7.42 and 7.43 .

These figures clearly show that, with the exception of the InAs powder measured at the As K-edge, the EXAFS data contain significant contributions from the first shells, as well as second and higher-order shells. This is also seen in the FTs shown in Figure i.44. In particular the GaP powder has a second shell of comparable amplitude 


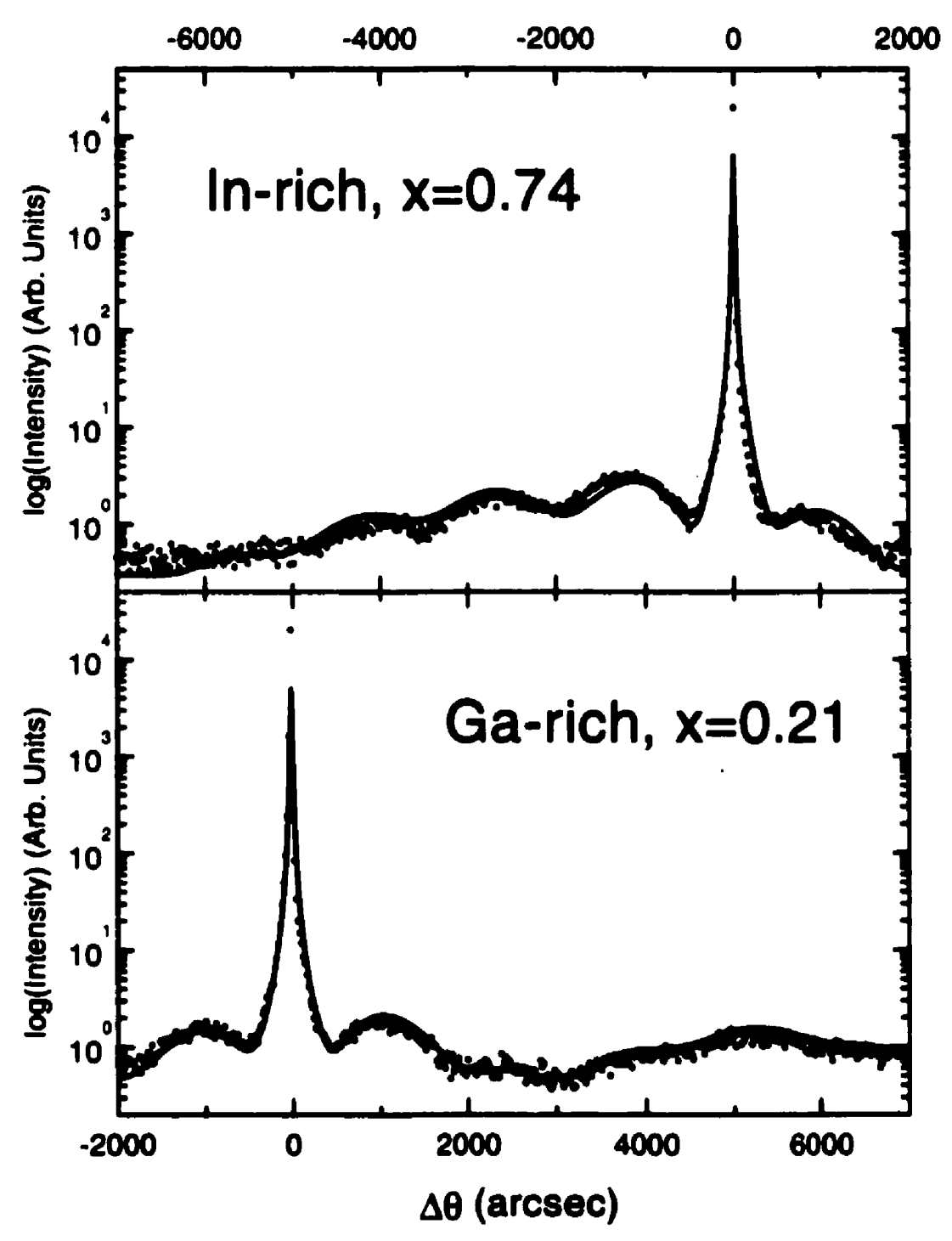

Figure 7.39: $\mathrm{X}$-Ray rocking curve data obtained near the $\ln \mathrm{P}(004)$ symmetric substrate reflection, for $\Delta \theta=\theta-\theta_{B}$. The data for the strained $\ln _{x} \mathrm{Ga}_{1_{-x}}$ As films are indicated by circles and the solid lines indicate the results of dynamical simulations using the $x$-values indicated and the layer thickesses as described in the text. 


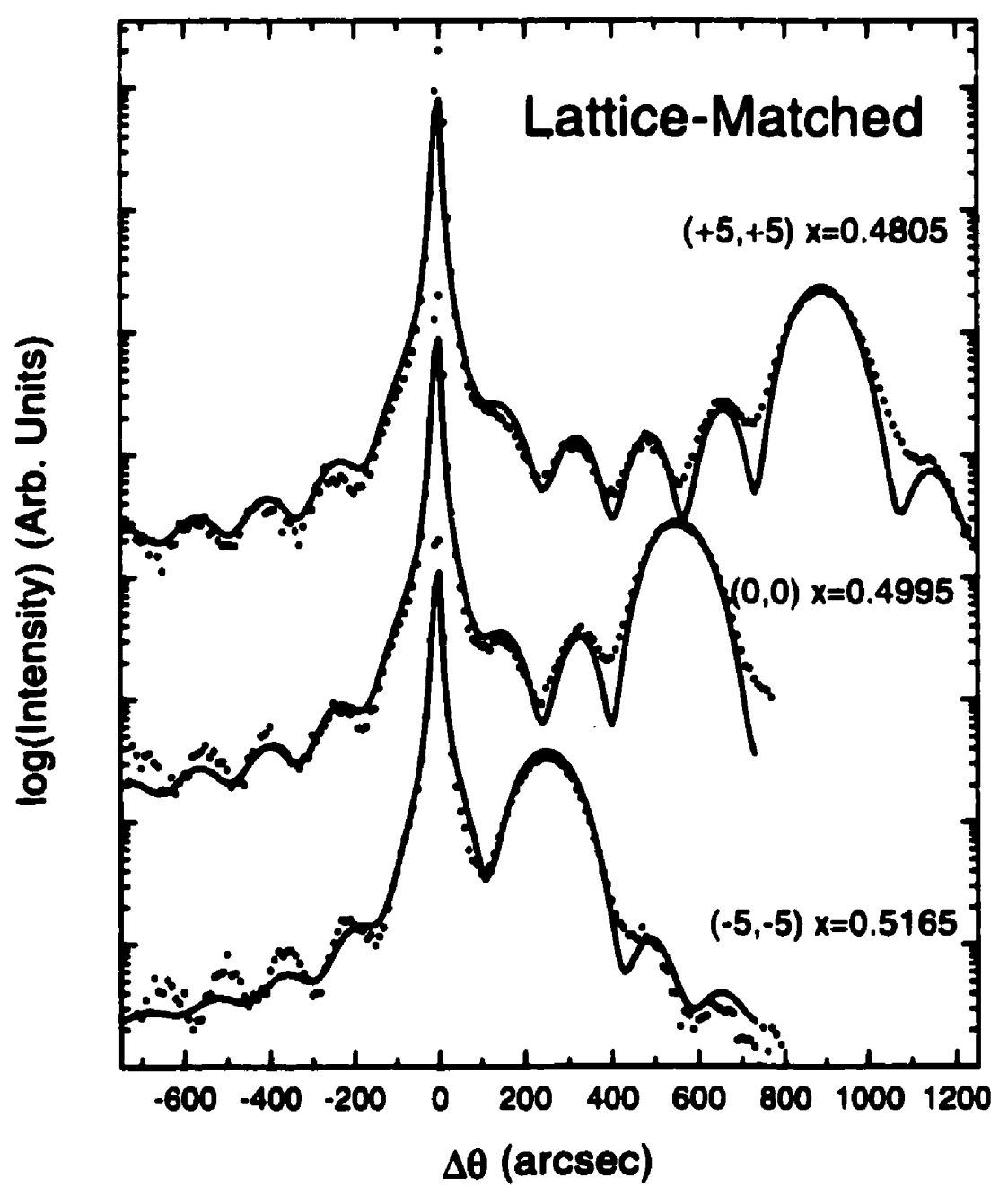

Figure 7.40: X-Ray rocking curve data obtained near the $\operatorname{InP}(004)$ symmetric substrate reflection. The data were obtained using pinhole-collimation at different positions on the nominally lattice-matched sample (the horizontal and vertical positions are given in $\mathrm{mm}$ ). The data are indicated by circles and the solid lines indicate the results of dynamical simulations using the $x$-values indicated and the layer thickesses as described in the text. 


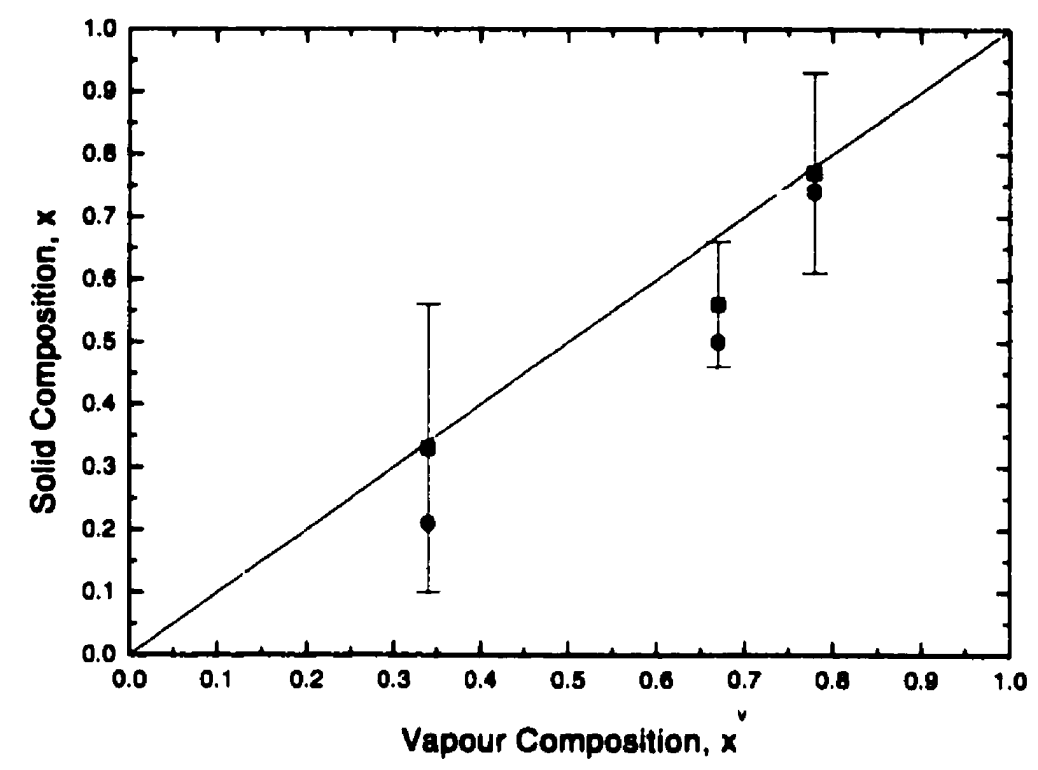

Figure 7.41 : Indium composition. $x^{v}$. in the input vapour phase compared with the composition determined by XRD (circles) and EXAFS (squares and error bars) measurements. The solid line corresponds to a distribution coefficient of unity.

to the first shell. It is interesting to note the close correspondence between the Ga and As $K$-edge FTs for the GaAs powder over the whole $R$-range shown in the figure. After Fourier-filtering the data were fitted with the FEFF phases and amplitudes, yielding the results shown in Table 7.10 . In the fits the variable parameters were $S_{0}^{2}, R, \sigma^{2}$ and $\Delta E_{0}$. The agreement with the known bond lengths is excellent, except for the Ga-P bond length, in which case the expected value is just outside the error bar. Note that the As K-edge data indicate that $S_{0}^{2}$ depends upon the chemical environment of the absorbing atom in agreement with the observations of $\mathrm{Li}$ et al. [140].

Figures $i .45$ and $i .46$ show the raw $k^{2}$-weighted $G a$ and As K-edge EXAFS data from the alloy layers, respectively. Comparing the data with the transmission data of Figures 7.42 and $\mathbf{7 . 4 3}$ it is clear that the alloy data consist mainly of first-shell 


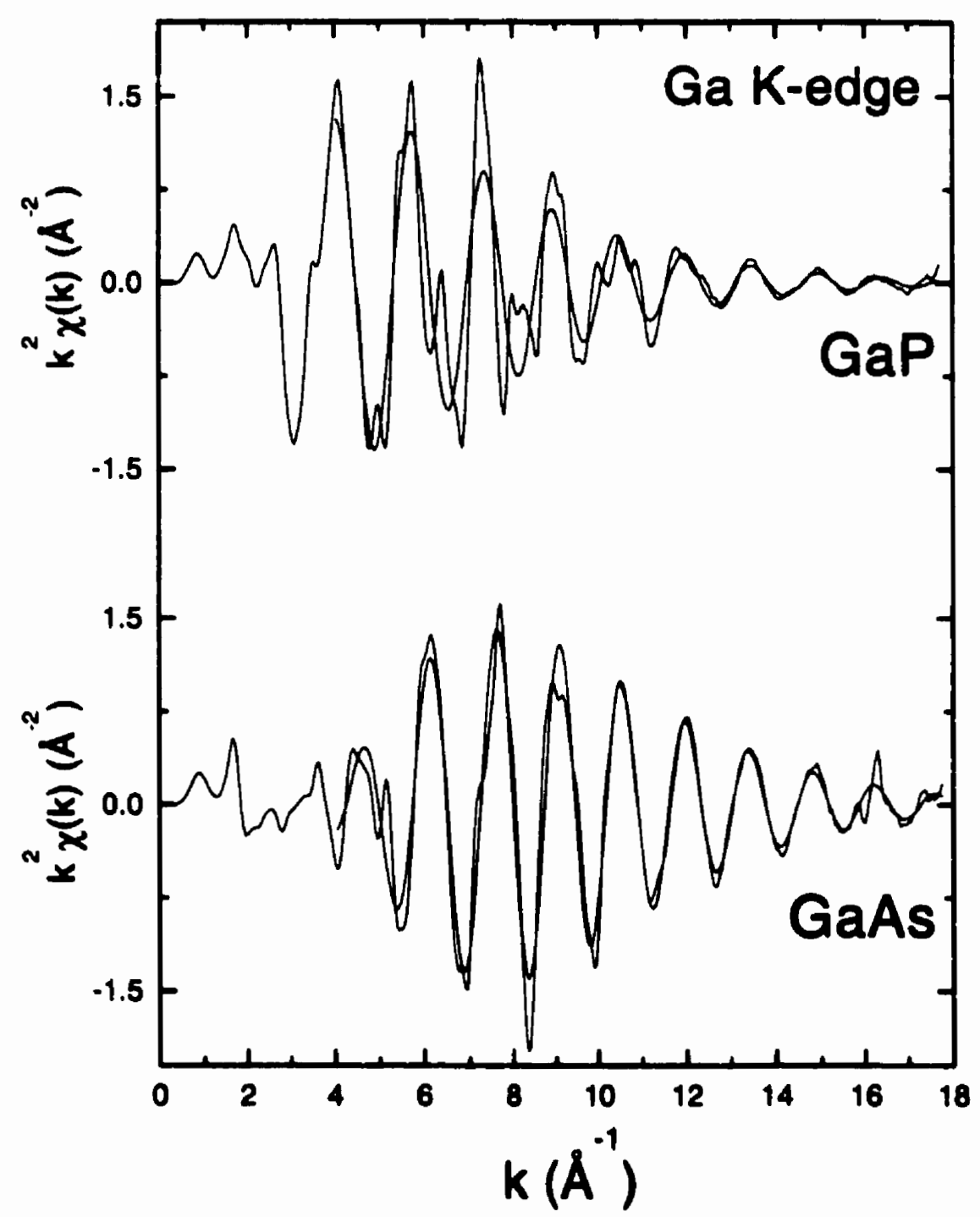

Figure 7.42: $k^{2}$-weighted Ga k-edge EXAFS, $k^{2} \chi(k)$, for the GaAs and GaP powder specimens. Superimposed on the data are the fits to the Fourier-filtered first shell contributions, corresponding to the $\mathrm{Ga}-\mathrm{As}$ and $\mathrm{Ga}-\mathrm{P}$ nearest-neighbour bonds. 


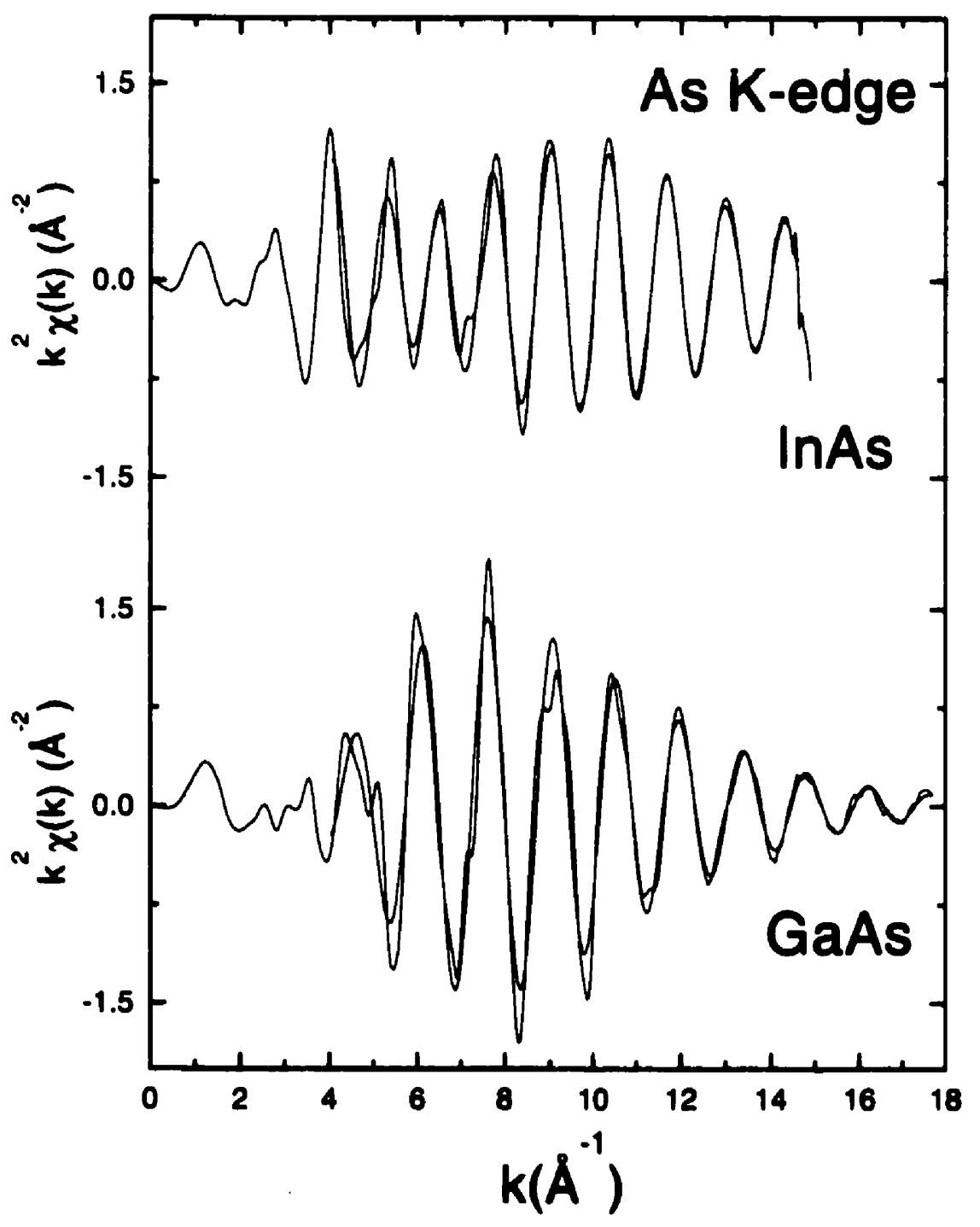

Figure 7.43: $k^{2}$-weighted As K-edge EXAFS, $k^{2} \chi(k)$, for the GaAs and InAs powder specimens. Superimposed on the data are the fits to the Fourier-filtered first shell contributions, corresponding to the As-Ga and As-In nearest-neighbour bonds. 


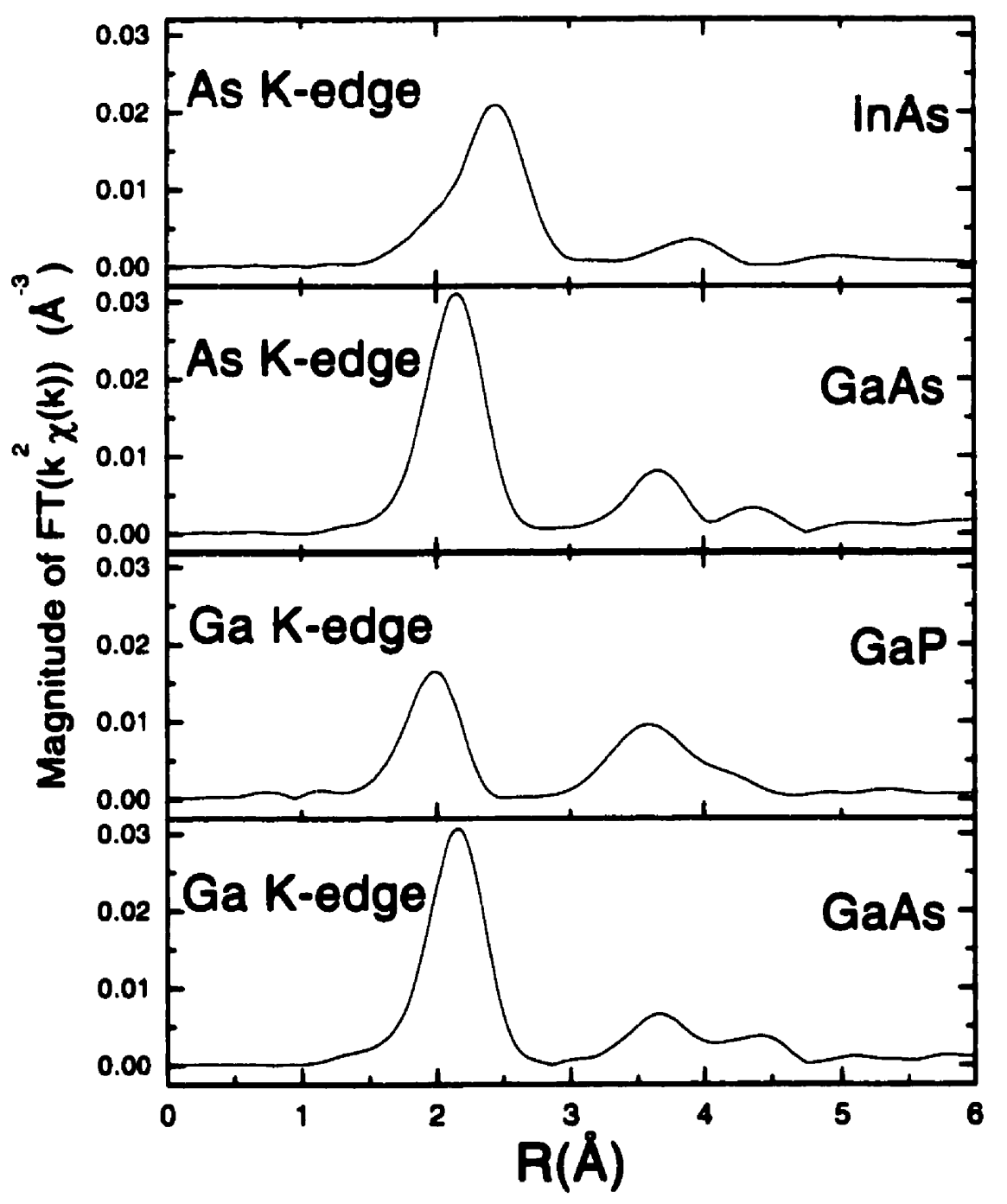

Figure 7.44: The magnitudes of the Fourier transforms of the $k^{2}$-weighted $\mathrm{Ga}$ and As K-edge EXAFS, $k^{2} \chi(k)$, from the reference powders. A $10 \%$ Gaussian window was used in each transform. The transform ranges (from top to bottom in the figure) were: $k=3.0 \rightarrow 14.6 \AA^{-1}, k=1.7 \rightarrow 17.7 \AA^{-1} . k=2.8 \rightarrow 17.7 \AA^{-1}$ and $k=1.9 \rightarrow 17.8 \AA^{-1}$. 


\begin{tabular}{|c|c|c|c|c|c|}
\hline \hline & & $S_{0}^{2}$ & $R(\AA)$ & $\sigma^{2}$ & $R_{0}(A)$ \\
\hline Ga K-edge & Ga-As & $1.03 \pm 0.07$ & $2.449 \pm 0.004$ & $0.0048 \pm 0.0004$ & 2.448 \\
\hline & Ga-P & $1.07 \pm 0.05$ & $2.365 \pm 0.004$ & $0.0051 \pm 0.0005$ & 2.360 \\
\hline As K-edge & As-Ga & $1.03 \pm 0.03$ & $2.450 \pm 0.002$ & $0.0047 \pm 0.0002$ & 2.448 \\
\hline & As-In & $0.80 \pm 0.08$ & $2.626 \pm 0.005$ & $0.0041 \pm 0.0005$ & 2.623 \\
\hline \hline
\end{tabular}

Table 7.10 : Experimental determination of the bond lengths in powder standards from fitting with FEFF6b models. The known bond lengths. $R_{0}$, are shown for comparison. The error bars indicate the values which double the squared residuals in each parameter when the other values were allowed to float.

contributions. Superimposed on the alloy EXAFS data are the fits to the FFFS contributions. At the Cia-edge. the first shell consists almost exclusively of Ga-As bonds. The strained samples. because they are thinner. also contain small contributions from Ga-P bonds at the interfaces. At the As-edge, however. the first shell contains both As-Cia and As-In bonds. The data were fitted using the appropriate phase, $\omega_{i}(k)$. and amplitude. $\left|f_{i}(k)\right|$. functions from the standards to a function of the form $\backslash(k)=\sum_{i=1}^{2} \backslash{ }_{i}(k)$, where the sum is taken over the first shell. In the fits only the coordination numbers. $N_{i}$, and the bond lengths, $R_{i}$, were varied. Table $i .11$ shows the results of the fitting. The alloy compositions. $x$. determined from the coordination results are shown in Figure 7.41.

\begin{tabular}{|c|c|c|c|c|c|c|}
\hline \hline$x$ & Ga K-edge & $N$ & $R(\mathrm{~A})$ & As K-edge & $N$ & $R(\mathrm{~A})$ \\
\hline 0.21 & Ga-As & $3.7 \pm 0.3$ & $2.477 \pm 0.004$ & As-Ga & $2.9 \pm 0.5$ & $2.475 \pm 0.010$ \\
\hline & Ga-P & $0.2 \pm 0.3$ & $2.357 \pm 0.25$ & As-In & $1.4 \pm 0.6$ & $2.618 \pm 0.021$ \\
\hline 0.50 & Ga-As & $4.1 \pm 0.4$ & $2.466 \pm 0.005$ & As-Ga & $1.9 \pm 0.2$ & $2.469 \pm 0.005$ \\
\hline & Ga-P & & & As-In & $2.4 \pm 0.2$ & $2.611 \pm 0.005$ \\
\hline 0.74 & Ga-As & $3.7 \pm 0.2$ & $2.463 \pm 0.003$ & As-Ga & $1.0 \pm 0.2$ & $2.465 \pm 0.010$ \\
\hline & Ga-P & $0.2 \pm 0.3$ & $2.36 \pm 0.09$ & As-In & $3.4 \pm 0.3$ & $2.607 \pm 0.003$ \\
\hline \hline
\end{tabular}

Table 7.11: Experimental determination of the bond lengths and coordination numbers in $\operatorname{In}_{x} \mathrm{Ga}_{1-x}$ As alloys on $\operatorname{In} \mathrm{P}(001)$. Independent results obtained at both the $\mathrm{Ga}$ and As K-edges are tabulated. The error bars indicate the values which double the squared residuals in each parameter when the other values were allowed to float.

The FTs of the EXAFS data for the ternary alloys are shown in Figure $i .4 \pi$. Comparing this figure with the FTs of the reference powders it is clear that the 


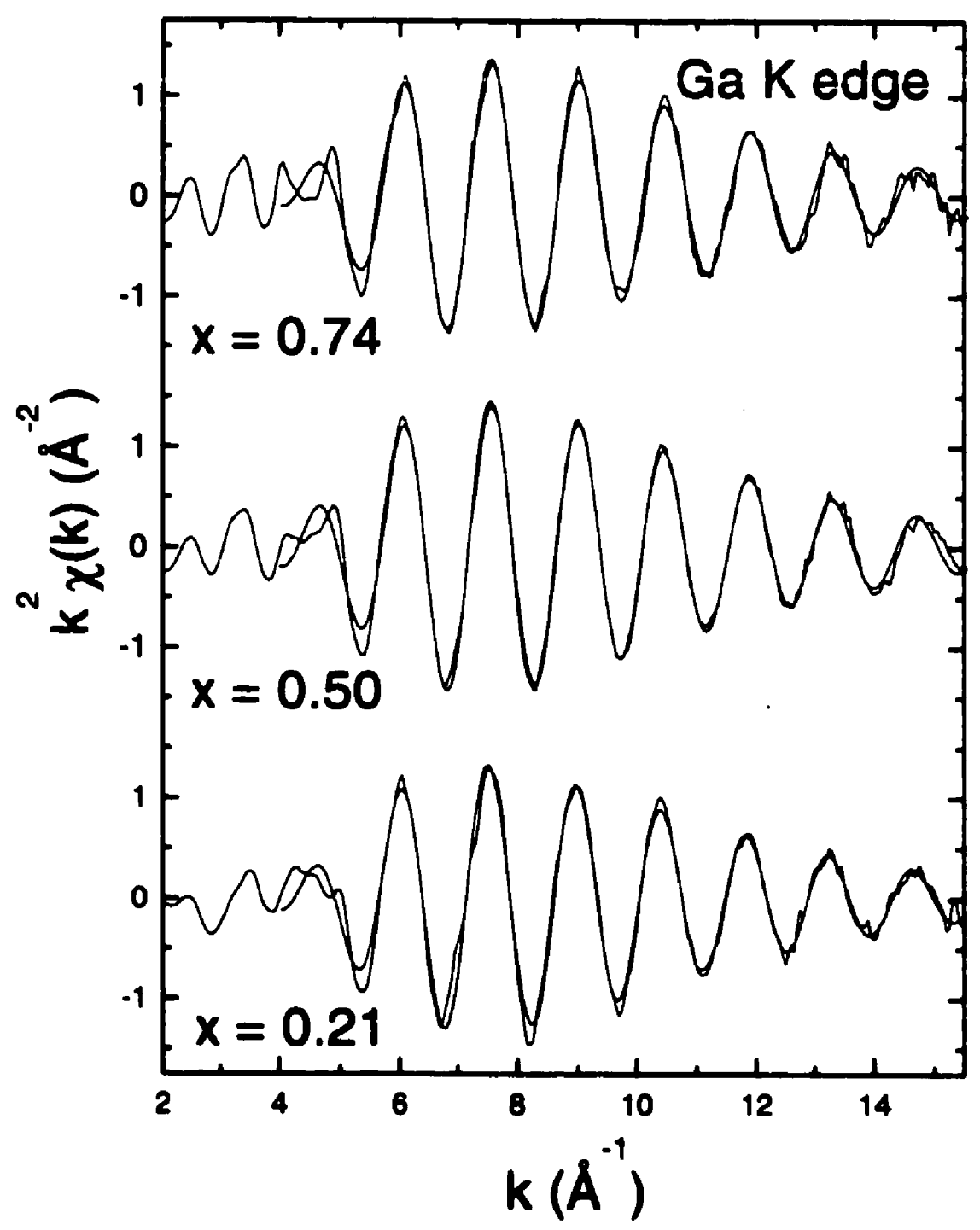

Figure 7.45: $k^{2}$-weighted Ga $k$-edge EXAFS, $k^{2} \gamma(k)$, from pseudomorphic $\operatorname{In}_{x} \mathrm{Ga}_{1-x}$ As alloys on $\operatorname{In} \mathrm{P}(001)$. Superimposed on the raw data are the fits to the Fourier-filtered first shell contributions consisting mainly of Ga-As bonds. 


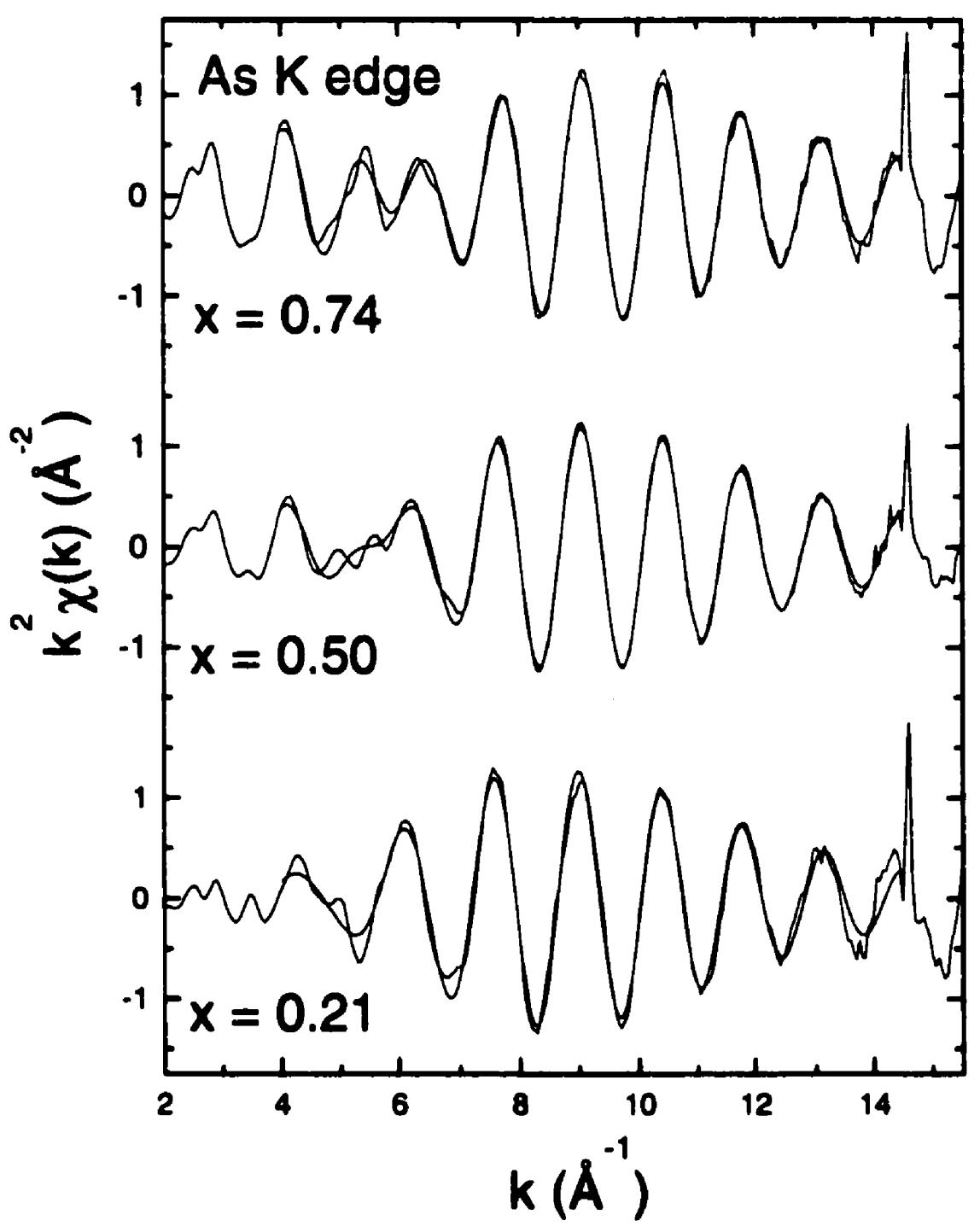

Figure 7.46: $k^{2}$-weighted As Ki-edge EXAFS, $k^{2} \chi(k)$, from pseudomorphic $\operatorname{In}_{x} \operatorname{Ca}_{1-x} A$ s alloys on $\operatorname{In} P(001)$. Superimposed on the raw data are the fits to the Fourier-filtered first shell contributions which contain both As-Ga and As-In bonds. 
second and higher shells are less well-defined. For the As K-edge data the first shell is less-well-defined than for the Ga edge. This is due to the fact that As absorbers are bonded to both $\mathrm{Ga}$ and In atoms, while $\mathrm{Ga}$ absorbers are bonded primarily to $\mathrm{As}$ atoms, with a small contribution due to Ga-P bonds.

To compare these results with theory, a random cluster calculation was performed following the formalism of Reference [62], which was briefly described in Section 5.1.3. In the present case the calculation was performed by relaxing the atoms within sixteenbond $\mathrm{In}_{j} \mathrm{C}_{a_{1-j}}$ As clusters $(j=0.1 .2 .3)$ embedded in virtual-crystal media. Figure $i .48$ shows the resulting In-As and Ga-As bond lengths calculated for cubic clusters and for clusters that have been tetragonally distorted for pseudomorphic growth on $\operatorname{In} P(001)$. The bulk bond lengths follow the measurements of Mikkelsen and Boyce [54] and clearly do not support the VCA. The tetragonally-distorted bond lengths closely follow the experimental data. As mentioned at the beginning of this section, the bulk and strained bond lengths are predicted to be equal for L.M films.

The downward slope of the strained-layer predictions clearly shows that the external strain imposed on the layer by the substrate opposes the natural bond-length distortions due to alloying. The tetragonally distorted In-As and Ga-As bond lengths are monotonically decreasing functions of composition. with a slight bowing due to the different $C_{12} / C_{11}$ values for InAs and GaAs, which gives a nonlinear dependence to $a_{\perp}$. As in the case of bulk bond lengths, the strained bond lengths follow nearly parallel curves despite their different lengths and force constants. This experiment demonstrates that the bond lengths in strained-layer semiconductors deviate significantly: from their bulk-alloy values, and the deviations are found to be uniform functions of the epitaxially-induced strain.

This concludes the presentation and the analysis of the experimental results obtained in this work. The next chapter will briefly summarize the results and suggest future directions. 


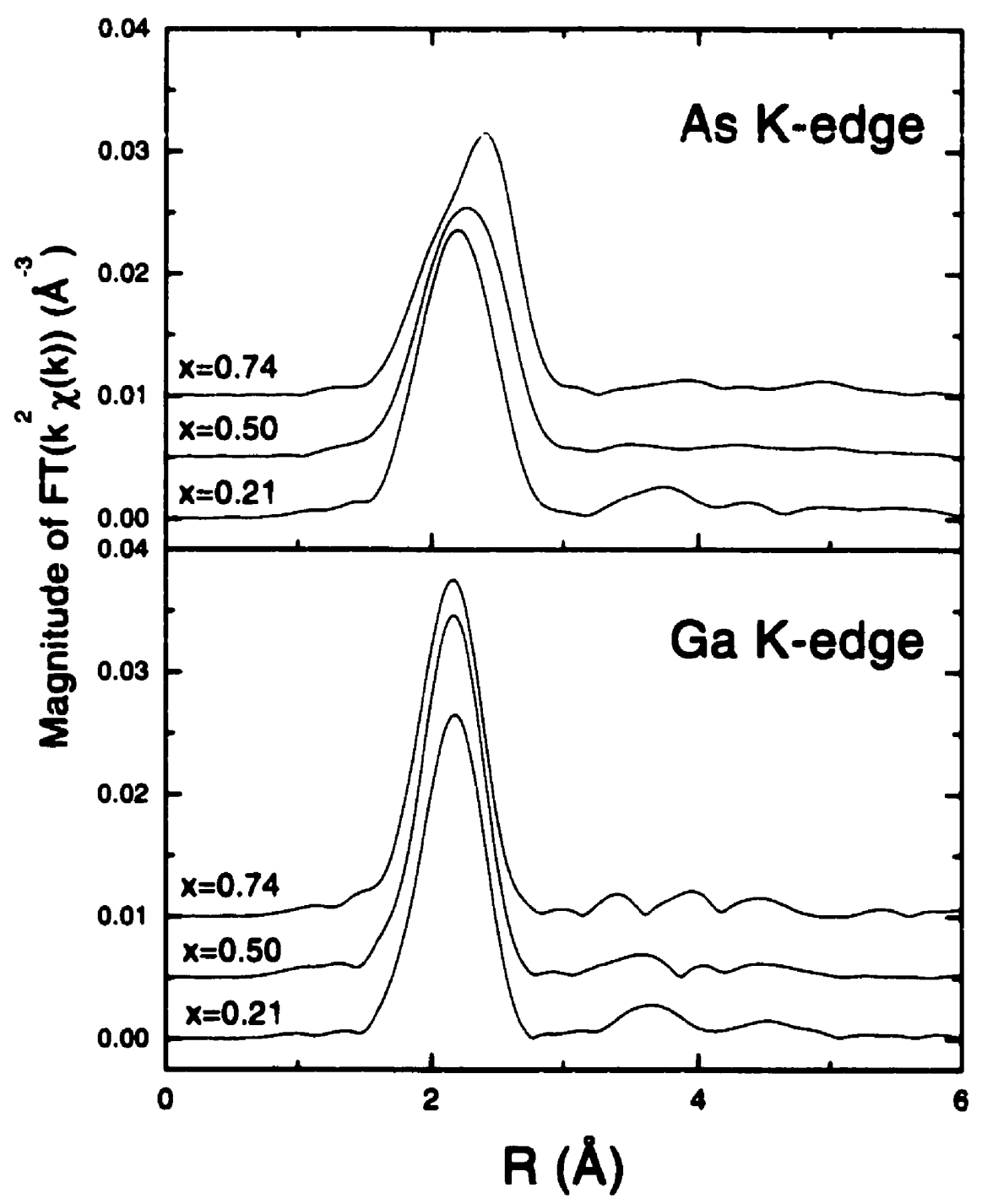

Figure $\bar{i} .4 \bar{i}$ : The magnitudes of the Fourier transforms of the $k^{2}$-weighted Ga and As Ki-edge EXAFS, $k^{2} \gamma(k)$, for the ternary alloys. A $10 \%$ Gaussian window was used in each transform. For the As-edge the transforms were taken over a range $k=2.3 \rightarrow 14.5 \AA^{-1}$. For the Ga-edge the transforms were taken over a range $k=2.3 \rightarrow 16.2 \AA^{-1}$, except for the sample with $x=0.21$, where the transform window began at $k=2.5 \AA^{-1}$. 


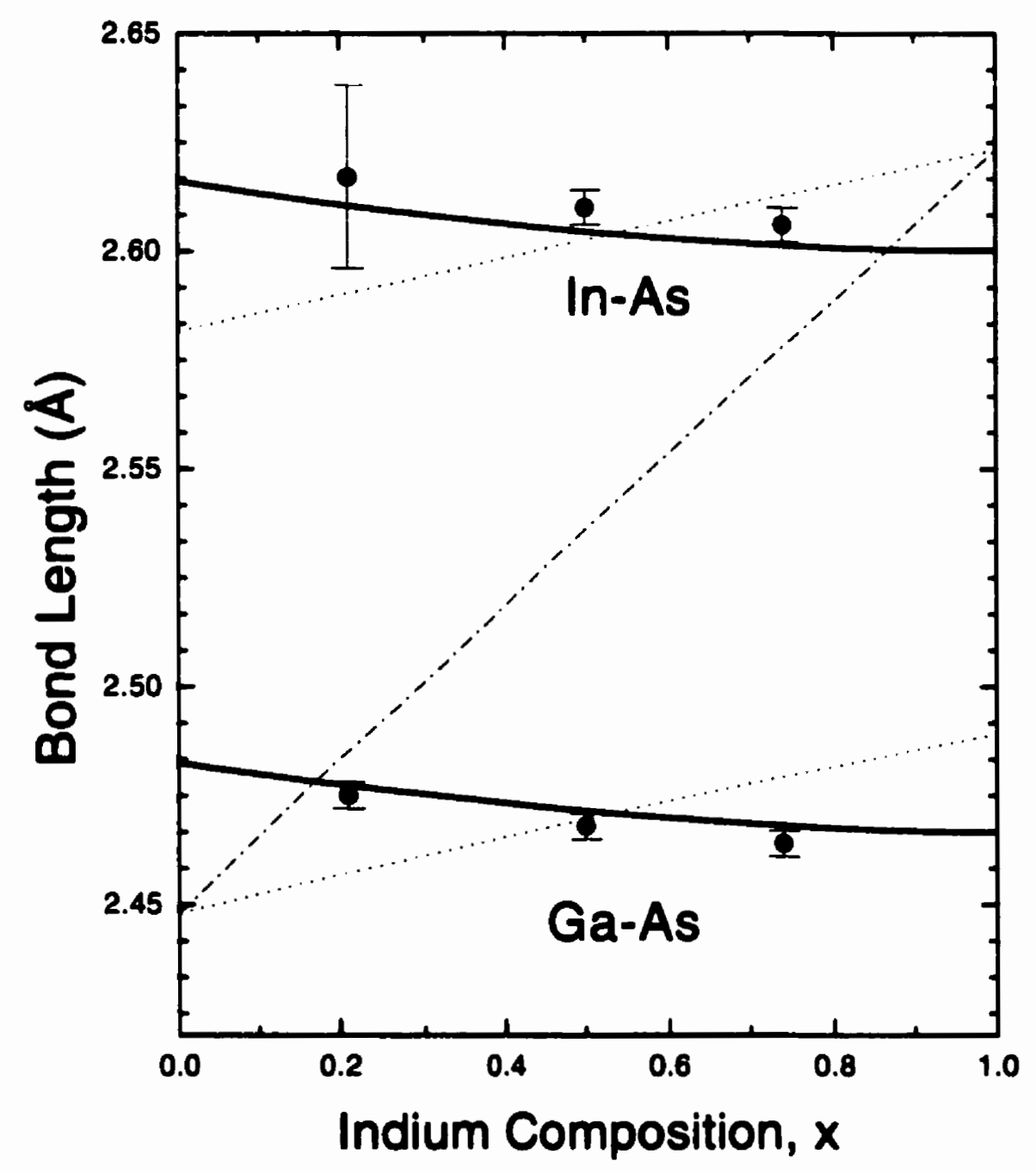

Figure 7.48: Comparison between the experimentally-determined In-As and Ga-As bond lengths in pseudomorphic $\operatorname{In}_{x} \mathrm{Ga}_{1-x}$ As alloys on $\operatorname{In} \mathrm{P}(001)$ and the results of the random cluster calculation. The dotted lines are the calculated cubic (bulk) bond lengths and the solid lines are the calculated tetragonal (strained) bond lengths. The dash-dot line is the bulk virtual crystal approximation prediction. Note that the bulk and strained bond lengths coincide for $x=0.53$, i.e. the lattice-matched, zero-strain condition. 


\section{Chapter 8}

\section{Conclusions}

This thesis has described the growth and characterization of ultrathin InAs SQW's in Ciats(001). Films 1 ML and 1/2. ML thick were grown by MOVPE and ALE and studied using a range of techniques, in which synchrotron measurements figured prominently.

XSW measurements of 1 and $1 / 2$.ML InAs MBE films revealed that the lavers do not contain complete In planes residing in well-defined positions. The XSW coherent positions were lower than expected for an ideally-strained ML and related to the composition, $x$, of single $\operatorname{In}_{x} \mathrm{Ca}_{1-s}$ As layers, with In compositions less than unity because of inadequate growth rates or interface roughness. Measurements in samples grown at various temperatures clarified the competing requirements for the growth of abrupt interfaces. Buffer layer growth temperatures should be sufficiently high to promote smooth surfaces. InAs layers and the first several GaAs cap layers should then be grown at lower temperatures to reduce In segregation.

Similar coherent positions were measured in ALE-grown films [142]. 1 and 1/2 ML films grown using an ALE sequence which produces good optical emission were found to consist of $\operatorname{In}_{x} \mathrm{Ga}_{1-x}$ As layers, with $x=0.79 \pm 0.07$ and $x=0.45 \pm 0.15$. respectively. In the subML case, this result suggests that the layer does not contain ML-high islands, but a uniform distribution of In atoms. In a sample grown with carefully controlled conditions to produce $1 \mathrm{ML} /$ cycle GaAs cap layers, the In incorporation was slightly higher, at $x=0.89 \pm 0.14$. A study of the RDS transients during the growth of GaAs cap 
layers showed that In segregation occurs to a large extent in ALE growth, regardless of the growth temperature or TMGa pulse duration. Models for the XSW parameters in the case of In segregation were unable to explain the low coherent positions measured in these films.

Growth of InAs SQWs in GaAs by conventional MOVPE was studied in-situ using multitransient RDS spectroscopy [143]. This method allowed the observation of the surface evolution from a CiaAs $d(4 \times 4)$-like spectrum under TBAs to the InAs (2×4)-like surface after 1 ML of InAs growth. Cap layer growth at various temperatures resulted in surface reconstructions characteristic of (ia.ts growth at those temperatures. No clear evidence of In segregation was observed with this technique.

'These SQW' structures were studied by HRXRD and synchrotron XSW [1.4] which showed that a growth temperature of $550^{\circ} \mathrm{C}$ produced an In.As layer exhibiting the best structural properties: a coherent position of $P^{H}=1.15 \pm 0.02$ and coherent fraction $F^{H}=0.72 \pm 0.08$. This excellent layer quality was achieved as a result of growth in a mass-transport-limited regime. high GaAs cap growth rate and low cation surface migration rates. At lower temperatures the higher coherent positions were interpreted using a segregation model involving a terrace incorporation of $I n$ atoms. Here the enhanced segregation arises because of surface kinetics: lower effective As-overpressure and lower growth rate.

PL spectroscopy revealed an increase in the observed emission energies with increasing growth temperatures, accompanied by a decrease in linewidth. The sharpest luminescence was observed in a sample grown at $530^{\circ} \mathrm{C}$, suggesting that a range 5330 $550{ }^{\circ} \mathrm{C}$ is optimal for producing the best $1 \mathrm{ML}$ films. At lower growth temperatures the lower emission energies arise because the islanded In distribution presents the excitons with a potential region greater than 1 ML thick.

In K-edge XAFS measurements were used to determine the In-As bond lengths using the polarization-dependent, glancing-incidence geometry. Bond lengths determined in MOVPE samples agree with the principle of strain accommodation through bond-bending, rather than bond stretching. No clear temperature-dependence was observed in these data, although they clearly showed that the samples grown at lower temperatures can not consist of single layers of In atoms, lending some credence to 
the model deduced from the XSW data. The second-shell contributions were unfortunately too small to contribute to the structural analysis. A verification of the analysis technique was achieved by fitting EXAFS data from an InAs standard, obtained in transmission. Curve-fitting based on a FEFF6b model was used to determine the In-As bond length to within $0.002 . A$ of the accepted value.

l'o further elucidate the mechanisms of strain accommodation in heteroepitaxy the In-As and $\mathrm{Ga}-\mathrm{As}$ bond lengths in a series of $\operatorname{In}_{x} \cdot \mathrm{Ga}_{1-r}$ As alloys of varying composition were determined using $\mathrm{Ga}$ and As K-edge EXAFS [68]. Fitting results using empirical amplitude and phase data agreed very well with a random cluster calculation. It was shown that the external strain imposed on the layer by the InP substrate opposes the natural bond-length distortions due to alloying.

A few directions for future research can be suggested at this time. Although the GaAs(004) substrate reflection was used for all XSW measurements presented here. other reflections can also be useful. To further clarify the In distribution in samples of this type it may be helpful to make XSW measurements using (111) or (22-4) reflections, for example. This is best accomplished by rocking the crystals through the Bragg angle at fixed photon energy and measuring the XSW in the usual way.

Other directions may involve completely different materials combinations. For instance, one could produce MOVPE SQW (or antiwell) structures containing several permutations and combinations of atoms from columns III and $V$ of the periodic table: AlAs in GaAs; GaAs in InAs: AlAs in InAs; or InAs in AlAs. It might be particularly interesting to study mixing on the group $V$-sublattice: GaSb in GaAs: InAs in InP, and so on. The results should follow the principles elucidated in this thesis, although the experiments are not at all trivial. In particular, one must carefully consider the emission energies of the constituent elements to ensure that the XSW signal from the desired species can be distinguished from the signal arising from other elements. The sample preparation also requires careful planning. For instance. AlAs and GaAs are so closely lattice-matched that a single AlAs layer embedded in Cia.As can not produce Pendellösung fringes in an HRXRD measurement. In this case one must apply creative methods to determine the amount of deposited Al. A very recent experiment by Lessmann, Brennan $e t$ al. used XSW to investigate $(\mathrm{AlAs})_{m}(\mathrm{GaAs})_{n}$ 
short-period SL structures through measurements at the 0th order SL satellite, as well as the substrate (004)-reflection [14.5].

Interesting EXAFS experiments can also be performed on technologically-relevant MOVPE-grown structures. For instance, issues of interface mixing in SL structures could be explored by performing EXAFS measurements at the various edges of the constituents. The DAFS technique is particularly promising for these applications. Measurements of fluorescence emission during glancing-incidence reflectivity scans (see Figure 7.28 ) can be used as a non-destructive tool for determining layer thicknesses and incorporated doses. This technique is particularly powerful in that it does not require crystalline material.

Finally, to build upon the sample preparation techniques yielding the favourable material quality and optical characteristics described in this thesis, it would be interesting to fabricate a CW laser diode employing an InAs SQW active region. Is mentioned. injection lasers of this type have yet to be demonstrated. MQW structures could be produced with different GaAs barrier thicknesses or different periodicity to tune the emission wavelength for desired applications. 


\section{Appendix A}

\section{Calculation of X-Ray Structure}

\section{Factors}

In this section the details of the structure factor calculations will be presented. This will include the definitions of the conventional unit cell for the zincblende lattice in real and reciprocal space. and the details of the atomic form factors. The latter makes use of the atomic scattering factors and the anomalous contributions $f^{\prime}$ and $f^{\prime \prime}$.

\section{A.1 Zincblende Structure}

The III- $V$ semiconductors studied in this work share a common structure: the zincblende (cubic zinc sulfide) structure, consisting of two interpenetrating $\mathrm{FCC}$ lattices with cation and anions occupying the lattice positions in each of these sublattices. Figure A.1 shows the atoms in a conventional unit cell. The atomic coordinates are $(0,0,0) ;\left(0, \frac{1}{2}, \frac{1}{2}\right) ;\left(\frac{1}{2}, 0, \frac{1}{2}\right) ;$ and $\left(\frac{1}{2}, \frac{1}{2}, 0\right)$ for one species, while atoms of the second species occupy the positions $\left(\frac{1}{4}, \frac{1}{4}, \frac{1}{4}\right) ;\left(\frac{1}{4}, \frac{3}{4}, \frac{3}{4}\right) ;\left(\frac{3}{4}, \frac{1}{4}, \frac{3}{4}\right)$ and $\left(\frac{3}{4}, \frac{3}{4}, \frac{1}{4}\right)$. These positions are defined relative to the lattice: $\vec{a}_{1}=(a, 0,0), \vec{a}_{2}=(0, a, 0)$ and $\vec{a}_{3}=(0,0, a)$ in real space. where $a$ is the lattice constant. In reciprocal space the unit cell is defined by the vectors $\vec{b}_{1}=\vec{a}_{1} / a^{2}$ and so on. ${ }^{1}$

\footnotetext{
${ }^{1}$ Factors of $2 \pi$ are not included, to be consistent with Batterman and Cole's notation which was used in Chapter 3.
} 


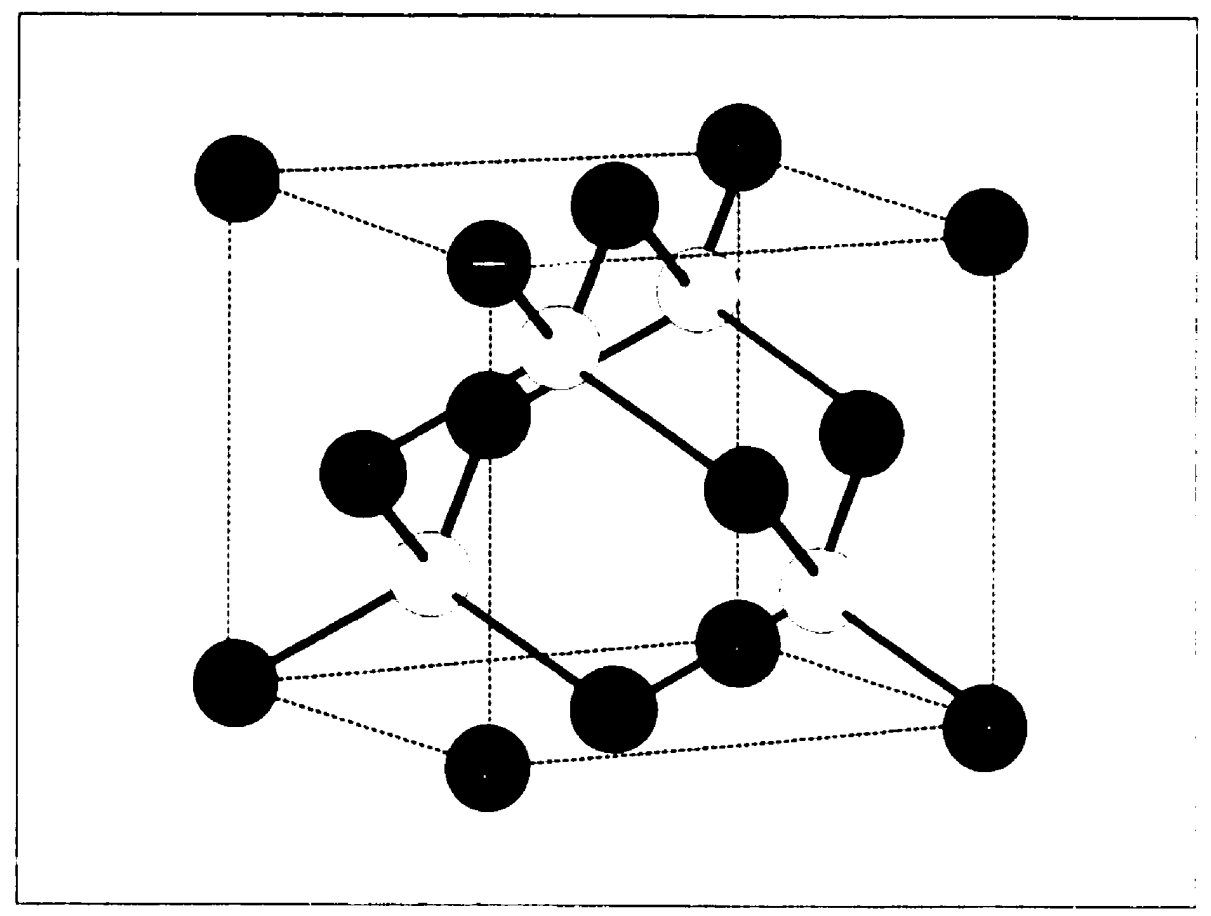

Figure A.1: The crystal structure of zincblende compound semiconductors.

The structure factor is written as a Fourier sum over the atoms in the unit cell:

$$
F_{H}=\sum_{n} f_{n} \exp \left(i 2 \pi \vec{H} \cdot \vec{r}_{n}\right) \text {. }
$$

where the reciprocal lattice vector for the Bragg reflection having Miller indices (HKL) is

$$
\vec{H}=H \vec{b}_{1}+K \vec{b}_{2}+L \vec{b}_{3} .
$$

Here $\vec{r}_{n}$ refers to the positions of atom $n$ within the real space unit cell, i.e.,

$$
\vec{r}_{n}=x_{n} \vec{a}_{1}+y_{n} \vec{a}_{2}+z_{n} \vec{a}_{3}
$$

The prefactor $f_{n}$ in Equation A.l is the atomic form factor of atom $n$. When the effects of absorption are included, this is a complex quantity and may be written

$$
f_{n} \equiv f_{n}^{0}+f_{n}^{\prime}+i f_{n}^{\prime \prime} \text {. }
$$


The first real component, $f_{n}^{0}$, describes the coherent scattering of X-Rays of wavelength $\lambda$ incident upon the sample at Bragg angle $\theta$. An analytic expression [146] may be used to describe the scattering from a given atom over a range of wavelengths and angles:

$$
f_{n}^{0}\left(\frac{\sin \theta}{\lambda}\right)=\sum_{i=1}^{4} a_{i} \exp \left[-b_{i}\left(\frac{\sin \theta}{\lambda}\right)^{2}\right]+c .
$$

The coefficients in the expansion for elements of interest are listed in Table A.I.

\begin{tabular}{|c|c|c|c|c|c|c|c|c|c|}
\hline & $a_{1}$ & $b_{1}$ & $a_{2}$ & $b_{2}$ & $a_{3}$ & $b_{3}$ & $a_{.1}$ & $b_{4}$ & $c$ \\
\hline Ga & 15.2354 & 3.06690 & 6.70060 & 0.241200 & 4.35910 & 10.7805 & 2.96230 & 61.4135 & 1.71890 \\
\hline As & 16.6723 & 2.63450 & 6.07010 & 0.264700 & 3.43130 & 12.9479 & 4.27790 & 47.7972 & 2.53100 \\
\hline In & 19.1624 & 0.547600 & 18.5596 & 6.37760 & 4.29480 & 25.8499 & 2.03960 & 92.8029 & 4.93910 \\
\hline \hline
\end{tabular}

Table A.1: Coefficients for analytical approximation to the atomic scattering factors for relevant elements.

The second real quantity. $f_{n}^{\prime}$, and the imaginary part. $f_{n}^{\prime \prime}$. are known as the anomalous scattering factors and account for resonance and absorption, respectively. For a given element these quantities may be found in the classic paper of Cromer and Liberman $[1+i]$ for common $X$-ray tube wavelengths. The tables contain the results of relativistic calculations of the photoelectric cross-sections across the periodic table. Extensive tables of $f^{\prime}$ and $f^{\prime \prime}$ have also been calculated over larger energy ranges using the method of Brennan and Cowan [148]. It is interesting to note that the photoabsorption cross-section for an element may be written

$$
\mu_{0}=2 r_{\mathrm{e}} \lambda f^{\prime \prime} \text {. }
$$

Figure A.2 shows the real and imaginary parts of the atomic form factors for the atoms relevant to both the diffraction and XAFS studies in this thesis. Note that the background absorption in XAFS measurements is related to the curves in the top of the figure. For a crystalline material, the mass absorption is given by

$$
\mu_{0}=\left(\frac{2 \pi}{\lambda}\right) \Gamma F_{0}^{\prime \prime},
$$

where the $(000)$-structure factor is written in terms of its real and imaginary parts as $F_{0}=F_{0}^{\prime}+i F_{0}^{\prime \prime}$. 


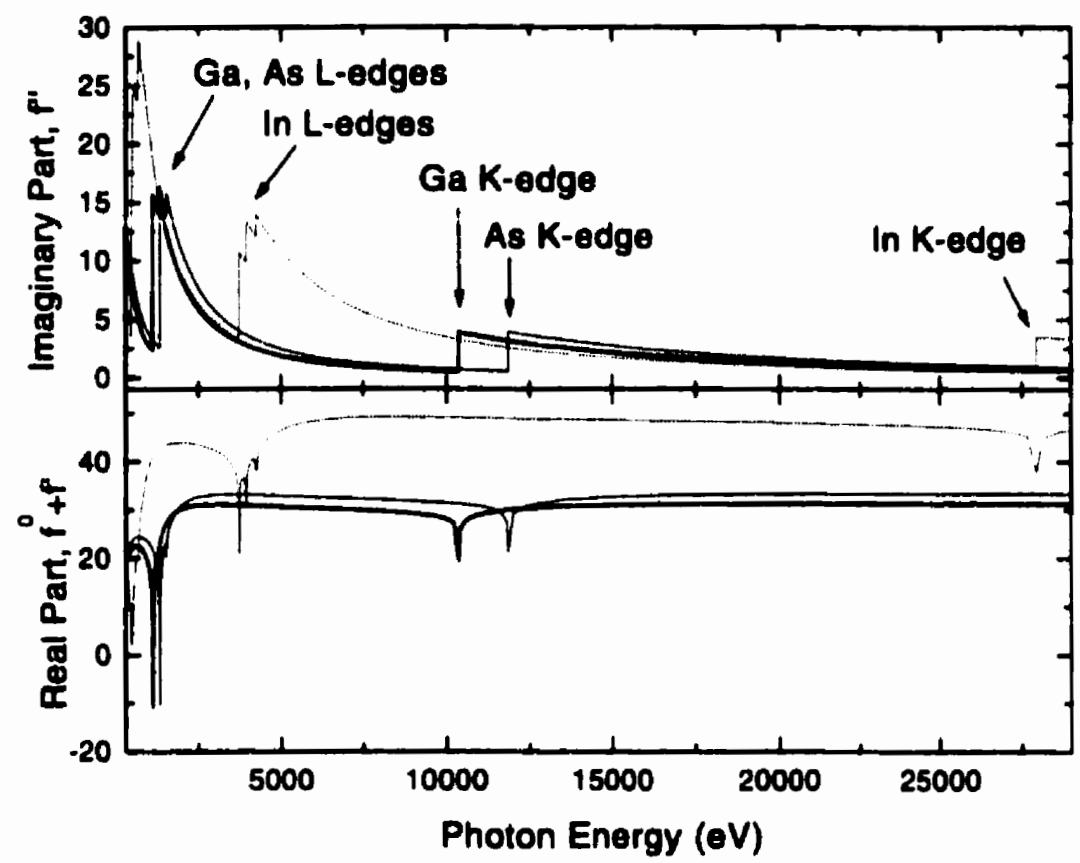

Figure A.2: The real and imaginary parts of the atomic form factors for Ga (heavy. solid lines), As (light. solid lines). and In (dashed lines). Abrupt changes are observed near the absorption edges, as indicated.

As an example, the structure factor for the GaAs (004)-reflection is calculated by taking the sum in Equation $\mathrm{A} .1$ over the $4 \mathrm{Ga}$ atoms and $4 \mathrm{As}$ atoms in the unit cell. One obtains

$$
F_{H}(004)=4\left[\left(f_{G a}^{0}+f_{G a}^{\prime}+i f_{G a}^{\prime \prime}\right)+\left(f_{A s}^{0}+f_{A s}^{\prime}+i f_{A s}^{\prime \prime}\right)\right] .
$$

Calculations of this type were used in the XSW data analysis described in Chapter 3.2.2. Thermal effects may be included by multiplying $F_{H}$ by the Debye-Waller factor for the reflection, $e^{-M}$ (see Section 3.2.2). 


\section{Bibliography}

[1] C. Giannini. L. Tapfer. S. Lagomarsino. J.C. Bouillard. A. Taccoen, B. Capelle. M. Ilg. O. Brandt and K.H. Ploog. Phys. Rev. B 48, 1 l196 (1993).

[2] J.C. Woicik. J.G. Pellegrino. S.H. Southworth. P.S. Shaw. B.A. Karlin. C.E. Bouldin and K.E. Miyano. Phys. Rev. B 52. R2281 (1995).

[3] J.C. Woicik. K.E. Miyano. J.G. Pellegrino. P.S. Shaw. S.H. Southworth and B.A. Karlin. Appl. Phys. Lett. 68, 3010 (1996).

[4] T.-L. Lee. Y. Qian. P.F. Lyman. J.C. Woicik. J.G. Pellegrino and M.J. Bedzyk. Physica B 221 (1996) 437.

[5] C.A. Tran, R.A. Arès. V.A. Karasyuk. S.P. Watkins, G. Letourneau and R. Leonelli. Phys. Rev. B 55, 4633 (199i).

[6] R.C. Iotti. L.C. Andreani and M. Di Ventra, Phys. Rec. B 57, R15072 (1998).

[i] A.R. Gonii, M. Stroh. C. Thomsen, F. Heinrichsdorff, V. Türck. A. Krost and D. Bimberg. Appl. Phys. Lett. 72, 1433 (1998).

[8] C. Guille. F. Houzay, J.M. Moison and F. Barthe, Surf. Sci. 189/190, 1041 (198i).

[9] J.M. Moison. C. Guille. F. Houzay, F. Barthe and M. Van Rompay, Phys. Ret. $B$ 40, 6149 (1989).

[10] O. Brandt, L. Tapfer, R. Cingolani, K. Ploog, M. Hohenstein and F. Phillipp. Phys. Rev. B 41, 12599 (1990). 
[11] J.H. Lee. K.Y. Hsieh and R.M. Kolbas, Phys. Rev. B 41, i678 (1990).

[12] M.L. Dotor, J. Meléndez, P. Huertas. A. Mazuelas. M. Garriga. D. Golmayo and F. Briones. J. Cryst. Cirowth 127, 46 (1993).

[1:3] M.I. Alonso, M. Ilg and K.H. Ploog. Phys. Rev. B 50. 1628 (1994).

[14] O. Brandt, L. Tapfer. K. Ploog. R. Bierwolf. M. Hohenstein. F. Phillipp. H. Lage and A. Heberle, Phys. Rev. B 44, 8043 (1991).

[15] N.N. Ledentsov. P.D. Wang. C.M. Sotomayor Torres. A. Yu. Egorov. M.V. Maximov. V.M. Ustinov. A.E. Zhukov and P.S. Lopev, Phys. Rev. B 50. 12171 (1994).

[16] R.A. Arès. Ph.D. Thesis. Department of Physics. Simon Fraser L'niversity (1997).

[17] W.J. Bartels. J. Hornstra and D.J.W. Lobeek. Actı ('ryst. A42. 5339 (1986).

[18] O. Brandt. K. Ploog. R. Bierwolf and M. Hohenstein. Phys, Rev. Lett. 68. 13339 (1992).

[19] H.M. Manasevit, .tppl. Phys. Lett. 116. 1725) (1969): H.M. Manasevit and W.I. Simpson. J. Electrochem Soc. 12, 156 (1968).

[20] C.A. Wang, S.H. Groves, S.C. Palmateer, D.W. Weyburne and R.A. Brown, J. Cryst. Growth 77, 136 (1986).

[21] G.B. Stringfellow, Organometallic Vapor-Phase Epitary - Theory and Practice. Academic Press, San Diego (1989).

[22] H. Watanabe and A. Usai, Inst. Phys. Conf. Ser. 83. 129 (1987).

[23] B.W. Batterman and H. Cole, Rev. Mod. Phys. 36. 681 (1964).

[24] A. Authier in X-Ray and Neutron Dynamical Diffraction - Theory and Applications, A. Authier, S. Lagomarsino and B.K. Tanner (eds.), Plenum Press, New York and London (1996). 
[25] S. Takagi. Acta Cryst. 15, 1311 (1962); J. Phys. Soc. Jap. 26, 1239 (1969).

[26] D. Taupin, Bull. Soc. Franc. Miner. Crist. 87, 469 (1964).

[2T] M. von Laue, Ergeb. Exakt. Naturw. 10. 1333 (1931).

[28] C.G. Darwin, Phil. Mag. 27. 315 (1914); ibid. 27. 675 (1911).

[29] J.A. Prins. Z. Phys. 63. 477 (1930).

[30] B.W. Batterman. Phys. Rev. Lett. 22. 703 (1969).

[31] J. Zegenhagen. Surf. Sci. Rep. 18. 199 (1993).

[32] N. Hertel. G. Materlik and J. Zegenhagen. Z. Phys. B 58, 199 (1985).

[33] D.P. Woodruff. D.L. Seymour. C.F. McConville. C.E. Riley. M.D. Crapper. N.P. Prince and R.G. Jones, Surf. Sci. 195. 23i (1988).

[34] R. Saravanan. S.K. Mohanlal and K.S. Chandrasekaran. Actu C'rystallogr. A 48. 4 (1992).

[35] J. Burgeat and D. Taupin, Acta Cryst. A24. 99 (1968).

[36] M.A.G. Halliwell, M.H. Lyons and M.J. Hill. J. Cryst. Cirowth 68, 523 (1984).

[3i] M.J. Hill. B.K. Tanner, M.A.G. Halliwell and M.H. Lyons, J. Appl. Cryst. 18. $446(1985)$.

[38] X-Ray Absorption, Principles, Applications, Techniques of EXAFS, SEIAFS and X.ANES. D.C. Koningsberger and R.Prins (eds.). John Wiley \& Sons, New York (1988).

[39] E.D. Crozier, Nucl. Instr. and Meth. in Phys. Res. B 133. 134 (199i).

[40] E.A. Stern in X-Ray Absorption. Principles, Applications, Techniques of EXAFS, SEXAFS and XANES, D.C. Koningsberger and R.Prins (eds.), John Wiley \& Sons, New York (1988). 
[41] J.J. Sakurai, San Fu Tuan (ed.) Modern Quantum Mechanics Rev. Ed., AddisonWesley, Reading MA (1994).

[42] D.E. Sayers. E.A. Stern and F.W. Lytle, Phys. Rev. Lett. 27, 1204 (1971).

[43] Woicik et al. have done much work on the SiGe binary alloy. In this case there is no true reference compound containing Si absorbers and Ge backscatterers or vice-versa. For an alternative empirical approach see: J.C. Woicik. K.E. Miyano. C.A. King, R.W. Johnson, J.G. Pellegrino. T.-L. Lee and Z.H. Lu. Phys. Ree. B 57. 14592 (1998).

[44] S.I. Zabinsky. J.J. Rehr. A. Ankudinov. R.C. Albers and M.J. Eller. Phys. Rev. B 52. 2995 (1995).

[45] D. Jiang. Ph.D. Thesis, Department of Physics, Simon Fraser University (1992).

[46] L.G. Parratt. Phys. Rec: 95, 359 (19.5-1).

[4i] V.G. Kohn. Phys. Stat. Sol. B 187. 61 (1995).

[48] A. Krost, G. Bauer and J. Woitok. in Optical Characterization of Epitaxial Semiconductor Layers. G. Bauer. W. Richter (eds.), Springer. Berlin (1996).

[49] M.J. Bedzyk, G.M. Bommarito and J.S. Schildkraut. Phys. Rev. Lett. 62. 1376 (1989).

[50] See. for example, I.V. Markov, Crystal Growth for Beginners, World Scientific. Singapore (1995).

[51] L.D. Landau and E.M. Lifshitz, Theory of Elasticity. Pergamon Press. Oxford (1970).

[52] J. Hornstra and W.J. Bartels, J. Cryst. Growth 44, 513 (1978).

[53] O. Madelung (ed.), Semiconductors: Group IV Elements and III-V Compounds. Springer, Berlin (1991). 
[54] J.C. Mikkelsen. Jr. and J.B. Boyce, Phys. Rev. Lett. 49, 1412 (1982).

[55] L. Tapfer and K. Ploog, Phys. Rev. B 33. 5565 (1986).

[56] L. Tapfer and K. Ploog, Phys. Ret. B 40. 9802 (1989).

[57] H. Holloway, J. Appl. Phys. 67. 6229 (1990).

[58] C. Shufan and M. Zhenhong. J. Appl. ('ryst 23. 14 ( (1990).

[59] K. Shiraishi and E. Yamaguchi. Phys. Rev. B 42, 3064 (1990).

[60] J. Massies and N. Grandjean. Phys. Rec. Lett. 71. 1.111 (1993).

[61] J.E. Bernard and A. Zunger. Appl. Phys. Lett. 65. 165 (1994).

[62] J.C. Woicik. Phys. Rev. B 57.6266 (1998).

[63] D. Rose. U. Pietsch. V. Gottschalch and H. Rhan. J. Phys. D: Appl. Phys. 28. A.246 (1995).

[6.t] C. Bocchi and C. Ferrari. J. Phys. D: Appl. Phys. 28. A164 (1995).

[65] W.A. Harrison, Electronic Structure and the Properties of Solids. Freeman. San Francisco (1980).

[66] J.C. Woicik, J.G. Pellegrino, B. Steiner. K.E. Miyano, S.G. Bompadre, L.B. Sorensen, T.-L. Lee and S. Khalid, Phys. Rev. Lett. 79, 5026 (1997).

[6i] J.C. Woicik. J.O. Cross. C.E. Bouldin. B. Ravel, J.G. Pellegrino. B. Steiner, S.G. Bompadre, L.B. Sorensen. K.E. Miyano and J.P. Kirkland. Phys. Rev. B 58, R4215 (1998).

[68] J.C. Woicik, J.A. Gupta. S.P. Watkins and E.D. Crozier, Appl. Phys. Lett 73. 1269 (1998).

[69] Y. Zheng, J.C. Boulliard, B. Capelle and C. Malgrange, Acta. Cryst. A54. 74 (1998). 
[70] Y. Zheng, J.C. Boulliard. B. Capelle, A. Lifchitz and S. Lagomarsino, Europhys. Lett. 41.623 (1998).

[i1] O. Brandt, K. Ploog, L. Tapfer, M. Hohenstein, R. Bierwolf and F. Phillipp. Phys. Rev. B 45, 8443 (1992).

[i2] G.A. Chadwick and D.A. Smith (eds.) Grain Boundary Structure and Properties. Academic. London (1975).

[i3] Z.Q. He, L. Ilver. J. Kanski. P.O. Nilsson and C.O. Karlsson. Phys. Rev. B 55. $9716(1997)$.

[i4] Y. Limura. K. Nagata. Y. Aoyagi and S. Namba. J. C'ryst. Crowth 105. 230 (1990).

[75] H. Yarnaguchi and Y. Horikoshi, J. Appl. Phys. 68, 1610 (1990).

[76] J. M. Moison, F. Houzay. F. Barthe, J.M. Gérard. B. Jusserand. J. Massies and F.S. Turco-Sandroff. J. C'ryst. Growth 111, 141 (1991).

[ii] J.M. Gérard and J.Y. Marzin, Phys. Ret. B 45, 6313 (1992).

[is] H.S. Hansen. A. Bensaoula. S. Tougaard. J. Zborowski and A. Ignatiev, J. Cryst. Growth 116. 271 (1992).

[79] M. Sato and Y. Horikoshi Surf. Sci. 267, 195) (1992).

[80] K. Muraki, S. Fukatsu, Y. Shiraki and R. Ito Appl. Phys. Lett. 61, 557 (1992); J. Cryst. Growth 127, 546 (1993).

[81] T. Kawai, H. Yonezu,.Y. Ogasawara, D. Saito and K. Pak Materials Research Society Proceedings 312. 113 (1993).

[82] J. Nagle, J.P. Landesman, M. Larive, C. Mottet and P. Bois. J. Cryst. Grouth 127, 550 (1993).

[83] M. Larive, J. Nagle, J.P. Landesman, X. Marcadet, C. Mottet and P. Bois, J. Vac. Sci. Technol. B 11, 1413 (1993). 
[84] A. Bosacchi, F. Colonna, S. Franchi, P. Pascarella. P. Allegri and V. Avanzini. J. Cryst. Growth 150, 185 (1995).

[85] O. Dehaese, X. Wallart and F. Mollot. Appl. Phys. Lett. 66, 52 (1995).

[86] S. Hofmann and J. Erlewein, Surf. Sci. 77, 591 (1978).

[87] J.J. Harris, D.E. Ashenford. C.T. Foxon. P.J. Dobson and B.A. Joyce. Appl. Phys. A 33. 87 (1984).

[88] H. Jorke. Surf. Sci. 193. 569 (1988).

[89] M.D. Williams. T.H. Chiu and F.G. Storz, J. Vac. Sci. Technol. B 13, 692 (1995)

[90] R. Arès. C.A. Tran and S.P. Watkins. Appl. Phys. Lett. 67. 15ï6 (1995).

[91] N. Grandjean. J. Massies and M. Leroux, Phys. Rev. B 53. 998 (1996).

[9:2] P. Disseix, J. Leymarie, A. Vasson, A. -M. Vasson, C. Monier, N. Grandjean, M. Leroux and J. Massies. Phys. Rev. B 55. 2406 (1997).

[93] K. Yamaguchi. T. Okada and F. Hiwatashi, Appl. Surf. Sci. 117/118. 700 (1997).

[94] W. Richter and D. Zahn. in Optical Characterization of Epitaxial Semiconductor Layers. G. Bauer, W. Richter (eds.). Springer. Berlin (1996).

[95] D.E. Aspnes, IEEE Journal of Quantum Electronics 25, 1056 (1989).

[96] J.D.E. McIntyre and D.E. Aspnes, Surf. Sci. 24. $41 i(19 i 1)$.

[97] I. Kamiya. D.E. Aspnes, H. Tanaka. L.T. Florez, J.P. Harbison and R. Bhat. Phys. Rev. Lett. 68, 62i (1992).

[98] I. Kamiya, D.E. Aspnes, L.T. Florez and J.P. Harbison. Phys. Rev. B 46, 15894 (1992).

[99] I. Kamiya, D.E. Aspnes, H. Tanaka, L.T. Florez. J.P. Harbison and R. Bhat, J. Vac. Sci. Technol. B 10, 1716 (1992). 
[100] D.E. Aspnes, R. Bhat. E. Colas, V.G. Keramidas. M.A. Koza and A.A. Studna, J. Vac. Sci. Technol. A 7, 711 (1989).

[101] D.E. Aspnes, I. Kamiya, H. Tanaka and R. Bhat. J. Vac. Sci. Technol. B 10. $1725(1992)$.

[102] D.W. Kisker, G.B. Stephenson, I. Kamiya, P.H. Fuoss. D.E. Aspnes, L. Mantese and S. Brennan. Phys. Stat. Sol. 152.9 (1995).

[103] I. Kamiya, L. Mantese. D.E. Aspnes. D.W. Kisker. P.H. Fuoss. G.B. Stephenson and S. Brennan. J. Cryst. Growth 163. 6 i (1996).

[104] S. Brennan, P.H. Fuoss, J.L. Kahn and D.W. Kisker, Nucl. Instr. Meth. Phys. Res. A 291. 86 (1990).

[105] S.M. Koch. O. Acher. F. Omnes. M. Defour. B. Drévillon and M. Razeghi, J. Appl. Phys. 69, 1389 (1991).

[106] M. Zorn. J. Jönsson. A. Krost. W. Richter. J.-Th. Zettler. K. Ploska and F. Reinhardt. J. ('ryst. (irouth 145, 5:3 (1994).

[10i] M. Zorn. J. Jönsson, W. Richter. J.-T. Zettler and K. Ploska. Phys. Stat. Sol. 152. 23 (1995).

[108] D.A. Harrison. Ph.D. Thesis, Department of Physics. Simon Fraser Lniversity (1998).

[109] C.F. Kilingshirn, Semiconductor Optics. Springer. Berlin (1995).

[110] J.M. Gerard and J.Y. Marzin. Appl. Phys. Lett. 53, 568 (1988).

[111] R. C'ingolani, O. Brandt, L. Tapfer, G. Scamarcio. G.C. La Rocca and K. Ploog. Phys. Rev. B, 42. 3209 (1990).

[112] O. Brandt. H. Lage and K. Ploog, Phys. Rev. B 43, 14285 (1991).

[113] S.S. Dosanjh, P. Dawson, M.R. Fahy, B.A. Joyce. R.A. Stradling and R. Murray, J. Cryst. Growth 127, 579 (1993). 
[114] M. Ilg and K.H. Ploog, Phys. Rev. B 48, 11512 (1993).

[115] M. Ilg. M.I. Alonso, A. Lehmann. K.H. Ploog and M. Hohenstein. J. Appl. Phys. 74. $i 188$ (1993).

[116] P.D. Wang. N.N. Ledentsov, C.M. Sotomayor Torres, P.S. Kop'ev and I.M. Ustinov. Appl. Phys. Lett. 64, 1526 (1994).

[117] P.D. Wang, N.N. Ledentsov, C.M. Sotomayor Torres. I.N. Yassievich. A. Pakhomov. A. Yu. Egonov. P.S. Kop'ev and V.M. Ustinov. Phys. Rev. B 50. 1604 (1994).

[118] S.S. Dosanjh. E.F. Bowser and J.J. Harris, Appl. Phys. Lett. 64. 2142 (1994).

[119] M.V. Belousov. N.N. Ledentsov. M.V. Maximov. P.D. Wang. I.N. Yasievich. N.N. Faleev. L.A. Kozin. V.M. Listinov, P.S. Kopev and (C.M. Sotomayor Torres. Phys. Rei. B 51, 143.46 (1995).

[120] M.I. Alonso. M. Ilg. K. Ploog and A. Trampert. J. Appl. Phys. 78. 1980 (1995).

[121] W. Li, Z. Wang, J. Liang, B. Xu. Z. Zhu. Z. Yuan and J. Li. Appl. Phys. Lett. 67. $1874(1995)$.

[122] R. Colombelli, J.M. Jancu. F. Beltram. L. Sorba. D. Bertho and C. Jouanin. Appl. Phys. Lett. 68, 1534 (1996).

[123] Z.L. Yuan. Z.Y. Xu, B.Z. Zheng, J.Z. Xu, S.S. Li. W. Ge. Y. Wang, J. Wang. L.L. Chang, P.D. Wang, C.M. Sotomayor Torres and N.N. Ledentsov, Phys. Rev. B 54, 16919 (1996).

[124] M.A. Tischler, N.G. Anderson and S.M. Bedair, Appl. Phys. Lett. 49. 1199 (1986).

[125] K. Taira, H. Kawai, I. Hase, K. Kaneko and N. Watanabe, Appl. Phys. Lett. 53, 495 (1988). 


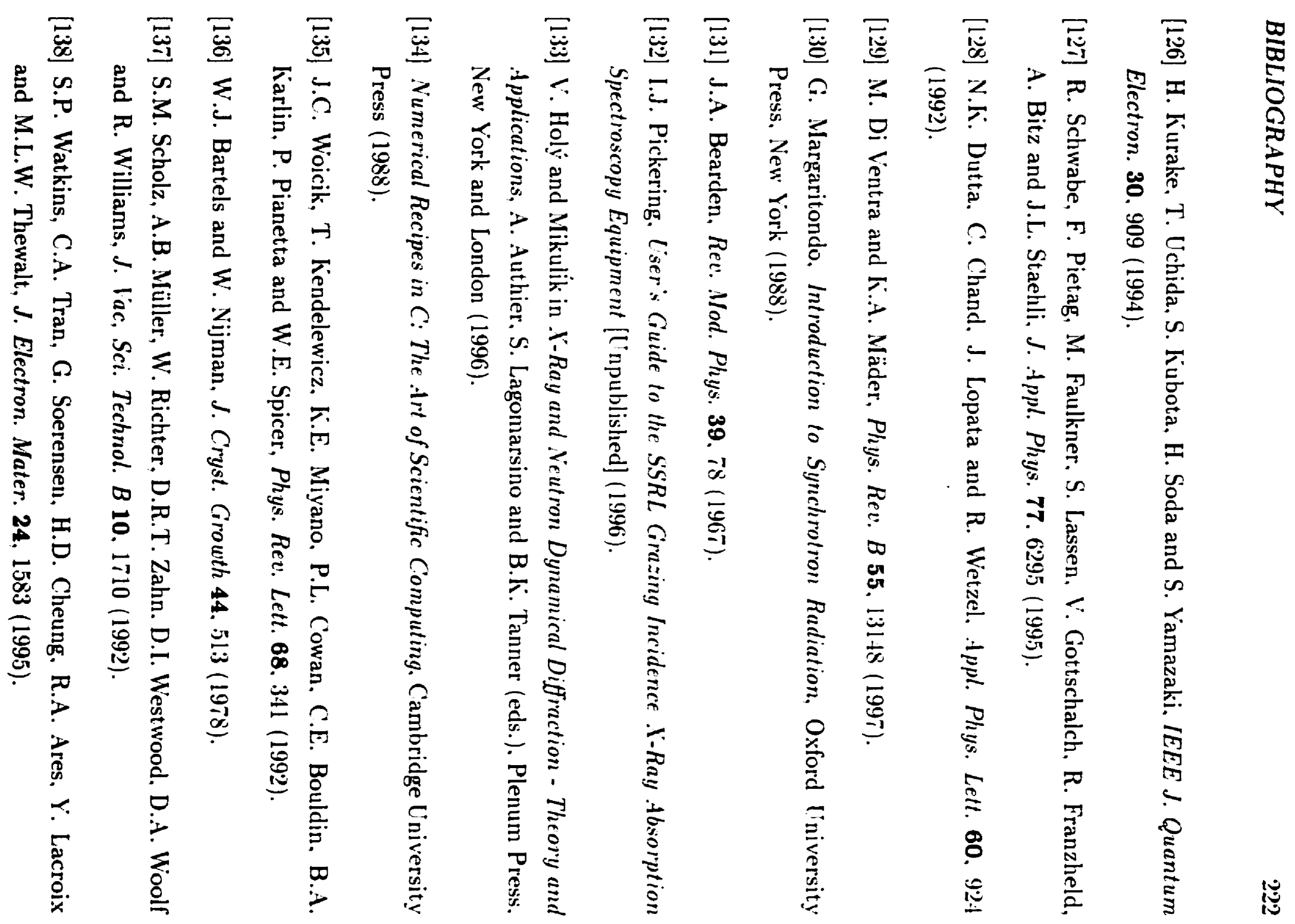


[139] M. Newville, P. Livins, Y. Yacoby, J.J. Rehr and E.A. Stern, Phys. Rev, B 47. 14126 (1993).

[140] G.G. Li, F. Bridges and C.H. Booth, Phys. Rev. B 52. 6332 (1995).

[141] A.L. Holmes Jr., M.E. Heimbuch. G. Fish. L.A. Coldren and S.P. Denbaars, J. Electron. Mater. 25. 965 (1996).

[142] J.A. Gupta, J.C. Woicik, S.P. Watkins, K.E. Miyano, J.G. Pellegrino and E.D. Crozier, J. C'ryst. Cirowth 195, 34 (1998).

[143] J.A. Gupta, S.P. Watkins, R. Arès and G. Soerensen. J. Cryst. Grouth 195. 205 (1998).

[144] J.A. Gupta. S.P. Watkins. E.D. Crozier. J.C. Woicik. D.A. Harrison. D.T. Jiang. I.J. Pickering and B.A. Karlin. [Submitted to Phys. Ree. B] (1999).

[145] A. Lessmann. S. Brennan. A. Munkholm. M. Schuster. H. Riechert and G. Materlik. Phys. Rev. B 59. 10801 (1999).

[146] International Tables for X-Ray Crystallography Vol. 4. Kynoch Press. Birmingham (1974).

[147] D.T. Cromer and D. Liberman, J. Chem. Phys. 53. 1891 (1970).

[148] S. Brennan and P.L. Cowan, Rev. Sci. Instr. 63. 8.50 (1992). 